\title{
INTERAÇÃO NÃO-LINEAR ENTRE ONDAS ATMOSFÉRICAS: UM POSSÍVEL MECANISMO PARA A CONEXÃO TRÓPICOS-EXTRATRÓPICOS EM BAIXA-FREQÜÊNCIA
}

Carlos Frederico Mendonça Raupp

TESE APRESENTADA AO INSTITUTO DE ASTRONOMIA, GEOFÍSICA E CIÊNCIAS ATMOSFÉRICAS

$$
\text { DA }
$$

UNIVERSIDADE DE SÃO PAULO

PARA A OBTENÇÃO DO TÍTULO DE

DOUTOR EM CIÊNCIAS

ÁREA: METEOROLOGIA

Orientador: Prof. Dr. Pedro Leite da Silva Dias

São Paulo, Março de 2006 


\section{AGRADECIMENTOS}

Ao Prof. Pedro Leite da Silva Dias: sua orientação sempre instigante e encorajadora, desde minha iniciação científica até o doutoramento, foi fundamental para a minha formação acadêmica. Meus agradecimentos e admiração pela sua competência e capacidade, bem como por seu entusiasmo e bom humor, sempre presentes e contagiantes.

Aos Professores Esteban Tabak (Courant Institute of Mathematical Sciences/New York University-EUA) e Paul Milewski (Department of Mathematics/University of WisconsinMadison-EUA), que tiveram uma participação fundamental no desenvolvimento deste trabalho, pois me orientaram conjuntamente ao longo da minha estadia de seis meses nestas duas instituições norte-americanas.

Aos professores do Departamento de Ciências Atmosféricas do IAG-USP, pela contribuição no processo de minha formação acadêmica, tanto na graduação quanto no mestrado e no doutorado.

Aos técnicos de informática e analistas de sistemas do Departamento de Ciências Atmosféricas do IAG-USP, pelo suporte durante o desenvolvimento desta pesquisa.

À Marisa, pelo constante e sempre atencioso apoio de secretaria durante o desenvolvimento deste trabalho e também pela amizade.

A todos os meus amigos e colegas do IAG-USP, pelo direto ou indireto auxílio durante o desenvolvimento deste trabalho e sobretudo por proporcionarem um ambiente sempre alegre e amigável.

Ao Conselho Nacional de Desenvolvimento Científico e Tecnológico (CNPQ), pelo suporte financeiro durante os quatro primeiros meses do meu doutoramento.

À Fundação de Amparo à Pesquisa do Estado de São Paulo (FAPESP), pelo apoio financeiro durante os 32 meses restantes do desenvolvimento desta pesquisa, tanto no Brasil quanto nos Estados Unidos. 
À Universidade de São Paulo, que concedeu recursos por meio de sua Pró-Reitoria de PósGraduação para a passagem aérea São Paulo/Nova Iorque/São Paulo, sem os quais seria impossível a realização do estágio de pesquisa no exterior, que por sua vez foi fundamental para o desenvolvimento deste trabalho bem como para minha formação científica. 
Aos meus pais, Elizabeth e Marco Antônio, pelo apoio incondicional e pelos ensinamentos e dedicação na minha educação. 


\section{SUMÁRIO}

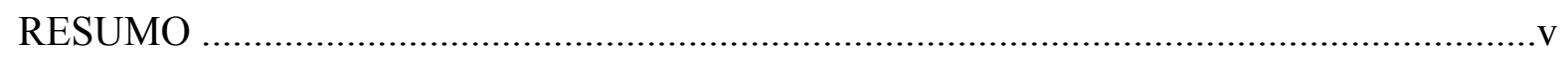

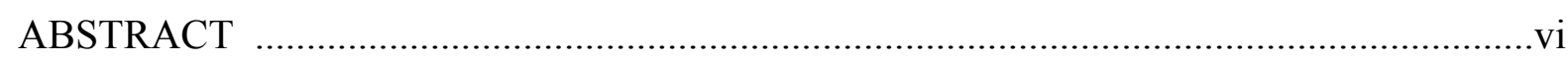

LISTA DE FIGURAS ............................................................................................. vii

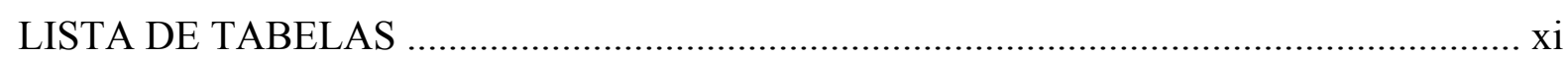

LISTA DOS PRINCIPAIS SÍMBOLOS …......................................................................

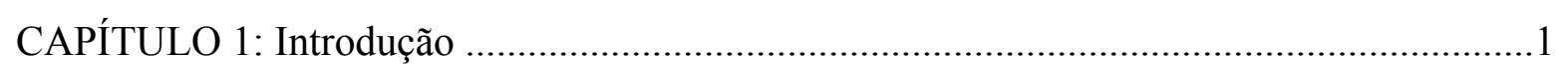

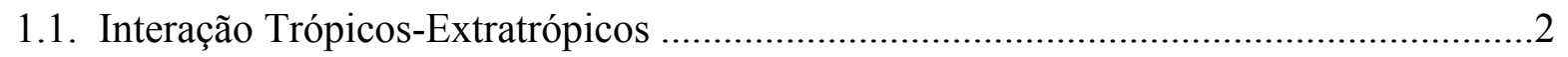

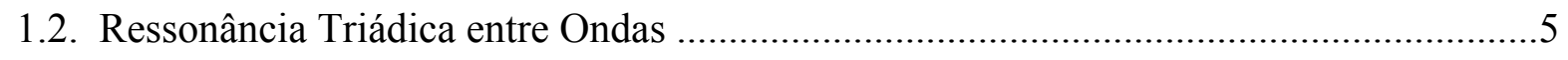

1.3. Objetivos Específicos do Trabalho e Organização do Texto ...........................................

CAPÍTULO 2: Equações Governantes e Método de Solução ...............................................11

2.1. Formulação do Modelo e Equações Básicas ......................................................................11

2.2. Equações Primitivas na Forma Adimensional e Aplicação do Método Assintótico de

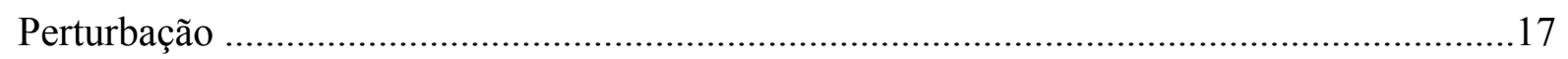

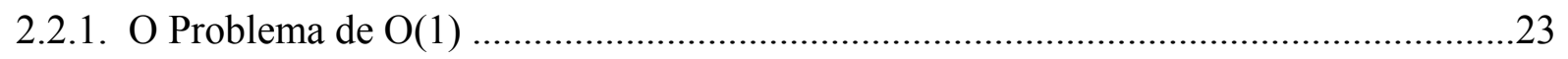

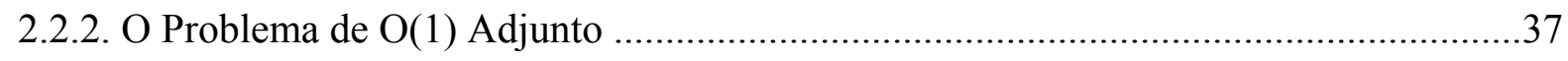

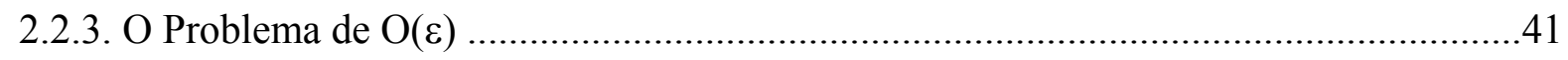

CAPÍTULO 3: Algumas Relações Energéticas Envolvendo as Ondas Equatoriais ...............58

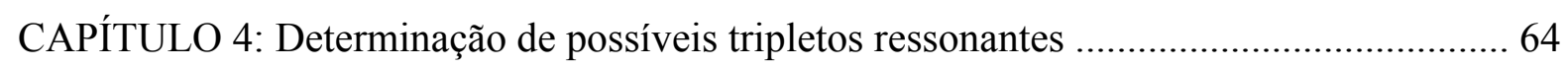

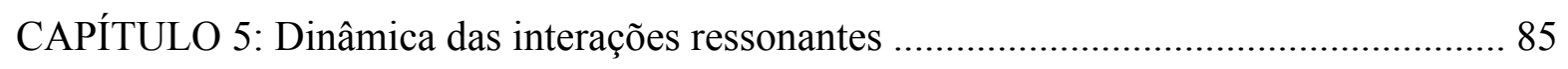

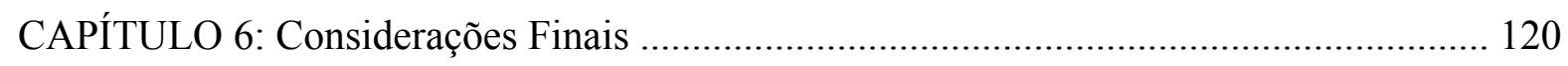

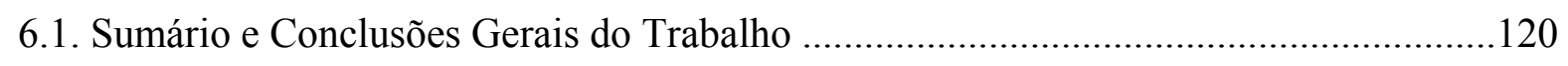

6.2. Sugestões para Pesquisas Futuras ....................................................................... 122

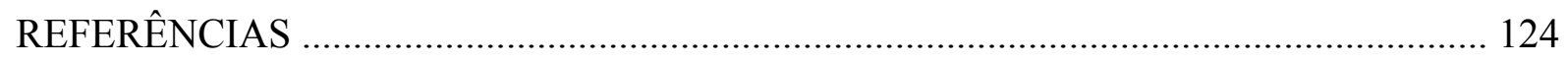

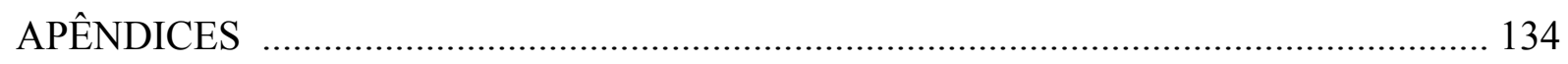

ANEXO: Artigo: Resonantly Interacting Equatorial Waves. Tellus 58A (2), 2006. (Aceito para Publicação). 


\section{RESUMO}

O método assintótico de múltiplas escalas temporais é usado neste trabalho para estudar as interações não lineares entre ondas na atmosfera no contexto das equações primitivas no plano $\beta$-equatorial em coordenadas isobáricas. As ondas equatoriais de Rossby, mista Rossby-gravidade, gravidade-inerciais e Kelvin, com diferentes estruturas verticais, são obtidas como soluções de ordem dominante. A partir da condição de solvabilidade do problema de ordem superior, foi obtido um modelo assintótico reduzido que governa a interação fracamente não linear entre as ondas numa determinada tríade ressonante. A conservação da energia total para a solução de ordem dominante do modelo de equações original implica que a soma dos coeficientes de interação numa tríade ressonante qualquer deve ser nula. Usando um método gráfico, foram encontradas algumas tríades ressonantes envolvendo ondas baroclínicas equatorialmente confinadas e ondas de Rossby barotrópicas possuindo significativa influência em médias e altas latitudes. Duas tríades ressonantes receberam especial atenção devido ao significativo acoplamento entre os modos e à possível relação desses modos com alguns aspectos observados acerca dos fenômenos de baixa freqüência na atmosfera. A primeira tríade é composta por uma onda de Rossby barotrópica possuindo o segundo modo meridional interagindo ressonantemente com uma onda mista de Rossby-gravidade com a estrutura vertical do primeiro modo baroclínico, ambas possuindo o mesmo número de onda zonal, por meio de um modo geostrófico zonalmente simétrico $(\mathrm{k}=$ 0 ) possuindo a mesma estrutura vertical da onda mista e o modo meridional $n=1$. A segunda tríade é constituída por uma onda mista de Rossby-gravidade com número de onda-2, uma onda de Kelvin com número de onda-1, ambas associadas ao primeiro modo baroclínico, e uma onda de Rossby barotrópica com número de onda-3 e modo meridional $\mathrm{n}=2$. A integração das equações reduzidas para essas duas tríades ressonantes mostra que para as amplitudes iniciais dos modos caracterizando magnitudes típicas de anomalias atmosféricas observadas, os modos na primeira tríade, em geral, trocam energia na escala intra-sazonal, enquanto os modos na segunda tríade trocam energia na escala intra-sazonal ou numa escala semi-anual, dependendo da amplitude inicial do modo de Rossby barotrópico. São discutidas as implicações dos resultados para a dinâmica da interação trópicos-extratrópicos na escala de baixa-freqüência do espectro dos movimentos atmosféricos. 


\begin{abstract}
The asymptotic method of multiple time-scales is used to investigate the nonlinear interactions among equatorial waves in an equatorial $\beta$-plane adiabatic primitive equation atmospheric model in isobaric coordinates. The equatorial Rossby, mixed Rossby-gravity, Kelvin and inertio-gravity waves, with several vertical structures, are obtained as the leadingorder solution. From the solvability condition of the problem, a reduced model governing the weakly nonlinear interaction of the waves in a particular resonant triad was obtained. The total energy conservation of the leading-order solution of the original model equations implies that the sum of the coupling coefficients in any resonant triad must be zero. Using a graphical approach, we determined some resonant triads involving equatorially trapped baroclinic waves and barotropic Rossby waves having large mid-latitude amplitude. Two particular interactions deserve special attention because of their strong coupling and their possible relation to observed features in the atmospheric circulation. One is characterized by a first baroclinic mode structure mixed Rossby-gravity wave interacting with a barotropic Rossby wave with the second gravest meridional mode, both having the same wavenumber, through a zonally symmetric geostrophic mode with the same vertical structure as the Yanai wave and having the $\mathrm{n}=1$ meridional mode. The other triad is composed of a zonal wavenumber-1 Kelvin wave, a zonal wavenumber-2 mixed Rossby-gravity wave, both with the first baroclinic mode vertical structure, and a barotropic zonal wavenumber-3 Rossby wave having the second gravest meridional mode. The barotropic Rossby waves in these two triad interactions have significant projection onto middle and higher latitudes. The integration of the triad equations for these particular interactions shows that, for the initial mode amplitudes characterizing typical magnitudes of atmospheric flow perturbations, the modes in the first triad usually exchange energy on intraseasonal time-scales, while the modes in the second triad exchange energy on either intraseasonal or semi-annual time-scale, depending on the initial condition. The implications of the results for the dynamics of tropics-extratropics interaction on low-frequency time-scale are discussed.
\end{abstract}




\section{LISTA DE FIGURAS}

Figura 2.1: Perfis de $\overline{\mathrm{T}}(\mathrm{p})$ (a) e $\bar{\rho}(\mathrm{p})$ (b) utilizados no presente trabalho para $\bar{\sigma}=2 \times 10^{-6}$ $\mathrm{m}^{4} \mathrm{~s}^{2} \mathrm{Kg}^{-2}$.

Figura 2.2: Estrutura das autofunções da equação da estrutura vertical (2.31) sujeita às condições de fronteira (2.33) para: (a) $\mathrm{m}=0$, (b) $\mathrm{m}=1$, (c) $\mathrm{m}=2$, (d) $\mathrm{m}=3$ e (e) $\mathrm{m}=4$.

Figura 4.1: Diagrama de dispersão das ondas de Rossby barotrópicas (ORB) com o modo meridional $\mathrm{n}=2$ e das seguintes ondas equatoriais associadas ao primeiro modo baroclínico $\mathrm{m}$ =1: ondas mistas de Rossby-gravidade (OMRG), ondas de Rossby (OR) para os modos meridionais $\mathrm{n}=1$ e $\mathrm{n}=2$, ondas de gravidade-inerciais com propagação para leste associadas ao modo meridional $\mathrm{n}=0$, ondas de gravidade-inerciais para oeste (OGO) e para leste (OGL) para os modos meridionais $\mathrm{n}=1$ e $\mathrm{n}=2$ e das ondas de Kelvin (OK). As curvas destacam a ressonância envolvendo as ondas de Rossby barotrópicas e as ondas mistas de Rossbygravidade por meio do modo geostrófico zonalmente simétrico com modo meridional $\mathrm{n}=1$.

Figura 4.2: Diagrama de dispersão das ondas de Rossby barotrópicas (ORB) com o modo meridional $\mathrm{n}=1$ e das seguintes ondas equatoriais para o primeiro modo baroclínico $\mathrm{m}=1$ : ondas mistas de Rossby-gravidade (OMRG), ondas de Rossby (OR) para os modos meridionais $n=1$ e $n=2$, ondas de gravidade-inerciais com propagação para leste associadas ao modo meridional $\mathrm{n}=0$, ondas de gravidade-inerciais para oeste (OGO) e para leste (OGL) com modos meridionais $n=1$ e $n=2$ e das ondas de Kelvin (OK). As curvas destacam a ressonância envolvendo a onda de Rossby barotrópica com $\mathrm{n}=1$ e número de onda $7(\mathrm{k} \approx$ $1,1)$ e duas ondas de Poincaré baroclínicas (modo vertical $\mathrm{m}=1$ ) e com modo meridional $\mathrm{n}=$ 1 , sendo uma com número de onda $1(\mathrm{k} \approx 0,157)$ e a outra com número de onda $8(\mathrm{k} \approx 1,256)$.

Figura 4.3: Diagrama de dispersão das ondas de Rossby barotrópicas (ORB) com o modo meridional $n=2$ e das seguintes ondas equatoriais para o segundo modo baroclínico $(m=2)$ : ondas mistas de Rossby-gravidade (OMRG), ondas de Rossby (OR) para os modos meridionais $\mathrm{n}=1$ e $\mathrm{n}=2$, ondas de gravidade-inerciais com propagação para leste (OGL) associadas ao modo meridional $\mathrm{n}=0$, ondas de gravidade-inerciais para oeste (OGO) e para leste (OGL) com modos meridionais $n=1$ e $n=2$ e das ondas de Kelvin (OK). As curvas 
destacam a ressonância envolvendo a onda de Rossby barotrópica com $n=2$ e número de onda $4(\mathrm{k} \approx 0,628)$ e duas ondas equatoriais baroclínicas (modo vertical $\mathrm{m}=2)$, sendo elas: uma onda mista de Rossby-gravidade com número de onda $1(\mathrm{k} \approx 0,157)$ e uma onda de gravidade-inercial para oeste com número de onda 5 e $\mathrm{n}=1$.

Figura 4.4: Diagrama de dispersão das ondas de Rossby barotrópicas (ORB) com o modo meridional $\mathrm{n}=2$ e das seguintes ondas equatoriais correspondentes ao primeiro modo baroclínico $\mathrm{m}=1$ : ondas mistas de Rossby-gravidade (OMRG), ondas de Rossby (OR) para os modos meridionais $n=1$ e $n=2$, ondas de gravidade-inerciais com propagação para leste associadas ao modo meridional $\mathrm{n}=0$, ondas de gravidade-inerciais para oeste (OGO) e para leste (OGL) com os modos meridionais $\mathrm{n}=1$ e $\mathrm{n}=2$ e das ondas de Kelvin (OK). As curvas destacam a ressonância envolvendo a onda de Rossby barotrópica com $n=2$ e número de onda $3(\mathrm{k} \approx 0,471)$ e duas ondas equatoriais baroclínicas (modo vertical $\mathrm{m}=1$ ), sendo estas: uma onda de Kelvin com número de onda zonal $1(\mathrm{k} \approx 0,157)$ e uma onda mista de Rossbygravidade com número de onda zonal $2(\mathrm{k} \approx 0,314)$.

Figura 5.1: Evolução temporal da energia modal para a tríade constituída por: uma onda de Rossby barotrópica com número de onda zonal $6(\mathrm{k} \approx 0,942)$ e modo meridional $\mathrm{n}=2$ (modo a), uma onda de Yanai com o mesmo número de onda zonal e referente ao primeiro modo baroclínico $(\mathrm{m}=1)$ (modo b) e um modo geostrófico zonalmente simétrico $(\mathrm{k}=0)$ com modo meridional $\mathrm{n}=1$ e a estrutura vertical correspondente ao modo baroclínico $\mathrm{m}=1$ (modo $\mathbf{c}$ ). A figura mostra a evolução temporal da energia das ondas para a condição inicial caracterizada por: (a) $\mathrm{R}_{\mathrm{b}}{ }^{0}=\mathrm{R}_{\mathrm{c}}{ }^{0}=2$ e (b) $\mathrm{R}_{\mathrm{b}}{ }^{0}=2$ e $\mathrm{R}_{\mathrm{c}}{ }^{0}=1$.

Figura 5.2: Seção latitude-pressão da componente meridional do vento ao longo da longitude de $45^{\circ} \mathrm{W}$ em: (a) $\mathrm{t}=0$; (b) $\mathrm{t}=14$ dias; (c) $\mathrm{t}=28$ dias e (d) $\mathrm{t}=41$ dias. A latitude (na abscissa) é apresentada em graus, enquanto a pressão (na ordenada) é apresentada em hPa. A componente meridional do vento corresponde à solução de ordem dominante do modelo de equações (2.1) truncada de modo a considerar somente as três ondas ressonantes constituintes da tríade 2 da Tabela 4.5. O campo de v é mostrado na figura acima na escala dimensional $(\mathrm{m} / \mathrm{s})$, usando a escala $(2.11)$ para $U=5 \mathrm{~m} / \mathrm{s}$. 
Figura 5.3: Distribuição horizontal dos campos do vento (vetor) e do geopotencial (contornos) em $\mathrm{p}=1000 \mathrm{hPa}$ associados à mesma solução das Figuras $5.1 \mathrm{~b}$ e $5.2 \mathrm{em}$ : (a) $\mathrm{t}=0$; (b) $\mathrm{t}=28$ dias. Os campos do vento horizontal e do geopotencial são dados, respectivamente, em $\mathrm{m} / \mathrm{s}$ e em $\mathrm{m}^{2} \mathrm{~s}^{-2}$, usando as escalas dadas por (2.11) para $\mathrm{U}=5 \mathrm{~m} / \mathrm{s}, \beta=2,3 \times 10^{-11} \mathrm{~m}^{-1} \mathrm{~s}^{-1} \mathrm{e} \mathrm{L}=$ $10^{6} \mathrm{~m}$.

Figura 5.4: Evolução temporal da divergência horizontal no nível de $200 \mathrm{hPa}$ em $\left(45^{\circ} \mathrm{W}, 12^{\circ} \mathrm{S}\right)$ (a) e da componente meridional do campo do vento no nível de $500 \mathrm{hPa}$ em $\left(45^{\circ} \mathrm{W}, 60^{\circ} \mathrm{S}\right)$ (b) associadas à solução referente às Figuras 5.1b, 5.2 e 5.3. Os campos de v e $\left(\frac{\partial u}{\partial x}+\frac{\partial \mathrm{v}}{\partial y}\right)$ são dados, respectivamente, em m/s e em $10^{-6} \mathrm{~s}^{-1}$, usando as escalas dadas por (2.11) para $\mathrm{U}=$ $5 \mathrm{~m} / \mathrm{s}, \beta=2,3 \times 10^{-11} \mathrm{~m}^{-1} \mathrm{~s}^{-1}$ e $\mathrm{L}=10^{6} \mathrm{~m}$.

Figura 5.5: Espectro de potência da componente meridional do vento no equador para o período de 30 dias durante o experimento ABLE 2-b, usando dados da reanálise do NCEPNCAR. O eixo da abscissa representa o comprimento de onda de $10.000 \mathrm{Km}$ a $1.000 \mathrm{Km}$ e o eixo das ordenadas refere-se ao período das ondas (em dias). A análise espectral foi realizada no setor longitudinal de $90^{\circ} \mathrm{W}$ a $0^{\circ}$. (Fonte: Santos et al. 2002)

Figura 5.6: Evolução temporal da energia modal para a tríade constituída por: uma onda mista de Rossby-gravidade com número de onda zonal $2(\mathrm{k} \approx 0,314)$ e com a estrutura vertical do primeiro modo baroclínico $\mathrm{m}=1$ (modo a), uma onda de Kelvin com número de onda zonal 1 $(\mathrm{k} \approx 0,157)$ e com a estrutura vertical associada ao primeiro modo baroclínico $\mathrm{m}=1$ (modo b) e uma onda de Rossby barotrópica com número de onda zonal $3(\mathrm{k} \approx 0,471)$ e com modo meridional $\mathrm{n}=2$ (modo $\mathbf{c})$. A figura mostra a evolução temporal da energia das ondas para a condição inicial caracterizada por: (a) $\mathrm{R}_{\mathrm{b}}{ }^{0}=5$ e $\mathrm{R}_{\mathrm{c}}{ }^{0}=0,2$; (b) $\mathrm{R}_{\mathrm{b}}{ }^{0}=5$ e $\mathrm{R}_{\mathrm{c}}{ }^{0}=0,5$ e (c) $\mathrm{R}_{\mathrm{b}}{ }^{0}=8$ e $\mathrm{R}_{\mathrm{c}}{ }^{0}=0,2$.

Figura 5.7: Distribuição horizontal dos campos do vento horizontal (vetor) e do geopotencial (contornos) em $\mathrm{p}=1000 \mathrm{hPa}$ associados à mesma solução mostrada na Figura 5.6a em: (a) $\mathrm{t}=$ 0 ; (b) $\mathrm{t}=25$ dias e (c) $\mathrm{t}=80$ dias. Os campos do vento horizontal e do geopotencial são dados, respectivamente, em $\mathrm{m} / \mathrm{s}$ e em $\mathrm{m}^{2} \mathrm{~s}^{-2}$, usando as escalas dadas por (2.11) para $\mathrm{U}=$ $5 \mathrm{~m} / \mathrm{s}, \beta=2,3 \times 10^{-11} \mathrm{~m}^{-1} \mathrm{~s}^{-1} \mathrm{e} \mathrm{L}=10^{6} \mathrm{~m}$. 
Figura 5.8: Análoga à Figura 5.7a, mas com as variáveis dispostas no nível $\mathrm{p}=500 \mathrm{hPa}$.

Figura 5.9: Evolução temporal da componente zonal do campo do vento no nível de 1000hPa em $\left(180^{\circ}, 0^{\circ}\right)$ (a); da componente zonal do campo do vento no nível de 500hPa em $\left(180^{\circ}, 25^{\circ} \mathrm{S}\right)$ (b) e da componente meridional do campo do vento no nível de $1000 \mathrm{hPa}$ em $\left(180^{\circ}, 0^{\circ}\right)(\mathrm{c})$. Os gráficos mostrados nessa figura correspondem à solução referente às Figuras 5.6a, 5.7 e 5.8. Os campos de u e v são dados em m/s, usando as escalas dadas por (2.11) para $\mathrm{U}=5 \mathrm{~m} / \mathrm{s}$.

Figura 5.10: Distribuição horizontal dos campos do vento horizontal (vetor) e do geopotencial (contornos) em $\mathrm{p}=1000 \mathrm{hPa}$ associados à mesma solução mostrada na Figura 5.6b no instante inicial $(\mathrm{t}=0)$. Os campos do vento horizontal e do geopotencial são dados, respectivamente, $\mathrm{em} \mathrm{m} / \mathrm{s} \mathrm{e} \mathrm{em} \mathrm{m}^{2} \mathrm{~s}^{-2}$, usando as escalas dadas por (2.11) para $\mathrm{U}=5 \mathrm{~m} / \mathrm{s}, \beta=2,3 \times 10^{-11} \mathrm{~m}^{-1} \mathrm{~s}^{-1} \mathrm{e}$ $\mathrm{L}=10^{6} \mathrm{~m}$. 


\section{LISTA DE TABELAS}

Tabela 2.1: Valores de $\lambda_{\mathrm{m}}, \mathrm{c}_{\mathrm{m}}$ e $\mathrm{c}_{\mathrm{m}} \beta \mathrm{L}^{2}\left(\operatorname{com} \beta=2,3 \times 10^{-11} \mathrm{~m}^{-1} \mathrm{~s}^{-1}\right.$ e $\left.\mathrm{L}=1000 \mathrm{Km}\right)$ para os primeiros 5 modos verticais, $\mathrm{m}=0,1,2,3 \mathrm{e} 4$, para $\mathrm{p}_{0}=1000 \mathrm{hPa}, \mathrm{p}_{\mathrm{T}}=100 \mathrm{hPa}, \bar{\sigma}=2 \times 10^{-6}$ $\mathrm{m}^{4} \mathrm{~s}^{2} \mathrm{Kg}^{-2}$ e os perfis de $\overline{\mathrm{T}}(\mathrm{p})$ e $\bar{\rho}(\mathrm{p})$ mostrados na Fig. 2.1.

Tabela 4.1: Valores de $\alpha_{\mathrm{m}}{ }^{\mathrm{j} 1}$ obtidos por (2.94) para: (a) $\mathrm{m}=0$, (b) $\mathrm{m}=1$, (c) $\mathrm{m}=2$, (d) $\mathrm{m}=3$ e (e) $\mathrm{m}=4$.

Tabela 4.2: Valores de $\theta_{m}^{j l}$ obtidos por (2.94) para: (a) $\mathrm{m}=0$, (b) $\mathrm{m}=1$, (c) $\mathrm{m}=2$, (d) $\mathrm{m}=3 \mathrm{e}$ (e) $\mathrm{m}=4$.

Tabela 4.3: Valores de $\vartheta_{m}^{j l}$ obtidos por (2.94) para: (a) $\mathrm{m}=0$, (b) $\mathrm{m}=1$, (c) $\mathrm{m}=2$, (d) $\mathrm{m}=3 \mathrm{e}$ (e) $\mathrm{m}=4$.

Tabela 4.4: Valores de $\varsigma_{m}^{j l}$ obtidos por (2.94) para: (a) $\mathrm{m}=0$, (b) $\mathrm{m}=1$, (c) $\mathrm{m}=2$, (d) $\mathrm{m}=3 \mathrm{e}$ (e) $\mathrm{m}=4$.

Tabela 4.5: Sumário das tríades ressonantes obtidas pelo método gráfico mostradas nas Figuras 4.1 a 4.4. A Tabela mostra, da esquerda para a direita, os modos constituintes das tríades e suas respectivas auto-freqüências e coeficientes de acoplamento. Os modos são caracterizados, da esquerda para a direita, pelo modo vertical, pelo número de onda zonal, pelo modo meridional e pelo tipo de onda. Os tipos de ondas mostrados são abreviados como segue: Rossby (R), mista Rossby-gravidade (M), Kelvin (K), gravidade-inerciais com propagação para oeste (GO) e ondas de gravidade-inerciais com propagação para leste (GL).

Tabela 4.6: Similar à Tabela 4.5, mas para os coeficientes de interação calculados com $\Lambda=0$ nas equações (2.92) e (2.83), ou seja, considerando como condições de fronteira na vertical $\mathrm{w}\left(\mathrm{x}, \mathrm{y}, \mathrm{p}_{0}, \mathrm{t}\right)=\mathrm{w}\left(\mathrm{x}, \mathrm{y}, \mathrm{p}_{\mathrm{T}}, \mathrm{t}\right)=0$. 


\section{LISTA DOS PRINCIPAIS SÍMBOLOS}

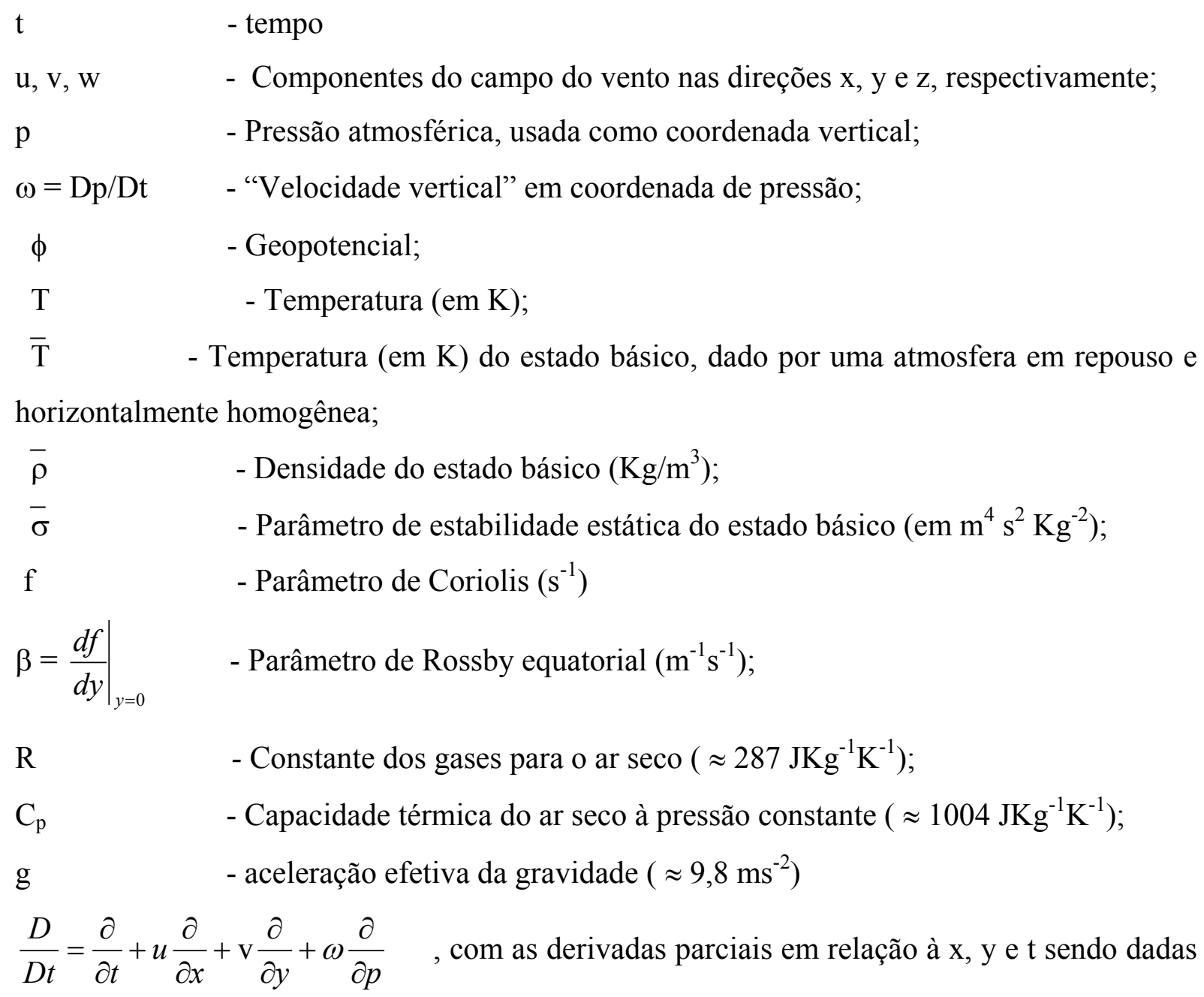
ao longo de superfícies isobáricas;

$\mathrm{p}_{0}$

- Pressão atmosférica referente à superfície isobárica próxima à superfície Terrestre;

$\mathrm{p}_{\mathrm{T}}$

- Pressão atmosférica referente à superfície isobárica próxima ao topo hipotético da troposfera;

$\mathrm{L}_{\mathrm{x}} \quad$ - Perímetro equatorial da Terra;

L - Escala de comprimento horizontal característica das perturbações;

U - Escala característica de $(\mathrm{u}, \mathrm{v})$;

$\Omega \quad$ - Escala característica de $\omega$;

$\widetilde{\widetilde{\sigma}} \quad$ - Parâmetro de estabilidade estática do estado básico na escala adimensional;

$\widetilde{\bar{\rho}} \quad$ - Densidade do estado básico na escala adimensional;

$\varepsilon$ - Parâmetro adimensional característico da amplitude das perturbações; 
$\mathrm{G}(\mathrm{p})$ ou $\mathrm{G}_{\mathrm{m}}(\mathrm{p})$

$\lambda_{\mathrm{m}}$

$\mathrm{c}, \mathrm{c}_{\mathrm{m}}$, ou $\mathrm{c}_{\mathrm{a}}$

- Escala longa de tempo $(=\varepsilon t)$

- Autofunções da estrutura vertical da solução do problema de $\mathrm{O}(1)$;

- Autovalores da equação da estrutura vertical do problema de $O(1)$;

- Constante de separação do problema de $\mathrm{O}(1)$;

$\varpi_{\mathrm{k}}, \varpi_{\mathrm{m}, \mathrm{k}, \mathrm{n}, \mathrm{r}}$ ou $\varpi_{\mathrm{a}} \quad$ - Autofreqüências dos modos normais da solução do problema de $\mathrm{O}(1)$;

k ou $\mathrm{k}_{\mathrm{a}} \quad$ - Número de onda zonal dos modos normais da solução do problema de $\mathrm{O}(1)$;

i - Unidade imaginária $(\mathrm{i}=\sqrt{-1})$;

$\mathrm{H}_{\mathrm{n}}(\mathrm{y}) \quad$ - Polinômio de Hermite de grau n;

$\xi_{\mathrm{a}}(\mathrm{y})=\left[\mathrm{u}_{\mathrm{a}}(\mathrm{y}), \mathrm{v}_{\mathrm{a}}(\mathrm{y}), \phi_{\mathrm{a}}(\mathrm{y})\right]^{\mathrm{T}} \quad$ - Autofunções da estrutura meridional da solução do problema de $\mathrm{O}(1)$;

$\mathrm{A}_{\mathrm{a}}(\tau)$

$\xi_{a}^{+}(y)$

$\widetilde{L}_{x}$

$\eta_{a}^{b c}$

$\alpha_{\mathrm{a}}^{\mathrm{bc}}, \mu_{\mathrm{a}}^{\mathrm{bc}}, \theta_{a}^{b c}, \vartheta_{a}^{b c}, \Psi_{a}^{b c} \mathrm{e} \varsigma_{a}^{b c}$
- Amplitude dos modos normais da solução do problema de $\mathrm{O}(1)$;

- Autofunções da estrutura meridional do problema de $\mathrm{O}(1)$ adjunto;

- Perímetro equatorial da Terra na escala adimensional;

estrutura vertical associadas aos modos a, b e c numa tríade ressonante;

$\mathrm{R}_{\mathrm{a}}(\tau)$

$\varphi_{\mathrm{a}}(\tau)$

$\Xi$

$\tilde{m}$
- Módulo da amplitude $A_{a}(\tau)$;

- Fase da amplitude $A_{a}(\tau)$;

- Argumento das funções elípticas de Jacobi;

- Parâmetro das funções elípticas de Jacobi; 


\section{CAPÍTULO 1}

\section{Introdução}

Com a extensão nos últimos anos da previsão numérica de tempo para médio (de 5 a 10 dias) e longo (> 10 dias) períodos, a interação entre as circulações atmosféricas tropical e de médias latitudes, assim como a dinâmica de fenômenos atmosféricos de baixa freqüência temporal, têm se tornado tópicos de suma importância prática. De fato, numa previsão numérica de longo período, torna-se notável a influência de fenômenos tropicais na previsão de tempo em regiões de latitudes médias e altas, assim como a ação dos fenômenos atmosféricos de baixa variabilidade como sendo os principais moduladores dos sistemas de escala sinótica.

A variabilidade de baixa freqüência é comumente definida na literatura especializada em Ciências Atmosféricas como sendo relacionada a fenômenos atmosféricos cujas freqüências são menores que a da escala sinótica (ou seja, períodos maiores que uma semana). Dos estudos observacionais acerca dos fenômenos atmosféricos de baixa freqüência emergem mapas mostrando estruturas ondulatórias, geograficamente dependentes, denominados padrões de teleconexões (ver, por exemplo, Wallace e Gutzler 1981 e Blackmon et al. 1984). Esses padrões de teleconexões atmosféricas são mais notáveis na média e na alta troposfera e são caracterizados por uma estrutura vertical equivalente barotrópica, ou seja, com os centros de ação apresentando a mesma fase ao longo de toda a troposfera. Alguns desses padrões apresentam centros de ação que se estendem dos trópicos até os extratrópicos, tais como os padrões do Pacífico/América do Norte (PNA) e do Pacífico/América do Sul (PSA), sendo, conseqüentemente, responsáveis pela influência de fenômenos tropicais como a Oscilação de Madden-Julian (OMJ) e o El Niño-Oscilação Sul (ENSO) sobre a dinâmica de médias e altas latitudes.

Assim, uma significativa parte da energia da atmosfera esta presente em modos equivalentes barotrópicos. Conseqüentemente, a maneira através da qual modos de variabilidade tropicais cuja estrutura vertical é essencialmente baroclínica, tais como a oscilação de Madden-Julian (OMJ), interagem com padrões barotrópicos equivalentes que apresentam uma significativa influência na circulação atmosférica de médias e altas latitudes constitui uma questão científica fundamental para a compreensão da dinâmica dos movimentos de baixa freqüência na atmosfera. $\mathrm{O}$ presente trabalho tem por objetivo explorar 
esta questão do ponto de vista teórico num contexto idealizado, analisando a dinâmica das interações não lineares fracas entre ondas baroclínicas equatorialmente confinadas e ondas de Rossby barotrópicas com uma significativa amplitude em latitudes médias e altas. Será apresentada a seguir uma breve revisão literária a respeito da conexão trópicos-extratrópicos, bem como da questão das interações não lineares entre ondas em fluidos geofísicos, em geral, e na atmosfera, em particular. Em seqüência, será destacada a importância do presente trabalho.

\subsection{Interação Trópicos-Extratrópicos}

As teleconexões trópicos-extratrópicos na região de baixa freqüência do espectro dos movimentos atmosféricos têm se tornado evidentes desde o estudo pioneiro de Horel e Wallace (1981) que, usando dados médios mensais para os períodos de inverno boreal de 1958 a 1978, verificaram a relação entre a variabilidade inter-anual das anomalias de temperatura da superfície do mar (TSM) sobre a região do Pacífico tropical e as anomalias de circulação atmosférica associadas ao padrão do PNA. Esta interação trópicos-extratrópicos também é evidente nos trabalhos de Mo e Livezey (1986), Lau e Boyle (1987) e Livezey e Mo (1987). Karoly (1989) desenvolveu um estudo similar ao de Horel e Wallace (1981), mas para o Hemisfério Sul, onde é destacada a relação entre as anomalias correspondentes ao fenômeno ENSO nos trópicos e as anomalias de circulação atmosférica relacionadas ao padrão do PSA. Na escala intra-sazonal, a relação entre as anomalias convectivas nos trópicos associadas à Oscilação de Madden-Julian (OMJ) e as anomalias da circulação atmosférica nos extratrópicos foi documentada por Liebmann e Hartmann (1984), Lau e Phillips (1986), Palmer (1988), Higgins e Mo (1997) e Mo e Higgins (1998). Recentemente, Carvalho et al. (2005) investigaram a relação entre a oscilação Antártica (AAO) e as anomalias de convecção e de circulação tropicais associadas aos fenômenos ENSO e OMJ durante o período de verão austral. Neste estudo, o padrão da oscilação Antártica foi definido como sendo a primeira função ortogonal empírica (EOF-1) obtida a partir de dados diários de anomalias de altura geopotencial dentro da faixa latitudinal de $20^{\circ} \mathrm{S}$ a $90^{\circ} \mathrm{S}$ no nível de $700 \mathrm{hPa}$ no período de 1979 a 2000 obtidos a partir dos dados da reanálise do NCEP-NCAR (National Center for Environmental Prediction-National Center for Atmospheric Research) (Kalnay et al. 1996). A partir de compostos de anomalias filtradas de TSM, vento zonal em 200hPa e de radiação de onda longa emergente (ROLE) durante as fases positiva e negativa da oscilação Antártica, eles sugerem que a convecção profunda na região do Pacífico tropical central, que está 
relacionada tanto ao ENSO quanto à OMJ, ou uma combinação de ambos fenômenos, modula a circulação do Hemisfério Sul de forma a favorecer as fases negativas da oscilação Antártica durante o período de verão do Hemisfério Sul.

Uma das possíveis idéias propostas para tentar explicar o mecanismo dinâmico responsável pela conexão trópicos-extratrópicos é baseada na teoria linear de propagação de ondas de Rossby barotrópicas a partir de uma fonte tropical de vorticidade. Esta teoria foi inicialmente proposta por Rossby (1945) e modificada posteriormente por Hoskins et al. (1977) e por Hoskins e Karoly (1981) e Hoskins e Ambrizzi (1993) com a inclusão dos efeitos da geometria esférica da Terra e de escoamentos básicos mais realistas. De acordo com esta teoria, os padrões de teleconexões trópicos-extratrópicos são resultantes da propagação da energia por ondas de Rossby barotrópicas forçadas por anomalias convectivas associadas a fenômenos tropicais como ENSO, OMJ e sistemas monçônicos. Neste contexto, vários estudos de modelagem numérica têm sido desenvolvidos usando modelos globais, com várias camadas na vertical, de equações primitivas linearizados em torno de estados básicos com cisalhamento tanto vertical quanto meridional (Webster 1972; 1981; 1982; Hoskins e Karoly 1981) com o intuito de analisar como forçantes térmicas tropicais estacionárias podem gerar uma resposta remota em médias e altas latitudes. É bastante claro a partir desses trabalhos que uma forçante térmica tropical pode gerar uma significativa resposta na circulação atmosférica, não somente nas vizinhanças da fonte mas também em regiões de médias e altas latitudes.

Uma análise detalhada da estrutura vertical da resposta atmosférica a fontes de calor tropicais revela que a resposta é essencialmente baroclínica nas vizinhanças da forçante e apresenta uma estrutura barotrópica equivalente nas regiões de médias e altas latitudes (Hoskins e Karoly 1981). Isto está em concordância com o estudo observacional de Silva Dias e Bonatti (1985) que, utilizando dados do FGGE-level IIIb (First Garp Global Experiment) no período de 29 de Janeiro a 16 de Fevereiro de 1979, projetaram os campos médios do vento e do geopotencial para este período nos modos verticais obtidos a partir de um modelo global de equações primitivas em coordenada sigma e mostram que o modo barotrópico equivalente é dominante nas latitudes mais altas da região analisada (América do Sul). À medida que se aproxima do equador, o modo interno caracterizado por uma única inversão de fase na troposfera ocorrendo na média troposfera passa a dominar a energética dos movimentos atmosféricos. Um ponto importante acerca dessa teoria é que o aquecimento diabático resultante da convecção profunda nos trópicos apresenta um perfil vertical típico que projeta a energia basicamente neste modo interno caracterizado por uma oposição de fase entre a alta e 
a baixa troposfera e com a inversão de fase ocorrendo exatamente na média troposfera (Yanai et al. 1973; Geisler e Stevens 1982; Lim e Chang 1983; DeMaria 1985; Fulton e Schubert 1985). De acordo com DeMaria (1985) e Lim e Chang (1983), o aquecimento diabático resultante da típica atividade convectiva tropical é bastante ineficiente em excitar o modo barotrópico. Com isso, Lim e Chang (1986), Kasahara e Silva Dias (1986) e Wang e Xie (1996) sugerem o cisalhamento vertical do campo básico, que constitui uma forma de acoplamento entre os modos verticais, como um possível mecanismo de excitação do modo externo através da transferência de energia dos modos internos preferencialmente excitados pelo aquecimento convectivo tropical. Kasahara e Silva Dias (1986) adotam um modelo espectral global de equações primitivas linearizadas em torno de um campo básico com cisalhamento vertical e meridional e obtêm a resposta estacionária a uma forçante especificada na equação da termodinâmica. O cálculo dos coeficientes de interação entre os modos verticais devida ao cisalhamento vertical do campo básico mostra que o modo externo é preferencialmente excitado por meio das auto-interações entre os modos internos. Kasahara e Silva Dias destacam a importância do cisalhamento vertical do campo básico para a geração de uma significativa resposta barotrópica em médias e altas latitudes a partir da forçante térmica tropical.

Uma outra hipótese acerca dos mecanismos que regem as teleconexões trópicosextratrópicos na escala temporal de baixa freqüência é baseada na teoria de instabilidade linear de escoamentos básicos tri-dimensionais proposta por Frederiksen (2002). Frederiksen (2002), utilizando um modelo de equações primitivas de duas camadas com a aproximação do plano $\beta$-equatorial linearizadas em torno de um campo básico dado pelo campo médio de Janeiro de 1979, mostra que um dos modos instáveis, rotulado como modo OMJ (Oscilação de Madden-Julian), é caracterizado pela estrutura espacial da oscilação de Madden-Julian nos trópicos para o potencial de velocidade, com a estrutura vertical do modo baroclínico caracterizado por uma única inversão de fase ao longo da troposfera, e uma estrutura barotrópica equivalente para a função de corrente nos extratrópicos, apresentando notáveis características dos padrões do PNA e da Euro-Ásia. Frederiksen utiliza nesse estudo uma parametrização do tipo Kuo generalizada com uma estabilidade estática úmida positiva em qualquer lugar do domínio, de tal forma que a instabilidade condicional de segunda espécie (wave-CISK) não é possível. Neste caso, de acordo com Frederiksen, o mecanismo de instabilidade para esse modo OMJ consiste numa instabilidade combinada na qual ambos os termos de conversão barotrópico e baroclínico estão presentes. O trabalho de Frederiksen (2002) constitui uma extensão tropical dos trabalhos acerca da instabilidade de escoamentos 
de base tridimensionais no contexto de médias latitudes desenvolvidos por Frederiksen (1982; 1983a, 1983b; 1983c).

Dessa forma, como pode ser verificado a partir da revisão literária apresentada nesta seção do presente capítulo, as teorias que têm sido desenvolvidas acerca da conexão trópicosextratrópicos na região de baixa freqüência do espectro dos movimentos atmosféricos são essencialmente baseadas nas equações linearizadas. Contudo, um outro mecanismo potencialmente importante para o acoplamento entre o modo externo e os modos internos e, conseqüentemente, para a conexão trópicos-latitudes médias é a interação não linear ondaonda. Entretanto, a dinâmica das interações não lineares onda-onda na atmosfera tem sido pouco explorada. As interações não lineares envolvendo ondas baroclínicas e equatorialmente confinadas e ondas barotrópicas com uma significativa projeção em médias latitudes devem constituir um mecanismo importante para a interação entre as circulações atmosféricas tropical e extratropical e precisam ser melhor compreendidas. Recentemente, Majda e Biello (2003), partindo das equações de Boussinesq para um modelo troposférico de duas camadas, com um campo básico possuindo cisalhamento vertical e meridional, desenvolveram um modelo assintótico reduzido para estudar a dinâmica de interações quase ressonantes entre ondas de Rossby equatoriais baroclínicas e ondas de Rossby barotrópicas com uma significativa projeção em médias latitudes. Majda e Biello fizeram um escalonamento do sistema original de tal forma que somente as ondas de Rossby ultra-longas, não dispersivas, representassem a solução de ordem dominante e, a partir da eliminação das soluções seculares (para maiores detalhes, ver o Capítulo 5 de Majda 2003), obtiveram um sistema de duas equações similares à equação de Korteweg-de Vries (equação K-dV), uma descrevendo a evolução da amplitude do pacote de ondas de Rossby barotrópicas e a outra descrevendo a evolução da amplitude do pacote de ondas equatoriais de Rossby baroclínicas. No modelo assintótico reduzido obtido por Majda e Biello, as duas equações similares às do tipo K-dV são acopladas uma a outra tanto por meio das interações onda-onda quanto por meio das interações das ondas com o campo básico com cisalhamento vertical.

\subsection{Ressonância Triádica entre Ondas}

No contexto dos movimentos ondulatórios na natureza, em geral, e em mecânica dos fluidos, em particular, existe uma clara distinção entre ondas dispersivas e não dispersivas. No contexto linear, esta distinção é claramente notável no sentido de que no caso de uma onda dispersiva cada componente harmônica na qual um distúrbio inicial qualquer é decomposto 
viaja com sua própria velocidade de grupo individual, enquanto numa onda não dispersiva todas as componentes harmônicas se propagam com a mesma velocidade de grupo, como um pacote, preservando a forma do distúrbio original. No entanto, à medida que a não linearidade aparece, esta distinção no comportamento de ondas dispersivas e não dispersivas torna-se ainda mais marcante, levando a mecanismos de interação entre os modos bastante distintos. Numa onda não dispersiva, uma vez que todos os modos viajam com a mesma velocidade, a interação entre eles é bastante significativa, produzindo uma mudança na forma do pacote de ondas e, como ocorre em algumas situações envolvendo ondas em fluidos, levando à quebra da onda e à formação de choque. Por outro lado, no caso de uma onda dispersiva, devido ao fato que cada modo se propaga com sua própria velocidade, levando a uma dispersão do distúrbio inicial, exceto num intervalo curto de tempo, a interação entre os modos neste caso é muito fraca. Porém, uma exceção a essa regra surge quando ocorre o fenômeno da ressonância. Neste caso, quando um conjunto de modos entra em ressonância de modo que a fase relativa entre eles não varia com o tempo, a interação entre esses modos torna-se expressiva. Por exemplo, num sistema dispersivo, com não linearidades quadráticas na mais baixa ordem, com a amplitude das ondas relativamente pequena (mas finita) de tal forma que estas preservem suas freqüências características dentro de um período típico de interação, quando a fase relativa entre o produto de duas ondas devido aos termos não lineares e uma terceira onda não varia no tempo, ou varia muito pouco, as ondas envolvidas nesse trio interagem significativamente. Neste caso, esse trio de ondas é comumente referido como tríade ressonante (ou tripleto ressonante). Assim, num sistema dispersivo contendo não linearidades quadráticas na mais baixa ordem, se a amplitude típica do sistema é pequena de tal forma que as ondas lineares constituam a solução dominante, somente os tripletos ressonantes contribuem significativamente para a evolução não linear do sistema.

Entretanto, em alguns problemas em mecânica de fluídos, como no caso de ondas de gravidade superficiais em águas profundas, a relação de dispersão das ondas é tal que encontrar tríades ressonantes torna-se um problema algébrico sem solução. Neste caso, tríades ressonantes entre ondas não são possíveis e, conseqüentemente, mesmo que o sistema contenha não linearidades quadráticas na mais baixa ordem, a não linearidade só se manifesta por meio dos termos cúbicos através da ressonância envolvendo quartetos de ondas (quatro ondas interagindo ressonantemente).

No caso do sistema de equações que rege a dinâmica da atmosfera, como será mostrado mais adiante, a existência de tríades ressonantes é possível. Além disso, neste sistema ambas ondas dispersivas e não dispersivas coexistem. Neste caso, como será 
mostrado no decorrer do presente trabalho, é possível inclusive que uma das componentes de uma onda de Kelvin (não dispersiva) interaja significativamente com duas ondas dispersivas por meio de uma ressonância triádica.

A dinâmica reduzida de um único tripleto ressonante é usualmente descrita por um sistema Hamiltoniano de três equações diferenciais ordinárias acopladas que governam a evolução temporal da amplitude das ondas. Este sistema comumente possui, além da Hamiltoniana, um número suficiente de integrais primeiras de forma a ser completamente integrável em termos das funções elípticas de Jacobi.

O fenômeno da ressonância não linear entre ondas foi inicialmente investigado por Phillips (1960), que analisou o papel dos termos não lineares na evolução das ondas de gravidade superficiais de amplitude finita utilizando um método de perturbação assintótico. Phillips (1960) destaca que interações triádicas não são possíveis no espectro das ondas de gravidade de superfície, e a não linearidade neste caso se manifesta na mais baixa ordem por meio de quartetos de ondas, através dos termos cúbicos. Entretanto, McGoldrick (1965) mostra que ressonâncias triádicas por meio de não linearidades quadráticas são possíveis no contexto de ondas de gravidade-capilares. Uma rica e completa discussão sobre ressonância triádica entre ondas dispersivas é encontrada em Bretherton (1964), que analisa um problema de uma simples equação de onda "forçada" por um termo quadrático. No contexto da dinâmica de fluidos geofísicos em rotação, o fenômeno da ressonância triádica entre ondas foi talvez primeiramente estudado por Longuet-Higgins e Gill (1967), que analisaram as interações não lineares ressonantes envolvendo ondas de Rossby num modelo barotrópico e não divergente. Duffy (1974) estudou as interações ressonantes no modelo de água-rasa com a aproximação do plano- $\beta$ de médias latitudes. Neste cenário, as interações triádicas são caracterizadas por duas ondas de gravidade-inerciais interagindo por meio de uma onda de Rossby, não sendo algebricamente possível a existência de tríades ressonantes envolvendo somente ondas de gravidade-inerciais. Domaracki e Loesch (1977) e Loesch e Deininger (1979) estenderam a análise de Duffy (1974) para o plano $\beta$-equatorial, analisando as interações triádicas envolvendo as ondas equatoriais utilizando o método de perturbação assintótico baseado em múltiplas escalas. Ripa (1981) formulou o problema da interação triádica onda-onda usando o formalismo de Galerkin com as funções base dadas pelas próprias autofunções do problema linear. Ripa aplicou tal formalismo tanto às ondas de Rossby barotrópicas não divergentes quanto às ondas de gravidade internas se propagando 
num plano vertical. Em seguida, Ripa (1983a,b) estendeu tal formalismo para o guia de onda equatorial, analisando as interações triádicas nas equações da água-rasa no plano $\beta$-equatorial.

Recentemente, Raupp e Silva Dias (2006) resolveram as equações da água-rasa não lineares no plano $\beta$-equatorial utilizando o método espectral com a base definida pelas autofunções das equações linearizadas e, aplicando o formalismo de Ripa (1983a), estudaram as interações triádicas ressonantes envolvendo ondas equatoriais. Raupp e Silva Dias (2006) (artigo em anexo) analisaram a dinâmica de um tripleto ressonante e a dinâmica de dois tripletos acoplados por meio de um único modo. Raupp e Silva Dias destacam a importância das ondas de gravidade-inerciais para a troca de energia entre dois tripletos diferentes. Um outro aspecto importante destacado por Raupp e Silva Dias (2006) é que as interações triádicas envolvendo as ondas equatoriais podem ter possíveis implicações para a variabilidade de baixa freqüência dos movimentos atmosféricos (variabilidade intra-sazonal e de período ainda mais longo), uma vez que os modos tipicamente trocam energia na escala intra-sazonal e/ou numa escala ainda mais longa, dependendo da amplitude inicial dos modos.

Dessa forma, os resultados apresentados por Raupp e Silva Dias (2006) sugerem que as implicações das interações triádicas ressonantes para a variabilidade de baixa freqüência dos movimentos atmosféricos podem ser ainda mais evidentes num modelo atmosférico mais realista. Além disso, Krishnamurti e Chakraborty (2005) desenvolveram um estudo observacional que reforça a importância das interações triádicas ressonantes na atmosfera. Krishnamurti e Chakraborty utilizam um conjunto de dados obtidos a partir das reanálises do ECMWF (European Center for Medium Range Weather Forecasting) durante o ano de 2001 e, através de uma análise de Fourier, mostram que durante os períodos de intensa atividade da OMJ ocorre sempre uma notável transferência de energia cinética de modos com freqüência na escala sinótica para modos com freqüência na escala de 30-60 dias. Um ponto importante verificado por Krishnamurti e Chakraborty é que essas interações são sempre triádicas e que nesses períodos de intensa transferência de energia cinética as freqüências dos três modos sempre satisfazem à condição de ressonância triádica, rotulada pelos autores como "phase locking”. Krishnamurti e Chakraborty também calculam os fluxos na camada limite planetária no espaço de Fourier, que são representados por produtos triplos entre modos, e destacam a importância da condição de ressonância entre as freqüências dos três modos ("phase locking") para a intensidade desses fluxos. Logo, o estudo de Krishnamurti e Chakraborty (2005) é bastante importante e reforça a necessidade de se explorar mais a fundo a dinâmica das interações triádicas ressonantes na atmosfera. 
Dessa forma, a dinâmica das interações triádicas ressonantes na atmosfera será investigada neste trabalho no contexto da versão adiabática das equações primitivas no plano $\beta$-equatorial em coordenadas isobáricas. A dinâmica reduzida para um único tripleto ressonante é obtida a partir do sistema de equações primitivas original através da aplicação da teoria clássica de perturbação assintótica baseada no método de múltiplas escalas temporais.

\subsection{Objetivos Específicos do Trabalho e Organização do Texto}

O presente trabalho tem por objetivo estudar a dinâmica das interações não lineares entre ondas na atmosfera e as possíveis implicações dessas interações para a conexão trópicos-extratrópicos e para a variabilidade de baixa freqüência dos movimentos atmosféricos. As equações dinâmicas não lineares adotadas no presente trabalho para tal propósito são dadas pelas equações primitivas no plano $\beta$-equatorial. $\mathrm{O}$ foco no presente estudo será dado aos outros tipos de ressonância não explorados por Majda e Biello (2003). De fato, como discutido na seção anterior deste capítulo, qualquer sistema dispersivo possuindo não linearidades quadráticas na mais baixa ordem pode potencialmente exibir o fenômeno da ressonância triádica entre ondas como representativo do efeito não linear de ordem dominante. Como será mostrado mais adiante neste trabalho, a relação de dispersão das ondas lineares obtidas como solução de ordem dominante do modelo aqui adotado permite a existência de ressonâncias triádicas entre as ondas. Além disso, embora a dinâmica de tripletos ressonantes tenha sido extensivamente estudada numa diversa gama de problemas físicos, como destacado na seção anterior, esta dinâmica, até onde se tem conhecimento, não foi ainda explorada no contexto das equações primitivas que governam a dinâmica dos movimentos de grande-escala da atmosfera. Logo, o presente trabalho objetiva estudar as possíveis interações triádicas ressonantes entre ondas no modelo atmosférico governado pelas equações primitivas no plano $\beta$-equatorial, num contexto idealizado, e explorar as possíveis implicações dessas interações para a dinâmica das teleconexões trópicos-extratrópicos, bem como para a variabilidade de baixa freqüência dos movimentos atmosféricos. Uma vez que o foco do presente estudo será dado às interações triádicas ressonantes entre ondas devidas somente aos termos não lineares nas equações governantes, alguns processos físicos importantes na atmosfera, tais como os efeitos de aquecimento/resfriamento diabático e de dissipação, não serão considerados no presente trabalho com o intuito de simplificar a análise matemática. 
O restante do presente trabalho é organizado como segue. No Capítulo 2 a teoria clássica de perturbação assintótica baseada em múltiplas escalas temporais é aplicada às equações governantes para reduzir o sistema original de equações diferenciais parciais a um sistema de equações reduzidas que descrevem a evolução fracamente não linear da amplitude das ondas numa determinada tríade ressonante. No Capítulo 3 são obtidas relações energéticas gerais para as ondas obtidas como solução de ordem dominante do sistema original com o intuito de obter alguns vínculos energéticos que as ondas numa dada tríade ressonante devem satisfazer. No Capítulo 4 algumas tríades ressonantes envolvendo duas ondas equatoriais baroclínicas associadas ao mesmo modo interno e uma onda de Rossby barotrópica são encontradas a partir das curvas de dispersão das ondas através de um método gráfico. $\mathrm{O}$ Capítulo 5 é destinado à analisar a dinâmica dessas interações ressonantes através da integração das equações reduzidas para uma tríade ressonante e à explorar às possíveis implicações das trocas de energia entre os modos envolvidos nessas tríades para a conexão trópicos-extratrópicos e para a variabilidade de baixa freqüência dos movimentos atmosféricos. No Capítulo 6 são apresentadas as conclusões gerais do trabalho e algumas sugestões para pesquisa futura. 


\section{CAPÍTULO 2}

\section{Equações Governantes e Método de Solução}

\subsection{Formulação do Modelo e Equações Básicas}

Uma vez que o presente trabalho tem por objetivo estudar as interações não lineares entre as ondas de amplitude finita na atmosfera, visando abordar as implicações acerca da dinâmica da interação entre as circulações atmosféricas tropical e extratropical, considera-se no presente trabalho um modelo que descreve a evolução das perturbações de grande-escala dos movimentos atmosféricos secos em relação a um estado básico caracterizado por uma atmosfera em repouso, horizontalmente homogênea e estavelmente estratificada na vertical. Os efeitos dinâmicos da rotação da Terra e da esfericidade da mesma sobre os movimentos de grande-escala são representados pela aproximação do plano $\beta$-equatorial, na qual o parâmetro de Coriolis é dado por $\mathrm{f}=\beta \mathrm{y}$, sendo y a distância com relação ao equador orientada para norte e $\beta=\left.\frac{d f}{d y}\right|_{y=0}$ é o parâmetro de Rossby, que neste caso é suposto constante. O modelo em questão é representado pela seguinte versão adiabática das equações primitivas usando a pressão atmosférica p como coordenada vertical:

$\frac{\partial u}{\partial t}+u \frac{\partial u}{\partial x}+\mathrm{v} \frac{\partial u}{\partial y}+\omega \frac{\partial u}{\partial p}-\beta y \mathrm{v}+\frac{\partial \phi}{\partial x}=0$

$\frac{\partial \mathrm{v}}{\partial t}+u \frac{\partial \mathrm{v}}{\partial x}+\mathrm{v} \frac{\partial \mathrm{v}}{\partial y}+\omega \frac{\partial \mathrm{v}}{\partial p}+\beta y \mathrm{u}+\frac{\partial \phi}{\partial y}=0$

$\frac{\partial \phi}{\partial p}=-\frac{R T}{p}$

$\frac{\partial \mathrm{u}}{\partial x}+\frac{\partial \mathrm{v}}{\partial y}+\frac{\partial \omega}{\partial p}=0$

$\frac{\partial \mathrm{T}}{\partial t}+u \frac{\partial \mathrm{T}}{\partial x}+\mathrm{v} \frac{\partial \mathrm{T}}{\partial y}+\omega \frac{\partial \mathrm{T}}{\partial p}-\omega \frac{R T}{p C_{p}}-\bar{S}_{p} \omega=0$

onde $\mathrm{u}$ e v correspondem às perturbações das componentes do campo do vento nas direções $\mathrm{x}$ (para leste) e y (para norte), respectivamente; $\omega=\mathrm{Dp} / \mathrm{Dt}$ refere-se à perturbação da 
velocidade vertical em coordenada-p e $\phi$ à perturbação do campo do geopotencial. T é a perturbação do campo da temperatura (em K) e todas as derivadas parciais com relação a x, y e $\mathrm{t}$ nas equações acima se dão ao longo de superfícies isobáricas. $\overline{\mathrm{S}}_{\mathrm{p}}$ corresponde ao parâmetro de estabilidade estática do estado básico, dado por

$$
\bar{S}_{p}=R \frac{\bar{T}}{p C_{p}}-\frac{d \bar{T}}{d p}
$$

onde $\overline{\mathrm{T}}(\mathrm{p})$ é a temperatura referente ao estado básico. Para uma atmosfera básica com estratificação estável, tem-se que $\overline{\mathrm{S}}_{\mathrm{p}}>0$. Nas equações (2.1) e (2.2) acima, $\mathrm{C}_{\mathrm{p}}$ representa a capacidade térmica do ar seco a pressão constante e $\mathrm{R}$ refere-se à constante dos gases para $\mathrm{o}$ ar seco.

Substituindo a condição de equilíbrio hidrostático (2.1c) em (2.1e), pode-se reescrever a equação da termodinâmica da seguinte forma:

$\frac{\partial}{\partial t} \frac{\partial \phi}{\partial p}+u \frac{\partial}{\partial x} \frac{\partial \phi}{\partial p}+\mathrm{v} \frac{\partial}{\partial y} \frac{\partial \phi}{\partial p}+\omega \frac{\partial^{2} \phi}{\partial p^{2}}+\frac{\omega}{p} \frac{\partial \phi}{\partial p}(1-\kappa)+\bar{\sigma} \omega=0$

onde $\kappa=\mathrm{R} / \mathrm{C}_{\mathrm{p}}$ e $\bar{\sigma}=\overline{\mathrm{S}}_{\mathrm{p}} \mathrm{R} / \mathrm{p}$. Logo, o parâmetro de estabilidade estática $\bar{\sigma}$ também é positivo para uma atmosfera básica com estratificação estável. Como condições de fronteira na vertical supõe-se que a perturbação da velocidade vertical real do vento, representada por $\mathrm{w}=1 / \mathrm{g} \mathrm{D} \phi / \mathrm{Dt}$, sendo g a aceleração efetiva da gravidade, seja nula na superfície $(\mathrm{z}=0)$ e num determinado topo de altura finita $\mathrm{z}=\mathrm{z}_{\mathrm{T}}$ da troposfera. Entretanto, surge uma dificuldade adicional ao adotar tais condições de fronteira no sistema de coordenadas isobáricas, uma vez que a pressão atmosférica não é constante ao longo da superfície $\mathrm{z}=0$ e do topo $\mathrm{z}=\mathrm{z}_{\mathrm{T}}$ num estado perturbado qualquer do modelo. A superfície e o topo hipotético aqui considerado somente representam superfícies isobáricas na ausência de perturbações, ou seja, quando o estado da atmosfera coincide com o estado básico considerado. Entretanto, como o presente estudo tem como foco ondas de amplitude pequena (ou fracamente não lineares), é razoável 
supor que ambos a superfície terrestre e o topo hipotético da atmosfera aqui considerado estejam próximos às superfícies isobáricas $\mathrm{p}=\mathrm{p}_{0} \mathrm{e} \mathrm{p}=\mathrm{p}_{\mathrm{T}}$, respectivamente. Neste caso, o geopotencial e a pressão na superfície e no topo da atmosfera podem ser relacionados através das seguintes expansões em séries de Taylor:

$$
\begin{aligned}
& \phi_{t o t}(z=0)=0=\bar{\phi}\left(p_{0}\right)+\phi\left(x, y, p_{0}, t\right)+\left.\frac{d \bar{\phi}}{d p}\right|_{p=p_{0}}\left(p(z=0)-p_{0}\right)+T O S \\
& \phi_{t o t}\left(z=z_{T}\right)=\bar{\phi}\left(p_{T}\right)+\phi\left(x, y, p_{T}, t\right)+\left.\frac{d \bar{\phi}}{d p}\right|_{p=p_{T}}\left(p_{T}-p\left(z=z_{T}\right)\right)+T O S
\end{aligned}
$$

onde TOS em (2.4) representam termos de ordem superior à primeira ordem, incluindo inclusive os termos $\left.\frac{\partial \phi}{\partial p}\right|_{p=p_{0}}\left(p(z=0)-p_{0}\right) \mathrm{e}^{\left.\frac{\partial \phi}{\partial p}\right|_{p=p_{T}}\left(p_{T}-p\left(z=z_{T}\right)\right)}$. Esses termos são incluídos em TOS em (2.4) pois, como será mostrado em seguida, eles resultam em termos não lineares cúbicos na expansão das condições de fronteira verticais. $\mathrm{Na}$ equação (2.4) acima, $\mathrm{p}(\mathrm{z}=0)=\mathrm{p}(\mathrm{x}, \mathrm{y}, \mathrm{z}=0, \mathrm{t}) \quad \mathrm{e} \mathrm{p}\left(\mathrm{z}=\mathrm{z}_{\mathrm{T}}\right)=\mathrm{p}\left(\mathrm{x}, \mathrm{y}, \mathrm{z}=\mathrm{z}_{\mathrm{T}}, \mathrm{t}\right) \quad$ representam as pressões atmosféricas ao longo da superfície e do topo, respectivamente, enquanto $\mathrm{p}_{0}$ e $\mathrm{p}_{\mathrm{T}}$ correspondem aos valores constantes representativos das superfícies isobáricas próximas a estes e $\phi_{\text {tot }}$ denota o geopotencial total, ou seja, a soma do geopotencial dos estados básicos e perturbados $\left(\phi_{\text {tot }}=\bar{\phi}+\phi\right)$. Uma vez que $\phi_{\text {tot }}(\mathrm{z}=0)=\bar{\phi}\left(\mathrm{p}_{0}\right)=0$ e $\phi_{\text {tot }}\left(\mathrm{z}=\mathrm{z}_{\mathrm{T}}\right)=\bar{\phi}\left(\mathrm{p}_{\mathrm{T}}\right)$, segue que

$$
\begin{aligned}
& p(x, y, z=0, t)=p_{0}+\bar{\rho}\left(p_{0}\right) \phi\left(x, y, p_{0}, t\right)+O\left(\phi^{2}\right) \\
& p\left(x, y, z=z_{T}, t\right)=p_{T}-\bar{\rho}\left(p_{T}\right) \phi\left(x, y, p_{T}, t\right)+O\left(\phi^{2}\right)
\end{aligned}
$$

sendo $\bar{\rho}=\bar{\rho}(\mathrm{p})$ a densidade do estado básico. Na equação (2.5) acima utilizou-se a suposição de que o estado básico satisfaz à condição do equilíbrio hidrostático e à equação do estado para um gás ideal, ou seja, 


$$
\frac{d \bar{\phi}}{d p}=-\frac{1}{\bar{\rho}}
$$

Assim, desprezando os termos de ordem quadrática nas expansões $(2.5 \mathrm{a}, \mathrm{b})$ e substituindo as expressões resultantes nas condições de fronteira do modelo, tem-se que:

$$
\begin{aligned}
& w(x, y, p(z=0), t)=w\left(x, y, p_{0}+\bar{\rho}_{0} \phi\left(x, y, p_{0}, t\right), t\right)=0 \\
& w\left(x, y, p\left(z=z_{T}\right), t\right)=w\left(x, y, p_{T}-\bar{\rho}_{T} \phi\left(x, y, p_{T}, t\right), t\right)=0
\end{aligned}
$$

onde $\bar{\rho}_{0}=\bar{\rho}\left(\mathrm{p}_{0}\right)$ e $\bar{\rho}_{\mathrm{T}}=\bar{\rho}\left(\mathrm{p}_{\mathrm{T}}\right)$.

Fazendo uma expansão em série de Taylor em $(2.7 \mathrm{a}, \mathrm{b})$ em torno de $\mathrm{p}=\mathrm{p}_{0}$ e $\mathrm{p}=\mathrm{p}_{\mathrm{T}}$, segue que as condições de fronteira na vertical podem ser expressas da seguinte forma:

$$
\begin{aligned}
& w\left(x, y, p_{0}, t\right)+\left.\frac{\partial w}{\partial p}\right|_{p=p_{0}} \bar{\rho}_{0} \phi\left(x, y, p_{0}, t\right)+O\left(\phi^{2}\right)=0 \\
& w\left(x, y, p_{T}, t\right)-\left.\frac{\partial w}{\partial p}\right|_{p=p_{T}} \bar{\rho}_{T} \phi\left(x, y, p_{T}, t\right)+O\left(\phi^{2}\right)=0
\end{aligned}
$$

Dado que $\mathrm{w}=\frac{1}{g} \frac{D \phi}{D t}=\frac{1}{g}\left(\frac{\partial \phi}{\partial t}+u \frac{\partial \phi}{\partial x}+\mathrm{v} \frac{\partial \phi}{\partial y}+\omega \frac{\partial \phi}{\partial p}+\omega \frac{d \bar{\phi}}{d p}\right)$, onde as derivadas parciais com relação a t, x e y se dão ao longo de superfícies isobáricas, pode-se reescrever as condições de fronteira na vertical dadas por (2.8) da seguinte forma:

$$
\begin{aligned}
& \frac{\partial \phi}{\partial t}+u \frac{\partial \phi}{\partial x}+\mathrm{v} \frac{\partial \phi}{\partial y}+\omega \frac{\partial \phi}{\partial p}-\frac{\omega}{\bar{\rho}_{0}}+\bar{\rho}_{0} \phi\left(x, y, p_{0}, t\right) \frac{\partial}{\partial p}\left(\frac{\partial \phi}{\partial t}+u \frac{\partial \phi}{\partial x}+\mathrm{v} \frac{\partial \phi}{\partial y}+\omega \frac{\partial \phi}{\partial p}-\frac{\omega}{\bar{\rho}}\right)=0 \\
& \mathrm{em} \mathrm{p}=\mathrm{p}_{0}
\end{aligned}
$$




$$
\frac{\partial \phi}{\partial t}+u \frac{\partial \phi}{\partial x}+\mathrm{v} \frac{\partial \phi}{\partial y}+\omega \frac{\partial \phi}{\partial p}-\frac{\omega}{\bar{\rho}_{T}}-\bar{\rho}_{T} \phi\left(x, y, p_{T}, t\right) \frac{\partial}{\partial p}\left(\frac{\partial \phi}{\partial t}+u \frac{\partial \phi}{\partial x}+\mathrm{v} \frac{\partial \phi}{\partial y}+\omega \frac{\partial \phi}{\partial p}-\frac{\omega}{\bar{\rho}}\right)=0
$$

$\mathrm{em} p=\mathrm{p}_{\mathrm{T}}$

É importante notar que os termos de ordem maior que a ordem quadrática $\left(\mathrm{O}\left(\phi^{2}\right)\right)$ em (2.8) foram desprezados nas equações acima ${ }^{1}$. O método aqui empregado para resolver o problema das condições de fronteira na vertical em coordenadas isobáricas é similar ao utilizado na teoria de ondas de gravidade de superfície em águas profundas (Milewski e Keller 1996), bem como no problema de ondas de gravidade-capilares (Case e Chiu 1977; McGoldrick 1965). De fato, o problema de ondas de gravidade de superfície em águas profundas é um típico exemplo de um fenômeno no qual a não linearidade surge da fronteira. Neste caso, devido à suposição de um escoamento irrotacional, o problema é descrito pela equação de Laplace no interior do domínio e as condições de fronteira na superfície livre deformada pela atividade das ondas lineares são aproximadas pelas condições em uma altura média de equilíbrio conhecida, por meio de uma expansão em série de Taylor. Entretanto, ao contrário do problema aqui analisado, como no caso das ondas de superfície em águas profundas a não linearidade na mais baixa ordem se dá por meio de quartetos de ondas, é necessário uma expansão em Taylor até a segunda ordem.

Assim, se o campo básico for conhecido, o problema formado pelas equações (2.1a), (2.1b), (2.1d) e (2.3) constitui um sistema de quatro equações a quatro incógnitas dadas por $u$, $\mathrm{v}, \omega$ e $\phi$, juntamente com as condições de fronteira na vertical dadas por (2.9). Como condições de fronteira na horizontal, é razoável supor que:

$$
\begin{aligned}
& {\left[\begin{array}{l}
u \\
\mathrm{v} \\
\phi \\
\omega
\end{array}\right]\left(x+L_{x}, y, p, t\right)=\left[\begin{array}{l}
u \\
\mathrm{v} \\
\phi \\
\omega
\end{array}\right](x, y, p, t)} \\
& \lim _{y \rightarrow \pm \infty}\left[\begin{array}{l}
u \\
\mathrm{v} \\
\phi \\
\omega
\end{array}\right](x, y, p, t)=0
\end{aligned}
$$

\footnotetext{
${ }^{1}$ Como será mostrado mais adiante, para o estudo das tríades ressonantes somente os termos retidos em (2.9) são relevantes.
} 
A periodicidade na direção zonal, dada pela condição (2.10a), está relacionada com a esfericidade da Terra, supondo que $\mathrm{L}_{\mathrm{x}}$ é o perímetro equatorial da mesma. A condição de fronteira em y (2.10b) surge da idéia de que o plano $\beta$-equatorial somente representa uma boa aproximação para os movimentos atmosféricos e oceânicos próximos ao equador. Esta condição poderia até ser enfraquecida no sentido de requerer a limitação da solução numa latitude correspondente à distância do equador aos pólos. Entretanto, exceto para o caso das perturbações associadas ao modo barotrópico, a condição (2.10b) não é restritiva, uma vez que as perturbações associadas aos modos verticais internos são bastante confinadas na região equatorial, como será mostrado mais adiante. No caso do modo externo, por outro lado, as perturbações apresentam uma considerável projeção em latitudes médias e altas, mesmo para os modos meridionais mais baixos, como também será mostrado mais adiante. Inclusive, para o modo meridional $\mathrm{n}=2$ as perturbações dos campos do vento zonal e do geopotencial correspondentes a este modo vertical apresentam uma estrutura oscilatória em y até uma latitude crítica maior que a distância do equador aos pólos, só apresentando um decaimento adiante de tal latitude crítica. De acordo com Lindzen (1967), neste caso o plano $\beta$-equatorial não representa, estritamente falando, uma boa aproximação para as autofunções das equações da água-rasa na esfera, o que poderia implicar aos resultados do presente trabalho um caráter um tanto irrealista. Entretanto, tanto nas integrações de Hoskins et al. (1977) com um modelo barotrópico, quanto nas integrações de Webster (1981; 1982), Hoskins e Karoly (1981), Kasahara e Silva Dias (1986) e vários outros, utilizando modelos atmosféricos baroclínicos, todos considerando a geometria esférica da Terra, é notável que um trem de ondas de Rossby barotrópicas gerado por uma fonte tropical de calor apresenta uma significativa propagação meridional da energia de modo a influenciar de forma bastante expressiva regiões de latitudes médias e altas. Dessa forma, embora a geometria esférica possa modificar a estrutura meridional das perturbações associadas ao modo barotrópico, não se acredita que a limitação do modelo aqui adotado em não incluir os efeitos da geometria esférica da Terra seja importante dentro dos propósitos do presente estudo, que objetiva investigar os mecanismos dinâmicos fundamentais que levam à interação entre as circulações atmosféricas tropical e extratropical, ao invés de representar de forma realista a propagação meridional da energia.

Para se estudar mais a fundo o modelo de equações primitivas dado por (2.1a), (2.1b), (2.1d) e (2.3), juntamente com as condições de fronteira (2.9) e (2.10), é conveniente analisar a magnitude dos diferentes parâmetros físicos e conseqüentemente das escalas dos diferentes termos nestas equações. Neste caso, a importância relativa dos diferentes mecanismos físicos 
é então medida pela razão entre as correspondentes escalas. Logo, é conveniente transformar as equações governantes para a escala adimensional e, a partir dessas equações na forma adimensional, é possível fazer algumas suposições com relação aos parâmetros físicos que multiplicam cada termo de tais equações com o intuito de fazer aproximações assintóticas. Isto é exatamente o que será feito na próxima seção do presente capítulo, onde será mostrado que no limite de pequenas amplitudes e/ou grandes escalas espaciais as ondas equatoriais de Rossby, Yanai, Poincaré e Kelvin constituem a solução de ordem dominante, enquanto a correção de ordem superior leva ao interessante fenômeno da ressonância não linear entre essas ondas, no qual os modos trocam energia entre si numa escala de tempo longa comparada com o período das mesmas.

\subsection{Equações Primitivas na Forma Adimensional e Aplicação do Método Assintótico de Perturbação}

Considerando U como a escala típica do campo do vento horizontal, $\Omega$ representando a escala típica de $\omega, \mathrm{L}$ como a escala característica de comprimento na horizontal, $\mathrm{p}_{0}$ como sendo a escala característica de pressão, $(\beta \mathrm{L})^{-1}$ como a escala de tempo característica e $\beta \mathrm{L}^{2} \mathrm{U}$ como a escala típica do geopotencial, é possível definir as seguintes variáveis adimensionais:

$x^{\prime}=x / L, y^{\prime}=y / L, t^{\prime}=t \beta L, u^{\prime}=u / U, v^{\prime}=v / U, \omega^{\prime}=\omega / \Omega, p^{\prime}=p / p_{0}, \phi^{\prime}=\phi /\left(\beta L^{2} U\right)$

Conseqüentemente, substituindo as relações em (2.11) nas equações (2.1a), (2.1b), (2.1d) e (2.3), obtém-se o seguinte sistema de equações primitivas na forma adimensional:

$$
\begin{aligned}
& \frac{\partial u^{\prime}}{\partial t^{\prime}}+R o\left(u^{\prime} \frac{\partial u^{\prime}}{\partial x^{\prime}}+\mathrm{v}^{\prime} \frac{\partial u^{\prime}}{\partial y^{\prime}}\right)+\Theta \omega^{\prime} \frac{\partial u^{\prime}}{\partial p^{\prime}}-y^{\prime} \mathrm{v}^{\prime}+\frac{\partial \phi^{\prime}}{\partial x^{\prime}}=0 \\
& \frac{\partial \mathrm{v}^{\prime}}{\partial t^{\prime}}+R o\left(u^{\prime} \frac{\partial \mathrm{v}^{\prime}}{\partial x^{\prime}}+\mathrm{v}^{\prime} \frac{\partial \mathrm{v}^{\prime}}{\partial y^{\prime}}\right)+\Theta \omega^{\prime} \frac{\partial \mathrm{v}^{\prime}}{\partial p^{\prime}}+y^{\prime} \mathrm{u}^{\prime}+\frac{\partial \phi^{\prime}}{\partial y^{\prime}}=0 \\
& \frac{\partial \mathrm{u}^{\prime}}{\partial x^{\prime}}+\frac{\partial \mathrm{v}^{\prime}}{\partial y^{\prime}}+\frac{\Theta}{R o} \frac{\partial \omega^{\prime}}{\partial p^{\prime}}=0
\end{aligned}
$$


$\frac{\partial}{\partial t^{\prime}} \frac{\partial \phi^{\prime}}{\partial p^{\prime}}+R o\left(u^{\prime} \frac{\partial}{\partial x^{\prime}} \frac{\partial \phi^{\prime}}{\partial p^{\prime}}+v^{\prime} \frac{\partial}{\partial y^{\prime}} \frac{\partial \phi^{\prime}}{\partial p^{\prime}}\right)+\Theta\left(\omega^{\prime} \frac{\partial^{2} \phi^{\prime}}{\partial p^{\prime 2}}+\frac{\omega^{\prime}}{p^{\prime}}(1-\kappa) \frac{\partial \phi^{\prime}}{\partial p^{\prime}}\right)+\frac{\Theta}{R o} \tilde{\widetilde{\sigma}} \omega^{\prime}=0$

sendo

$$
\widetilde{\bar{\sigma}}=\frac{\bar{\sigma} p_{0}^{2}}{\beta^{2} L^{4}}
$$

o parâmetro de estabilidade estática do estado básico na escala adimensional. Na equação (2.12) acima os parâmetros adimensionais Ro e $\Theta$ são dados por

$$
R o=\frac{U}{\beta L^{2}} \quad, \quad \Theta=\frac{\Omega}{\beta p_{0} L}
$$

Da mesma maneira, substituindo (2.11) em (2.9) obtém-se as condições de fronteira verticais na forma adimensional:

$$
\begin{aligned}
& \frac{\partial \phi^{\prime}}{\partial t^{\prime}}+R o\left(u^{\prime} \frac{\partial \phi^{\prime}}{\partial x^{\prime}}+\mathrm{v}^{\prime} \frac{\partial \phi^{\prime}}{\partial y^{\prime}}\right)+\Theta \omega^{\prime} \frac{\partial \phi^{\prime}}{\partial p^{\prime}}-\frac{\Theta}{R o} \frac{\omega^{\prime}}{\widetilde{\bar{\rho}}_{0}}+ \\
& R o \widetilde{\bar{\rho}}_{0} \phi^{\prime}\left(x^{\prime}, y^{\prime}, 1, t^{\prime}\right) \frac{\partial}{\partial p^{\prime}}\left[\frac{\partial \phi^{\prime}}{\partial t^{\prime}}+R o\left(u^{\prime} \frac{\partial \phi^{\prime}}{\partial x^{\prime}}+\mathrm{v}^{\prime} \frac{\partial \phi^{\prime}}{\partial y^{\prime}}\right)+\Theta \omega^{\prime} \frac{\partial \phi^{\prime}}{\partial p^{\prime}}-\frac{\Theta}{R o} \frac{\omega^{\prime}}{\widetilde{\widetilde{\rho}}}\right]=0
\end{aligned}
$$$$
\text { em } \mathrm{p}=1
$$

$\frac{\partial \phi^{\prime}}{\partial t^{\prime}}+R o\left(u^{\prime} \frac{\partial \phi^{\prime}}{\partial x^{\prime}}+\mathrm{v}^{\prime} \frac{\partial \phi^{\prime}}{\partial y^{\prime}}\right)+\Theta \omega^{\prime} \frac{\partial \phi^{\prime}}{\partial p^{\prime}}-\frac{\Theta}{R o} \frac{\omega^{\prime}}{\widetilde{\bar{\rho}}_{T}}-$

$R o \widetilde{\bar{\rho}}_{T} \phi^{\prime}\left(x^{\prime}, y^{\prime}, \widetilde{p}_{T}, t^{\prime}\right) \frac{\partial}{\partial p^{\prime}}\left[\frac{\partial \phi^{\prime}}{\partial t^{\prime}}+R o\left(u^{\prime} \frac{\partial \phi^{\prime}}{\partial x^{\prime}}+\mathrm{v}^{\prime} \frac{\partial \phi^{\prime}}{\partial y^{\prime}}\right)+\Theta \omega^{\prime} \frac{\partial \phi^{\prime}}{\partial p^{\prime}}-\frac{\Theta}{R o} \frac{\omega^{\prime}}{\widetilde{\rho}}\right]=0$

em $\mathrm{p}=\widetilde{p}_{T}$

onde

$$
\widetilde{\bar{\rho}}=\frac{\bar{\rho} \beta^{2} L^{4}}{P_{0}} \quad \text { e } \quad \widetilde{p}_{T}=\frac{p_{T}}{p_{0}}
$$


referem-se, respectivamente, à densidade do estado básico e à pressão "próxima" ao topo da atmosfera, ambos na forma adimensional. A partir das equações (2.12), (2.14) e (2.15) nota-se que os parâmetros adimensionais Ro e $\Theta$ representam uma medida da amplitude das perturbações e, conseqüentemente, uma medida da magnitude da não linearidade. $\mathrm{O}$ parâmetro Ro pode ser pensado como sendo o análogo na região equatorial ao número de Rossby em médias latitudes, dado que $\beta \mathrm{L}$ representa a escala típica do parâmetro de Coriolis. Este número mede a amplitude relativa das perturbações associadas ao movimento horizontal, enquanto $\Theta$ representa a medida da magnitude das perturbações associadas ao movimento vertical. A partir de (2.14) é possível verificar que, embora o escoamento atmosférico seja essencialmente não linear, não se acredita que a não linearidade seja forte para as grandes escalas. De fato, considerando as escalas típicas para os movimentos de grande-escala na atmosfera, $\mathrm{U} \approx 5 \mathrm{~m} / \mathrm{s}, \mathrm{L}=1000 \mathrm{Km}$ e $\beta=2,3 \times 10^{-11} \mathrm{~m}^{-1} \mathrm{~s}^{-1}$, tem-se que Ro $\approx \mathrm{O}\left(10^{-1}\right)$. Logo, soluções de ondas fracamente não lineares parecem razoáveis na atmosfera. Inclusive, existem evidências observacionais que reforçam a idéia de que movimentos ondulatórios de grandeescala são, de fato, existentes na atmosfera (Yanai e Maruyama 1966; Maruyama 1967; Wallace e Kousky 1968; Pires et al. 1987; Wheeler e Kiladis 1999; e vários outros). Conseqüentemente, torna-se conveniente e bastante oportuna a aplicação da teoria clássica de perturbação assintótica com o intuito de analisar a natureza desses movimentos atmosféricos neste limite de pequenas amplitudes. Assim, para a aplicação da teoria clássica de perturbação assintótica são necessárias primeiramente as seguintes suposições:

(i) Supõe-se que o número de Rossby é um parâmetro pequeno, tal que

$$
\text { Ro }=\varepsilon, \quad \text { onde } \quad 0<\varepsilon<<1
$$

(ii) Supõe-se que, a priori, não há uma distinção significativa entre as ordens de grandeza das amplitudes das perturbações associadas aos movimentos verticais e horizontais. Neste caso, tem-se que

$$
\Theta / \text { Ro }=F \quad \text {, onde } \quad F=O(1)
$$

Na realidade, a suposição (ii) não é exatamente necessária para a aplicação de uma teoria de perturbação assintótica, e sim conveniente para os propósitos deste estudo, que tem 
por objetivo analisar as interações não lineares fracas entre os diferentes tipos de ondas existentes na atmosfera, sem discriminar, a priori, nenhum tipo de onda em particular como sendo de ordem dominante. Se $\Theta /$ Ro fosse também pequeno, a teoria seria desenvolvida em termos de dois parâmetros adimensionais, ao invés de em termos de apenas um único parâmetro. Conseqüentemente, é possível observar a partir das equações (2.12) que a solução de ordem dominante neste caso (ou de mais baixa ordem), além de linear, seria caracterizada por um escoamento horizontal não divergente. Logo, somente ondas de Rossby barotrópicas não-divergentes constituiriam a solução de ordem dominante, ao invés de todos os tipos de ondas como é o caso do presente trabalho, como mostrado mais adiante, e a solução de ordem superior daria as correções devidas tanto à divergência finita quanto à amplitude finita. Uma análise assintótica para o caso Ro $<<1$ e $\Theta /$ Ro $<<1$ seria similar à realizada por Milewski e Tabak (1999). Milewski e Tabak, adotando um modelo representativo de uma camada de fluido homogêneo, com uma superfície livre, e sobre a Terra em rotação, desenvolveram um modelo reduzido que descreve a evolução fracamente não linear de ondas longas a partir de uma análise de perturbação assintótica baseada numa expansão em série de potências de dois parâmetros adimensionais pequenos: um que multiplica os termos advectivos na equação do momento horizontal e o outro que multiplica os termos da aceleração na equação do momento vertical. Neste caso, Milewski e Tabak obtiveram tanto as correções de amplitude finita quanto as correções de profundidade finita à solução de ordem dominante, dada pela solução das equações da água rasa linearizadas. Eles mostram que tais correções implicam numa pequena dispersão das ondas de Kelvin, levando a uma equação do tipo K-dV com soluções em forma de ondas solitárias. Com isso, uma análise similar para o sistema de equações primitivas dado por (2.12) seria uma interessante sugestão para pesquisa futura.

Feitas as suposições (i) e (ii), o sistema de equações governantes dado por (2.12) pode ser escrito da seguinte forma:

$$
\begin{aligned}
& \frac{\partial u}{\partial t}+\varepsilon\left(u \frac{\partial u}{\partial x}+\mathrm{v} \frac{\partial u}{\partial y}+F \omega \frac{\partial u}{\partial p}\right)-y \mathrm{v}+\frac{\partial \phi}{\partial x}=0 \\
& \frac{\partial \mathrm{v}}{\partial t}+\varepsilon\left(u \frac{\partial \mathrm{v}}{\partial x}+\mathrm{v} \frac{\partial \mathrm{v}}{\partial y}+F \omega \frac{\partial \mathrm{v}}{\partial p}\right)+y \mathrm{u}+\frac{\partial \phi}{\partial y}=0 \\
& \frac{\partial \mathrm{u}}{\partial x}+\frac{\partial \mathrm{v}}{\partial y}+F \frac{\partial \omega}{\partial p}=0
\end{aligned}
$$


$\frac{\partial}{\partial t} \frac{\partial \phi}{\partial p}+\varepsilon\left[u \frac{\partial}{\partial x} \frac{\partial \phi}{\partial p}+\mathrm{v} \frac{\partial}{\partial y} \frac{\partial \phi}{\partial p}+F \omega \frac{\partial^{2} \phi}{\partial p^{2}}+\frac{F \omega}{p}(1-\kappa) \frac{\partial \phi}{\partial p}\right]+F \widetilde{\widetilde{\sigma} \omega}=0$

onde o sobre-índice ", " referente às grandezas adimensionais foi ocultado por simplicidade. Da mesma maneira, as equações referentes às condições de fronteira na vertical tornam-se:

$$
\begin{aligned}
& \frac{\partial \phi}{\partial t}+\varepsilon\left(u \frac{\partial \phi}{\partial x}+\mathrm{v} \frac{\partial \phi}{\partial y}+F \omega \frac{\partial \phi}{\partial p}\right)-\frac{F \omega}{\widetilde{\bar{\rho}}_{0}}+\varepsilon \widetilde{\bar{\rho}_{0}} \phi(x, y, 1, t) \frac{\partial}{\partial p}\left[\frac{\partial \phi}{\partial t} \varepsilon\left(u \frac{\partial \phi}{\partial x}+\mathrm{v} \frac{\partial \phi}{\partial y}+F \omega \frac{\partial \phi}{\partial p}\right)-\frac{F \omega}{\widetilde{\bar{\rho}}}\right]=0 \\
& \mathrm{em} \mathrm{p}=1
\end{aligned}
$$

$$
\begin{aligned}
& \frac{\partial \phi}{\partial t}+\varepsilon\left(u \frac{\partial \phi}{\partial x}+\mathrm{v} \frac{\partial \phi}{\partial y}+F \omega \frac{\partial \phi}{\partial p}\right)-\frac{F \omega}{\widetilde{\widetilde{\rho}}_{T}}-\varepsilon \widetilde{\bar{\rho}}_{T} \phi\left(x, y, \widetilde{p}_{T}, t\right) \frac{\partial}{\partial p}\left[\frac{\partial \phi}{\partial t} \varepsilon\left(u \frac{\partial \phi}{\partial x}+\mathrm{v} \frac{\partial \phi}{\partial y}+F \omega \frac{\partial \phi}{\partial p}\right)-\frac{F \omega}{\widetilde{\bar{\rho}}}\right]=0 \\
& \operatorname{em~} \mathrm{p}=\tilde{p}_{T}
\end{aligned}
$$

onde nas equações acima o sobre-índice “, ” também foi ocultado por simplicidade. Dividindo a equação da termodinâmica (2.18d) por $\widetilde{\bar{\sigma}}$, derivando a equação resultante com relação a p e usando a equação da continuidade (2.18c), obtém-se:

$$
\frac{\partial}{\partial t} \frac{\partial}{\partial p}\left(\frac{1}{\widetilde{\widetilde{\sigma}}} \frac{\partial \phi}{\partial p}\right)+\varepsilon \frac{\partial}{\partial p}\left[\frac{u}{\widetilde{\widetilde{\sigma}}} \frac{\partial}{\partial x} \frac{\partial \phi}{\partial p}+\frac{\mathrm{v}}{\widetilde{\widetilde{\sigma}}} \frac{\partial}{\partial y} \frac{\partial \phi}{\partial p}+\frac{F \omega}{\widetilde{\widetilde{\sigma}}} \frac{\partial^{2} \phi}{\partial p^{2}}+\frac{F \omega}{\widetilde{\sigma} p}(1-\kappa) \frac{\partial \phi}{\partial p}\right]-\left(\frac{\partial u}{\partial x}+\frac{\partial \mathrm{v}}{\partial y}\right)=0
$$

Assim, a análise do sistema formado pelas equações (2.18a-b) e a equação (2.20), com as condições de fronteira dadas por (2.10) e (2.19), é feita usando-se a teoria de perturbação assintótica baseada no método de múltiplas escalas temporais. Tal método supõe uma separação entre duas escalas temporais distintas: uma escala rápida de tempo, representada por $\mathrm{t}$, e outra escala longa de tempo, representada por $\tau=\varepsilon t$. Formalmente, permite-se que as variáveis de estado do modelo sejam funções de ambas escalas temporais, de tal forma que as derivadas temporais sejam substituídas por

$$
\frac{\partial}{\partial t} \rightarrow \frac{\partial}{\partial t}+\varepsilon \frac{\partial}{\partial \tau}+O\left(\varepsilon^{2}\right)
$$


Dessa forma, sendo as variáveis de estado do modelo dependentes de $\varepsilon$ e sendo este um parâmetro pequeno, supondo que as variáveis de estado sejam funções analíticas de $\varepsilon$, a hipótese do presente trabalho que será justificada à posteriori é dada pela seguinte expansão assintótica em série de potências de $\varepsilon$ :

$$
\begin{aligned}
& u=u^{(0)}(x, y, p, t, \varepsilon t)+\varepsilon u^{(1)}(x, y, p, t, \varepsilon t)+O\left(\varepsilon^{2}\right) \\
& v=v^{(0)}(x, y, p, t, \varepsilon t)+\varepsilon v^{(1)}(x, y, p, t, \varepsilon t)+O\left(\varepsilon^{2}\right) \\
& \phi=\phi^{(0)}(x, y, p, t, \varepsilon t)+\varepsilon \phi^{(1)}(x, y, p, t, \varepsilon t)+O\left(\varepsilon^{2}\right) \\
& \omega=\omega^{(0)}(x, y, p, t, \varepsilon t)+\varepsilon \omega^{(1)}(x, y, p, t, \varepsilon t)+O\left(\varepsilon^{2}\right)
\end{aligned}
$$

Como será destacado mais adiante, o método de múltiplas escalas temporais representado por (2.21) é necessário para eliminar as chamadas soluções seculares que surgem no problema de $\mathrm{O}(\varepsilon)$ quando os termos não lineares aplicados nas soluções de mais baixa ordem excitam ressonantemente algum termo que constitui uma solução do problema linear. Dessa forma, o método de múltiplas escalas temporais representado por (2.21) é necessário para garantir a validade da expansão assintótica dada por (2.22).

Assim, substituindo as variáveis $\mathrm{u}, \mathrm{v}, \phi$ e $\omega$ e as derivadas temporais nas equações (2.18a-b) e (2.20) por suas respectivas expansões assintóticas dadas por (2.22) e (2.21), obtém-se:

$$
\begin{aligned}
& \frac{\partial u^{(0)}}{\partial t}+\varepsilon \frac{\partial u^{(1)}}{\partial t}+\varepsilon \frac{\partial u^{(0)}}{\partial \tau}+\varepsilon\left(u^{(0)} \frac{\partial u^{(0)}}{\partial x}+\mathrm{v}^{(0)} \frac{\partial u^{(0)}}{\partial y}+F \omega^{(0)} \frac{\partial u^{(0)}}{\partial p}\right)-y \mathrm{v}^{(0)}-\varepsilon y \mathrm{v}^{(1)}+\frac{\partial \phi^{(0)}}{\partial x}+ \\
& \varepsilon \frac{\partial \phi^{(1)}}{\partial x}+O\left(\varepsilon^{2}\right)=0
\end{aligned}
$$

$$
\begin{aligned}
& \frac{\partial \mathrm{v}^{(0)}}{\partial t}+\varepsilon \frac{\partial \mathrm{v}^{(1)}}{\partial t}+\varepsilon \frac{\partial \mathrm{v}^{(0)}}{\partial \tau}+\varepsilon\left(u^{(0)} \frac{\partial \mathrm{v}^{(0)}}{\partial x}+\mathrm{v}^{(0)} \frac{\partial \mathrm{v}^{(0)}}{\partial y}+F \omega^{(0)} \frac{\partial \mathrm{v}^{(0)}}{\partial p}\right)+y \mathrm{u}^{(0)}+\varepsilon y \mathrm{u}^{(1)}+\frac{\partial \phi^{(0)}}{\partial y}+ \\
& \varepsilon \frac{\partial \phi^{(1)}}{\partial y}+O\left(\varepsilon^{2}\right)=0
\end{aligned}
$$




$$
\begin{aligned}
& \frac{\partial}{\partial t} \frac{\partial}{\partial p}\left(\frac{1}{\widetilde{\widetilde{\sigma}}} \frac{\partial \phi^{(0)}}{\partial p}\right)+\varepsilon \frac{\partial}{\partial t} \frac{\partial}{\partial p}\left(\frac{1}{\widetilde{\widetilde{\sigma}}} \frac{\partial \phi^{(1)}}{\partial p}\right)+\varepsilon \frac{\partial}{\partial \tau} \frac{\partial}{\partial p}\left(\frac{1}{\widetilde{\widetilde{\sigma}}} \frac{\partial \phi^{(0)}}{\partial p}\right)+ \\
& \varepsilon \frac{\partial}{\partial p}\left[\frac{u^{(0)}}{\widetilde{\widetilde{\sigma}}} \frac{\partial}{\partial x} \frac{\partial \phi^{(0)}}{\partial p}+\frac{\mathrm{v}^{(0)}}{\widetilde{\widetilde{\sigma}}} \frac{\partial}{\partial y} \frac{\partial \phi^{(0)}}{\partial p}+\frac{F \omega^{(0)}}{\widetilde{\widetilde{\sigma}}} \frac{\partial^{2} \phi^{(0)}}{\partial p^{2}}+\frac{F \omega^{(0)}}{\widetilde{\widetilde{\sigma}} p}(1-\kappa) \frac{\partial \phi^{(0)}}{\partial p}\right]- \\
& \left(\frac{\partial u^{(0)}}{\partial x}+\frac{\partial \mathrm{v}^{(0)}}{\partial y}\right)-\varepsilon\left(\frac{\partial u^{(1)}}{\partial x}+\frac{\partial \mathrm{v}^{(1)}}{\partial y}\right)+O\left(\varepsilon^{2}\right)=0
\end{aligned}
$$

Da mesma maneira, inserindo as expansões assintóticas dadas por (2.22) e (2.21) em (2.19), pode-se reescrever as condições de fronteira na vertical da seguinte forma:

$$
\begin{aligned}
& \frac{\partial \phi^{(0)}}{\partial t}+\varepsilon \frac{\partial \phi^{(1)}}{\partial t}+\varepsilon \frac{\partial \phi^{(0)}}{\partial \tau}+\varepsilon\left(u^{(0)} \frac{\partial \phi^{(0)}}{\partial x}+\mathrm{v}^{(0)} \frac{\partial \phi^{(0)}}{\partial y}+F \omega^{(0)} \frac{\partial \phi^{(0)}}{\partial p}\right)-\frac{F \omega^{(0)}}{\widetilde{\bar{\rho}}_{0}}-\varepsilon \frac{F \omega^{(1)}}{\widetilde{\bar{\rho}}_{0}}+ \\
& \varepsilon \widetilde{\bar{\rho}}_{0} \phi^{(0)}(x, y, 1, t) \frac{\partial}{\partial p}\left[\frac{\partial \phi^{(0)}}{\partial t}-\frac{F \omega^{(0)}}{\widetilde{\bar{\rho}}}\right]+O\left(\varepsilon^{2}\right)=0 \\
& \mathrm{em} \mathrm{p}=1
\end{aligned}
$$

$$
\begin{aligned}
& \frac{\partial \phi^{(0)}}{\partial t}+\varepsilon \frac{\partial \phi^{(1)}}{\partial t}+\varepsilon \frac{\partial \phi^{(0)}}{\partial \tau}+\varepsilon\left(u^{(0)} \frac{\partial \phi^{(0)}}{\partial x}+\mathrm{v}^{(0)} \frac{\partial \phi^{(0)}}{\partial y}+F \omega^{(0)} \frac{\partial \phi^{(0)}}{\partial p}\right)-\frac{F \omega^{(0)}}{\widetilde{\bar{\rho}}_{T}}-\varepsilon \frac{F \omega^{(1)}}{\widetilde{\bar{\rho}}_{T}}- \\
& \varepsilon \widetilde{\bar{\rho}}_{T} \phi^{(0)}\left(x, y, \widetilde{p}_{T}, t\right) \frac{\partial}{\partial p}\left[\frac{\partial \phi^{(0)}}{\partial t}-\frac{F \omega^{(0)}}{\widetilde{\bar{\rho}}}\right]+O\left(\varepsilon^{2}\right)=0 \\
& \mathrm{em} \mathrm{p}=\tilde{p}_{T}
\end{aligned}
$$

Assim, como pode ser observado a partir das equações (2.23) e (2.24), para cada potência de $\varepsilon$ tem-se um problema a ser resolvido para os coeficientes das expansões em série em (2.22).

\subsubsection{O Problema de $\mathrm{O}(1)$}

A partir das equações (2.23) e (2.24), observa-se facilmente que o problema de $\mathrm{O}(1)$, que engloba todos os termos com $\varepsilon^{0}$, é dado pelo seguinte sistema de equações diferenciais parciais linear e homogêneo: 


$$
\begin{array}{r}
\frac{\partial u^{(0)}}{\partial t}-y \mathrm{v}^{(0)}+\frac{\partial \phi^{(0)}}{\partial x}=0 \\
\frac{\partial \mathrm{v}^{(0)}}{\partial t}+y \mathrm{u}^{(0)}+\frac{\partial \phi^{(0)}}{\partial y}=0 \\
\frac{\partial}{\partial t} \frac{\partial}{\partial p}\left(\frac{1}{\widetilde{\sigma}} \frac{\partial \phi^{(0)}}{\partial p}\right)-\left(\frac{\partial u^{(0)}}{\partial x}+\frac{\partial \mathrm{v}^{(0)}}{\partial y}\right)=0
\end{array}
$$

com as condições de fronteira na vertical dadas por

$$
\begin{array}{ll}
\frac{\partial \phi^{(0)}}{\partial t}-\frac{F \omega^{(0)}}{{\widetilde{\rho_{0}}}_{0}}=0 & \text { em } \mathrm{p}=1 \\
\frac{\partial \phi^{(0)}}{\partial t}-\frac{F \omega^{(0)}}{\widetilde{\bar{\rho}}_{T}}=0 & \text { em } \mathrm{p}=\widetilde{p}_{T}
\end{array}
$$

Dado que o problema acima é linear, é possível resolvê-lo através do método de separação de variáveis. Logo, usando a seguinte separação de variáveis:

$$
\left[\begin{array}{l}
u^{(0)}(x, y, p, t, \tau) \\
\mathrm{v}^{(0)}(x, y, p, t, \tau) \\
\phi^{(0)}(x, y, p, t, \tau)
\end{array}\right]=\left[\begin{array}{l}
\hat{u}(x, y, t, \tau) \\
\hat{\mathrm{v}}(x, y, t, \tau) \\
\hat{\phi}(x, y, t, \tau)
\end{array}\right] G(p)
$$

e substituindo esta expressão em (2.25), obtém-se

$$
\begin{aligned}
& \left(\frac{\partial \hat{u}}{\partial t}-y \hat{\mathrm{v}}+\frac{\partial \hat{\phi}}{\partial x}\right) G=0 \\
& \left(\frac{\partial \hat{\mathrm{v}}}{\partial t}+y \hat{\mathrm{u}}+\frac{\partial \hat{\phi}}{\partial y}\right) G=0 \\
& \frac{\partial \hat{\phi}}{\partial t} \frac{d}{d p}\left(\frac{1}{\widetilde{\widetilde{\sigma}}} \frac{d G}{d p}\right)-\left(\frac{\partial \hat{u}}{\partial x}+\frac{\partial \hat{\mathrm{v}}}{\partial y}\right) G=0
\end{aligned}
$$

De (2.28c), segue que 


$$
\frac{\frac{\partial \hat{\phi}}{\partial t}}{\left(\frac{\partial \hat{u}}{\partial x}+\frac{\partial \hat{\mathrm{v}}}{\partial y}\right)}=\frac{G}{\frac{d}{d p}\left(\frac{1}{\widetilde{\widetilde{\sigma}}} \frac{d G}{d p}\right)}=-c^{2}
$$

onde c é a constante de separação. O sinal negativo no lado direito de (2.29) é necessário para que a solução satisfaça às condições de fronteira na vertical, como será mostrado em seguida. De (2.29) segue que a estrutura horizontal é governada por um sistema de equações similar ao das equações da água-rasa com a altura equivalente dada por $\mathrm{c}^{2} / \mathrm{g}$, ou seja,

$$
\begin{aligned}
& \frac{\partial \hat{u}}{\partial t}-y \hat{\mathrm{v}}+\frac{\partial \hat{\phi}}{\partial x}=0 \\
& \frac{\partial \hat{\mathrm{v}}}{\partial t}+y \hat{\mathrm{u}}+\frac{\partial \hat{\phi}}{\partial y}=0 \\
& \frac{\partial \hat{\phi}}{\partial t}+c^{2}\left(\frac{\partial \hat{u}}{\partial x}+\frac{\partial \hat{\mathrm{v}}}{\partial y}\right)=0
\end{aligned}
$$

enquanto a equação que governa a estrutura vertical é dada por:

$$
\frac{d}{d p}\left(\frac{1}{\widetilde{\sigma}} \frac{d G}{d p}\right)+\frac{1}{c^{2}} G=0
$$

Da mesma maneira, a equação da termodinâmica (2.18d) para a $O(1)$ é dada por

$$
\frac{\partial}{\partial t} \frac{\partial \phi^{(0)}}{\partial p}+F \widetilde{\bar{\sigma}} \omega^{(0)}=0
$$

Assim, aplicando a equação (2.32) acima em $p=1$ e em $p=p_{\mathrm{T}}$, usando as condições de fronteira (2.26) e assumindo a separação de variáveis (2.27), segue que as condições de fronteira na vertical podem ser expressas da seguinte forma: 


$$
\frac{d G}{d p}+\widetilde{\widetilde{\sigma}} \widetilde{\rho} G=0 \quad \text { em } \mathrm{p}=1 \text { e } \quad \text { em } \mathrm{p}=\tilde{p}_{T}
$$

A equação da estrutura vertical (2.31), juntamente com as condições de fronteira dadas por (2.33), constitui um problema de Sturm-Liouville. Supondo ainda que o parâmetro de estabilidade estática $\widetilde{\widetilde{\sigma}}$ seja constante com a pressão, a solução geral de (2.31) pode ser escrita da seguinte forma:

$$
G(p)=A \cos (\lambda p)+B \operatorname{sen}(\lambda p)
$$

sendo $\lambda=\frac{\sqrt{\widetilde{\sigma}}}{c}$. Substituindo (2.34) nas condições de fronteira dadas por (2.33), obtém-se:

$$
\left[\begin{array}{cc}
\widetilde{\sigma} \widetilde{\rho}_{0} \cos \lambda-\lambda \operatorname{sen} \lambda & \widetilde{\sigma} \bar{\rho}_{0} \operatorname{sen} \lambda+\lambda \cos \lambda \\
\widetilde{\sigma} \widetilde{\rho}_{T} \cos \left(\lambda \widetilde{p}_{T}\right)-\lambda \operatorname{sen}\left(\lambda \widetilde{p}_{T}\right) & \widetilde{\sigma} \widetilde{\rho}_{T} \operatorname{sen}\left(\lambda \widetilde{p}_{T}\right)+\lambda \cos \left(\lambda \widetilde{p}_{T}\right)
\end{array}\right]\left[\begin{array}{l}
A \\
B
\end{array}\right]=\left[\begin{array}{l}
0 \\
0
\end{array}\right]
$$

Assim, a partir de (2.35) segue que os autovalores $\lambda$ são determinados a partir da seguinte equação característica:

$$
\lambda^{2} \operatorname{sen}\left[\left(1-\widetilde{p}_{T}\right) \lambda\right]-\lambda \widetilde{\widetilde{\sigma}}\left(\widetilde{\bar{\rho}}_{0}-\widetilde{\bar{\rho}}_{T}\right) \cos \left[\left(1-\widetilde{p}_{T}\right) \lambda\right]+\widetilde{\bar{\sigma}}^{2} \widetilde{\bar{\rho}}_{T} \widetilde{\bar{\rho}}_{0} \operatorname{sen}\left[\left(1-\widetilde{p}_{T}\right) \lambda\right]=0
$$

A partir de (2.35) também é possível escrever a constante B em função de A. Logo, as autofunções são dadas por

$$
G_{m}(p)=A\left[\cos \left(\lambda_{m} p\right)+\gamma_{m} \operatorname{sen}\left(\lambda_{m} p\right)\right]
$$

onde A é uma constante arbitrária e

$$
\gamma_{m}=\frac{\lambda_{m} \operatorname{sen} \lambda_{m}-\widetilde{\widetilde{\sigma} \tilde{\rho}_{0}} \cos \lambda_{m}}{\widetilde{\sigma}_{0} \operatorname{sen} \lambda_{m}+\lambda_{m} \cos \lambda_{m}}
$$


Uma vez que a equação da estrutura vertical (2.31) juntamente com as condições de contorno dadas por (2.33) constituem um problema de Sturm-Liouville, os autovalores $\lambda_{\mathrm{m}}, \mathrm{m}$ $=0,1,2,3, \ldots$, formam uma seqüência infinita enumerável com $\lambda_{0}<\lambda_{1}<\lambda_{2}<\lambda_{3}<\ldots<\lambda_{\mathrm{m}-1}<\lambda_{\mathrm{m}}$ $<\ldots$. e as autofunções $G_{m}(p)$ dadas por (2.37) formam um conjunto ortogonal e completo no espaço das funções de quadrado integrável no domínio $\left[\widetilde{p}_{T}, 1\right]$. Logo, tem-se que

$$
\int_{\widetilde{p}_{T}}^{1} G_{m}(p) G_{n}(p) d p=\left\{\begin{array}{ccc}
0 & \text { se } & m \neq n \\
\left\|G_{m}(p)\right\|^{2} & \text { se } & m=n
\end{array}\right\}
$$

onde $\left\|G_{m}(p)\right\|^{2}$ refere-se à norma das autofunções, dada por

$$
\begin{aligned}
& \left\|G_{m}\right\|^{2}=\int_{\widetilde{p}_{T}}^{1} G_{m}(p)^{2} d p=\int_{\widetilde{p}_{T}}^{1}\left[\cos \left(\lambda_{m} p\right)+\gamma_{m} \operatorname{sen}\left(\lambda_{m} p\right)\right]^{2} d p=\left(\frac{1+\gamma_{m}{ }^{2}}{2}\right)\left(1-\widetilde{p}_{T}\right)+ \\
& \left(\frac{1-\gamma_{m}{ }^{2}}{4 \lambda_{m}}\right)\left[\operatorname{sen}\left(2 \lambda_{m}\right)-\operatorname{sen}\left(2 \lambda_{m} \widetilde{p}_{T}\right)\right]+\frac{\gamma_{m}}{2 \lambda_{m}}\left[\cos \left(2 \lambda_{m} \widetilde{p}_{T}\right)-\cos \left(2 \lambda_{m}\right)\right]
\end{aligned}
$$

Com isso, dado um estado básico caracterizado pelos perfis $\bar{\rho}(\mathrm{p})$ e $\overline{\mathrm{T}}(\mathrm{p})$, os valores de $\tilde{\bar{\sigma}}, \widetilde{\bar{\rho}}_{0}$ e $\widetilde{\bar{\rho}}_{T}$ obtidos por meio das expressões (2.13) e (2.16) são utilizados para a determinação dos autovalores $\lambda_{\mathrm{m}}$ a partir da equação transcendental (2.36) e, conseqüentemente, as autofunções $\mathrm{G}_{\mathrm{m}}(\mathrm{p})$ são calculadas por meio de (2.37) e (2.38). Para a determinação dos autovalores $\lambda_{\mathrm{m}}$ da equação da estrutura vertical foi utilizado o método iterativo da Bissecção ou da Dicotomia no cálculo das raízes de (2.36). O autovalor $\lambda=0$, que trivialmente satisfaz à equação (2.36), não tem significado físico (ver Apêndice B). Logo, este autovalor nulo é desprezado na presente análise e, conseqüentemente, o autovalor que daqui a diante será associado à $\mathrm{m}=0$ é dado pela menor raiz $\lambda \neq 0$ da equação transcendental (2.36).

Para a determinação dos perfis de densidade e temperatura referentes ao estado básico, inicialmente escolhe-se um valor de $\bar{\sigma}$ típico para a troposfera e, utilizando a equação (2.2) e a equação $\bar{\sigma}=\mathrm{S}_{\mathrm{p}} \mathrm{R} / \mathrm{p}$ para $\bar{\sigma}$ constante, determina-se o perfil $\overline{\mathrm{T}}(\mathrm{p})$ e, finalmente, obtém-se o perfil $\bar{\rho}(\mathrm{p})$ utilizando a equação do estado para um gás ideal, ou seja, $\bar{\rho}=\mathrm{p} /(\mathrm{R} \overline{\mathrm{T}})$. É importante observar que a determinação de $\overline{\mathrm{T}}(\mathrm{p})$ a partir do valor de $\bar{\sigma}$ requer a resolução de uma equação diferencial ordinária de primeira ordem com coeficientes variáveis. Os detalhes desta metodologia são apresentados no Apêndice A. Os perfis de $\bar{T}(p)$ e $\bar{\rho}(p)$ 
obtidos e utilizados neste trabalho são mostrados na Fig. 2.1 para $\bar{\sigma}=2 \times 10^{-6} \mathrm{~m}^{4} \mathrm{~s}^{2} \mathrm{Kg}^{-2}$. Já os valores de $\lambda_{\mathrm{m}}$ e $\mathrm{c}_{\mathrm{m}}$ calculados a partir desses parâmetros são mostrados na Tabela 2.1. Os valores de $c_{m} \beta \mathrm{L}^{2}$, que apresentam dimensão de velocidade, também são dispostos na Tabela 2.1 .

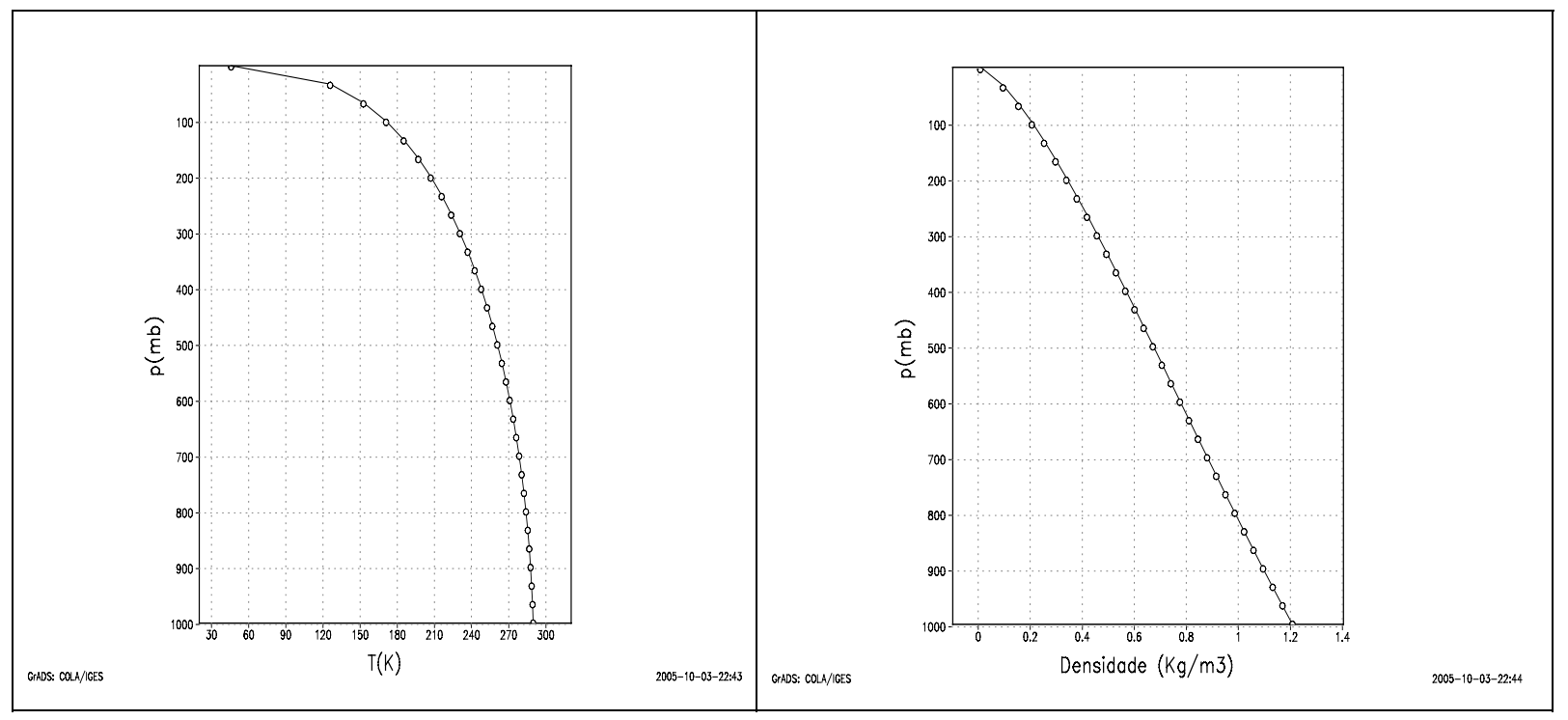

(a)

(b)

Figura 2.1: Perfis de $\overline{\mathrm{T}}(\mathrm{p})(\mathrm{a})$ e $\bar{\rho}(\mathrm{p})(\mathrm{b})$ utilizados no presente trabalho para $\bar{\sigma}=2 \times 10^{-6}$ $\mathrm{m}^{4} \mathrm{~s}^{2} \mathrm{Kg}^{-2}$.

Tabela 2.1: Valores de $\lambda_{\mathrm{m}}, \mathrm{c}_{\mathrm{m}}$ e $\mathrm{c}_{\mathrm{m}} \beta \mathrm{L}^{2}\left(\operatorname{com} \beta=2,3 \times 10^{-11} \mathrm{~m}^{-1} \mathrm{~s}^{-1}\right.$ e $\left.\mathrm{L}=1000 \mathrm{Km}\right)$ para os primeiros 5 modos verticais, $\mathrm{m}=0,1,2,3 \mathrm{e} 4$, para $\mathrm{p}_{0}=1000 \mathrm{hPa}, \mathrm{p}_{\mathrm{T}}=100 \mathrm{hPa}, \bar{\sigma}=2 \times 10^{-6}$ $\mathrm{m}^{4} \mathrm{~s}^{2} \mathrm{Kg}^{-2}$ e os perfis de $\overline{\mathrm{T}}(\mathrm{p})$ e $\bar{\rho}(\mathrm{p})$ mostrados na Fig. 2.1 .

\begin{tabular}{|c|c|c|c|}
\hline $\mathrm{m}$ & $\lambda_{\mathrm{m}}$ (adimensional) & $\mathrm{c}_{\mathrm{m}}$ (adimensional) & $\mathrm{c}_{\mathrm{m}} \beta \mathrm{L}^{2}(\mathrm{~m} / \mathrm{s})$ \\
\hline 0 & 0,44742657 & 13,843201 & 316,0772 \\
\hline 1 & 3,553075 & 1,7432269 & 39,802524 \\
\hline 2 & 7,0129829 & 0,8831928 & 20,165649 \\
\hline 3 & 10,4931446 & 0,59027263 & 13,4775 \\
\hline 4 & 13,9785263 & 0,443095 & 10,117043 \\
\hline
\end{tabular}


As estruturas das autofunções associadas aos autovalores mostrados na Tabela 2.1 são ilustradas na Figura 2.2. O modo $\mathrm{m}=0$ (comumente referido como modo externo) apresenta uma estrutura praticamente constante ao longo de toda a troposfera. Logo, as perturbações dos campos do vento e do geopotencial associadas a este modo vertical preservam a mesma fase com a altura ao longo de toda a troposfera e, por esta razão, este modo também é usualmente chamado de modo barotrópico.

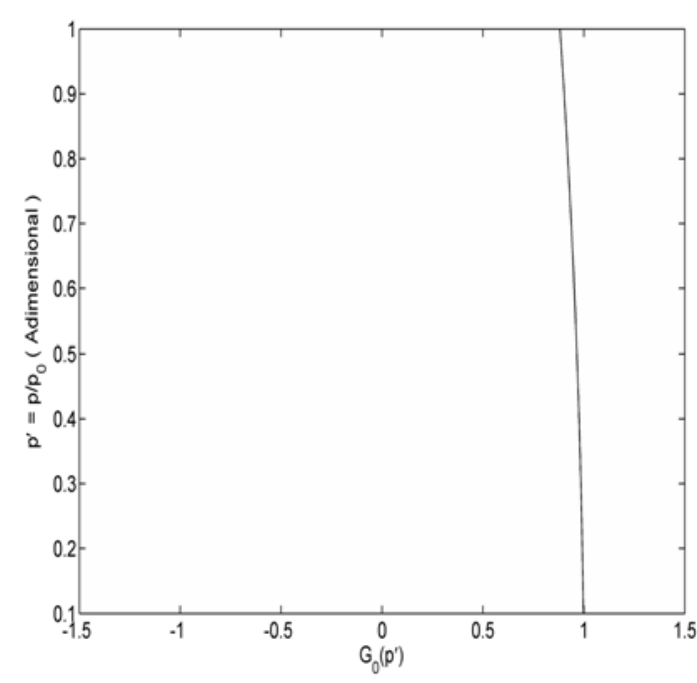

(a)

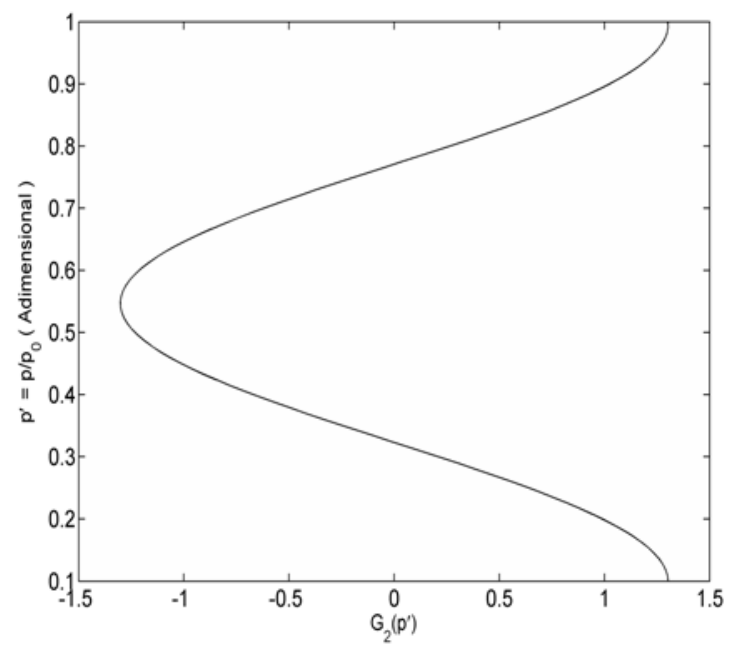

(c)

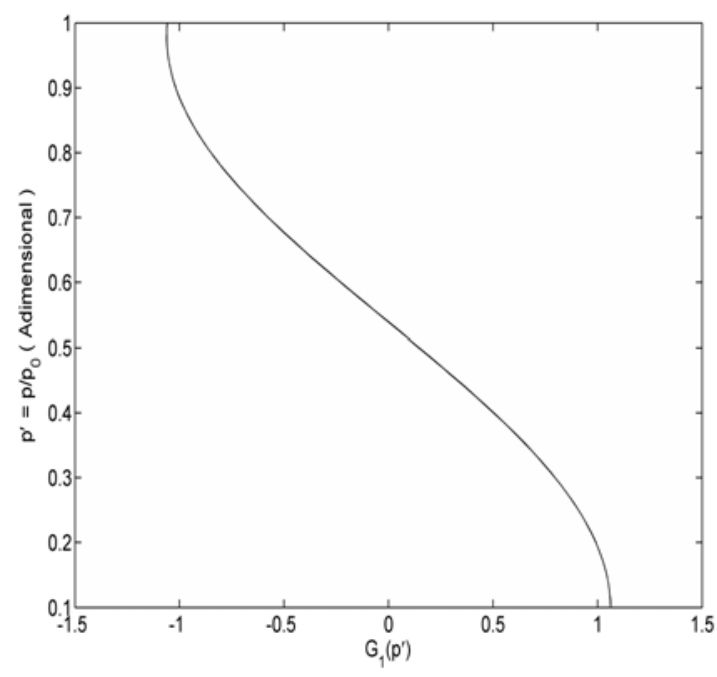

(b)

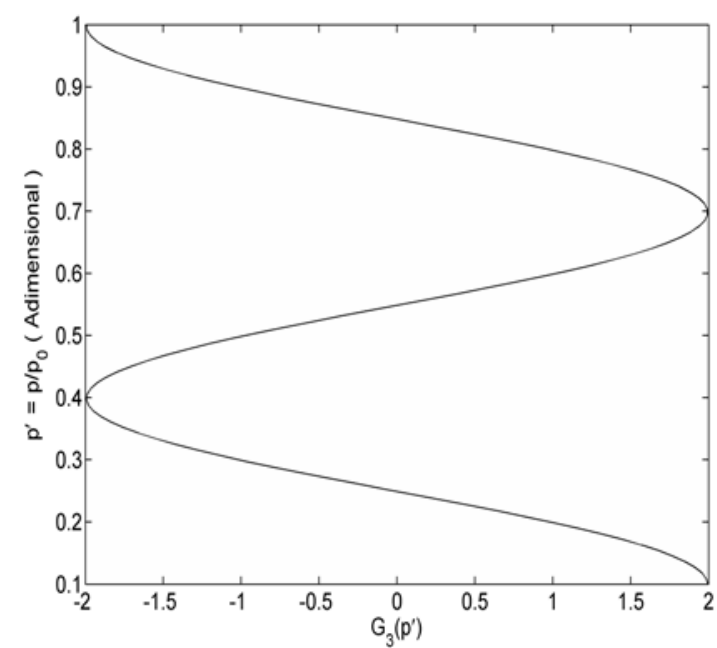

(d) 


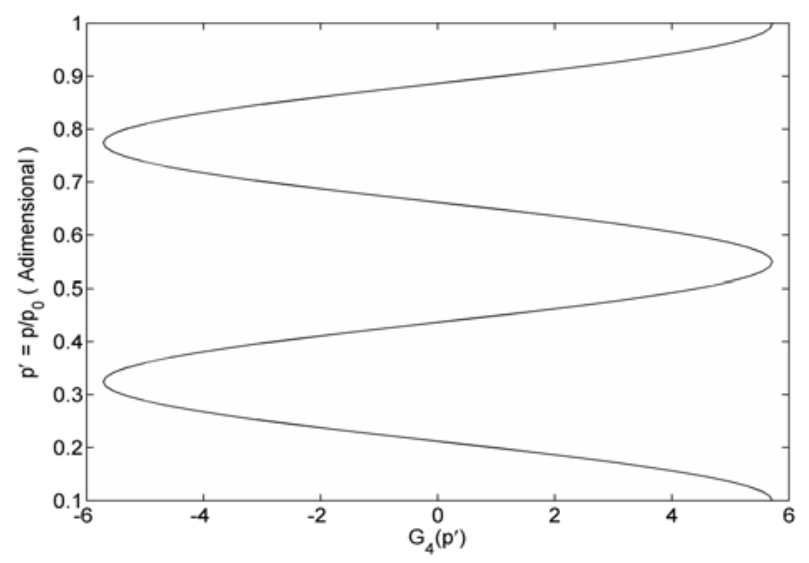

(e)

Figura 2.2: Estrutura das autofunções da equação da estrutura vertical (2.31) sujeita às condições de fronteira (2.33) para: (a) $\mathrm{m}=0$, (b) $\mathrm{m}=1$, (c) $\mathrm{m}=2$, (d) $\mathrm{m}=3$ e (e) $\mathrm{m}=4$.

A partir da Figura 2.2 também é possível observar que a autofunção $G_{1}(p)$ apresenta uma única inversão de fase com a altura ao longo da troposfera, ocorrendo em torno de $500 \mathrm{hPa}$. Conseqüentemente, a divergência horizontal associada a este modo se anula na média troposfera, apresentando uma inversão de fase entre a alta e a baixa troposfera. Este modo vertical, referido como primeiro modo baroclínico, corresponde ao modo dominante na região tropical. Esta dominância se deve ao fato de que o aquecimento diabático resultante da liberação de calor latente associada à convecção úmida organizada nos trópicos, que corresponde à principal fonte de energia para o escoamento atmosférico na região tropical, é tipicamente intenso na média troposfera e apresenta valores pequenos na superfície e na tropopausa (Yanai et al. 1973). Logo, este aquecimento convectivo apresenta uma estrutura vertical que é basicamente projetada no modo vertical $m=1$. Esta dominância do modo $m=1$ na representação da estrutura vertical do escoamento atmosférico na região tropical é verificada observacionalmente por Silva Dias e Bonatti (1995). Conforme m aumenta, os modos vão apresentando uma estrutura mais oscilatória na vertical. Os modos verticais com m $>0$ são também referidos como modos internos.

Os resultados obtidos acima com relação às auto-soluções da equação da estrutura vertical são dependentes da suposição de uma estabilidade estática $\bar{\sigma}$ constante com a pressão. Outros trabalhos (Jacobs e Wiin-Nielsen 1966; Fulton e Schubert 1985; DeMaria 1985) obtiveram diferentes formas para as autofunções $G_{m}(p)$ no caso de um parâmetro de estabilidade estática $\bar{\sigma}$ mais realista. Jacobs e Wiin-Nielsen (1966) mostram que, para $\bar{\sigma}$ 
calculado para uma atmosfera com uma taxa de variação vertical de temperatura (TVVT) constante, o problema dado por (2.31) com as condições de fronteira (2.33) pode ser resolvido em termos de funções de Bessel. Wiin-Nielsen (1971) mostra que, para um estado básico isotérmico, o espectro vertical é constituído por uma parte discreta, dada pelo modo externo, e uma parte continua. DeMaria (1985) utilizou para a determinação dos modos verticais um perfil $\overline{\mathrm{T}}(\mathrm{p})$ representativo da atmosfera tropical sobre a América do Sul obtido a partir dos dados do FGGE III-b (First GARP Global Experiment). Para tais cálculos utilizando perfis realistas de temperatura, os autovalores e as autofunções $G_{m}(p)$ são geralmente obtidos a partir da solução da equação (2.31) por diferenças finitas, usando a condição de ortogonalidade (ver, por exemplo, Kasahara e Puri 1981). No entanto, dado o caráter teórico do presente estudo, o uso de um parâmetro de estabilidade estática $\bar{\sigma}$ constante simplifica a análise matemática e, portanto, é suficiente para os propósitos deste trabalho, não havendo necessidade de adotar perfis mais realistas do estado básico como feito em outros trabalhos.

Dados os valores de $\mathrm{c}_{\mathrm{m}}, \mathrm{m}=0,1,2, \ldots$, obtidos a partir dos autovalores $\lambda_{\mathrm{m}}$ da equação da estrutura vertical, a estrutura horizontal da solução do problema de $\mathrm{O}(1)$ é governada pelo sistema de equações (2.30), sujeito às condições de contorno periódicas em x e de decaimento conforme y $\rightarrow \pm \infty$. Este problema foi resolvido por Matsuno (1966) e as auto-soluções dessas equações correspondem às ondas equatoriais de Rossby, Poincaré (ondas de gravidadeinerciais), Kelvin e Yanai (ondas mistas de Rossby-gravidade). Uma discussão profunda a respeito da obtenção dessas soluções bem como acerca das propriedades dessas ondas pode ser encontrada em Silva Dias e Schubert (1979) e em Raupp (2002). No presente capítulo desta tese será apresentada uma breve revisão da obtenção dessas ondas a partir do sistema de equações dado por (2.30) e serão também discutidas algumas propriedades dessas soluções que são importantes para a compreensão dos resultados apresentados no Capítulo 5.

Supondo soluções na forma de ondas para o sistema (2.30), ou seja, $[\hat{u}(x, y, t, \tau), \hat{\mathrm{v}}(x, y, t, \tau), \hat{\phi}(x, y, t, \tau)]^{T}=A(\tau)\left[\hat{u}_{k}(y), \hat{\mathrm{v}}_{\mathrm{k}}(y), \hat{\phi}_{k}(y)\right]^{T} e^{i k x+i \varpi_{k} t}$, onde o sobre-índice "T" indica a transposição, $\mathrm{k}$ indica o número de onda zonal e $\varpi_{\mathrm{k}}$ a freqüência de oscilação, obtém-se

$$
\begin{array}{r}
i \varpi_{k} \hat{u}_{k}-y \hat{\mathrm{v}}_{\mathrm{k}}+i k \hat{\phi}_{k}=0 \\
i \varpi_{k} \hat{\mathrm{v}}_{\mathrm{k}}+y \hat{u}_{k}+\frac{d \hat{\phi}_{k}}{d y}=0
\end{array}
$$




$$
i \varpi_{k} \hat{\phi}_{k}+c_{m}^{2}\left(i k \hat{u}_{k}+\frac{d \hat{\mathrm{v}}_{\mathrm{k}}}{\mathrm{dy}}\right)=0
$$

O método utilizado por Matsuno (1966) para obter a solução do problema de autovalor/autovetor dado por (2.41) consiste em reduzir o sistema acima a uma única equação diferencial ordinária para a componente meridional do vento. De fato, após alguma álgebra é possível eliminar $\hat{u}_{\mathrm{k}}$ e $\phi_{\mathrm{k}}$ em (2.41) e obter a seguinte equação para $\mathrm{v}_{\mathrm{k}}$ :

$$
\frac{d^{2} \hat{\mathrm{v}}_{k}}{d y^{2}}+\left(\frac{\varpi_{k}^{2}}{c_{m}^{2}}-k^{2}+\frac{k}{\varpi_{k}}-\frac{y^{2}}{c_{m}^{2}}\right) \hat{\mathrm{v}}_{k}=0
$$

A solução da equação (2.42) somente satisfaz à condição de fronteira $\lim _{\mathrm{y} \rightarrow \pm \infty} \hat{\mathrm{v}}_{\mathrm{k}}=0$ se a auto-freqüência e o número de onda satisfizerem à seguinte relação de dispersão:

$$
\frac{\varpi_{m, k, n, r}^{2}}{c_{m}{ }^{2}}-k^{2}+\frac{k}{\varpi_{m, k, n, r}}=(2 n+1) \frac{1}{c_{m}} \quad, \mathrm{n}=0,1,2, \ldots
$$

Neste caso, a solução de (2.42) é dada por

$$
\hat{\mathrm{v}}_{\mathrm{m}, \mathrm{k}, \mathrm{n}, \mathrm{r}}(y)=H_{n}\left(\frac{y}{\sqrt{c_{m}}}\right) e^{-\frac{y^{2}}{2 c_{m}}}
$$

onde $\mathrm{H}_{\mathrm{n}}(\mathrm{y})$ representa o polinômio de Hermite de grau n. O índice $\mathrm{r}$ em (2.43) e em (2.44) indica o tipo de onda, pois para cada $\mathrm{k}, \mathrm{c}_{\mathrm{m}} \mathrm{e} \mathrm{n}$ fixados, a equação (2.43) constitui uma equação algébrica de terceiro grau em $\varpi_{\mathrm{m}, \mathrm{k}, \mathrm{n}, \mathrm{r}}$, cujas raízes são reais e indicam três tipos básicos de ondas: duas de alta freqüência, sendo uma se propagando para oeste e a outra para leste, e uma de baixa freqüência. Como pode ser notado em (2.41), esta última onda é caracterizada por um balanço geostrófico aproximado, enquanto as duas primeiras são caracterizadas por uma relação completamente ageostrófica entre os campos do vento e geopotencial, apresentando uma divergência significativa quando comparada com a da onda de baixa freqüência. A onda de baixa freqüência, ou quase-geostrófica, corresponde à onda de Rossby, enquanto as duas ondas de alta freqüência referem-se às ondas de gravidade-inerciais ou 
também conhecidas como ondas de Poincaré. Daqui a diante adotar-se-á a seguinte convenção para caracterizar essas ondas:

$$
\begin{aligned}
& \varpi_{\mathrm{m}, \mathrm{k}, \mathrm{n}, 1} \rightarrow \text { ondas de Rossby } \\
& \varpi_{\mathrm{m}, \mathrm{k}, \mathrm{n}, 2} \rightarrow \text { ondas de gravidade-inerciais com propagação para oeste } \\
& \varpi_{\mathrm{m},-\mathrm{k}, \mathrm{n}, 3} \rightarrow \text { ondas de gravidade-inerciais com propagação para leste. }
\end{aligned}
$$

Para o caso $n=0$ a equação (2.43) fornece apenas dois tipos de solução: uma onda de gravidade-inercial que se propaga para leste e um outro tipo de onda que se propaga para oeste e apresenta um comportamento de onda do tipo Rossby para k grande (isto é, $\varpi_{\mathrm{m}, \mathrm{k}, \mathrm{n}, \mathrm{r}}$ pequena e, portanto, quase-geostrófica) e do tipo gravidade-inercial para $\mathrm{k}$ pequeno (alta freqüência). Tal solução é denominada onda mista de Rossby-gravidade ou também conhecida como onda de Yanai. As autofunções de (2.41) são dadas, a partir de (2.44), na forma vetorial por:

$$
\xi_{a}(y)=\left[\begin{array}{c}
u_{a}(y) \\
\mathrm{v}_{\mathrm{a}}(y) \\
\phi_{a}(y)
\end{array}\right]=\left[\begin{array}{ccc}
c_{a} & 0 & 0 \\
0 & c_{a} & 0 \\
0 & 0 & c_{a}^{2}
\end{array}\right]\left[\begin{array}{c}
\frac{1}{2}\left(\widetilde{\varpi}_{\mathrm{a}}-\widetilde{\mathrm{k}}_{\mathrm{a}}\right) \mathrm{H}_{\mathrm{n}+1}\left(\frac{\mathrm{y}}{\sqrt{\mathrm{c}_{\mathrm{a}}}}\right)+\mathrm{n}\left(\widetilde{\varpi}_{\mathrm{a}}+\widetilde{\mathrm{k}}_{\mathrm{a}}\right) \mathrm{H}_{\mathrm{n}-1}\left(\frac{\mathrm{y}}{\sqrt{\mathrm{c}_{\mathrm{a}}}}\right) \\
i\left(\widetilde{\varpi}_{\mathrm{a}}{ }^{2}-\widetilde{\mathrm{k}}_{\mathrm{a}}^{2}\right) \mathrm{H}_{\mathrm{n}}\left(\frac{\mathrm{y}}{\sqrt{\mathrm{c}_{\mathrm{a}}}}\right) \\
\left.\frac{1}{2}\left(\widetilde{\varpi}_{\mathrm{a}}-\widetilde{\mathrm{k}}_{\mathrm{a}}\right) \mathrm{H}_{\mathrm{n}+1}\left(\frac{\mathrm{y}}{\sqrt{\mathrm{c}_{\mathrm{a}}}}\right)-\mathrm{n}\left(\widetilde{\varpi}_{\mathrm{a}}+\widetilde{\mathrm{k}}_{\mathrm{a}}\right) \mathrm{H}_{\mathrm{n}-1}\left(\frac{\mathrm{y}}{\sqrt{\mathrm{c}_{\mathrm{a}}}}\right)\right]
\end{array}\right] \frac{e^{-\frac{y^{2}}{2 c_{a}}}}{\left\|\xi_{a}\right\|}
$$

onde se utilizou em (2.45), e utilizar-se-á daqui a diante, uma notação condensada dos índices espectrais. Neste caso, o sub-índice a distingue um modo em particular, caracterizado pelo modo vertical $\mathrm{m}$, pelo número de onda zonal $\mathrm{k}$, pelo modo meridional $\mathrm{n}$ e pelo tipo de onda, representado por $r$, ou seja, $a=(m, k, n, r)$. É importante observar que o modo meridional $n$ distingue a estrutura meridional das autofunções $\xi_{\mathrm{a}}$, sendo as autofunções com n menores mais confinadas equatorialmente e, conforme $\mathrm{n}$ aumenta, os modos apresentam uma estrutura mais oscilatória em y, exibindo um caráter mais extratropical. Em (2.45) $\left\|\xi_{\mathrm{a}}\right\|$ refere-se à norma da autofunção $\xi_{\mathrm{a}}$, dada por 


$$
\left\|\xi_{a}\right\|=\left(\left\langle\xi_{a} \bullet \xi_{a}^{*}\right\rangle\right)^{\frac{1}{2}}
$$

onde o produto interno $<\bullet>$ é definido por

$$
\langle\vec{f} \bullet \vec{g}\rangle=\int_{-\infty}^{\infty}\left[f_{1} g_{1}^{*}+f_{2} g_{2}^{*}+f_{3} g_{3}^{*}\right] d y
$$

com $\vec{f}$ e $\vec{g}$ representando funções vetoriais quaisquer que satisfazem às condições de contorno em y dadas por (2.10b); os sub-índices 1,2 e 3 referem-se às suas respectivas componentes escalares e o sobre-índice * indica o complexo conjugado. Substituindo (2.45) em (2.46) é possível mostrar que $\left\|\xi_{\mathrm{a}}\right\|$ é dada pela seguinte expressão:

$$
\left\|\xi_{\mathrm{a}}\right\|=\left\{2^{\mathrm{n}} \mathrm{n} ! \pi^{1 / 2}\left[\left(\widetilde{\varpi}_{a}-\widetilde{k}\right)^{2}(\mathrm{n}+1)+\mathrm{n}\left(\widetilde{\varpi}_{a}+\widetilde{k}\right)^{2}+\left(\widetilde{\varpi}_{a}{ }^{2}-\widetilde{k}^{2}\right)^{2}\right]\right\}^{1 / 2}
$$

Nas equações (2.45) e (2.48), os parâmetros $\widetilde{\varpi}_{a}$ e $\widetilde{k}$ são relacionados com as autofreqüências e o número de onda zonal através das seguintes expressões:

$$
\widetilde{\varpi}_{a}=\frac{\varpi_{a}}{\sqrt{c_{a}}} \quad, \quad \tilde{k}=k \sqrt{c_{a}}
$$

O sistema (2.41) também admite um tipo especial de onda, não incluída em (2.42), que apresenta a componente meridional do vento identicamente nula. Tal solução refere-se à onda de Kelvin e é caracterizada pela relação de dispersão $\varpi_{\mathrm{a}}=-\mathrm{kc}_{\mathrm{a}}$ e, portanto, se propaga para leste de forma não dispersiva. Uma vez que esta relação de dispersão pode ser obtida a partir de (2.43) para $n=-1$, Matsuno (1966) rotulou tal solução como $n=-1$. Observa-se a partir das equações (2.41) para $\hat{\mathrm{v}}_{\mathrm{k}}=0$ que esta onda é caracterizada por um balanço geostrófico entre a componente zonal do vento e o gradiente meridional de pressão, enquanto na direção zonal as perturbações do vento associadas a esta onda são totalmente divergentes (equações 2.41a e 2.41c). Resolvendo o sistema algébrico formado pelas equações (2.41a) e (2.41c) para $\hat{\mathrm{v}}_{\mathrm{k}}=0$ segue que para o modo de Kelvin tem-se que $\mathrm{u}_{\mathrm{a}}=\phi_{\mathrm{a}}=D e^{-\frac{y^{2}}{2 c_{c}}}$, sendo D uma constante arbitrária. Assim, uma expressão apropriada para as autofunções $\xi_{\text {a }}$ para $n=-1$ pode ser dada por 


$$
\xi_{a}(y)=\left[\begin{array}{ccc}
c_{a} & 0 & 0 \\
0 & c_{a} & 0 \\
0 & 0 & c_{a}{ }^{2}
\end{array}\right]\left[\begin{array}{l}
1 \\
0 \\
1
\end{array}\right] \frac{\mathrm{e}^{-\frac{y^{2}}{2 c_{a}}}}{(2 \sqrt{\pi})^{\frac{1}{2}}} \quad, \quad \text { para } \quad \mathrm{n}=-1
$$

É interessante observar a partir da expressão (2.50) acima que as perturbações induzidas pelas ondas de Kelvin são estritamente confinadas na região equatorial. O sistema (2.41) também possui um autovalor degenerado representado por $\varpi_{\mathrm{m}, 0, \mathrm{n}, 1}=0$. As autofunções correspondentes a este autovalor degenerado são caracterizadas por uma estrutura zonalmente simétrica $(\mathrm{k}=0)$, absolutamente geostrófica e com a componente meridional do vento identicamente nula. Estas autofunções para estes modos geostróficos zonalmente simétricos podem ser obtidas através do limite assintótico da expressão (2.45) para as ondas de Rossby quando $\mathrm{k} \rightarrow$ 0, conforme mostrado por Silva Dias e Schubert (1979). A expressão final é dada por

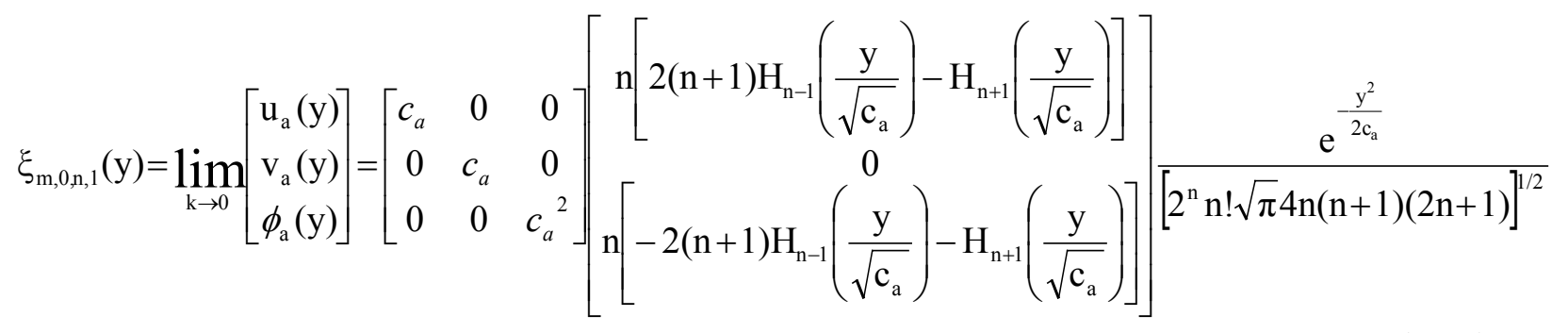

As autofunções $\xi_{\mathrm{a}}(\mathrm{y})$ dadas por (2.45), (2.50) e (2.51) formam um conjunto ortogonal e completo no espaço das funções de quadrado integrável no intervalo $(-\infty,+\infty)$ (Matsuno 1966; Silva Dias e Schubert 1979).

Um aspecto interessante e bastante importante para os propósitos do presente trabalho acerca das autofunções $\xi_{\mathrm{a}}$ refere-se à dependência do grau de confinamento equatorial com relação à constante de separação $c_{a}$. A partir das equações (2.45) e (2.51) é facilmente notável que quanto maior o valor de $c_{a}$ menor é o grau de confinamento equatorial desses modos. De fato, a partir da equação (2.42) segue que as autofunções apresentam uma estrutura oscilatória no intervalo dado por $|\mathrm{y}|<\left|\mathrm{y}_{\mathrm{c}}\right|$, passando a decrescer exponencialmente à medida que se afasta do equador se $|\mathrm{y}|>\left|\mathrm{y}_{\mathrm{c}}\right|$, de modo a satisfazer à condição de fronteira; sendo $\mathrm{y}_{\mathrm{c}}$ a latitude crítica que representa a distância a partir do equador na qual o índice de refração é nulo e, por 
conseguinte, a solução muda de um comportamento oscilatório para um comportamento evanescente. A partir de (2.42) pode ser facilmente notado que esta latitude crítica é dada por

$$
y_{c}= \pm \sqrt{c_{a}{ }^{2}\left(\frac{\varpi_{a}^{2}}{c_{a}{ }^{2}}-k^{2}+\frac{k}{\varpi_{a}}\right)}= \pm \sqrt{(2 n+1) c_{a}}
$$

Logo, usando os valores de $c_{\mathrm{a}}$ mostrados na Tabela 2.1 e considerando a escala de comprimento característica $\mathrm{L}=1000 \mathrm{Km}$, segue que o valor de $\mathrm{y}_{\mathrm{c}}$ para o primeiro modo baroclínico corresponde a uma latitude de aproximadamente $20^{\circ}$ e $26^{\circ}$ para $n=1$ e $n=2$, respectivamente. Em contrapartida, o valor de $y_{c}$ para o modo externo corresponde a uma latitude de aproximadamente $58^{\circ}$ e $75^{\circ}$ para $n=1$ e $n=2$, respectivamente. Dessa forma, é claramente notável a partir desses valores que as perturbações decorrentes da atividade das ondas associadas aos modos baroclínicos somente apresentam amplitudes significativas dentro da faixa tropical, ou seja, são bastante confinadas equatorialmente. Em contrapartida, as perturbações associadas às ondas barotrópicas apresentam uma significativa influência em regiões de latitudes médias e altas. De fato, vários estudos observacionais destacam que modos de variabilidade atmosférica característicos da região equatorial, como por exemplo as perturbações na circulação atmosférica induzidas pela oscilação de Madden-Julian (OMJ), que corresponde ao modo dominante da variabilidade atmosférica intra-sazonal, apresentam uma estrutura vertical similar à correspondente ao modo vertical $\mathrm{m}=1$ mostrada na Fig. 2.2b. Da mesma maneira, estudos observacionais tais como os de Wallace e Gutzler (1981), Blackmon et. al. (1984) e outros destacam que os padrões de teleconexões envolvendo regiões de latitudes médias e altas, tais como o padrão do Pacífico/América do Norte (PNA), apresentam uma estrutura barotrópica equivalente. A dominância do modo externo na representação da energética do escoamento atmosférico em latitudes médias e altas também é evidenciada por Silva Dias e Bonatti (1985). Como será destacado no Capítulo 4, é possível a existência de tríades ressonantes envolvendo ondas de Rossby externas e ondas equatoriais internas de Kelvin e de Yanai e que a dinâmica de tais interações abordadas no Capítulo 5 tem implicações importantes com relação à interação entre as circulações atmosféricas tropical e extratropical.

Dessa forma, a solução do problema de $\mathrm{O}(1)$ pode ser escrita da seguinte forma: 


$$
\left[\begin{array}{l}
u^{(0)}(x, y, p, t, \tau) \\
\mathrm{v}^{(0)}(x, y, p, t, \tau) \\
\phi^{(0)}(x, y, p, t, \tau)
\end{array}\right]=\sum_{a} A_{a}(\tau) \xi_{a}(y) e^{i k_{a} x+i \varpi_{a} t} G_{a}(p)
$$

sendo que, como já mencionado anteriormente, o sub-índice a caracteriza um modo em particular e é dado por a $=(\mathrm{m}, \mathrm{k}, \mathrm{n}, \mathrm{r})$, com $\mathrm{m}$ caracterizando a estrutura vertical, $\mathrm{k}$ a estrutura zonal, $\mathrm{n}$ a estrutura meridional e $\mathrm{r}$ o tipo de onda associado. A amplitude dessas ondas, representada por $\mathrm{A}_{\mathrm{a}}$, varia somente na escala longa de tempo e esta evolução será determinada a partir da condição de solvabilidade do problema de $\mathrm{O}(\varepsilon)$, como será mostrado mais adiante. Entretanto, como será visto mais adiante, para tanto é necessário não somente conhecer a solução do problema de $\mathrm{O}(1)$, representada por $\mathrm{u}^{(0)}, \mathrm{v}^{(0)}$ e $\phi^{(0)}$ dada por (2.53) acima, como também conhecer a solução adjunta, ou seja, a solução do problema adjunto ao problema de $\mathrm{O}(1)$ dado por (2.25).

\subsubsection{O Problema de $\mathrm{O}(1)$ Adjunto}

O problema de $\mathrm{O}(1)$ representado por (2.25) pode também ser escrito na forma vetorial da seguinte forma:

$$
\mathfrak{I} \xi^{(0)}=0
$$

onde $\xi^{(0)}=\left[\mathrm{u}^{(0)}, \mathrm{v}^{(0)}, \phi^{(0)}\right]^{\mathrm{T}}$ representa o vetor solução e $\mathfrak{I}$ é o operador linear, dado por

$$
\mathfrak{I}=\left[\begin{array}{ccc}
\frac{\partial}{\partial t} & -y & \frac{\partial}{\partial x} \\
y & \frac{\partial}{\partial t} & \frac{\partial}{\partial y} \\
-\frac{\partial}{\partial x} & -\frac{\partial}{\partial y} & \frac{\partial}{\partial t} \frac{\partial}{\partial p}\left(\frac{1}{\tilde{\widetilde{\sigma}}} \frac{\partial}{\partial p}\right)
\end{array}\right]
$$

Assim, o operador adjunto de $\mathfrak{I}$ é definido como sendo o operador $\mathfrak{I}^{+}$que satisfaz à seguinte condição 
$\lim _{\chi \rightarrow \infty} \frac{1}{\chi 2 \widetilde{L}_{x}} \int_{0}^{\chi} \int_{-\widetilde{L}_{x}}^{\widetilde{L}_{x}} \int_{\widetilde{p}_{T}}^{1}\langle\vec{J} \vec{P} \bullet \vec{Q}\rangle d p d x d t=\lim _{\chi \rightarrow \infty} \frac{1}{\chi 2 \widetilde{L}_{x}} \int_{0}^{\chi} \int_{-\widetilde{L}_{x}}^{\widetilde{L}_{x}} \int_{\widetilde{p}_{T}}^{1}\left\langle\vec{P} \bullet \mathfrak{J}^{+} \vec{Q}\right\rangle d p d x d t$

para quaisquer funções vetoriais $\vec{P}$ e $\vec{Q}$ que sejam de quadrado integrável em $[0, \infty) \mathrm{X}$ $\left[-\widetilde{L}_{x}, \widetilde{L}_{x}\right] \mathrm{X}(-\infty, \infty) \mathrm{X}\left[\widetilde{p}_{T}, 1\right]$. Na condição acima, $\widetilde{L}_{x}=\frac{L_{x}}{L}$ representa o perímetro equatorial da Terra na escala adimensional e $<\bullet>$ representa o produto interno em y definido de acordo com (2.47). Da integração por partes de (2.56) segue que o operador $\mathfrak{J}^{+}$é dado por

$$
\mathfrak{I}^{+}=\left[\begin{array}{ccc}
-\frac{\partial}{\partial t} & y & \frac{\partial}{\partial x} \\
-y & -\frac{\partial}{\partial t} & \frac{\partial}{\partial y} \\
-\frac{\partial}{\partial x} & -\frac{\partial}{\partial y} & -\frac{\partial}{\partial t} \frac{\partial}{\partial p}\left(\frac{1}{\widetilde{\widetilde{\sigma}}} \frac{\partial}{\partial p}\right)
\end{array}\right]
$$

Logo, o problema de $\mathrm{O}(1)$ adjunto é escrito como

$$
\begin{aligned}
& -\frac{\partial u^{(0)^{+}}}{\partial t}+y \mathrm{v}^{(0)^{+}}+\frac{\partial \phi^{(0)^{+}}}{\partial x}=0 \\
& -\frac{\partial \mathrm{v}^{(0)^{+}}}{\partial t}-y \mathrm{u}^{(0)^{+}}+\frac{\partial \phi^{(0)^{+}}}{\partial y}=0 \\
& -\frac{\partial}{\partial t} \frac{\partial}{\partial p}\left(\frac{1}{\widetilde{\widetilde{\sigma}}} \frac{\partial \phi^{(0)^{+}}}{\partial p}\right)-\left(\frac{\partial u^{(0)^{+}}}{\partial x}+\frac{\partial \mathrm{v}^{(0)^{+}}}{\partial y}\right)=0
\end{aligned}
$$

Da mesma maneira, da integração por partes de (2.56) também resulta que as condições de fronteira do problema de $\mathrm{O}(1)$ adjunto são dadas por

$$
\lim _{y \rightarrow \pm \infty}\left[\begin{array}{l}
u^{(0)+} \\
\mathrm{v}^{(0)+} \\
\phi^{(0)+}
\end{array}\right](x, y, p, t, \tau)=0
$$




$$
\frac{\partial}{\partial p} \frac{\partial \phi^{(0)^{+}}}{\partial t}+\widetilde{\bar{\rho}} \widetilde{\bar{\sigma}} \frac{\partial \phi^{(0)^{+}}}{\partial t}=0 \quad \text { em } \mathrm{p}=1 \text { e } \quad \text { em } \mathrm{p}=\widetilde{p}_{T}
$$

bem como periodicidade em x. Assim, usando uma separação de variáveis similar àquela do problema de $\mathrm{O}(1)$ obtém-se

$$
\begin{aligned}
& \left(-\frac{\partial \hat{u}^{+}}{\partial t}+y \hat{\mathrm{v}}^{+}+\frac{\partial \hat{\phi}^{+}}{\partial x}\right) G^{+}=0 \\
& \left(-\frac{\partial \hat{\mathrm{v}}^{+}}{\partial t}-y \hat{\mathrm{u}}^{+}+\frac{\partial \hat{\phi}^{+}}{\partial y}\right) G^{+}=0 \\
& -\frac{\partial \hat{\phi}^{+}}{\partial t} \frac{d}{d p}\left(\frac{1}{\widetilde{\widetilde{\sigma}}} \frac{d G^{+}}{d p}\right)-\left(\frac{\partial \hat{\mathrm{u}}^{+}}{\partial x}+\frac{\partial \hat{\mathrm{v}}^{+}}{\partial y}\right) G^{+}=0
\end{aligned}
$$

De $(2.59 b)$ resulta que as condições de fronteira na vertical para as autofunções $\mathrm{G}^{+}(\mathrm{p})$ são as mesmas do problema de $\mathrm{O}(1)$ original dadas por (2.33). Com isso, segue de (2.60c) que

$$
\frac{-\frac{\partial \hat{\phi}^{+}}{\partial t}}{\left(\frac{\partial \hat{u}^{+}}{\partial x}+\frac{\partial \hat{\mathrm{v}}^{+}}{\partial y}\right)}=\frac{G^{+}}{\frac{d}{d p}\left(\frac{1}{\widetilde{\widetilde{\sigma}}} \frac{d G^{+}}{d p}\right)}=-c^{2}
$$

onde o sinal negativo na constante de separação no lado direito de (2.61) é necessário para que a solução satisfaça às condições de contorno na vertical. Assim, de (2.61) resulta que a equação da estrutura vertical do problema adjunto é exatamente a mesma do problema de $\mathrm{O}(1)$ original dada por (2.31) e, conseqüentemente, as autofunções na vertical também são as mesmas do problema original, ou seja, $\mathrm{G}^{+}(\mathrm{p})=\mathrm{G}(\mathrm{p})$. Como resultado, a estrutura horizontal do problema adjunto é governada pelo seguinte sistema:

$$
\begin{aligned}
& -\frac{\partial \hat{u}^{+}}{\partial t}+y \hat{\mathbf{v}}^{+}+\frac{\partial \hat{\phi}^{+}}{\partial x}=0 \\
& -\frac{\partial \hat{\mathrm{v}}^{+}}{\partial t}-y \hat{\mathrm{u}}^{+}+\frac{\partial \hat{\phi}^{+}}{\partial y}=0
\end{aligned}
$$




$$
-\frac{\partial \hat{\phi}^{+}}{\partial t}+c^{2}\left(\frac{\partial \hat{u}^{+}}{\partial x}+\frac{\partial \hat{\mathrm{v}}^{+}}{\partial y}\right)=0
$$

Fazendo a seguinte transformação das variáveis independentes $\mathrm{t} \rightarrow-\mathrm{t}, \mathrm{x} \rightarrow-\mathrm{x}$ e $\mathrm{y} \rightarrow-\mathrm{y}$ e substituindo-a nas equações (2.62), obtém-se

$$
\begin{aligned}
& \frac{\partial \hat{u}^{+}}{\partial t}-y \hat{\mathrm{v}}^{+}-\frac{\partial \hat{\phi}^{+}}{\partial x}=0 \\
& \frac{\partial \hat{\mathrm{v}}^{+}}{\partial t}+y \hat{\mathrm{u}}^{+}-\frac{\partial \hat{\phi}^{+}}{\partial y}=0 \\
& -\frac{\partial \hat{\phi}^{+}}{\partial t}+c^{2}\left(\frac{\partial \hat{u}^{+}}{\partial x}+\frac{\partial \hat{\mathrm{v}}^{+}}{\partial y}\right)=0
\end{aligned}
$$

Dessa forma, comparando as equações (2.63) acima com as equações (2.30) é fácil verificar que as autofunções da estrutura horizontal do operador $\mathfrak{I}^{+}$são dadas por

$$
\xi_{a}^{+}(y)=\left[\begin{array}{c}
u_{a}(-y) \\
\mathrm{v}_{\mathrm{a}}(-y) \\
-\phi_{a}(-y)
\end{array}\right]
$$

onde $u_{a}, v_{a}$ e $\phi_{a}$ representam as autofunções do problema original, dadas por (2.45), (2.50) e (2.51). Entretanto, usando as propriedades de paridade e imparidade das funções de Hermite em função do modo meridional n é possível obter uma expressão ainda mais simplificada para as autofunções $\xi_{\mathrm{a}}{ }^{+}$. Observando a equação (2.45) é possível verificar que se o modo meridional $n$ é par, as componentes $u_{a}$ e $\phi_{a}$ são anti-simétricas em relação ao equador e a componente $\mathrm{v}_{\mathrm{a}}$ é simétrica; por outro lado, se $\mathrm{n}$ é ímpar, $\mathrm{u}_{\mathrm{a}}$ e $\phi_{\mathrm{a}}$ são funções pares e $\mathrm{v}_{\mathrm{a}}$ é uma função ímpar. Conseqüentemente, as autofunções da estrutura meridional do problema adjunto podem também ser escritas da seguinte forma:

$$
\xi_{a}^{+}(y)=\left[\begin{array}{c}
u_{a}(y) \\
\mathrm{v}_{\mathrm{a}}^{*}(y) \\
-\phi_{a}(y)
\end{array}\right]
$$


onde o sobre-índice * representa o complexo conjugado. Assim, a solução do problema de $\mathrm{O}(1)$ adjunto é dada por

$$
\left[\begin{array}{l}
u^{(0)^{+}}(x, y, p, t, \tau) \\
\mathrm{v}^{(0)^{+}}(x, y, p, t, \tau) \\
\phi^{(0)^{+}}(x, y, p, t, \tau)
\end{array}\right]=\sum_{a} A_{a}(\tau) \xi_{a}^{+}(y) e^{-i k_{a} x-i \omega_{a} t} G_{a}(p)
$$

\subsubsection{O Problema de $\mathrm{O}(\varepsilon)$}

O sistema de equações (2.23) para a $\mathrm{O}(\varepsilon)$ é dado por

$$
\begin{aligned}
& \frac{\partial u^{(1)}}{\partial t}-y \mathrm{v}^{(1)}+\frac{\partial \phi^{(1)}}{\partial x}=-\frac{\partial u^{(0)}}{\partial \tau}-u^{(0)} \frac{\partial u^{(0)}}{\partial x}-\mathrm{v}^{(0)} \frac{\partial u^{(0)}}{\partial y}-F \omega^{(0)} \frac{\partial u^{(0)}}{\partial p} \\
& \frac{\partial \mathrm{v}^{(1)}}{\partial t}+y \mathrm{u}^{(1)}+\frac{\partial \phi^{(1)}}{\partial y}=-\frac{\partial \mathrm{v}^{(0)}}{\partial \tau}-u^{(0)} \frac{\partial \mathrm{v}^{(0)}}{\partial x}-\mathrm{v}^{(0)} \frac{\partial \mathrm{v}^{(0)}}{\partial y}-F \omega^{(0)} \frac{\partial \mathrm{v}^{(0)}}{\partial p} \\
& \frac{\partial}{\partial t} \frac{\partial}{\partial p}\left(\frac{1}{\widetilde{\widetilde{\sigma}}} \frac{\partial \phi^{(1)}}{\partial p}\right)-\left(\frac{\partial u^{(1)}}{\partial x}+\frac{\partial \mathrm{v}^{(1)}}{\partial y}\right)=-\frac{\partial}{\partial \tau} \frac{\partial}{\partial p}\left(\frac{1}{\widetilde{\widetilde{\sigma}}} \frac{\partial \phi^{(0)}}{\partial p}\right)- \\
& \frac{\partial}{\partial p}\left[\frac{u^{(0)}}{\widetilde{\widetilde{\sigma}}} \frac{\partial}{\partial x} \frac{\partial \phi^{(0)}}{\partial p}+\frac{\mathrm{v}^{(0)}}{\widetilde{\widetilde{\sigma}}} \frac{\partial}{\partial y} \frac{\partial \phi^{(0)}}{\partial p}+\frac{F \omega^{(0)}}{\widetilde{\widetilde{\sigma}}} \frac{\partial^{2} \phi^{(0)}}{\partial p^{2}}+\frac{F \omega^{(0)}}{\widetilde{\sigma} p}(1-\kappa) \frac{\partial \phi^{(0)}}{\partial p}\right]
\end{aligned}
$$

Na forma vetorial, (2.67) pode ser escrito como:

$$
\mathfrak{I} \xi^{(1)}=N^{(0)}
$$

onde $\mathfrak{I}$ é o operador linear dado por $(2.55), \xi^{(1)}=\left[\mathrm{u}^{(1)}, \mathrm{v}^{(1)}, \phi^{(1)}\right]^{\mathrm{T}}$ representa o vetor solução do problema de $\mathrm{O}(\varepsilon)$ e $\mathrm{N}^{(0)}$ é o vetor que contém a evolução na escala longa de tempo da solução do problema de $\mathrm{O}(1)$ e a contribuição de ordem dominante dos termos não lineares, sendo dado por 


$$
N^{(0)}=\left[\begin{array}{c}
-\frac{\partial u^{(0)}}{\partial \tau}-u^{(0)} \frac{\partial u^{(0)}}{\partial x}-\mathrm{v}^{(0)} \frac{\partial u^{(0)}}{\partial y}-F \omega^{(0)} \frac{\partial u^{(0)}}{\partial p} \\
-\frac{\partial \mathrm{v}^{(0)}}{\partial \tau}-u^{(0)} \frac{\partial \mathrm{v}^{(0)}}{\partial x}-\mathrm{v}^{(0)} \frac{\partial \mathrm{v}^{(0)}}{\partial y}-F \omega^{(0)} \frac{\partial \mathrm{v}^{(0)}}{\partial p} \\
-\frac{\partial}{\partial \tau} \frac{\partial}{\partial p}\left(\frac{1}{\widetilde{\widetilde{\sigma}}} \frac{\partial \phi^{(0)}}{\partial p}\right)-\frac{\partial}{\partial p}\left[\frac{u^{(0)}}{\widetilde{\sigma}} \frac{\partial}{\partial x} \frac{\partial \phi^{(0)}}{\partial p}+\frac{\mathrm{v}^{(0)}}{\widetilde{\sigma}} \frac{\partial}{\partial y} \frac{\partial \phi^{(0)}}{\partial p}+\frac{F \omega^{(0)}}{\widetilde{\widetilde{\sigma}}} \frac{\partial^{2} \phi^{(0)}}{\partial p^{2}}+\frac{F \omega^{(0)}}{\widetilde{\sigma} p}(1-\kappa) \frac{\partial \phi^{(0)}}{\partial p}\right]
\end{array}\right]
$$

Similarmente, as condições de fronteira verticais (2.24) para a $\mathrm{O}(\varepsilon)$ são dadas por

$$
\frac{\partial \phi^{(1)}}{\partial t}+\frac{\partial \phi^{(0)}}{\partial \tau}+u^{(0)} \frac{\partial \phi^{(0)}}{\partial x}+\mathrm{v}^{(0)} \frac{\partial \phi^{(0)}}{\partial y}+F \omega^{(0)} \frac{\partial \phi^{(0)}}{\partial p}-\frac{F \omega^{(1)}}{\widetilde{\bar{\rho}}_{0}}+\widetilde{\bar{\rho}}_{0} \phi^{(0)} \frac{\partial}{\partial p}\left[\frac{\partial \phi^{(0)}}{\partial t}-\frac{F \omega^{(0)}}{\widetilde{\bar{\rho}}}\right]=0
$$

em $p=1$

$$
\frac{\partial \phi^{(1)}}{\partial t}+\frac{\partial \phi^{(0)}}{\partial \tau}+u^{(0)} \frac{\partial \phi^{(0)}}{\partial x}+\mathrm{v}^{(0)} \frac{\partial \phi^{(0)}}{\partial y}+F \omega^{(0)} \frac{\partial \phi^{(0)}}{\partial p}-\frac{F \omega^{(1)}}{\widetilde{\bar{\rho}}_{T}}-\widetilde{\bar{\rho}}_{T} \phi^{(0)} \frac{\partial}{\partial p}\left[\frac{\partial \phi^{(0)}}{\partial t}-\frac{F \omega^{(0)}}{\widetilde{\bar{\rho}}}\right]=0
$$

em $\mathrm{p}=\tilde{p}_{T}$

Como mencionado anteriormente, as expansões assintóticas representadas por (2.22) constituem a hipótese básica do método de perturbação aplicado às equações (2.18), sujeitas às condições de fronteira (2.19). No entanto, para que estas expansões assintóticas sejam uniformemente válidas é necessário que os termos $\mathrm{u}^{(0)}, \mathrm{v}^{(0)}, \phi^{(0)}$ e $\omega^{(0)}$ representem, formalmente, os efeitos de ordem dominante. Em outras palavras, as expansões assintóticas representadas por (2.22) não fazem sentido se os termos $\varepsilon \mathrm{u}^{(1)}, \varepsilon \mathrm{v}^{(1)}, \varepsilon \phi^{(1)} \mathrm{e} \varepsilon \omega^{(1)}$ não forem, de fato, pequenos comparados com os respectivos termos $\mathrm{u}^{(0)}, \mathrm{v}^{(0)}, \phi^{(0)}$ e $\omega^{(0)}$. Por outro lado, para que os termos $\varepsilon u^{(1)}, \varepsilon v^{(1)}, \varepsilon \phi^{(1)}$ e $\varepsilon \omega^{(1)}$ sejam de fato pequenos em comparação com os respectivos termos $u^{(0)}, v^{(0)}, \phi^{(0)}$ e $\omega^{(0)}$ é necessário que os coeficientes $u^{(1)}, v^{(1)}, \phi^{(1)}$ e $\omega^{(1)}$ cresçam sub-linearmente na escala rápida de tempo t (Majda 2003), i.e.,

$$
\lim _{t \rightarrow \infty} \frac{\left|u^{(1)}, \mathrm{v}^{(1)}, \phi^{(1)}, \omega^{(1)}\right|}{|t|}=0
$$


De fato, se o coeficiente $u^{(1)}$, por exemplo, crescer linearmente em t, i.e, se $u^{(1)} \sim|t|$ para $t$ suficientemente grande, o segundo termo da série representado por $\varepsilon^{(1)}$ neste caso seria dado por $\varepsilon \mathrm{u}^{(1)} \sim \varepsilon|\mathrm{t}|=|\tau|$. Logo, o segundo termo na expansão em série dada por (2.22) neste caso apresentaria, para t suficientemente grande, a mesma ordem de magnitude do primeiro termo $\mathrm{u}^{(0)}$. Assim, a condição de crescimento sub-linear em $\mathrm{t}$ para os termos $\mathrm{u}^{(1)}, \mathrm{v}^{(1)}, \phi^{(1)} \mathrm{e}$ $\omega^{(1)}$ é crucialmente necessária para a validade das expansões assintóticas dadas por (2.22).

Uma vez que a solução do problema de $\mathrm{O}(1)$ é conhecida, o problema (2.68), com as condições de fronteira verticais dadas por (2.70), constitui um problema linear e não homogêneo para $\left[\mathrm{u}^{(1)}, \mathrm{v}^{(1)}, \phi^{(1)}\right]^{\mathrm{T}}$. Além disso, é claramente notável nas equações (2.67)-(2.70) que a solução da parte homogênea deste sistema é idêntica à solução do problema de $\mathrm{O}(1)$ dada por (2.53), juntamente com (2.37), (2.45), (2.50) e (2.51), em sua dependência em $(\mathrm{x}, \mathrm{y}, \mathrm{p}, \mathrm{t})$. Conseqüentemente, analisando os termos não homogêneos em (2.67) e em (2.70) é possível verificar que para cada harmônico da solução $\xi^{(1)}$ existe uma correspondente forçante harmônica ressonante que surge dos termos relacionados com a evolução na escala longa de tempo da solução $\xi^{(0)}$. Os produtos não lineares em (2.67) e (2.70) também podem ser ressonantes com algum harmônico da solução $\xi^{(1)}$ sob determinadas condições, como será mostrado mais adiante. Logo, tais forçantes harmônicas ressonantes podem, potencialmente, gerar termos em $\xi^{(1)}$ que crescem linearmente com a escala rápida de tempo t. Estas soluções que crescem linearmente em t são comumente chamadas de soluções seculares, pois apresentam um crescimento ilimitado no tempo. Dessa forma, para garantir a condição de crescimento sub-linear para os coeficientes $u^{(1)}, v^{(1)}, \phi^{(1)}$ e $\omega^{(1)}$ e, conseqüentemente, garantir a validade das expansões assintóticas representadas por (2.22), é necessário que estas soluções seculares sejam nulas. Para tanto, é necessário que o termo forçante seja ortogonal às autofunções adjuntas referentes aos modos ressonantes à forçante (Majda 2003, Capítulo 5). Neste caso, o produto interno envolvendo o termo forçante e a autofunção do operador linear adjunto referente ao modo ressonante deve ser nulo. Assim, a condição de solvabilidade que garante que a solução $\xi^{(1)}$ em (2.68) seja limitada na escala rápida de tempo t é dada por: 


$$
\begin{aligned}
& \lim _{\chi \rightarrow \infty} \frac{1}{2 \chi \widetilde{L}_{x}} \int_{0}^{\chi} \int_{0}^{\widetilde{L}_{x}} \int_{\widetilde{L}_{x}}^{1}\left\langle\widetilde{T}_{T} \xi^{(1)} \bullet \xi_{a}^{+}(y) e^{-i k_{a} x-i \varpi_{a} t} G_{a}(p)\right\rangle d p d x d t= \\
& \lim _{\chi \rightarrow \infty} \frac{1}{2 \chi \widetilde{L}_{x}} \int_{0}^{\chi} \int_{-\widetilde{L}_{x}}^{\widetilde{L}_{x}} \int_{\widetilde{p}_{T}}^{1}\left\langle N^{(0)} \bullet \xi_{a}^{+}(y) e^{-i k_{a} x-i \varpi_{a} t} G_{a}(p)\right\rangle d p d x d t
\end{aligned}
$$

Assim como em (2.56), na equação (2.72) acima $\widetilde{L}_{x}=\frac{L_{x}}{L}$ representa o perímetro equatorial da Terra na escala adimensional $\mathrm{e}<\bullet<$ representa o produto interno em y definido de acordo com (2.47). Na equação (2.72) acima, $\xi_{a}^{+}(y) e^{-i k_{a} x-i \varpi_{a} t} G_{a}(p)$ representa uma dada autofunção do operador $\mathfrak{I}^{+}$. Desenvolvendo o produto interno no lado esquerdo de (2.72) de acordo com (2.47), chega-se à seguinte expressão:

$\lim _{\chi \rightarrow \infty} \frac{1}{2 \chi \widetilde{L}_{x}} \int_{-\widetilde{L}_{x}}^{\widetilde{L}_{x}} \int_{-\infty}^{+\infty} \int_{\tilde{p}_{T}}^{1} \int_{0}^{\chi} \frac{\partial u^{(1)}}{\partial t} u_{a}^{+}(y) e^{-i k_{a} x} e^{-i \sigma_{a} t} G_{a}(p) d t d p d y d x-$

$\lim _{\chi \rightarrow \infty} \frac{1}{2 \chi \widetilde{L}_{x}} \int_{-\widetilde{L}_{x}}^{\widetilde{L}_{x}} \int_{-\infty}^{+\infty} \int_{\tilde{p}_{T}}^{1} \int_{0}^{\chi} y \mathrm{v}^{(1)} u_{a}^{+}(y) e^{-i k_{a} x} e^{-i \omega_{a} t} G_{a}(p) d t d p d y d x+$

$\lim _{\chi \rightarrow \infty} \frac{1}{2 \chi \widetilde{L}_{x}} \int_{0}^{\chi} \int_{-\infty}^{+\infty} \int_{\tilde{L}_{T}-\widetilde{L}_{x}}^{1} \int_{\widetilde{L}_{x}}^{\widetilde{L}_{x}} \frac{\partial \phi^{(1)}}{\partial x} u_{a}^{+}(y) e^{-i k_{a} x} e^{-i \varpi_{a} t} G_{a}(p) d x d p d y d t+$

$\lim _{\chi \rightarrow \infty} \frac{1}{2 \chi \widetilde{L}_{x}} \int_{-\widetilde{L}_{x}}^{\widetilde{L}_{x}} \int_{-\infty}^{+\infty} \int_{\widetilde{p}_{T}}^{1} \int_{0}^{\chi} \frac{\partial \mathrm{v}^{(1)}}{\partial t} \mathrm{v}_{a}^{+}(y) e^{-i k_{a} x} e^{-i \sigma_{a} t} G_{a}(p) d t d p d y d x+$

$\lim _{\chi \rightarrow \infty} \frac{1}{2 \chi \widetilde{L}_{x}} \int_{-\widetilde{L}_{x}}^{\widetilde{L}_{x}} \int_{-\infty}^{+\infty} \int_{\widetilde{p}_{T}}^{1} \int_{0}^{\chi} \mathrm{yu}^{(1)} \mathrm{v}_{a}^{+}(y) e^{-i k_{a} x} e^{-i \varpi_{a} t} G_{a}(p) d t d p d y d x+$

$\lim _{\chi \rightarrow \infty} \frac{1}{2 \chi \widetilde{L}_{x}} \int_{-\widetilde{L}_{x}}^{\widetilde{L}_{x}} \int_{0}^{\chi} \int_{\tilde{p}_{T}-\infty}^{1} \int_{\tau^{-\infty}}^{+\infty} \frac{\partial \phi^{(1)}}{\partial y} \mathrm{v}_{a}^{+}(y) e^{-i k_{a} x} e^{-i \varpi_{a} t} G_{a}(p) d y d p d t d x-$

$\lim _{\chi \rightarrow \infty} \frac{1}{2 \chi \widetilde{L}_{x}} \int_{0}^{\chi} \int_{-\infty}^{+\infty} \int_{\widetilde{L}_{T}}^{1} \int_{\widetilde{L}_{x}}^{\widetilde{L}_{x}} \frac{\partial \mathrm{u}^{(1)}}{\partial x} \phi_{a}^{+}(y) e^{-i k_{a} x} e^{-i \bar{L}_{a} t} G_{a}(p) d x d p d y d t-$

$\lim _{\chi \rightarrow \infty} \frac{1}{2 \chi \widetilde{L}_{x}} \int_{-\widetilde{L}_{x}}^{\widetilde{L}_{x}} \int_{0}^{\chi} \int_{\widetilde{p}_{T}} \int_{-\infty}^{+\infty} \frac{\partial \mathrm{v}^{(1)}}{\partial y} \phi_{a}^{+}(y) e^{-i k_{a} x} e^{-i \varpi_{a} t} G_{a}(p) d y d p d t d x+$

$\lim _{\chi \rightarrow \infty} \frac{1}{2 \chi \widetilde{L}_{x}} \int_{-\widetilde{L}_{x}}^{\widetilde{L}_{x}} \int_{-\infty}^{+\infty} \int_{\widetilde{L}_{1}} \int_{\widetilde{p}_{T}}^{1} \frac{\partial}{\partial t} \frac{\partial}{\partial p}\left(\frac{1}{\widetilde{\bar{\sigma}}} \frac{\partial \phi^{(1)}}{\partial p}\right) \phi_{a}^{+}(y) e^{-i k_{a} x} e^{-i \omega_{a} t} G_{a}(p) d p d t d y d x=$

$\lim _{\chi \rightarrow \infty} \frac{1}{2 \chi \widetilde{L}_{x}} \int_{0}^{\chi} \int_{-\widetilde{L}_{x}}^{\widetilde{L}_{x}} \int_{\widetilde{p}_{T}}^{1}\left\langle N^{(0)} \bullet \xi_{a}^{+}(y) e^{-i k_{a} x-i \sigma_{a} t} G_{a}(p)\right\rangle d p d x d t$

Integrando por partes cada termo do lado esquerdo de (2.73), obtém-se: 
$\left.\lim _{\chi \rightarrow \infty} \frac{1}{2 \chi \widetilde{L}_{x}} \int_{-\widetilde{L}_{x}}^{\widetilde{L}_{x}} \int_{-\infty}^{+\infty} \int_{\widetilde{P}_{T}}^{1} u^{(1)} u_{a}^{+}(y) e^{-i k_{a} x} e^{-i \omega_{a} t} G_{a}(p)\right|_{t=0} ^{t=\chi} d p d y d x-$

$\lim _{\chi \rightarrow \infty} \frac{1}{2 \chi \widetilde{L}_{x}} \int_{-\widetilde{L}_{x}}^{\widetilde{L}_{x}} \int_{-\infty}^{+\infty} \int_{\widetilde{p}_{T}}^{1} \int_{0}^{\chi} u^{(1)} \frac{\partial\left(u_{a}^{+}(y) e^{-i k_{a} x} e^{-i \varpi_{a} t} G_{a}(p)\right)}{\partial t} d t d p d y d x-$

$\lim _{\chi \rightarrow \infty} \frac{1}{2 \chi \widetilde{L}_{x}} \int_{-\widetilde{L}_{x}}^{\widetilde{L}_{x}} \int_{-\infty}^{+\infty} \int_{\tilde{p}_{T}}^{1} \int_{0}^{\chi} y \mathrm{v}^{(1)} u_{a}^{+}(y) e^{-i k_{a} x} e^{-i \varpi_{a} t} G_{a}(p) d t d p d y d x+$

$\left.\lim _{\chi \rightarrow \infty} \frac{1}{2 \chi \widetilde{L}_{x}} \int_{0}^{\chi} \int_{-\infty}^{+\infty} \int_{\widetilde{p}_{T}}^{1} \phi^{(1)} u_{a}^{+}(y) e^{-i k_{a} x} e^{-i \varpi_{a} t} G_{a}(p)\right|_{-\widetilde{L}_{x}} ^{\widetilde{L}_{x}} d p d y d t-$

$\lim _{\chi \rightarrow \infty} \frac{1}{2 \chi \widetilde{L}_{x}} \int_{0}^{\chi} \int_{-\infty}^{+\infty} \int_{\widetilde{p}_{T}}^{1} \int_{\widetilde{L}_{x}}^{\widetilde{L}_{x}} \phi^{(1)} \frac{\partial\left(u_{a}^{+}(y) e^{-i k_{a} x} e^{-i \varpi_{a} t} G_{a}(p)\right)}{\partial x} d x d p d y d t+$

$\left.\lim _{\chi \rightarrow \infty} \frac{1}{2 \chi \widetilde{L}_{x}} \int_{-\widetilde{L}_{x}}^{\widetilde{L}_{x}} \int_{-\infty}^{+\infty} \int_{\widetilde{p}_{T}}^{1} \mathrm{v}^{(1)} \mathrm{v}_{a}^{+}(y) e^{-i k_{a} x} e^{-i \pi_{a} t} G_{a}(p)\right|_{t=0} ^{t=\chi} d p d y d x-$

$\lim _{\chi \rightarrow \infty} \frac{1}{2 \chi \widetilde{L}_{x}} \int_{-\widetilde{L}_{x}}^{\widetilde{L}_{x}} \int_{-\infty}^{+\infty} \int_{\tilde{p}_{T}}^{1} \int_{0}^{\chi} \mathrm{v}^{(1)} \frac{\partial\left(\mathrm{v}_{a}^{+}(y) e^{-i k_{a} x} e^{-i \sigma_{a} t} G_{a}(p)\right)}{\partial t} d t d p d y d x+$

$\lim _{\chi \rightarrow \infty} \frac{1}{2 \chi \widetilde{L}_{x}} \int_{-\widetilde{L}_{x}}^{\widetilde{L}_{x}} \int_{-\infty}^{+\infty} \int_{\tilde{p}_{T}}^{1} \int_{0}^{\chi} \mathrm{yu}^{(1)} \mathrm{v}_{a}^{+}(y) e^{-i k_{a} x} e^{-i \varpi_{a} t} G_{a}(p) d t d p d y d x+$

$\left.\lim _{\chi \rightarrow \infty} \frac{1}{2 \chi \widetilde{L}_{x}} \int_{0}^{\chi} \int_{-\widetilde{L}_{x}}^{\widetilde{L}_{x}} \int_{\widetilde{p}_{T}}^{1} \phi^{(1)} \mathrm{V}_{a}^{+}(y) e^{-i k_{a} x} e^{-i \varpi_{a} t} G_{a}(p)\right|_{-\infty} ^{+\infty} d p d x d t-$

$\lim _{\chi \rightarrow \infty} \frac{1}{2 \chi \widetilde{L}_{x}} \int_{0}^{\chi} \int_{-\infty}^{+\infty} \int_{\tilde{p}_{T}}^{1} \int_{\widetilde{L}_{x}}^{\tilde{L}_{x}} \phi^{(1)} \frac{\partial\left(\mathrm{v}_{a}^{+}(y) e^{-i k_{a} x} e^{-i \varpi_{a} t} G_{a}(p)\right)}{\partial y} d x d p d y d t-$

$\left.\lim _{\chi \rightarrow \infty} \frac{1}{2 \chi \widetilde{L}_{x}} \int_{0}^{\chi} \int_{-\infty}^{+\infty} \int_{\widetilde{p}_{T}}^{1} \mathrm{u}^{(1)} \phi_{a}^{+}(y) e^{-i k_{a} x} e^{-i \varpi_{a} t} G_{a}(p)\right|_{-\widetilde{L}_{x}} ^{\widetilde{L}_{x}} d p d y d t+$

$\lim _{\chi \rightarrow \infty} \frac{1}{2 \chi \widetilde{L}_{x}} \int_{0}^{\chi} \int_{-\infty}^{+\infty} \int_{\widetilde{p}_{T}}^{1} \int_{\widetilde{L}_{x}}^{\widetilde{L}_{x}} u^{(1)} \frac{\partial\left(\phi_{a}^{+}(y) e^{-i k_{a} x} e^{-i \omega_{a} t} G_{a}(p)\right)}{\partial x} d x d p d y d t-$

$\left.\lim _{\chi \rightarrow \infty} \frac{1}{2 \chi \widetilde{L}_{x}} \int_{0}^{\chi} \int_{-\widetilde{L}_{x}}^{\widetilde{L}_{x}} \int_{\widetilde{p}_{T}}^{1} \mathrm{v}^{(1)} \phi_{a}^{+}(y) e^{-i k_{a} x} e^{-i \sigma_{a} t} G_{a}(p)\right|_{-\infty} ^{+\infty} d p d x d t+$

$\lim _{\chi \rightarrow \infty} \frac{1}{2 \chi \widetilde{L}_{x}} \int_{0}^{\chi} \int_{-\infty}^{+\infty} \int_{\widetilde{p}_{T}} \int_{\widetilde{L}_{x}}^{\widetilde{L}_{x}} \mathrm{v}^{(1)} \frac{\partial\left(\phi_{a}^{+}(y) e^{-i k_{a} x} e^{-i \bar{L}_{a} t} G_{a}(p)\right)}{\partial y} d x d p d y d t+$

$\lim _{\chi \rightarrow \infty} \frac{1}{2 \chi \widetilde{L}_{x}} \int_{-\widetilde{L}_{x}}^{\widetilde{L}_{x}} \int_{-\infty 0}^{+\infty} \int_{\widetilde{L}_{x}}\left[\left.\frac{1}{\widetilde{\widetilde{\sigma}}} \phi_{a}^{+}(y) e^{-i k_{a} x} e^{-i \sigma_{a} t} G_{a}(p) \frac{\partial}{\partial p} \frac{\partial \phi^{(1)}}{\partial t}\right|_{\widetilde{p}_{T}} ^{1}-\left.\frac{1}{\widetilde{\sigma}} \phi_{a}^{+}(y) e^{-i k_{a} x} e^{-i \sigma_{a} t} \frac{d G_{a}}{d p} \frac{\partial \phi^{(1)}}{\partial t}\right|_{\widetilde{p}_{T}} ^{1}\right] d t d y d x-$

$\lim _{\chi \rightarrow \infty} \frac{1}{2 \chi \widetilde{L}_{x}} \int_{-\widetilde{L}_{x}}^{\widetilde{L}_{x}} \int_{-\infty}^{+\infty} \int_{0} \int_{\widetilde{p}_{T}}^{1} \phi^{(1)} \frac{\partial\left(\phi_{a}^{+}(y) e^{-i k_{a} x} e^{-i \sigma_{a} t}\right)}{\partial t} \frac{d}{d p}\left(\frac{1}{\widetilde{\sigma}} \frac{d G_{a}(p)}{d p}\right) d p d t d y d x+$

$\left.\lim _{\chi \rightarrow \infty} \frac{1}{2 \chi \widetilde{L}_{x}} \int_{-\widetilde{L}_{x}}^{\widetilde{L}_{x}} \int_{-\infty}^{+\infty} \int_{\widetilde{p}_{T}}^{1} \phi^{(1)} \phi_{a}^{+}(y) e^{-i k_{a} x} e^{-i \bar{\omega}_{a} t} \frac{d}{d p}\left(\frac{1}{\widetilde{\widetilde{\sigma}}} \frac{d G_{a}(p)}{d p}\right)\right|_{t=0} ^{t=\chi} d p d y d x=$ 


$$
\lim _{\chi \rightarrow \infty} \frac{1}{2 \chi \widetilde{L}_{x}} \int_{0}^{\chi} \int_{-\widetilde{L}_{x}}^{\widetilde{L}_{x}} \int_{\widetilde{p}_{T}}^{1}\left\langle N^{(0)} \bullet \xi_{a}^{+}(y) e^{-i k_{a} x-i \varpi_{a} t} G_{a}(p)\right\rangle d p d x d t
$$

As condições de contorno em x e em y do problema de $\mathrm{O}(\varepsilon)$ também são dadas por (2.10), assim como as do problema de $\mathrm{O}(1)$. Conseqüentemente, usando estas condições de fronteira, é possível reescrever a equação (2.74) da seguinte forma:

$$
\begin{aligned}
& \lim _{\chi \rightarrow \infty} \frac{1}{2 \chi \widetilde{L}_{x}} \int_{0}^{\chi} \int_{-\widetilde{L}_{x}}^{\widetilde{L}_{x}} \int_{\widetilde{p}_{T}}^{1}\left\langle\mathfrak{J}^{+} \xi_{a}^{+}(y) e^{-i k_{a} x-i \varpi_{a} t} G_{a}(p) \bullet \xi^{(1)}\right\rangle d p d x d t+ \\
& \left.\lim _{\chi \rightarrow \infty} \frac{1}{2 \chi \widetilde{L}_{x}} \int_{-\widetilde{L}_{x}}^{\widetilde{L}_{x}} \int_{-\infty}^{+\infty} \int_{\widetilde{p}_{T}}^{1} u^{(1)} u_{a}^{+}(y) e^{-i k_{a} x} e^{-i \varpi_{a} t} G_{a}(p)\right|_{t=0} ^{t=\chi} d p d y d x+
\end{aligned}
$$

$\left.\lim _{\chi \rightarrow \infty} \frac{1}{2 \chi \widetilde{L}_{x}} \int_{-\widetilde{L}_{x}}^{\widetilde{L}_{x}} \int_{-\infty}^{+\infty} \int_{\widetilde{p}_{T}}^{1} \mathrm{v}^{(1)} \mathrm{v}_{a}^{+}(y) e^{-i k_{a} x} e^{-i \sigma_{a} t} G_{a}(p)\right|_{t=0} ^{t=\chi} d p d y d x+$

$\left.\lim _{\chi \rightarrow \infty} \frac{1}{2 \chi \widetilde{L}_{x}} \int_{-\widetilde{L}_{x}}^{\widetilde{L}_{x}} \int_{-\infty}^{+\infty} \int_{\tilde{T}_{T}}^{1} \phi^{(1)} \phi_{a}^{+}(y) e^{-i k_{a} x} e^{-i \varpi_{a} t} \frac{d}{d p}\left(\frac{1}{\widetilde{\widetilde{\sigma}}} \frac{d G_{a}(p)}{d p}\right)\right|_{t=0} ^{t=\chi} d p d y d x+$

$\lim _{\chi \rightarrow \infty} \frac{1}{2 \chi \widetilde{L}_{x}} \int_{-\widetilde{L}_{x}}^{\widetilde{L}_{x}} \int_{-\infty}^{+\infty} \int_{0}\left[\left.\frac{1}{\widetilde{\sigma}} \phi_{a}^{+}(y) e^{-i k_{a} x} e^{-i \sigma_{a} t} G_{a}(p) \frac{\partial}{\partial p} \frac{\partial \phi^{(1)}}{\partial t}\right|_{\widetilde{p}_{T}} ^{1}-\left.\frac{1}{\widetilde{\sigma}} \phi_{a}^{+}(y) e^{-i k_{a} x} e^{-i \varpi_{a} t} \frac{d G_{a}}{d p} \frac{\partial \phi^{(1)}}{\partial t}\right|_{\widetilde{p}_{T}} ^{1}\right] d t d y d x=$

$\lim _{\chi \rightarrow \infty} \frac{1}{2 \chi \widetilde{L}_{x}} \int_{0}^{\chi} \int_{-\widetilde{L}_{x}}^{\widetilde{L}_{x}} \int_{\widetilde{p}_{T}}^{1}\left\langle N^{(0)} \bullet \xi_{a}^{+}(y) e^{-i k_{a} x-i \varpi_{a} t} G_{a}(p)\right\rangle d p d x d t$

Uma vez que $\xi_{a}^{+}(y) e^{-i k_{a} x-i \varpi_{a} t} G_{a}(p)$ é solução do problema de $\mathrm{O}(1)$ adjunto e, conseqüentemente, satisfaz às equações (2.58), o primeiro termo no lado esquerdo de (2.75) é nulo. Assim, usando também as condições de fronteira do problema de $\mathrm{O}(1)$ na vertical dadas por (2.33), a equação (2.75) torna-se: 
$\left.\lim _{\chi \rightarrow \infty} \frac{1}{2 \chi \widetilde{L}_{x}} \int_{-\widetilde{L}_{x}}^{\widetilde{L}_{x}} \int_{-\infty}^{+\infty} \int_{\tilde{L}_{T}}^{1} u^{(1)} u_{a}^{+}(y) e^{-i k_{a} x} e^{-i \varpi_{a} t} G_{a}(p)\right|_{t=0} ^{t=\chi} d p d y d x+$

$\left.\lim _{\chi \rightarrow \infty} \frac{1}{2 \chi \widetilde{L}_{x}} \int_{-\widetilde{L}_{x}}^{\widetilde{L}_{x}} \int_{-\infty}^{+\infty} \int_{\widetilde{L}_{T}}^{1} \mathrm{v}^{(1)} \mathbf{v}_{a}^{+}(y) e^{-i k_{a} x} e^{-i \omega_{a} t} G_{a}(p)\right|_{t=0} ^{t=\chi} d p d y d x+$

$\left.\lim _{\chi \rightarrow \infty} \frac{1}{2 \chi \widetilde{L}_{x}} \int_{-\widetilde{L}_{x}}^{\widetilde{L}_{x}} \int_{-\infty}^{+\infty} \int_{\widetilde{p}_{T}}^{1} \phi^{(1)} \phi_{a}^{+}(y) e^{-i k_{a} x} e^{-i \sigma_{a} t} \frac{d}{d p}\left(\frac{1}{\widetilde{\widetilde{\sigma}}} \frac{d G_{a}(p)}{d p}\right)\right|_{t=0} ^{t=\chi} d p d y d x+$

$\left.\lim _{\chi \rightarrow \infty} \frac{1}{2 \chi \widetilde{L}_{x}} \int_{-\widetilde{L}_{x}}^{\widetilde{L}_{x}} \int_{-\infty 0}^{+\infty \chi} \int_{0}\left[\frac{1}{\widetilde{\sigma}} \phi_{a}^{+}(y) e^{-i k_{a} x} e^{-i \Phi_{a} t} G_{a}(p)\left(\frac{\partial}{\partial p} \frac{\partial \phi^{(1)}}{\partial t}+\widetilde{\widetilde{\sigma} \widetilde{\rho}} \frac{\partial \phi^{(1)}}{\partial t}\right)\right]\right|_{\widetilde{p}_{T}} ^{1} d t d y d x=$

$\lim _{\chi \rightarrow \infty} \frac{1}{2 \chi \widetilde{L}_{x}} \int_{0}^{\chi} \int_{-\widetilde{L}_{x}}^{\widetilde{L}_{x}} \int_{\widetilde{p}_{T}}^{1}\left\langle N^{(0)} \bullet \xi_{a}^{+}(y) e^{-i k_{a} x-i \varpi_{a} t} G_{a}(p)\right) d p d x d t$

Por outro lado, a equação da termodinâmica (2.18d) na $O(\varepsilon)$ é escrita da seguinte forma:

$\frac{\partial}{\partial t} \frac{\partial \phi^{(1)}}{\partial p}+\frac{\partial}{\partial \tau} \frac{\partial \phi^{(0)}}{\partial p}+u^{(0)} \frac{\partial}{\partial x} \frac{\partial \phi^{(0)}}{\partial p}+\mathrm{v}^{(0)} \frac{\partial}{\partial y} \frac{\partial \phi^{(0)}}{\partial p}+F \omega^{(0)} \frac{\partial^{2} \phi^{(0)}}{\partial p^{2}}+\frac{F \omega^{(0)}}{p}(1-\kappa) \frac{\partial \phi^{(0)}}{\partial p}+F \tilde{\widetilde{\sigma}} \omega^{(1)}=0$

Aplicando a equação (2.77) acima em $\mathrm{p}=1 \mathrm{e}$ em $\mathrm{p}=\widetilde{p}_{T}$ e usando as equações (2.70) e as condições de fronteira verticais (2.33) associadas à solução do problema de $\mathrm{O}(1)$, chegase às seguintes expressões:

$$
\begin{aligned}
& \left.\left(\frac{\partial}{\partial p} \frac{\partial \phi^{(1)}}{\partial t}+\widetilde{\widetilde{\sigma}} \widetilde{\rho} \frac{\partial \phi^{(1)}}{\partial t}\right)\right|_{p=1}=-\left.F \omega^{(0)} \frac{\partial^{2} \phi^{(0)}}{\partial p^{2}}\right|_{p=1}+\left.\widetilde{\bar{\rho}}_{0} \widetilde{\bar{\sigma}}(1-\kappa) F \omega^{(0)} \phi^{(0)}\right|_{p=1}+\left.\widetilde{\bar{\sigma}}^{2} \widetilde{\bar{\rho}}_{0}^{2} F \omega^{(0)} \phi^{(0)}\right|_{p=1}- \\
& \left.\widetilde{\widetilde{\sigma}}_{0}^{2} \phi^{(0)}\right|_{p=1} \frac{\partial}{\partial p}\left[\frac{\partial \phi^{(0)}}{\partial t}-\left.\frac{F \omega^{(0)}}{\widetilde{\bar{\rho}}}\right|_{p=1}=-\left.F \omega^{(0)} \frac{\partial^{2} \phi^{(0)}}{\partial p^{2}}\right|_{p=1}+\left.\widetilde{\bar{\rho}}_{0} \widetilde{\bar{\sigma}}(1-\kappa) F \omega^{(0)} \phi^{(0)}\right|_{p=1}+\right. \\
& \left.\widetilde{\bar{\sigma}}^{2} \widetilde{\bar{\rho}}_{0}^{2} F \omega^{(0)} \phi^{(0)}\right|_{p=1}+\left.\left.\widetilde{\bar{\sigma}}^{2} \widetilde{\bar{\rho}}_{0}^{3} \phi^{(0)}\right|_{p=1} \frac{\partial \phi^{(0)}}{\partial t}\right|_{p=1}+\left.\left.\widetilde{\widetilde{\sigma} \widetilde{\rho}_{0}} \phi^{(0)}\right|_{p=1} F \frac{\partial \omega^{(0)}}{\partial p}\right|_{p=1}-\left.\left.\left.\widetilde{\bar{\sigma}} \frac{d \tilde{\bar{\rho}}}{d p}\right|_{p=1} \phi^{(0)}\right|_{p=1} F \omega^{(0)}\right|_{p=1}
\end{aligned}
$$




$$
\begin{aligned}
& \left.\left(\frac{\partial}{\partial p} \frac{\partial \phi^{(1)}}{\partial t}+\widetilde{\bar{\sigma}} \widetilde{\rho} \frac{\partial \phi^{(1)}}{\partial t}\right)\right|_{p=\widetilde{p}_{T}}=-\left.F \omega^{(0)} \frac{\partial^{2} \phi^{(0)}}{\partial p^{2}}\right|_{p=\widetilde{p}_{T}}+\left.\widetilde{\bar{\rho}}_{T} \widetilde{\bar{\sigma}}(1-\kappa) \frac{F \omega^{(0)}}{\widetilde{p}_{T}} \phi^{(0)}\right|_{p=\widetilde{p}_{T}}+\left.\widetilde{\bar{\sigma}}^{2} \widetilde{\bar{\rho}}_{T}^{2} F \omega^{(0)} \phi^{(0)}\right|_{p=\widetilde{p}_{T}}+ \\
& \left.\widetilde{\sigma}_{T}^{2} \phi^{(0)}\right|_{p=\widetilde{p}_{T}} \frac{\partial}{\partial p}\left[\frac{\partial \phi^{(0)}}{\partial t}-\left.\frac{F \omega^{(0)}}{\widetilde{\bar{\rho}}}\right|_{p=\widetilde{p}_{T}}=-\left.F \omega^{(0)} \frac{\partial^{2} \phi^{(0)}}{\partial p^{2}}\right|_{p=\widetilde{p}_{T}}+\left.\widetilde{\bar{\rho}}_{T} \widetilde{\bar{\sigma}}(1-\kappa) \frac{F \omega^{(0)}}{\widetilde{p}_{T}} \phi^{(0)}\right|_{p=\widetilde{p}_{T}}+\right. \\
& \left.\widetilde{\bar{\sigma}}^{2} \widetilde{\bar{\rho}}_{T}^{2} F \omega^{(0)} \phi^{(0)}\right|_{p=\widetilde{p}_{T}}-\left.\left.\widetilde{\bar{\sigma}}^{2} \widetilde{\bar{\rho}}_{T}^{3} \phi^{(0)}\right|_{p=\widetilde{p}_{T}} \frac{\partial \phi^{(0)}}{\partial t}\right|_{p=\widetilde{p}_{T}}-\left.\left.\widetilde{\sigma}_{\bar{\rho}_{T}} \phi^{(0)}\right|_{p=\widetilde{p}_{T}} F \frac{\partial \omega^{(0)}}{\partial p}\right|_{p=\widetilde{p}_{T}}+\left.\left.\left.\widetilde{\bar{\sigma}} \frac{d \bar{\rho}}{d p}\right|_{p=\widetilde{p}_{T}} \phi^{(0)}\right|_{p=\widetilde{p}_{T}} F \omega^{(0)}\right|_{p=\widetilde{p}_{T}}
\end{aligned}
$$

A variável $F \omega^{(0)}$ em $\mathrm{p}=1$ e em $\mathrm{p}=\widetilde{p}_{T}$ nas equações acima pode ser escrita em função do geopotencial usando as relações (2.26). Além disso, usando a equação da continuidade para a $\mathrm{O}(1)$ dada por

$$
F \frac{\partial \omega^{(0)}}{\partial p}=-\left(\frac{\partial u^{(0)}}{\partial x}+\frac{\partial \mathrm{v}^{(0)}}{\partial y}\right)
$$

é possível reescrever as equações (2.78) da seguinte forma:

$$
\begin{aligned}
& \left(\frac{\partial}{\partial p} \frac{\partial \phi^{(1)}}{\partial t}+\left.\widetilde{\widetilde{\sigma \rho}} \frac{\partial \phi^{(1)}}{\partial t}\right|_{p=1}=-\left.\left.\widetilde{\bar{\rho}}_{0} \frac{\partial \phi^{(0)}}{\partial t}\right|_{p=1} \frac{\partial^{2} \phi^{(0)}}{\partial p^{2}}\right|_{p=1}+\left.\left.\phi^{(0)}\right|_{p=1} \frac{\partial \phi^{(0)}}{\partial t}\right|_{p=1}\left[(1-\kappa){\widetilde{\sigma \bar{\rho}_{0}}}^{2}+2 \widetilde{\bar{\sigma}}^{2} \widetilde{\bar{\rho}}_{0}^{3}-\left.\widetilde{\widetilde{\sigma}} \frac{d \tilde{\bar{\rho}}}{d p}\right|_{p=1}\right]\right. \\
& -\left.\left.\widetilde{\sigma \bar{\rho}_{0}} \phi^{(0)}\right|_{p=1}\left(\frac{\partial u^{(0)}}{\partial x}+\frac{\partial v^{(0)}}{\partial y}\right)\right|_{p=1}
\end{aligned}
$$

$$
\begin{aligned}
& \left.\left(\frac{\partial}{\partial p} \frac{\partial \phi^{(1)}}{\partial t}+\widetilde{\widetilde{\sigma \rho}} \frac{\partial \phi^{(1)}}{\partial t}\right)\right|_{p=\widetilde{p}_{T}}=-\left.\left.\widetilde{\bar{\rho}}_{T} \frac{\partial \phi^{(0)}}{\partial t}\right|_{p=\widetilde{p}_{T}} \frac{\partial^{2} \phi^{(0)}}{\partial p^{2}}\right|_{p=\widetilde{p}_{T}}+\left.\left.\phi^{(0)}\right|_{p=\widetilde{p}_{T}} \frac{\partial \phi^{(0)}}{\partial t}\right|_{p=\widetilde{p}_{T}}\left[\frac{(1-\kappa)}{\widetilde{p}_{T}} \widetilde{\bar{\sigma}}_{T}{ }^{2}+\left.\widetilde{\sigma}_{T} \frac{d}{d p}\right|_{p=\widetilde{p}_{T}}\right]+ \\
& \left.\left.\widetilde{\widetilde{\sigma} \bar{\rho}_{T}} \phi^{(0)}\right|_{p=\widetilde{p}_{T}}\left(\frac{\partial u^{(0)}}{\partial x}+\frac{\partial \mathrm{v}^{(0)}}{\partial y}\right)\right|_{p=\widetilde{p}_{T}}
\end{aligned}
$$

Da mesma maneira, integrando a equação da continuidade para a $\mathrm{O}(1)$ de $\mathrm{p}=\widetilde{p}_{T}$ até uma pressão p arbitrária, e usando a condição de fronteira (2.26b), obtém-se 


$$
F \omega^{(0)}(x, y, p, t, \tau)=\left.\widetilde{\bar{\rho}}_{T} \frac{\partial \phi^{(0)}}{\partial t}\right|_{p=\widetilde{p}_{T}}-\int_{\widetilde{p}_{T}}^{p}\left(\frac{\partial u^{(0)}}{\partial x}+\frac{\partial \mathrm{v}^{(0)}}{\partial y}\right) d p
$$

Assim, usando as equações (2.80a,b) e (2.81) em (2.76), tem-se que

$$
\begin{aligned}
& \left.\lim _{\chi \rightarrow \infty} \frac{1}{2 \chi \widetilde{L}_{x}} \int_{-\widetilde{L}_{x}}^{\widetilde{L}_{x}} \int_{-\infty}^{+\infty} \int_{\widetilde{p}_{T}}^{1} u^{(1)} u_{a}^{+}(y) e^{-i k_{a} x} e^{-i \sigma_{a} t} G_{a}(p)\right|_{t=0} ^{t=\chi} d p d y d x+ \\
& \left.\lim _{\chi \rightarrow \infty} \frac{1}{2 \chi \widetilde{L}_{x}} \int_{-\widetilde{L}_{x}}^{\widetilde{L}_{x}} \int_{-\infty}^{+\infty} \int_{\widetilde{p}_{T}}^{1} \mathrm{v}^{(1)} \mathrm{v}_{a}^{+}(y) e^{-i k_{a} x} e^{-i \sigma_{a} t} G_{a}(p)\right|_{t=0} ^{t=\chi} d p d y d x+ \\
& \left.\lim _{\chi \rightarrow \infty} \frac{1}{2 \chi \widetilde{L}_{x}} \int_{-\widetilde{L}_{x}}^{\widetilde{L}_{x}} \int_{-\infty}^{+\infty} \int_{\widetilde{p}_{T}}^{1} \phi^{(1)} \phi_{a}^{+}(y) e^{-i k_{a} x} e^{-i \sigma_{a} t} \frac{d}{d p}\left(\frac{1}{\widetilde{\bar{\sigma}}} \frac{d G_{a}(p)}{d p}\right)\right|_{t=0} ^{t=\chi} d p d y d x+
\end{aligned}
$$

$\left.\lim _{\chi \rightarrow \infty} \frac{1}{2 \chi \widetilde{L}_{x}} \int_{-\widetilde{L}_{x}}^{\widetilde{L}_{x}} \int_{-\infty}^{+\infty} \int\left\{\phi_{a}^{+}(y) e^{-i k_{a} x} e^{-i \sigma_{a} t} G_{a}(p)\left[-\widetilde{\bar{\rho}} \frac{\partial \phi^{(0)}}{\partial t} \frac{1}{\widetilde{\widetilde{\sigma}}} \frac{\partial^{2} \phi^{(0)}}{\partial p^{2}}+\widetilde{\bar{\Gamma}} \phi^{(0)} \frac{\partial \phi^{(0)}}{\partial t}-\Lambda \tilde{\bar{\rho}}^{(0)}\left(\frac{\partial u^{(0)}}{\partial x}+\frac{\partial \mathrm{v}^{(0)}}{\partial y}\right)\right]\right\}\right|_{\widetilde{p}_{T}} ^{1} d t d y d x=$ $\lim _{\chi \rightarrow \infty} \frac{1}{2 \chi \widetilde{L}_{x}} \int_{0}^{\chi} \int_{-\widetilde{L}_{x}}^{\widetilde{L}_{x}} \int_{\widetilde{p}_{T}}^{1}\left\langle N \bullet \xi_{a}^{+}(y) e^{-i k_{a} x-i \varpi_{a} t} G_{a}(p)\right\rangle d p d x d t$

onde o parâmetro $\widetilde{\bar{\Gamma}}$ em (2.82) é definido como

$$
\widetilde{\bar{\Gamma}}=\frac{(1-\kappa)}{p} \widetilde{\bar{\rho}}^{2}+\widetilde{\bar{\sigma}}^{3}(1+\Lambda)-\Lambda \widetilde{\bar{\rho}} \frac{d \widetilde{\bar{\rho}}}{d p}
$$

sendo que o parâmetro $\Lambda$ em (2.83) e (2.82) acima é dado por

$$
\Lambda=\left\{\begin{array}{rll}
1, & \text { se } & p=1 \\
-1, & \text { se } & p=\widetilde{p}_{T}
\end{array}\right.
$$

$\mathrm{O}$ vetor $\mathrm{N}$ no lado direito de (2.82) neste caso é dado por

$$
\mathrm{N}=\left[\mathrm{N}_{1}, \mathrm{~N}_{2}, \mathrm{~N}_{3}\right]^{\mathrm{T}}
$$

sendo que 


$$
\begin{aligned}
& N_{1}=-\frac{\partial u^{(0)}}{\partial \tau}-u^{(0)} \frac{\partial u^{(0)}}{\partial x}-\mathbf{v}^{(0)} \frac{\partial u^{(0)}}{\partial y}-\left.{\widetilde{\rho_{T}}}_{T} \frac{\partial \phi^{(0)}}{\partial t}\right|_{p=\widetilde{p}_{T}} \frac{\partial u^{(0)}}{\partial p}+\int_{\widetilde{p}_{T}}^{p}\left(\frac{\partial u^{(0)}}{\partial x}+\frac{\partial \mathbf{v}^{(0)}}{\partial y}\right) d p \frac{\partial u^{(0)}}{\partial p} \\
& N_{2}=-\frac{\partial \mathbf{v}^{(0)}}{\partial \tau}-u^{(0)} \frac{\partial \mathbf{v}^{(0)}}{\partial x}-\mathbf{v}^{(0)} \frac{\partial \mathbf{v}^{(0)}}{\partial y}-\left.\widetilde{\widetilde{\rho}}_{T} \frac{\partial \phi^{(0)}}{\partial t}\right|_{p=\widetilde{p}_{T}} \frac{\partial \mathbf{v}^{(0)}}{\partial p}+\int_{\widetilde{p}_{T}}^{p}\left(\frac{\partial u^{(0)}}{\partial x}+\frac{\partial \mathbf{v}^{(0)}}{\partial y}\right) d p \frac{\partial \mathbf{v}^{(0)}}{\partial p}
\end{aligned}
$$

$$
\begin{aligned}
& N_{3}=-\frac{\partial}{\partial \tau} \frac{\partial}{\partial p}\left(\frac{1}{\widetilde{\bar{\sigma}}} \frac{\partial \phi^{(0)}}{\partial p}\right)-\frac{\partial}{\partial p}\left[\frac{u^{(0)}}{\widetilde{\sigma}} \frac{\partial}{\partial x} \frac{\partial \phi^{(0)}}{\partial p}+\frac{\mathrm{v}^{(0)}}{\widetilde{\bar{\sigma}}} \frac{\partial}{\partial y} \frac{\partial \phi^{(0)}}{\partial p}\right]+\frac{1}{\widetilde{\bar{\sigma}}} \frac{\partial^{2} \phi^{(0)}}{\partial p^{2}}\left(\frac{\partial u^{(0)}}{\partial x}+\frac{\partial \mathrm{v}^{(0)}}{\partial y}\right)- \\
& \left.\widetilde{\bar{\rho}}_{T} \frac{\partial \phi^{(0)}}{\partial t}\right|_{p=\widetilde{p}_{T}} \frac{1}{\widetilde{\sigma}} \frac{\partial}{\partial p} \frac{\partial^{2} \phi^{(0)}}{\partial p^{2}}+\frac{1}{\widetilde{\sigma}} \frac{\partial}{\partial p} \frac{\partial^{2} \phi^{(0)}}{\partial p^{2}} \int_{\widetilde{p}_{T}}^{p}\left(\frac{\partial u^{(0)}}{\partial x}+\frac{\partial \mathrm{v}^{(0)}}{\partial y}\right) d p+\frac{(1-\kappa)}{p \widetilde{\sigma}} \frac{\partial \phi^{(0)}}{\partial p}\left(\frac{\partial u^{(0)}}{\partial x}+\frac{\partial \mathrm{v}^{(0)}}{\partial y}\right)+ \\
& \left.\widetilde{\widetilde{\rho}_{T}} \frac{\partial \phi^{(0)}}{\partial t}\right|_{p=\widetilde{p}_{T}} \frac{(1-\kappa)}{p^{2} \widetilde{\sigma}} \frac{\partial \phi^{(0)}}{\partial p}-\frac{(1-\kappa)}{p^{2} \widetilde{\sigma}} \frac{\partial \phi^{(0)}}{\partial p} \int_{\widetilde{p}_{T}}^{p}\left(\frac{\partial u^{(0)}}{\partial x}+\frac{\partial \mathrm{v}^{(0)}}{\partial y}\right) d p-\left.\widetilde{\bar{\rho}}_{T} \frac{\partial \phi^{(0)}}{\partial t}\right|_{p=\widetilde{p}_{T}} \frac{(1-\kappa)}{p \widetilde{\sigma}} \frac{\partial^{2} \phi^{(0)}}{\partial p^{2}}+ \\
& \frac{(1-\kappa)}{p \tilde{\sigma}} \frac{\partial^{2} \phi^{(0)}}{\partial p^{2}} \int_{\widetilde{p}_{T}}^{p}\left(\frac{\partial u^{(0)}}{\partial x}+\frac{\partial \mathrm{v}^{(0)}}{\partial y}\right) d p
\end{aligned}
$$

Substituindo $\mathrm{u}^{(0)}, \mathrm{v}^{(0)}$ e $\phi^{(0)}$ em (2.82) e em (2.86) pela solução explícita do problema de $\mathrm{O}(1)$ dada por (2.53), é possível reescrever a condição de solvabilidade (2.72) da seguinte forma:

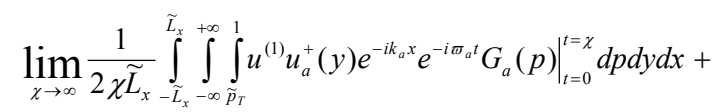

$\left.\lim _{x \rightarrow \infty} \frac{1}{2 \chi \widetilde{L}_{x}} \int_{-L_{x}-\infty}^{\tilde{L}_{x}} \int_{\tilde{p}_{T}}^{+\infty} v^{(1)} V_{a}^{+}(y) e^{-i k_{a} x} e^{-i \sigma_{a} a^{t}} G_{a}(p)\right|_{t=0} ^{t=\chi} d p d y d x+$

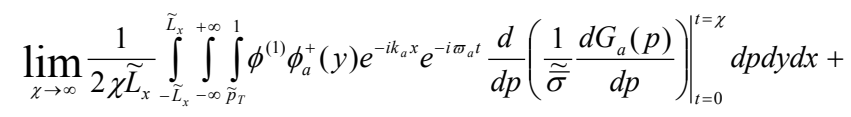

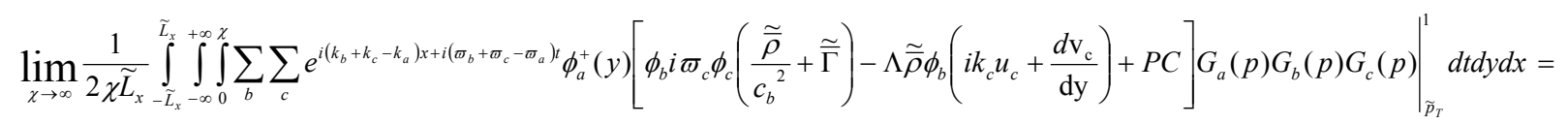
$\lim _{x \rightarrow \infty} \frac{1}{2 \chi \widetilde{L}_{x}} \int_{0}^{x} \int_{-L_{x}}^{\tilde{L}_{x}} \int_{P_{T}}^{1}\left\langle N \cdot \xi_{a}^{+}(y) e^{-i_{k} x-i \sigma_{a} t_{a}} G_{a}(p)\right) d p d x d t$ 
onde o termo PC em (2.87) representa a permutação cíclica entre os sub-índices b e c. Na equação (2.87), o vetor $\mathrm{N}$ pode ser escrito como:

$$
\mathrm{N}=\left[\mathrm{N}_{1}, \mathrm{~N}_{2}, \mathrm{~N}_{3}\right]^{\mathrm{T}}
$$

sendo que as componentes escalares $\mathrm{N}_{1}, \mathrm{~N}_{2}$ e $\mathrm{N}_{3}$ são dadas por

$$
\begin{aligned}
& N_{1}=-\sum_{a} \frac{d A_{a}}{d \tau} u_{a}(y) e^{i k_{a} x} e^{i \varpi_{a} t} G_{a}(p)- \\
& \sum_{b} \sum_{c} e^{i\left(k_{b}+k_{c}\right) x} e^{i\left(\sigma_{b}+\varpi_{c}\right) t}\left[\left(u_{b} i k_{c} u_{c}+\mathrm{v}_{\mathrm{b}} \frac{d u_{c}}{d y}\right) G_{b} G_{c}+\widetilde{\bar{\rho}}_{T} i \varpi_{b} \phi_{b} u_{c} G_{b}\left(\widetilde{p}_{T}\right) \frac{d G_{c}}{d p}-\left(i k_{b} u_{b}+\frac{d \mathrm{v}_{\mathrm{b}}}{\mathrm{dy}}\right) u_{c} \frac{d G_{c}}{d p} \int_{\widetilde{p}_{T}}^{p} G_{b} d p+P C\right]
\end{aligned}
$$

$$
\begin{aligned}
& N_{2}=-\sum_{a} \frac{d A_{a}}{d \tau} \mathrm{v}_{a}(y) e^{i k_{a} x} e^{i \sigma_{a} t} G_{a}(p)- \\
& \sum_{b} \sum_{c} e^{i\left(k_{b}+k_{c}\right) x} e^{i\left(\sigma_{b}+\varpi_{c}\right) t}\left[\left(u_{b} i k_{c} \mathrm{v}_{c}+\mathrm{v}_{\mathrm{b}} \frac{d \mathrm{v}_{c}}{d y}\right) G_{b} G_{c}+\widetilde{\bar{\rho}}_{T} i \varpi_{b} \phi_{b} \mathrm{v}_{c} G_{b}\left(\widetilde{p}_{T}\right) \frac{d G_{c}}{d p}-\left(i k_{b} u_{b}+\frac{d \mathrm{v}_{\mathrm{b}}}{\mathrm{dy}}\right) \mathrm{v}_{c} \frac{d G_{c}}{d p} \int_{\widetilde{p}_{T}}^{p} G_{b} d p+P C\right]
\end{aligned}
$$

$$
\begin{aligned}
& N_{3}=-\sum_{a} \frac{d A_{a}}{d \tau} \phi_{a}(y) e^{i k_{a} x} e^{i \sigma_{a} t}\left(-\frac{G_{a}(p)}{c_{a}{ }^{2}}\right)-
\end{aligned}
$$

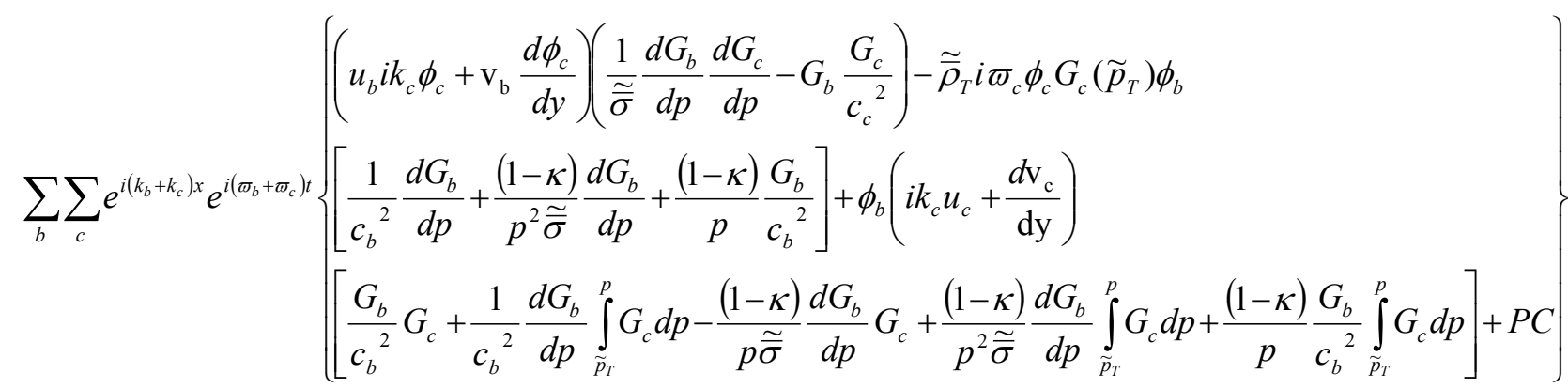

Nas integrais no tempo em (2.87) a amplitude das ondas associadas à solução do problema de $\mathrm{O}(1)$ é mantida constante ao longo da integração em $\mathrm{t}$, uma vez que, por definição, a variação temporal da solução em $\tau$ é $1 / \varepsilon$ vezes menor que a variação temporal em t. Dessa forma, a dependência da amplitude das ondas em $\tau$ é dada como incógnita e é 
determinada a partir das equações resultantes da condição de solvabilidade. $\mathrm{O}$ efeito dessa estratégia é forçar as amplitudes $A_{a}(\varepsilon t)$ das ondas a variarem de forma a tornar a condição (2.72) válida. Um procedimento alternativo foi aplicado por Bretherton (1964) que difere sutilmente em conceito mas que leva ao mesmo resultado. Basicamente, no procedimento adotado por Bretherton a operação de integração é substituída por uma média sobre um intervalo de tempo longo comparado com o período das ondas e pequeno comparado com a escala longa de tempo. Em ambos os casos, os produtos indicados em (2.87) e em (2.88) contêm somas e diferenças das freqüências como argumentos da exponencial complexa que define a estrutura em t. Se o argumento for não nulo, as integrais em (2.87) (ou a média, no caso do procedimento adotado por Bretherton) tendem à zero, devido às rápidas flutuações em t. Por outro lado, se o argumento for nulo (ou aproximadamente nulo) (ou seja, se $\varpi_{\mathrm{a}} \approx \varpi_{\mathrm{b}}+$ $\left.\varpi_{c}\right)$, as integrais no tempo em (2.87) tendem a 1. Além disso, se esta condição de ressonância para as freqüências temporais for satisfeita, os três primeiros termos no lado esquerdo de (2.87) se anulam, uma vez que, de acordo com as equações (2.67)-(2.70), a solução do problema de $\mathrm{O}(\varepsilon)$ representada pelo vetor $\xi^{(1)}=\left[\mathrm{u}^{(1)}, \mathrm{v}^{(1)}, \phi^{(1)}\right]^{\mathrm{T}}$ neste caso ressonante possui a mesma freqüência temporal de oscilação em t do termo não homogêneo representado por N. Logo, considerando somente os tripletos de ondas que satisfazem à condição de ressonância $\varpi_{\mathrm{a}}=\varpi_{\mathrm{b}}+\varpi_{\mathrm{c}}$, tem-se que a condição de solvabilidade (2.72) é dada por:

$$
\begin{aligned}
& \left.\int_{-\widetilde{L}_{x}}^{\widetilde{L}_{x}} \int_{-\infty}^{+\infty} \sum_{b} \sum_{c} e^{i\left(k_{b}+k_{c}-k_{a}\right) x} \phi_{a}^{+}(y)\left[\phi_{b} i \varpi_{c} \phi_{c}\left(\frac{\widetilde{\bar{\rho}}}{c_{b}{ }^{2}}+\widetilde{\bar{\Gamma}}\right)-\Lambda \widetilde{\bar{\rho}} \phi_{b}\left(i k_{c} u_{c}+\frac{d \mathrm{v}_{\mathrm{c}}}{\mathrm{dy}}\right)+P C\right] G_{a}(p) G_{b}(p) G_{c}(p)\right|_{\widetilde{p}_{T}} ^{1} d y d x= \\
& \int_{-\widetilde{L}_{x}}^{\widetilde{L}_{x}} \int_{\widetilde{p}_{T}}^{1}\left\langle N \bullet \xi_{a}^{+}(y) e^{-i k_{a} x} G_{a}(p)\right) d p d x
\end{aligned}
$$

sendo o vetor $\mathrm{N}$ neste caso dado por (2.88a) com as componentes escalares escritas como

$$
\begin{aligned}
& N_{1}=-\sum_{a} \frac{d A_{a}}{d \tau} u_{a}(y) e^{i k_{a} x} G_{a}(p)- \\
& \sum_{b} \sum_{c} e^{i\left(k_{b}+k_{c}\right) x}\left[\left(u_{b} i k_{c} u_{c}+\mathrm{v}_{\mathrm{b}} \frac{d u_{c}}{d y}\right) G_{b} G_{c}+\widetilde{\bar{\rho}}_{T} i \varpi_{b} \phi_{b} u_{c} G_{b}\left(\widetilde{p}_{T}\right) \frac{d G_{c}}{d p}-\left(i k_{b} u_{b}+\frac{d \mathrm{v}_{\mathrm{b}}}{\mathrm{dy}}\right) u_{c} \frac{d G_{c}}{d p} \int_{\widetilde{p}_{T}}^{p} G_{b} d p+P C\right]
\end{aligned}
$$




$$
\begin{aligned}
& N_{2}=-\sum_{a} \frac{d A_{a}}{d \tau} \mathrm{v}_{a}(y) e^{i k_{a} x} G_{a}(p)- \\
& \sum_{b} \sum_{c} e^{i\left(k_{b}+k_{c}\right) x}\left[\left(u_{b} i k_{c} \mathrm{v}_{c}+\mathrm{v}_{\mathrm{b}} \frac{d \mathrm{v}_{c}}{d y}\right) G_{b} G_{c}+\widetilde{\bar{\rho}}_{T} i \varpi_{b} \phi_{b} \mathrm{v}_{c} G_{b}\left(\widetilde{p}_{T}\right) \frac{d G_{c}}{d p}-\left(i k_{b} u_{b}+\frac{d \mathrm{v}_{\mathrm{b}}}{\mathrm{dy}}\right) \mathrm{v}_{c} \frac{d G_{c}}{d p} \int_{\widetilde{p}_{T}}^{p} G_{b} d p+P C\right]
\end{aligned}
$$

$$
\begin{aligned}
& N_{3}=-\sum_{a} \frac{d A_{a}}{d \tau} \phi_{a}(y) e^{i k_{a} x}\left(-\frac{G_{a}(p)}{c_{a}{ }^{2}}\right)- \\
& \sum_{b} \sum_{c} e^{i\left(k_{b}+k_{c}\right) x}\left\{\begin{array}{l}
\left(u_{b} i k_{c} \phi_{c}+\mathrm{v}_{\mathrm{b}} \frac{d \phi_{c}}{d y}\right)\left(\frac{1}{\widetilde{\widetilde{\sigma}}} \frac{d G_{b}}{d p} \frac{d G_{c}}{d p}-G_{b} \frac{G_{c}}{c_{c}^{2}}\right)-\widetilde{\bar{\rho}}_{T} i \varpi_{c} \phi_{c} G_{c}\left(\widetilde{p}_{T}\right) \phi_{b} \\
\left.\frac{c_{b}}{d p}+\frac{(1-\kappa)}{p^{2} \widetilde{\widetilde{\sigma}}} \frac{d G_{b}}{d p}+\frac{(1-\kappa)}{p} \frac{G_{b}}{c_{b}{ }^{2}}\right]+\phi_{b}\left(i k_{c} u_{c}+\frac{d \mathrm{v}_{\mathrm{c}}}{\mathrm{dy}}\right) \\
{\left[\frac{G_{b}}{c_{b}^{2}} G_{c}+\frac{1}{c_{b}{ }^{2}} \frac{d G_{b}}{d p} \int_{\widetilde{p}_{T}}^{p} G_{c} d p-\frac{(1-\kappa)}{p \widetilde{\widetilde{\sigma}}} \frac{d G_{b}}{d p} G_{c}+\frac{(1-\kappa)}{p^{2} \widetilde{\widetilde{\sigma}}} \frac{d G_{b}}{d p} \int_{\widetilde{p}_{T}}^{p} G_{c} d p+\frac{(1-\kappa)}{p} \frac{G_{b}}{c_{b}^{2}} \int_{\widetilde{p}_{T}}^{p} G_{c} d p\right]+P C}
\end{array}\right\}
\end{aligned}
$$

Finalmente, usando a definição das autofunções $\xi_{\mathrm{a}}^{+}(\mathrm{y})$ do problema adjunto dada por (2.65), a ortogonalidade das funções trigonométricas complexas no intervalo $\left[-\widetilde{L}_{x}, \widetilde{L}_{x}\right]$, a ortogonalidade das autofunções $\xi_{\mathrm{a}}(\mathrm{y})$ em $(-\infty,+\infty)$ e das autofunções $\mathrm{G}_{\mathrm{a}}(\mathrm{p})$ no intervalo $\left[\tilde{p}_{T}, 1\right]$, obtém-se o seguinte sistema de equações diferenciais ordinárias que governa a evolução na escala longa de tempo da amplitude de um determinado modo a devida à todas as interações triádicas ressonantes possíveis envolvendo esse modo a:

$$
c_{a}^{2} \frac{d A_{a}}{d \tau}=\sum_{b} \sum_{c} A_{b} A_{c} \eta_{a}^{b c}
$$

onde $\mathbf{a}=(\mathrm{m}, \mathrm{k}, \mathrm{n}, \mathrm{r})$ indica um modo em particular, sendo que $\mathrm{m}$ caracteriza a estrutura vertical, $\mathrm{k}$ é o número de onda em $\mathrm{x}$, $\mathrm{n}$ o modo meridional, que caracteriza a estrutura das autofunções em y, e $\mathrm{r}$ distingue os tipos de onda permitidos, como discutido anteriormente. Em (2.91) acima, $\eta_{\mathrm{a}}{ }^{\mathrm{bc}}$ representa o coeficiente de interação entre três modos ressonantes $\mathbf{a}, \mathbf{b}$ e c quaisquer e é dado pela seguinte expressão 


$$
\begin{aligned}
& \eta_{a}^{b c}=-\delta_{a b c}\left\langle\vec{B} \bullet \xi_{a}(y)\right\rangle- \\
& \left.\delta_{a b c} \int_{-\infty}^{+\infty}\left\{\phi_{a}(y)\left[\phi_{b} i \varpi_{c} \phi_{c}\left(\frac{\widetilde{\bar{\rho}}}{c_{b}{ }^{2}}+\widetilde{\bar{\Gamma}}\right)-\Lambda \widetilde{\bar{\rho}} \phi_{b}\left(i k_{c} u_{c}+\frac{d \mathrm{v}_{\mathrm{c}}}{\mathrm{dy}}\right)+P C\right] \frac{G_{a}(p) G_{b}(p) G_{c}(p)}{\left\|G_{a}\right\|^{2}}\right]\right|_{1} ^{\widetilde{P}_{T}} d y
\end{aligned}
$$

onde $<\bullet>$ acima indica o produto interno definido pela expressão (2.47) e o vetor $\vec{B}$ é dado pela seguinte expressão:

$$
\vec{B}=\left[\begin{array}{l}
\left(u_{b} i k_{c} u_{c}+\mathrm{v}_{\mathrm{b}} \frac{d u_{c}}{d y}\right) \alpha_{a}^{b c}+i \varpi_{b} \phi_{b} u_{c} \mu_{a}^{b c}-\left(i k_{b} u_{b}+\frac{d \mathrm{v}_{\mathrm{b}}}{\mathrm{dy}}\right) u_{c} \theta_{a}^{b c}+P C \\
\left(u_{b} i k_{c} \mathrm{v}_{c}+\mathrm{v}_{\mathrm{b}} \frac{d \mathrm{v}_{c}}{d y}\right) \alpha_{a}^{b c}+i \varpi_{b} \phi_{b} \mathrm{v}_{c} \mu_{a}^{b c}-\left(i k_{b} u_{b}+\frac{d \mathrm{v}_{\mathrm{b}}}{\mathrm{dy}}\right) \mathrm{v}_{c} \theta_{a}^{b c}+P C \\
\left(u_{b} i k_{c} \phi_{c}+\mathrm{v}_{\mathrm{b}} \frac{d \phi_{c}}{d y}\right) \vartheta_{a}^{b c}+i \varpi_{b} \phi_{b} \phi_{c} \Psi_{a}^{b c}-\left(i k_{b} u_{b}+\frac{d \mathrm{v}_{\mathrm{b}}}{\mathrm{dy}}\right) \phi_{c} S_{a}^{b c}+P C
\end{array}\right]
$$

onde, assim como em (2.87), (2.88), (2.89) e em (2.90), o termo PC indica os mesmos termos à esquerda mas com a permutação dos índices b e c. Em (2.93) acima, $\alpha_{a}^{b c}, \mu_{a}^{b c}$, $\theta_{a}^{b c}, \vartheta_{a}^{b c}, \Psi_{a}^{b c} \mathrm{e} \varsigma_{a}^{b c}$ representam os coeficientes de interação entre as autofunções na vertical associadas aos modos $\mathbf{a}, \mathbf{b}$ e $\mathbf{c}$ e são dados por

$$
\begin{aligned}
& \alpha_{a}^{b c}=\frac{1}{\left\|G_{a}\right\|^{2}} \int_{\widetilde{p}_{T}}^{1} G_{b}(p) G_{c}(p) G_{a}(p) d p \quad, \quad \mu_{a}^{b c}=\frac{1}{\left\|G_{a}\right\|^{2}} \int_{\widetilde{p}_{T}}^{1} \widetilde{\widetilde{\rho}}_{T} G_{b}\left(\widetilde{p}_{T}\right) \frac{d G_{c}}{d p} G_{a}(p) d p \\
& \theta_{a}^{b c}=\frac{1}{\left\|G_{a}\right\|^{2}} \int_{\widetilde{p}_{T}}^{1} \frac{d G_{c}}{d p}\left(\int_{\widetilde{p}_{T}}^{p} G_{b}(p) d p\right) G_{a}(p) d p \quad, \quad \vartheta_{a}^{b c}=\frac{1}{\left\|G_{a}\right\|^{2}} \int_{\widetilde{p}_{T} L}^{1}\left[G_{b} \frac{G_{c}}{c_{c}{ }^{2}}-\frac{1}{\widetilde{\widetilde{\sigma}}} \frac{d G_{b}}{d p} \frac{d G_{c}}{d p}\right] G_{a}(p) d p
\end{aligned}
$$

$$
\begin{aligned}
& \Psi_{a}^{b c}=\frac{1}{\left\|G_{a}\right\|^{2}} \int_{\widetilde{p}_{T}}^{1}\left[\frac{1}{c_{c}{ }^{2}} \frac{d G_{c}}{d p}+\frac{(1-\kappa)}{p^{2} \widetilde{\widetilde{\sigma}}} \frac{d G_{c}}{d p}+\frac{(1-\kappa)}{p} \frac{G_{c}}{c_{c}{ }^{2}}\right] \widetilde{\bar{\rho}}_{T} G_{b}\left(\widetilde{p}_{T}\right) G_{a}(p) d p \\
& \varsigma_{a}^{b c}=\frac{1}{\left\|G_{a}\right\|^{2}} \int_{\widetilde{p}_{r}}^{1}\left[\frac{G_{c}}{c_{c}{ }^{2}} G_{b}+\frac{1}{c_{c}{ }^{2}} \frac{d G_{c}}{d p} \int_{\widetilde{p}_{T}}^{p} G_{b} d p-\frac{(1-\kappa)}{p \widetilde{\sigma}} \frac{d G_{c}}{d p} G_{b}+\frac{(1-\kappa)}{p^{2} \widetilde{\tilde{\sigma}}} \frac{d G_{c}}{d p} \int_{\widetilde{p}_{T}}^{p} G_{b} d p+\frac{(1-\kappa)}{p} \frac{G_{c}}{c_{c}{ }^{2}} \int_{\widetilde{p}_{T}}^{p} G_{b} d p\right] G_{a}(p) d p
\end{aligned}
$$


sendo que $\left\|\mathrm{G}_{\mathrm{a}}\right\|$ refere-se à norma da autofunção $\mathrm{G}_{\mathrm{a}}(\mathrm{p})$ dada por (2.40). Na equação (2.92) o coeficiente $\delta_{a b c}$ indica a condição de interação e é dado por:

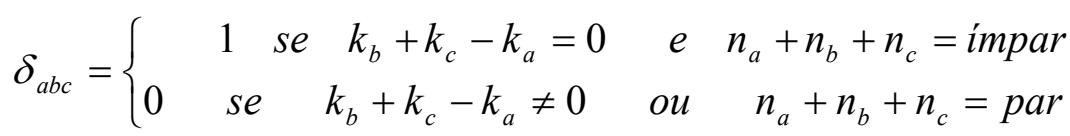

A condição de ressonância em $\mathrm{x}$ acima, dada por $\mathrm{k}_{\mathrm{a}}=\mathrm{k}_{\mathrm{b}}+\mathrm{k}_{\mathrm{c}}$, resulta da ortogonalidade das funções trigonométricas complexas que definem a estrutura em x. Já a condição de ressonância em y acima resulta do fato de que, devido às propriedades de paridade e imparidade das funções de Hermite discutidas anteriormente, a condição que $\mathrm{n}_{\mathrm{a}}+$ $\mathrm{n}_{\mathrm{b}}+\mathrm{n}_{\mathrm{c}}$ seja ímpar num determinado tripleto $\mathbf{a}, \mathbf{b}$ e c é necessária para que os integrandos em (2.92), que são constituídos por produtos de três funções de Hermite, sejam funções pares com relação à y. Esta condição, juntamente com as condições $\varpi_{\mathrm{a}}=\varpi_{\mathrm{b}}+\varpi_{\mathrm{c}} \mathrm{e} \mathrm{k}_{\mathrm{a}}=\mathrm{k}_{\mathrm{b}}+\mathrm{k}_{\mathrm{c}}$, constituem as condições de ressonância associadas à geometria do plano $\beta$-equatorial. A determinação de possíveis tripletos que satisfazem tais condições será discutida no Capítulo 4. É importante observar que a condição de ressonância em y, dada por $\mathrm{n}_{\mathrm{a}}+\mathrm{n}_{\mathrm{b}}+\mathrm{n}_{\mathrm{c}}$ ímpar, é menos restritiva que a condição que impõe que a soma dos números de onda meridionais seja nula, como é o caso da geometria do plano- $\beta$ de médias latitudes (Duffy 1974). Isto sugere que a existência de interações não lineares ressonantes seja mais favorecida quanto maior a existência de não-homogeneidades no domínio, que se reflete no aumento do número de coeficientes variáveis no sistema de equações diferenciais parciais que representa um dado modelo atmosférico. Logo, extrapolando para a atmosfera real, que é caracterizada pela existência de diversas não-homogeneidades, tais como a geometria esférica, topografia, gradientes térmicos horizontais decorrentes do contraste entre continentes e oceanos e etc, é provável que as trocas de energia entre ondas ressonantes sejam muito mais favorecidas que o sugerido em modelos teóricos.

A partir das equações (2.92) a (2.94) nota-se que as interações não lineares se dão, na mais baixa ordem, por meio de tríades (interações triádicas) nas quais os coeficientes de acoplamento $\eta_{a}^{b c}$ representam o grau de acoplamento intrínseco entre três modos quaisquer que constituem a solução de ordem dominante do modelo. Estes coeficientes indicam o quanto o produto entre dois modos $\mathbf{b}$ e $\mathbf{c}$ quaisquer devido à contribuição de ordem dominante dos termos não lineares das equações governantes pode ser projetado no modo a. Assim, na mais baixa ordem, toda a informação da não linearidade envolvendo as ondas permitidas pelo 
modelo está contida nesses coeficientes. A partir das equações (2.92) e (2.93) segue que esses coeficientes de acoplamento são invariantes com relação à permutação dos sobre-índices $\mathbf{b}$ e c. Com a normalização das autofunções $\xi_{\mathrm{a}}(\mathrm{y})$ em (2.45), (2.50) e (2.51), com as componentes $\mathrm{u}_{\mathrm{a}}$ e $\phi_{\mathrm{a}}$ como reais e $\mathrm{v}_{\mathrm{a}}$ como imaginário puro, segue que os coeficientes de acoplamento $\eta_{a}^{b c}$ são números imaginários puros. No caso de uma única tríade ressonante, a equação (2.91) reduz-se ao seguinte sistema de três equações a três incógnitas para as amplitudes dos modos $a, b$ e c:

$$
\begin{aligned}
& c_{a}{ }^{2} \frac{d A_{a}}{d \tau}=A_{b} A_{c} \eta_{a}^{b c} \\
& c_{b}{ }^{2} \frac{d A_{b}}{d \tau}=A_{a} A_{c}^{*} \eta_{b}^{a c} \\
& c_{c}{ }^{2} \frac{d A_{c}}{d \tau}=A_{a} A_{b}^{*} \eta_{c}^{a b}
\end{aligned}
$$

Dessa forma, a partir de (2.91) ou (2.96) observa-se que a condição de solvabilidade (2.72) no caso ressonante leva ao acoplamento das amplitudes de diferentes modos na escala longa de tempo por meio de interações triádicas. Logo, a evolução da amplitude das ondas na escala longa de tempo representada por (2.96) devido às interações triádicas assegura a eliminação das soluções seculares e, conseqüentemente, a validade das expansões assintóticas dadas por (2.22). Entretanto, uma dificuldade adicional surgiria se a amplitude $\mathrm{A}_{\mathrm{a}}$ de algum modo qualquer apresentasse um crescimento ilimitado em $\tau$. De acordo com as equações (2.96), isto acontece somente se os coeficientes de interação $\eta_{\mathrm{a}}{ }^{\mathrm{bc}}, \eta_{\mathrm{b}}{ }^{\mathrm{ac}}$ e $\eta_{\mathrm{c}}{ }^{\mathrm{ab}}$ numa determinada tríade ressonante possuírem o mesmo sinal. Neste caso, as amplitudes $A_{a}, A_{b} e$ $\mathrm{A}_{\mathrm{c}}$ associadas aos modos $\mathbf{a}, \mathbf{b}$ e $\mathbf{c}$, respectivamente, crescem ilimitadamente em $\tau$, apresentando singularidade em tempo finito. Tríades ressonantes que apresentam tal característica são denominadas tríades explosivas. Este comportamento, obviamente, seria inaceitável do ponto de vista de uma teoria de perturbação de amplitude finita. O método comumente empregado para solucionar este problema e, conseqüentemente, garantir a uniformidade da expansão assintótica dada por (2.22), consiste em incluir em (2.96) efeitos de ordem maior não considerados no problema de $\mathrm{O}(\varepsilon)$, como por exemplo os termos não lineares cúbicos de ordem $\varepsilon^{2}$ em (2.19). Conforme mostrado em Mahoney (1994) para um problema qualquer que admite tríades ressonantes explosivas e em Weiland e Wilhelmsson 
(1977) para um problema de física de plasmas, a inclusão de termos de ordem mais alta tem como efeito estabilizar a solução das equações do tipo (2.96) por meio de repetidas saturações das explosões da solução em $\tau$.

Todavia, no problema aqui abordado, além dos coeficientes de interação $\eta_{\mathrm{a}}{ }^{\mathrm{bc}}, \eta_{\mathrm{b}}{ }^{\mathrm{ac}} \mathrm{e}$ $\eta_{\mathrm{c}}^{\mathrm{ab}}$ para os tripletos ressonantes determinados no Capítulo 4 serem imaginários puros e possuírem sinais diferentes, estes tripletos conservam o termo de ordem dominante da energia total do modelo representado pelas equações (2.1) e com as condições de fronteira na vertical dadas por (2.9), como será mostrado nos capítulos seguintes, garantindo, assim, a regularidade da solução em $\tau$ e, conseqüentemente, a uniformidade das expansões assintóticas dadas por (2.22), não havendo a necessidade da inclusão de termos de ordem mais alta. A existência de tríades ressonantes explosivas em problemas relacionados com mecânica de fluidos geofísicos é possível somente na presença de um campo básico cisalhante e que apresenta uma camada crítica, como será discutido no Capítulo 6. 


\section{CAPÍTULO 3}

\section{Algumas Relações Energéticas Envolvendo as Ondas Equatoriais}

No capítulo anterior foi obtido a partir da versão adiabática das equações primitivas em coordenadas isobáricas um modelo assintótico reduzido que governa a evolução fracamente não linear da amplitude das ondas numa determinada tríade ressonante. Este modelo reduzido foi obtido a partir da aplicação da teoria clássica de perturbação assintótica e do método de múltiplas escalas temporais nas equações governantes na forma adimensional. No contexto de qualquer movimento ondulatório, é perfeitamente conhecido e até mesmo intuitivo que a amplitude de uma determinada onda está, de alguma forma, diretamente relacionada com sua energia. Logo, as equações (2.96) descrevem a transferência de energia entre ondas numa determinada tríade ressonante. Assim, o presente capítulo tem como objetivo determinar o princípio da conservação da energia no modelo atmosférico aqui adotado e, com isso, obter relações gerais de energia envolvendo as ondas que interagem num determinado tripleto ressonante. Este tipo de análise é importante pois, sabendo o princípio geral da conservação da energia do modelo de equações original e o aplicando para as ondas obtidas como solução de ordem dominante desse modelo, é possível obter vínculos que os coeficientes de interação devem satisfazer para garantir tal lei de conservação. Tais vínculos, por sua vez, estabelecem preciosas informações sobre a estabilidade das ondas e também quais ondas são mais energeticamente ativas num determinado tripleto ressonante.

Assim, multiplicando as equações (2.18a) e (2.18b) por u e v, respectivamente, e somando as duas equações resultantes, obtém-se:

$$
\frac{\partial}{\partial t}\left(\frac{u^{2}+\mathrm{v}^{2}}{2}\right)+\varepsilon\left[\vec{V} \bullet \nabla\left(\frac{u^{2}+\mathrm{v}^{2}}{2}\right)+F \omega \frac{\partial}{\partial p}\left(\frac{u^{2}+\mathrm{v}^{2}}{2}\right)\right]+\vec{V} \bullet \nabla \phi=0
$$

onde $\vec{V}=(u, \mathrm{v})^{\mathrm{T}}$ representa o campo vetorial associado ao vento horizontal e o operador $\nabla=$ $\left(\frac{\partial}{\partial x}, \frac{\partial}{\partial y}\right)^{T}$, sendo as derivadas em $\mathrm{x}$ e $\mathrm{y}$, assim como as derivadas temporais, dadas ao longo de superfícies isobáricas. Usando a equação da continuidade (2.18c), é possível reescrever (3.1) acima na forma de fluxo como segue: 


$$
\frac{\partial}{\partial t}\left(\frac{u^{2}+\mathrm{v}^{2}}{2}\right)+\varepsilon\left\{\nabla \bullet\left[\vec{V}\left(\frac{u^{2}+\mathrm{v}^{2}}{2}\right)\right]+\frac{\partial}{\partial p}\left[F \omega\left(\frac{u^{2}+\mathrm{v}^{2}}{2}\right)\right]\right\}=-\nabla \bullet(\vec{V} \phi)-\frac{\partial}{\partial p}(F \omega \phi)+F \omega \frac{\partial \phi}{\partial p}
$$

Nas equações acima, $\frac{u^{2}+v^{2}}{2}$ corresponde à densidade de energia cinética, enquanto os termos entre chaves em (3.2) representam seus respectivos fluxos. Os termos $\nabla \bullet(\vec{V} \phi)$ e $\frac{\partial}{\partial p}(F \omega \phi)$ podem ser interpretados fisicamente como os fluxos horizontal e vertical, respectivamente, de energia potencial. Analogamente, multiplicando a equação da termodinâmica (2.18d) por $\frac{1}{\widetilde{\widetilde{\sigma}}} \frac{\partial \phi}{\partial p}$ e usando também a equação da continuidade $(2.18 \mathrm{c})$, obtém-se a seguinte equação na forma de fluxo:

$$
\frac{\partial}{\partial t}\left[\frac{1}{2 \widetilde{\widetilde{\sigma}}}\left(\frac{\partial \phi}{\partial p}\right)^{2}\right]+\varepsilon\left\{\nabla \bullet\left[\frac{\vec{V}}{2 \widetilde{\widetilde{\sigma}}}\left(\frac{\partial \phi}{\partial p}\right)^{2}\right]+\frac{\partial}{\partial p}\left[\frac{F \omega}{2 \widetilde{\widetilde{\sigma}}}\left(\frac{\partial \phi}{\partial p}\right)^{2}\right]+\frac{F \omega(1-\kappa)}{p \widetilde{\widetilde{\sigma}}}\left(\frac{\partial \phi}{\partial p}\right)^{2}\right\}=-F \omega \frac{\partial \phi}{\partial p}
$$

Assim, somando as equações (3.2) e (3.3), o termo quadrático de conversão no lado direito dessas duas equações desaparece e a seguinte equação é obtida para a densidade de energia total:

$$
\begin{aligned}
& \frac{\partial}{\partial t}\left[\frac{u^{2}+\mathrm{v}^{2}}{2}+\frac{1}{2 \widetilde{\widetilde{\sigma}}}\left(\frac{\partial \phi}{\partial p}\right)^{2}\right]+\varepsilon\left\{\nabla \bullet\left[\vec{V}\left(\frac{u^{2}+\mathrm{v}^{2}}{2}+\frac{1}{2 \widetilde{\widetilde{\sigma}}}\left(\frac{\partial \phi}{\partial p}\right)^{2}\right)\right]+\frac{\partial}{\partial p}\left[F \omega\left(\frac{u^{2}+\mathrm{v}^{2}}{2}+\frac{1}{2 \widetilde{\widetilde{\sigma}}}\left(\frac{\partial \phi}{\partial p}\right)^{2}\right)\right]\right\} \\
& +\varepsilon \frac{F \omega(1-\kappa)}{p \widetilde{\sigma}}\left(\frac{\partial \phi}{\partial p}\right)^{2}+\nabla \bullet(\vec{V} \phi)+\frac{\partial(F \omega \phi)}{\partial p}=0
\end{aligned}
$$

Dado que o estado básico aqui considerado é caracterizado pela ausência de movimento, ou seja, $\overline{\mathrm{u}}=\overline{\mathrm{v}}=\bar{\omega}=\overline{\mathrm{w}}=0$, segue que o termo $\frac{1}{2 \widetilde{\sigma}}\left(\frac{\partial \phi}{\partial p}\right)^{2}$ pode ser fisicamente interpretado como a densidade de energia potencial disponível. Substituindo u, v, 
$\phi$ e $\omega$ na equação da energia (3.4) por suas respectivas contribuições de ordem dominante dadas por $\mathrm{u}^{(0)}, \mathrm{v}^{(0)}, \phi^{(0)} \mathrm{e} \omega^{(0)}$ e integrando a equação resultante ao longo do domínio [- $\left.\widetilde{L}_{x}, \widetilde{L}_{x}\right]$ $\mathrm{X}(-\infty,+\infty) \mathrm{X}\left[\tilde{p}_{T}, 1\right]$, obtém-se:

$$
\begin{aligned}
& \frac{\partial}{\partial t} \int_{-\widetilde{L}_{x}}^{\widetilde{L}_{x}} \int_{-\infty}^{+\infty} \int_{\widetilde{p}_{T}}^{1}\left[\frac{u^{(0)^{2}}+\mathrm{v}^{(0)^{2}}}{2}+\frac{1}{2 \widetilde{\widetilde{\sigma}}}\left(\frac{\partial \phi^{(0)}}{\partial p}\right)^{2}\right] d p d y d x+ \\
& \varepsilon \int_{-\widetilde{L}_{x}}^{\widetilde{L}_{x}} \int_{-\infty}^{+\infty}\left\{\int_{\widetilde{p}_{T}}^{1} \nabla \bullet\left[\vec{V}^{(0)}\left(\frac{u^{(0)^{2}}+\mathrm{v}^{(0)^{2}}}{2}+\frac{1}{2 \widetilde{\sigma}}\left(\frac{\partial \phi^{(0)}}{\partial p}\right)^{2}\right)\right] d p+\left[\left.F \omega^{(0)}\left(\frac{u^{(0)^{2}}+\mathrm{v}^{(0)^{2}}}{2}+\frac{1}{2 \widetilde{\sigma}}\left(\frac{\partial \phi^{(0)}}{\partial p}\right)^{2}\right)\right|_{\widetilde{p}_{T}} ^{1}\right\} d y d x\right. \\
& +\varepsilon \int_{-\widetilde{L}_{x}}^{\widetilde{L}_{x}} \int_{-\infty}^{+\infty} \int_{\widetilde{p}_{T}}^{1} \frac{F \omega^{(0)}(1-\kappa)}{p \widetilde{\bar{\sigma}}}\left(\frac{\partial \phi^{(0)}}{\partial p}\right)^{2} d p d y d x+\int_{-\widetilde{L}_{x}}^{\widetilde{L}_{-\infty}} \int_{\widetilde{p}_{T}}^{+\infty} \nabla \bullet\left(\vec{V}^{(0)} \phi^{(0)}\right) d p d y d x+\left.\int_{-\widetilde{L}_{x}}^{\widetilde{L}_{x}} \int_{-\infty}^{+\infty} F \omega^{(0)} \phi^{(0)}\right|_{\widetilde{p}_{T}} ^{1} d y d x=0
\end{aligned}
$$

Usando o Teorema de Gauss ou da Divergência e as condições de fronteira na horizontal dadas por (2.10), as integrais referentes aos fluxos horizontais se anulam e, conseqüentemente, a equação da energia total pode ser escrita da seguinte forma:

$$
\frac{\partial}{\partial t} \int_{-\widetilde{L}_{x}}^{\widetilde{L}_{x}} \int_{-\infty}^{+\infty} \int_{\widetilde{p}_{T}}^{1}\left[\frac{u^{(0)^{2}}+\mathrm{v}^{(0)^{2}}}{2}+\frac{1}{2 \widetilde{\bar{\sigma}}}\left(\frac{\partial \phi^{(0)}}{\partial p}\right)^{2}\right] d p d y d x+\left.\int_{-\widetilde{L}_{x}}^{\widetilde{L}_{x}} \int_{-\infty}^{+\infty} F \omega^{(0)} \phi^{(0)}\right|_{\widetilde{p}_{T}} ^{1} d y d x+O(\varepsilon)=0
$$

Usando as condições de fronteira na vertical do problema de $\mathrm{O}(1)$ dadas por (2.26), segue que a conservação da energia total do modelo de equações primitivas pode ser escrita da seguinte forma:

$$
\frac{\partial}{\partial t} \int_{\widetilde{L}_{x}}^{\widetilde{L}_{x}} \int_{-\infty}^{+\infty}\left\{\int_{\widetilde{p}_{T}}^{1}\left[\frac{u^{(0)^{2}}+\mathrm{v}^{(0)^{2}}}{2}+\frac{1}{2 \widetilde{\widetilde{\sigma}}}\left(\frac{\partial \phi^{(0)}}{\partial p}\right)^{2}\right] d p+\left.\frac{\widetilde{\bar{\rho}} \phi^{(0)^{2}}}{2}\right|_{\widetilde{p}_{T}} ^{1}\right\} d y d x+O(\varepsilon)=0
$$

Assim, de acordo com (3.7), o termo de ordem dominante da energia total do modelo de equações primitivas adotado no presente trabalho é dado por 


$$
E^{(0)}=\int_{-\widetilde{L}_{x}}^{\widetilde{L}_{x}} \int_{-\infty}^{+\infty}\left\{\int_{\widetilde{p}_{T}}^{1}\left[\frac{u^{(0)^{2}}+\mathrm{v}^{(0)^{2}}}{2}+\frac{1}{2 \widetilde{\widetilde{\sigma}}}\left(\frac{\partial \phi^{(0)}}{\partial p}\right)^{2}\right] d p+\left.\frac{\widetilde{\bar{\rho}} \phi^{(0)^{2}}}{2}\right|_{\widetilde{p}_{T}} ^{1}\right\} d y d x
$$

Escrevendo $\mathrm{u}^{(0)}, \mathrm{v}^{(0)} \mathrm{e} \phi^{(0)} \mathrm{em}$ (3.8) em termos das auto-soluções do problema de $\mathrm{O}(1)$, ou seja, substituindo (2.53) em (3.8), é possível reescrever o termo de ordem dominante da energia total da seguinte forma:

$$
E^{(0)}=\frac{1}{2} \sum_{a} \sum_{b} A_{a} A_{b}^{*} \delta_{k_{a} k_{b}} \int_{-\infty}^{+\infty}\left[\left(u_{a} u_{b}+\mathrm{v}_{\mathrm{a}} \mathrm{v}_{b}^{*}\right) \int_{\widetilde{p}_{T}}^{1} G_{a} G_{b} d p+\phi_{a} \phi_{b} \int_{\widetilde{p}_{T}}^{1} \frac{1}{\widetilde{\bar{\sigma}}} \frac{d G_{a}}{d p} \frac{d G_{b}}{d p} d p+\phi_{a} \phi_{b} G_{a} G_{b} \widetilde{\bar{\rho}}_{\widetilde{p}_{T}}^{1}\right] d y
$$

onde $\delta_{k_{a} k_{b}}$ (função delta de Kronecher) resulta da ortogonalidade das funções trigonométricas que definem a estrutura em $\mathrm{x}$ das autofunções. Integrando por partes o segundo termo entre colchetes segue que

$$
E^{(0)}=\frac{1}{2} \sum_{a} \sum_{b} A_{a} A_{b}^{*} \delta_{k_{a} k_{b}} \int_{-\infty}^{+\infty}\left[\left(u_{a} u_{b}+\mathrm{v}_{\mathrm{a}} \mathrm{v}_{b}^{*}\right)_{\widetilde{p}_{T}}^{1} \int_{a} G_{a} G_{b} d p+\left.\phi_{a} \phi_{b} G_{a} \frac{1}{\widetilde{\bar{\sigma}}} \frac{d G_{b}}{d p}\right|_{\widetilde{p}_{T}} ^{1}-\phi_{a} \phi_{b} \int_{\widetilde{p}_{T}}^{1} G_{a} \frac{1}{\widetilde{\bar{\sigma}}} \frac{d^{2} G_{b}}{d p^{2}} d p+\phi_{a} \phi_{b} G_{a} G_{b} \widetilde{\bar{\rho}}_{\widetilde{p}_{T}}^{1}\right] d y
$$

Usando as condições de contorno (2.33) das autofunções na vertical no segundo termo entre colchetes e a própria equação (2.31) da estrutura vertical da solução do problema de $\mathrm{O}(1)$ no terceiro termo, obtém-se

$$
E^{(0)}=\frac{1}{2} \sum_{a} \sum_{b} A_{a} A_{b}^{*} \delta_{k_{a} k_{b}} \int_{-\infty}^{+\infty}\left[\left(u_{a} u_{b}+\mathrm{v}_{\mathrm{a}} \mathrm{v}_{b}^{*}+\frac{\phi_{a} \phi_{b}}{c_{b}^{2}}\right) \int_{\widetilde{p}_{T}}^{1} G_{a} G_{b} d p\right] d y
$$

Finalmente, usando a expressão para as autofunções $u_{\mathrm{a}}, \mathrm{v}_{\mathrm{a}}$ e $\phi_{\mathrm{a}}$ dada por (2.45), (2.50) ou (2.51), bem como a ortogonalidade das autofunções $\mathrm{G}_{\mathrm{a}}(\mathrm{p})$ no intervalo $\left[\tilde{p}_{T}, 1\right]$, segue que a componente de ordem dominante da energia total pode ser representada de forma diagonalizada em termos dos modos normais, sendo escrita como 


$$
E^{(0)}=\sum_{a} c_{a}{ }^{2} A_{a} A_{a}^{*}=\sum_{a} c_{a}{ }^{2}\left|A_{a}\right|^{2}
$$

Dessa forma, considerando novamente o sistema de equações (2.96) para uma única tríade ressonante, multiplicando as equações (2.96a), (2.96b) e (2.96c) por $\mathrm{A}_{\mathrm{a}}{ }^{*}, \mathrm{~A}_{\mathrm{b}}{ }^{*}$ e $\mathrm{A}_{\mathrm{c}}{ }^{*}$, respectivamente, obtém-se a equação da evolução na escala longa de tempo da energia dos modos a, b e c numa dada tríade ressonante qualquer:

$$
\begin{aligned}
& \frac{d E_{a}}{d \tau}=-i \eta_{a}^{b c} \operatorname{Im}\left(A_{a} A_{b} A_{c}\right) \\
& \frac{d E_{b}}{d \tau}=-i \eta_{b}^{a c} \operatorname{Im}\left(A_{a} A_{b} A_{c}\right) \\
& \frac{d E_{c}}{d \tau}=-i \eta_{c}^{a b} \operatorname{Im}\left(A_{a} A_{b} A_{c}\right)
\end{aligned}
$$

sendo que $E_{j}=\frac{1}{2} c_{j}{ }^{2}\left|A_{j}\right|^{2}$ representa a energia do modo $\mathbf{j}$, sendo $\mathrm{j}=\mathrm{a}, \mathrm{b}$ ou c, e $\operatorname{Im}()$ denota a parte imaginária do número complexo. Assim, uma vez que $\mathrm{c}_{\mathrm{j}}{ }^{2}>0$, de (3.13) segue que o termo de ordem dominante da energia total $\mathrm{E}^{(0)}$ é conservado se, e, somente se, os coeficientes de interação num tripleto ressonante qualquer satisfazem à seguinte relação

$$
\eta_{a}^{b c}+\eta_{b}^{a c}+\eta_{c}^{a b}=0
$$

A equação (3.14) acima tem algumas conseqüências interessantes para a dinâmica dos tripletos ressonantes. A equação (3.14) implica que, numa tríade ressonante qualquer, o coeficiente de interação com maior valor absoluto deve, necessariamente, possuir sinal oposto ao dos outros dois. A partir de (3.13) pode ser verificado que, fisicamente, esta condição significa que o modo que apresenta o coeficiente de interação com sinal oposto (e, conseqüentemente, com maior valor absoluto) sempre age como uma fonte ou sumidouro de energia para os outros dois membros da tríade ressonante, ou seja, sempre recebe energia das outras duas ondas ou cede energia para as mesmas.

Uma vez que os modos $\mathbf{a}, \mathbf{b}$ e $\mathbf{c}$ na tríade ressonante acima satisfazem necessariamente à condição de ressonância para suas freqüências temporais, dada por $\varpi_{a}=\varpi_{b}+\varpi_{c}$, pode ser notado que a condição (3.14) juntamente com a condição de ressonância implicam que, numa 
tríade ressonante qualquer, o modo com maior freqüência absoluta é sempre o modo instável, ou seja, o modo que possui o coeficiente de interação com sinal oposto ao dos outros dois. Dessa forma, assim como ocorre no modelo de água-rasa e em diversos outros problemas físicos, no modelo de equações primitivas adotado no presente trabalho o modo com maior freqüência absoluta numa determinada tríade ressonante é sempre o modo mais "energeticamente ativo"no tripleto, ou seja, sempre age como fonte ou sumidouro de energia para os outros dois membros da tríade. 


\section{CAPÍTULO 4}

\section{Determinação de possíveis tripletos ressonantes}

Como mostrado no Capítulo 2, a partir da condição de solvabilidade do problema de $\mathrm{O}(\varepsilon)$ segue que a amplitude das ondas numa determinada tríade evolui na escala longa de tempo de acordo com as equações (2.96), desde que a condição de ressonância para as freqüências temporais, dada por $\varpi_{\mathrm{a}}=\varpi_{\mathrm{b}}+\varpi_{\mathrm{c}}$, seja satisfeita. A ortogonalidade das funções trigonométricas que definem a estrutura em x das auto-soluções do problema de $\mathrm{O}(1)$ e as propriedades de paridade e imparidade das funções de Hermite que definem a dependência em y das mesmas implicam que somente os modos $\mathbf{a}, \mathbf{b}$ e c cujos números de onda zonal e modos meridionais satisfazem às relações $\mathrm{k}_{\mathrm{a}}=\mathrm{k}_{\mathrm{b}}+\mathrm{k}_{\mathrm{c}} \mathrm{e} \mathrm{n}_{\mathrm{a}}+\mathrm{n}_{\mathrm{b}}+\mathrm{n}_{\mathrm{c}}=$ ímpar, respectivamente, podem interagir entre si. Logo, o objetivo do presente capítulo consiste em determinar algumas possíveis tríades de ondas que satisfazem tais condições de ressonância para as freqüências temporais, para os números de onda zonal e para os modos meridionais. A determinação desses tripletos ressonantes requer, portanto, a solução do seguinte sistema algébrico:

$$
\begin{aligned}
& \varpi_{\mathrm{a}}=\varpi_{\mathrm{b}}+\varpi_{\mathrm{c}} \\
& \mathrm{k}_{\mathrm{a}}=\mathrm{k}_{\mathrm{b}}+\mathrm{k}_{\mathrm{c}} \\
& \mathrm{n}_{\mathrm{a}}+\mathrm{n}_{\mathrm{b}}+\mathrm{n}_{\mathrm{c}}=\text { ímpar }
\end{aligned}
$$

sendo que $\varpi_{\mathrm{j}}, \mathrm{j}=\mathrm{a}$, b ou c, satisfazem à relação de dispersão dada por (2.43). Entretanto, antes de determinar as possíveis tríades que satisfazem às condições (4.1), é necessário conhecer primeiramente as condições de ressonância impostas pelas autofunções da estrutura vertical associadas às auto-soluções do problema de $\mathrm{O}(1)$. De acordo com as equações (2.92) e (2.93), o acoplamento entre os modos $\mathbf{a}, \mathbf{b}$ e $\mathbf{c}$ numa tríade ressonante por meio de suas estruturas verticais se dá através dos coeficientes $\alpha_{\mathrm{a}}^{\mathrm{bc}}, \mu_{\mathrm{a}}^{\mathrm{bc}}, \theta_{a}^{b c}, \Psi_{a}^{b c}, \vartheta_{a}^{b c} \mathrm{e} \varsigma_{a}^{b c}$ dados por (2.94), além dos termos de acoplamento nas fronteiras dados por $-\left.\left(\frac{\widetilde{\bar{\rho}}}{c_{b}{ }^{2}}+\widetilde{\widetilde{\Gamma}}\right) \frac{G_{a}(p) G_{b}(p) G_{c}(p)}{\left\|G_{a}\right\|^{2}}\right|_{1} ^{\widetilde{p}_{T}} \mathrm{e}$ 
$\left.\Lambda \widetilde{\bar{\rho}} \frac{G_{a}(p) G_{b}(p) G_{c}(p)}{\left\|G_{a}\right\|^{2}}\right|_{1} ^{\widetilde{p}_{T}}$. Logo, para conhecer as condições de ressonância decorrentes da estrutura vertical dos modos é necessário calcular esses coeficientes. As Tabelas 4.1, 4.2, 4.3 e 4.4 mostram, respectivamente, os valores dos coeficientes $\alpha_{\mathrm{a}}^{\mathrm{bc}}, \theta_{a}^{b c}, \vartheta_{a}^{b c}$ e $\varsigma_{a}^{b c}$ para os 5 primeiros modos verticais, utilizando o perfil de $\bar{\rho}(\mathrm{p})$ mostrado na Figura 2.1 e $\mathrm{p}_{0}=1000 \mathrm{hPa}$, $\mathrm{p}_{\mathrm{T}}=100 \mathrm{hPa}\left(\widetilde{p}_{T}=0.1\right)$ e $\bar{\sigma}=2 \times 10^{-6} \mathrm{~m}^{4} \mathrm{~s}^{2} \mathrm{Kg}^{-2}(\tilde{\bar{\sigma}}=37,8072)$. Os valores dos coeficientes $\mu_{\mathrm{a}}^{\mathrm{bc}}$ e $\Psi_{a}^{b c}$ não são mostrados pois, para todos os modos verticais envolvidos, estes coeficientes são menores que $10^{-2} \mathrm{e}$, portanto, muito pequenos comparados com os demais coeficientes de acoplamento entre os modos verticais. Para o cálculo dos coeficientes $\alpha_{\mathrm{a}}{ }^{\mathrm{bc}}$, $\theta_{a}^{b c}, \vartheta_{a}^{b c}, \varsigma_{a}^{b c}, \mu_{\mathrm{a}}^{\mathrm{bc}} \mathrm{e} \Psi_{a}^{b c}$ utilizou-se o método de integração numérica do trapézio. As funções dadas por $\frac{d G_{m}}{d p}$ e $\int_{\widetilde{p}_{T}}^{p} G_{m}(p) d p$ envolvidas nesses cálculos foram obtidas analiticamente a partir da expressão (2.37), ou seja,

$$
\begin{gathered}
\frac{d G_{m}}{d p}=-\lambda_{m} \operatorname{sen}\left(\lambda_{m} p\right)+\gamma_{m} \lambda_{m} \cos \left(\lambda_{m} p\right) \\
\int_{\widetilde{p}_{T}}^{p} G_{m}(p) d p=\frac{1}{\lambda_{m}}\left[\operatorname{sen}\left(\lambda_{m} p\right)-\operatorname{sen}\left(\lambda_{m} \widetilde{p}_{T}\right)+\gamma_{m} \cos \left(\lambda_{m} \widetilde{p}_{T}\right)-\gamma_{m} \cos \left(\lambda_{m} p\right)\right]
\end{gathered}
$$

Tabela 4.1: Valores de $\alpha_{\mathrm{m}}{ }^{\mathrm{j} 1}$ obtidos por (2.94) para: (a) $\mathrm{m}=0$, (b) $\mathrm{m}=1$, (c) $\mathrm{m}=2$, (d) $\mathrm{m}=3$ e (e) $\mathrm{m}=4$.

(a)

\begin{tabular}{|c|l|l|l|l|l|l|}
\hline \multicolumn{7}{|c|}{$\mathrm{m}=0$} \\
\hline $\mathrm{j}$ & \multicolumn{1}{|c|}{0} & \multicolumn{1}{c|}{1} & \multicolumn{1}{c|}{2} & \multicolumn{1}{c|}{3} & \multicolumn{1}{c|}{4} \\
\hline 0 & & 1,056072563 & $3,615 \mathrm{E}-02$ & $-5,571 \mathrm{E}-03$ & $3,815 \mathrm{E}-03$ & $-1,376 \mathrm{E}-03$ \\
\hline 1 & $3,615 \mathrm{E}-02$ & 1,047619589 & $2,907 \mathrm{E}-02$ & $-5,421 \mathrm{E}-03$ & $3,846 \mathrm{E}-03$ \\
\hline 2 & $-5,571 \mathrm{E}-03$ & $2,907 \mathrm{E}-02$ & 1,051895409 & $2,711 \mathrm{E}-02$ & $-4,836 \mathrm{E}-03$ \\
\hline 3 & $3,815 \mathrm{E}-03$ & $-5,421 \mathrm{E}-03$ & $2,711 \mathrm{E}-02$ & 1,052738106 & $2,655 \mathrm{E}-02$ \\
\hline 4 & $-1,376 \mathrm{E}-03$ & $3,846 \mathrm{E}-03$ & $-4,836 \mathrm{E}-03$ & $2,655 \mathrm{E}-02$ & 1,053037056 \\
\hline
\end{tabular}


Capítulo 4: Determinação de Possíveis Tripletos Ressonantes.

(b)

\begin{tabular}{|l|l|l|l|l|l|}
\hline \multicolumn{7}{|c|}{$\mathrm{m}=1$} \\
\hline $\mathrm{j}$ & \multicolumn{1}{|c|}{0} & \multicolumn{1}{c|}{1} & \multicolumn{1}{c|}{2} & \multicolumn{1}{c|}{3} & \multicolumn{1}{c|}{4} \\
\hline 0 & $3,615 \mathrm{E}-02$ & 1,047619589 & $2,907 \mathrm{E}-02$ & $-5,421 \mathrm{E}-03$ & $3,846 \mathrm{E}-03$ \\
\hline 1 & 1,047619589 & $-7,179 \mathrm{E}-02$ & 0,744051375 & $1,883 \mathrm{E}-02$ & $-5,045 \mathrm{E}-03$ \\
\hline 2 & $2,907 \mathrm{E}-02$ & 0,744051375 & $-4,389 \mathrm{E}-02$ & 0,744540491 & $1,541 \mathrm{E}-02$ \\
\hline 3 & $-5,421 \mathrm{E}-03$ & $1,883 \mathrm{E}-02$ & 0,744540491 & $-3,973 \mathrm{E}-02$ & 0,744663554 \\
\hline 4 & $3,846 \mathrm{E}-03$ & $-5,045 \mathrm{E}-03$ & $1,541 \mathrm{E}-02$ & 0,744663554 & $-3,833 \mathrm{E}-02$ \\
\hline
\end{tabular}

(c)

\begin{tabular}{|l|l|l|l|l|l|}
\hline \multicolumn{7}{|c|}{$\mathrm{m}=2$} \\
\hline $\mathrm{j}$ & \multicolumn{1}{|c|}{0} & \multicolumn{1}{c|}{1} & \multicolumn{1}{c|}{2} & \multicolumn{1}{c|}{3} & \multicolumn{1}{c|}{4} \\
\hline 0 & $-5,571 \mathrm{E}-03$ & $2,907 \mathrm{E}-02$ & 1,051895409 & $2,711 \mathrm{E}-02$ & $-4,836 \mathrm{E}-03$ \\
\hline 1 & $2,907 \mathrm{E}-02$ & 0,744051375 & $-4,389 \mathrm{E}-02$ & 0,744540491 & $1,541 \mathrm{E}-02$ \\
\hline 2 & 1,051895409 & $-4,389 \mathrm{E}-02$ & $1,336 \mathrm{E}-02$ & $-2,027 \mathrm{E}-02$ & 0,744976395 \\
\hline 3 & $2,711 \mathrm{E}-02$ & 0,744540491 & $-2,027 \mathrm{E}-02$ & $9,227 \mathrm{E}-03$ & $-1,619 \mathrm{E}-02$ \\
\hline 4 & $-4,836 \mathrm{E}-03$ & $1,541 \mathrm{E}-02$ & 0,744976395 & $-1,619 \mathrm{E}-02$ & $8,057 \mathrm{E}-03$ \\
\hline
\end{tabular}

(d)

\begin{tabular}{|c|l|l|l|l|l|}
\hline \multicolumn{7}{|c|}{$\mathrm{m}=3$} \\
\hline $\mathrm{j}$ & \multicolumn{1}{|c|}{0} & \multicolumn{1}{c|}{1} & \multicolumn{1}{c|}{2} & \multicolumn{1}{c|}{3} & \multicolumn{1}{c|}{4} \\
\hline 0 & $3,815 \mathrm{E}-03$ & $-5,421 \mathrm{E}-03$ & $2,711 \mathrm{E}-02$ & 1,052738106 & $2,655 \mathrm{E}-02$ \\
\hline 1 & $-5,421 \mathrm{E}-03$ & $1,883 \mathrm{E}-02$ & 0,744540491 & $-3,973 \mathrm{E}-02$ & 0,744663554 \\
\hline 2 & $2,711 \mathrm{E}-02$ & 0,744540491 & $-2,027 \mathrm{E}-02$ & $9,227 \mathrm{E}-03$ & $-1,619 \mathrm{E}-02$ \\
\hline 3 & 1,052738106 & $-3,973 \mathrm{E}-02$ & $9,227 \mathrm{E}-03$ & $-8,447 \mathrm{E}-03$ & $5,732 \mathrm{E}-03$ \\
\hline 4 & $2,655 \mathrm{E}-02$ & 0,744663554 & $-1,619 \mathrm{E}-02$ & $5,732 \mathrm{E}-03$ & $-6,307 \mathrm{E}-03$ \\
\hline
\end{tabular}

(e)

\begin{tabular}{|l|l|l|l|l|l|}
\hline \multicolumn{7}{|c|}{} & \multicolumn{1}{c|}{$\mathrm{m}=4$} & \multicolumn{1}{c|}{3} \\
\hline $\mathrm{j}$ & \multicolumn{1}{|c|}{0} & \multicolumn{1}{c|}{1} & \multicolumn{1}{c|}{2} & \multicolumn{1}{c|}{3} \\
\hline 0 & $-1,377 \mathrm{E}-03$ & $3,846 \mathrm{E}-03$ & $-4,836 \mathrm{E}-03$ & $2,655 \mathrm{E}-02$ & 1,053037056 \\
\hline 1 & $3,846 \mathrm{E}-03$ & $-5,045 \mathrm{E}-03$ & $1,541 \mathrm{E}-02$ & 0,744663554 & $-3,833 \mathrm{E}-02$ \\
\hline 2 & $-4,836 \mathrm{E}-03$ & $1,541 \mathrm{E}-02$ & 0,744976395 & $-1,619 \mathrm{E}-02$ & $8,057 \mathrm{E}-03$ \\
\hline 3 & $2,655 \mathrm{E}-02$ & 0,744663554 & $-1,619 \mathrm{E}-02$ & $5,732 \mathrm{E}-03$ & $-6,307 \mathrm{E}-03$ \\
\hline 4 & 1,053037056 & $-3,833 \mathrm{E}-02$ & $8,057 \mathrm{E}-03$ & $-6,307 \mathrm{E}-03$ & $3,383 \mathrm{E}-03$ \\
\hline
\end{tabular}


Capítulo 4: Determinação de Possíveis Tripletos Ressonantes.

Tabela 4.2: Valores de $\theta_{m}^{j l}$ obtidos por (2.94) para: (a) $\mathrm{m}=0$, (b) $\mathrm{m}=1$, (c) $\mathrm{m}=2$, (d) $\mathrm{m}=3 \mathrm{e}$ (e) $\mathrm{m}=4$.

(a)

\begin{tabular}{|c|l|l|l|l|l|l|}
\hline \multicolumn{7}{|c|}{$\mathrm{m}=0$} \\
\hline $\mathrm{j}$ & 1 & 0 & 1 & \multicolumn{1}{c|}{2} & \multicolumn{1}{c|}{4} \\
\hline 0 & $-7,713 \mathrm{E}-02$ & $-1,46334318$ & 1,3928364 & $-1,387487$ & 1,38195808 \\
\hline 1 & $-3,373 \mathrm{E}-002$ & $-1,00803743$ & $-9,433 \mathrm{E}-02$ & $5,764 \mathrm{E}-02$ & $-5,511 \mathrm{E}-02$ \\
\hline 2 & $5,659 \mathrm{E}-03$ & $-2,058 \mathrm{E}-02$ & $-1,04525754$ & $-4,631 \mathrm{E}-02$ & $1,652 \mathrm{E}-02$ \\
\hline 3 & $-3,719 \mathrm{E}-03$ & $6,635 \mathrm{E}-03$ & $-2,229 \mathrm{E}-02$ & $-1,04815641$ & $-3,952 \mathrm{E}-02$ \\
\hline 4 & $1,413 \mathrm{E}-03$ & $-2,645 \mathrm{E}-03$ & $4,158 \mathrm{E}-03$ & $-2,136 \mathrm{E}-02$ & $-1,05136086$ \\
\hline
\end{tabular}

(b)

\begin{tabular}{|c|l|l|l|l|l|}
\hline \multicolumn{7}{|c|}{$\mathrm{m}=1$} \\
\hline $\mathrm{j}$ & \multicolumn{1}{|c|}{0} & \multicolumn{1}{c|}{1} & \multicolumn{1}{c|}{2} & \multicolumn{1}{c|}{3} & 4 \\
\hline 0 & $6,383 \mathrm{E}-02$ & 0,50675693 & $-2,87081484$ & 2,36363511 & $-2,23481436$ \\
\hline 1 & $7,749 \mathrm{E}-03$ & $1,175 \mathrm{E}-04$ & $-1,39549496$ & $-0,11823861$ & $8,717 \mathrm{E}-02$ \\
\hline 2 & $-1,717 \mathrm{E}-02$ & $-0,36545717$ & $3,623 \mathrm{E}-02$ & $-1,10349884$ & $-3,988 \mathrm{E}-02$ \\
\hline 3 & $4,264 \mathrm{E}-03$ & $-1,356 \mathrm{E}-02$ & $-0,48964372$ & $3,327 \mathrm{E}-02$ & $-0,98483087$ \\
\hline 4 & $-3,392 \mathrm{E}-03$ & $4,179 \mathrm{E}-03$ & $-9,66 \mathrm{E}-03$ & $-0,55640893$ & $3,62 \mathrm{E}-02$ \\
\hline
\end{tabular}

(c)

\begin{tabular}{|c|c|c|c|c|c|}
\hline \multicolumn{6}{|c|}{$\mathrm{m}=2$} \\
\hline & 0 & 1 & 2 & 3 & 4 \\
\hline 0 & $-1,278 \mathrm{E}-02$ & 0,7638006 & 0,52150217 & $-3,88347041$ & 2,81825797 \\
\hline 1 & $1,754 \mathrm{E}-02$ & 0,72358265 & $-1,442 \mathrm{E}-02$ & $-2,10468817$ & $-0,13339156$ \\
\hline 2 & $2,123 \mathrm{E}-03$ & $-5,581 \mathrm{E}-03$ & $-4,837 \mathrm{E}-06$ & $2,316 \mathrm{E}-02$ & $-1,4732005$ \\
\hline 3 & $-1,034 \mathrm{E}-02$ & $-0,24654761$ & $5,927 \mathrm{E}-03$ & $2,510 \mathrm{E}-04$ & $1,463 \mathrm{E}-02$ \\
\hline 4 & $2,887 \mathrm{E}-03$ & $-9,092 \mathrm{E}-03$ & $-0,3708042$ & $1,074 \mathrm{E}-02$ & $-5,356 \mathrm{E}-03$ \\
\hline
\end{tabular}

(d)

\begin{tabular}{|c|l|l|l|l|l|c|}
\hline \multicolumn{7}{|c|}{$\mathrm{m}=3$} \\
\hline $\mathrm{j}$ & 1 & 0 & 1 & \multicolumn{1}{c|}{2} & 3 & 4 \\
\hline 0 & $7,041 \mathrm{E}-03$ & $-0,27701334$ & 1,75818136 & 0,52436264 & $-4,93040577$ \\
\hline 1 & $-4,425 \mathrm{E}-03$ & $2,715 \mathrm{E}-002$ & 1,43298909 & $-1,661 \mathrm{E}-02$ & $-2,81336961$ \\
\hline 2 & $1,043 \mathrm{E}-02$ & 0,37222329 & $-1,626 \mathrm{E}-02$ & $2,084 \mathrm{E}-03$ & $2,526 \mathrm{E}-02$ \\
\hline 3 & $9,496 \mathrm{E}-04$ & $-1,905 \mathrm{E}-03$ & $-1,047 \mathrm{E}-03$ & $1,742 \mathrm{E}-06$ & $3,532 \mathrm{E}-03$ \\
\hline 4 & $-7,399 \mathrm{E}-03$ & $-0,18490865$ & $2,08 \mathrm{E}-03$ & $-1,177 \mathrm{E}-03$ & $5,317 \mathrm{E}-03$ \\
\hline
\end{tabular}


Capítulo 4: Determinação de Possíveis Tripletos Ressonantes.

(e)

\begin{tabular}{|c|l|l|l|l|l|}
\hline \multicolumn{7}{|c|}{$\mathrm{m}=4$} \\
\hline $\mathrm{j}$ & \multicolumn{1}{|c|}{0} & \multicolumn{1}{c|}{1} & \multicolumn{1}{c|}{2} & \multicolumn{1}{c|}{3} & 4 \\
\hline 0 & $-3,197 \mathrm{E}-03$ & 0,1486287 & $-0,71409464$ & 2,8012426 & 0,5253635 \\
\hline 1 & $3,445 \mathrm{E}-03$ & $-9,831 \mathrm{E}-03$ & $4,51 \mathrm{E}-02$ & 2,14170142 & $-1,735 \mathrm{E}-02$ \\
\hline 2 & $-2,907 \mathrm{E}-03$ & $1,12 \mathrm{E}-02$ & 0,74160458 & $-2,247 \mathrm{E}-02$ & $2,676 \mathrm{E}-03$ \\
\hline 3 & $7,439 \mathrm{E}-03$ & 0,24853411 & $-6,873 \mathrm{E}-03$ & $-8,158 \mathrm{E}-04$ & $-1,073 \mathrm{E}-03$ \\
\hline 4 & $5,382 \mathrm{E}-04$ & $-1,216 \mathrm{E}-03$ & $6,735 \mathrm{E}-04$ & $-2,39 \mathrm{E}-03$ & $-4,116 \mathrm{E}-07$ \\
\hline
\end{tabular}

Tabela 4.3: Valores de $\vartheta_{m}^{j l}$ obtidos por (2.94) para: (a) $\mathrm{m}=0$, (b) $\mathrm{m}=1$, (c) $\mathrm{m}=2$, (d) $\mathrm{m}=3 \mathrm{e}$ (e) $\mathrm{m}=4$.

(a)

\begin{tabular}{|c|l|l|l|l|l|l|}
\hline \multicolumn{7}{|c|}{$\mathrm{m}=0$} \\
\hline$j$ & \multicolumn{1}{|c|}{0} & \multicolumn{1}{c|}{1} & \multicolumn{1}{c|}{2} & \multicolumn{1}{c|}{3} & 4 \\
\hline 0 & $4,952 \mathrm{E}-03$ & $5,88 \mathrm{E}-04$ & $-9,694 \mathrm{E}-05$ & $6,46 \mathrm{E}-05$ & $-2,458 \mathrm{E}-05$ \\
\hline 1 & $-1,112 \mathrm{E}-02$ & $8,105 \mathrm{E}-03$ & $5,924 \mathrm{E}-03$ & $-1,475 \mathrm{E}-03$ & $1,155 \mathrm{E}-03$ \\
\hline 2 & $7,016 \mathrm{E}-03$ & $-2,177 \mathrm{E}-02$ & $8,209 \mathrm{E}-03$ & $1,352 \mathrm{E}-02$ & $-3,753 \mathrm{E}-03$ \\
\hline 3 & $-1,086 \mathrm{E}-02$ & $1,23 \mathrm{E}-02$ & $-2,953 \mathrm{E}-02$ & $8,229 \mathrm{E}-03$ & $2,149 \mathrm{E}-02$ \\
\hline 4 & $6,981 \mathrm{E}-03$ & $-1,717 \mathrm{E}-02$ & $1,468 \mathrm{E}-02$ & $-3,754 \mathrm{E}-02$ & $8,235 \mathrm{E}-03$ \\
\hline
\end{tabular}

(b)

\begin{tabular}{|c|l|l|l|l|l|l|}
\hline \multicolumn{7}{|c|}{$\mathrm{m}=1$} \\
\hline $\mathrm{j}$ & \multicolumn{1}{|c|}{0} & \multicolumn{1}{c|}{1} & \multicolumn{1}{c|}{2} & \multicolumn{1}{c|}{3} & 4 \\
\hline 0 & $5,881 \mathrm{E}-04$ & 0,34738193 & $1,534 \mathrm{E}-02$ & $-3,23 \mathrm{E}-03$ & $2,4 \mathrm{E}-03$ \\
\hline 1 & $8,105 \mathrm{E}-03$ & $-2,359 \mathrm{E}-02$ & 0,48535112 & $1,513 \mathrm{E}-02$ & $-4,417 \mathrm{E}-03$ \\
\hline 2 & $-2,177 \mathrm{E}-02$ & $-0,22367762$ & $-1,919 \mathrm{E}-02$ & 0,72219794 & $1,991 \mathrm{E}-02$ \\
\hline 3 & $1,23 \mathrm{E}-02$ & $-3,272 \mathrm{E}-02$ & $-0,46019333$ & $-1,854 \mathrm{E}-02$ & 0,95884472 \\
\hline 4 & $-1,717 \mathrm{E}-02$ & $1,962 \mathrm{E}-02$ & $-3,883 \mathrm{E}-02$ & $-0,696763$ & $-1,832 \mathrm{E}-02$ \\
\hline
\end{tabular}

(c)

\begin{tabular}{|c|l|l|l|l|l|l|}
\hline \multicolumn{7}{|c|}{$\mathrm{m}=2$} \\
\hline $\mathrm{j}$ & \multicolumn{1}{|c|}{0} & \multicolumn{1}{c|}{1} & \multicolumn{1}{c|}{2} & \multicolumn{1}{c|}{3} & \multicolumn{1}{c|}{4} \\
\hline 0 & $-9,683 \mathrm{E}-05$ & $1,534 \mathrm{E}-02$ & 1,35125264 & $4,813 \mathrm{E}-02$ & $-9,927 \mathrm{E}-03$ \\
\hline 1 & $5,923 \mathrm{E}-03$ & 0,48535132 & $-6,102 \mathrm{E}-02$ & 1,431693 & $3,46 \mathrm{E}-02$ \\
\hline 2 & $8,209 \mathrm{E}-03$ & $-1,919 \mathrm{E}-02$ & $1,712 \mathrm{E}-02$ & $-4,117 \mathrm{E}-02$ & 1,90579952 \\
\hline 3 & $-2,953 \mathrm{E}-02$ & $-0,46019312$ & $-8,979 \mathrm{E}-03$ & $1,45 \mathrm{E}-02$ & $-4,048 \mathrm{E}-02$ \\
\hline 4 & $1,468 \mathrm{E}-02$ & $-3,883 \mathrm{E}-02$ & $-0,93358729$ & $-4,486 \mathrm{E}-03$ & $1,376 \mathrm{E}-02$ \\
\hline
\end{tabular}


Capítulo 4: Determinação de Possíveis Tripletos Ressonantes.

(d)

\begin{tabular}{|c|l|l|l|l|l|l|}
\hline \multicolumn{7}{|c|}{$\mathrm{m}=3$} \\
\hline$j$ & 1 & \multicolumn{1}{|c|}{0} & \multicolumn{1}{c|}{1} & \multicolumn{1}{c|}{2} & \multicolumn{1}{c|}{3} & \multicolumn{1}{c|}{4} \\
\hline 0 & $6,42 \mathrm{E}-05$ & $-3,23 \mathrm{E}-03$ & $4,813 \mathrm{E}-02$ & 3,02418304 & $9,755 \mathrm{E}-02$ \\
\hline 1 & $-1,474 \mathrm{E}-03$ & $1,513 \mathrm{E}-02$ & 1,43169332 & $-0,11948983$ & 2,85104407 \\
\hline 2 & $1,352 \mathrm{E}-02$ & 0,72219849 & $-4,117 \mathrm{E}-02$ & $2,915 \mathrm{E}-02$ & $-6,619 \mathrm{E}-02$ \\
\hline 3 & $8,229 \mathrm{E}-03$ & $-1,854 \mathrm{E}-02$ & $1,45 \mathrm{E}-02$ & $-2,424 \mathrm{E}-02$ & $2,32 \mathrm{E}-02$ \\
\hline 4 & $-3,754 \mathrm{E}-02$ & $-0,69676248$ & $-4,486 \mathrm{E}-03$ & $1,046 \mathrm{E}-02$ & $-2,118 \mathrm{E}-02$ \\
\hline
\end{tabular}

(e)

\begin{tabular}{|c|l|l|l|l|l|c|}
\hline \multicolumn{7}{|c|}{$\mathrm{m}=4$} \\
\hline $\mathrm{j}$ & \multicolumn{1}{|c|}{0} & \multicolumn{1}{c|}{1} & \multicolumn{1}{c|}{2} & \multicolumn{1}{c|}{3} & 4 \\
\hline 0 & $-2,413 \mathrm{E}-05$ & $2,399 \mathrm{E}-03$ & $-9,927 \mathrm{E}-03$ & $9,755 \mathrm{E}-02$ & 5,36626 \\
\hline 1 & $1,154 \mathrm{E}-03$ & $-4,416 \mathrm{E}-03$ & $3,46 \mathrm{E}-02$ & 2,85104456 & $-0,20093233$ \\
\hline 2 & $-3,752 \mathrm{E}-03$ & $1,991 \mathrm{E}-02$ & 1,90580035 & $-6,619 \mathrm{E}-02$ & $4,447 \mathrm{E}-02$ \\
\hline 3 & $2,149 \mathrm{E}-02$ & 0,95884576 & $-4,048 \mathrm{E}-02$ & $2,32 \mathrm{E}-02$ & $-3,52 \mathrm{E}-02$ \\
\hline 4 & $8,236 \mathrm{E}-03$ & $-1,832 \mathrm{E}-02$ & $1,376 \mathrm{E}-02$ & $-2,118 \mathrm{E}-02$ & $1,723 \mathrm{E}-02$ \\
\hline
\end{tabular}

Tabela 4.4: Valores de $\varsigma_{m}^{j l}$ obtidos por (2.94) para: (a) $\mathrm{m}=0$, (b) $\mathrm{m}=1$, (c) $\mathrm{m}=2$, (d) $\mathrm{m}=3 \mathrm{e}$ (e) $\mathrm{m}=4$.

(a)

\begin{tabular}{|c|l|l|l|l|l|l|}
\hline \multicolumn{7}{|c|}{$\mathrm{m}=0$} \\
\hline $\mathrm{j}$ & \multicolumn{1}{|c|}{0} & \multicolumn{1}{c|}{1} & \multicolumn{1}{c|}{2} & \multicolumn{1}{c|}{3} & 4 \\
\hline 0 & $-9,5 \mathrm{E}-03$ & $-2,849 \mathrm{E}-05$ & $-4,377 \mathrm{E}-06$ & $-2,477 \mathrm{E}-06$ & $-1,345 \mathrm{E}-06$ \\
\hline 1 & 0,469049 & $-1,794 \mathrm{E}-02$ & $-2,958 \mathrm{E}-03$ & $-4,389 \mathrm{E}-04$ & $-4,146 \mathrm{E}-04$ \\
\hline 2 & $-1,77878418$ & $8,302 \mathrm{E}-02$ & $-1,354 \mathrm{E}-02$ & $-6,44 \mathrm{E}-03$ & $7,917 \mathrm{E}-04$ \\
\hline 3 & 3,9709887 & $-0,15020366$ & $5,452 \mathrm{E}-02$ & $-1,822 \mathrm{E}-02$ & $-1,52 \mathrm{E}-02$ \\
\hline 4 & $-7,0320111$ & 0,260814 & $-5,982 \mathrm{E}-02$ & $6,551 \mathrm{E}-02$ & $-1,362 \mathrm{E}-02$ \\
\hline
\end{tabular}

(b)

\begin{tabular}{|c|c|c|c|c|c|}
\hline \multicolumn{6}{|c|}{$\mathrm{m}=1$} \\
\hline $\mathrm{j}$ & 0 & 1 & 2 & 3 & 4 \\
\hline 0 & $-1,123 \mathrm{E}-03$ & $-1,042 \mathrm{E}-02$ & $-2,293 \mathrm{E}-04$ & $-3,431 \mathrm{E}-05$ & $-2,288 \mathrm{E}-05$ \\
\hline 1 & $-0,71588521$ & $-0,13474427$ & $-0,14794451$ & $4,418 \mathrm{E}-03$ & $1,324 \mathrm{E}-03$ \\
\hline 2 & 3,67371552 & 0,74341604 & $-0,13458008$ & $-0,35514445$ & $4,037 \mathrm{E}-03$ \\
\hline 3 & $-6,74657424$ & 0,33765458 & 0,96658319 & $-0,12163037$ & $-0,57068493$ \\
\hline 4 & 11,3800244 & $-0,40366722$ & 0,16978456 & 1,16933575 & $-0,12730361$ \\
\hline
\end{tabular}


(c)

\begin{tabular}{|c|l|l|l|l|l|l|}
\hline \multicolumn{7}{|c|}{$\mathrm{m}=2$} \\
\hline $\mathrm{j}$ & 1 & 0 & 1 & 2 & 3 & \multicolumn{1}{c|}{4} \\
\hline 0 & $-2,13 \mathrm{E}-04$ & $-8,894 \mathrm{E}-04$ & $-1,053 \mathrm{E}-02$ & $-3,576 \mathrm{E}-04$ & $-7,019 \mathrm{E}-05$ \\
\hline 1 & $-0,23032756$ & $-0,57469835$ & $-0,12812828$ & $-0,19224541$ & $9,327 \mathrm{E}-03$ \\
\hline 2 & $-2,78035717$ & $-0,4793035$ & $-0,17433774$ & $-0,14232588$ & $-0,51442516$ \\
\hline 3 & 11,1857711 & 3,66043687 & $-0,36924362$ & $-0,17542973$ & $-0,1554302$ \\
\hline 4 & $-14,2504755$ & 0,77928852 & 3,56938342 & $-0,29591237$ & $-0,15653762$ \\
\hline
\end{tabular}

(d)

\begin{tabular}{|c|l|l|l|l|c|c|}
\hline \multicolumn{7}{|c|}{$\mathrm{m}=3$} \\
\hline $\mathrm{j}$ & 1 & 0 & 1 & \multicolumn{1}{c|}{1} & 3 & 4 \\
\hline 0 & $-3,293 \mathrm{E}-04$ & $-2,917 \mathrm{E}-04$ & $-7,996 \mathrm{E}-04$ & $-1,058 \mathrm{E}-02$ & $-4,274 \mathrm{E}-04$ \\
\hline 1 & 0,11463373 & $3,72 \mathrm{E}-02$ & $-0,43116326$ & $-0,12647393$ & $-0,21458735$ \\
\hline 2 & $-2,17106227$ & $-3,0349065$ & $-0,31410728$ & $-0,15871951$ & $-0,15299625$ \\
\hline 3 & $-6,20936757$ & $-1,03819329$ & $-0,35968695$ & $-0,26121832$ & $-0,16386243$ \\
\hline 4 & 25,2347674 & 10,077711 & $-0,7241992$ & $-0,31609004$ & $-0,25773954$ \\
\hline
\end{tabular}

(e)

\begin{tabular}{|c|l|l|l|l|l|c|}
\hline \multicolumn{7}{|c|}{$\mathrm{m}=4$} \\
\hline$j$ & 1 & 0 & \multicolumn{1}{|c|}{1} & \multicolumn{1}{c|}{2} & \multicolumn{1}{c|}{3} & 4 \\
\hline 0 & $-1,718 \mathrm{E}-04$ & $-3,443 \mathrm{E}-04$ & $-2,773 \mathrm{E}-04$ & $-7,583 \mathrm{E}-04$ & $-1,06 \mathrm{E}-02$ \\
\hline 1 & $-3,334 \mathrm{E}-02$ & $1,949 \mathrm{E}-02$ & $3,636 \mathrm{E}-02$ & $-0,38077906$ & $-0,12516759$ \\
\hline 2 & 1,00101369 & 0,10078031 & $-2,04555368$ & $-0,27432121$ & $-0,15398356$ \\
\hline 3 & $-7,85844894$ & $-8,74311655$ & $-0,56707156$ & $-0,28302386$ & $-0,23782895$ \\
\hline 4 & $-11,0041819$ & $-1,8131323$ & $-0,61178354$ & $-0,42524554$ & $-0,25549274$ \\
\hline
\end{tabular}

Uma vez que as autofunções da equação da estrutura vertical do problema de $\mathrm{O}(1)$ obtidas neste trabalho para $\bar{\sigma}$ constante são dadas por uma combinação de seno e co-seno, espera-se que, a priori, a condição de ressonância imposta pelos coeficientes $\alpha_{\mathrm{a}}^{\mathrm{bc}}, \theta_{a}^{b c} \mathrm{e}$ $\vartheta_{a}^{b c}$ seja algo do tipo $\mathrm{m}= \pm \mathrm{j} \pm \mathrm{l}$, ou similarmente, $\lambda_{\mathrm{m}} \approx \pm \lambda_{\mathrm{j}} \pm \lambda_{\mathrm{l}}$. De fato, a partir da Tabela 4.1 é possível observar que os modos verticais $m, j$ e 1 que satisfazem à relação $m= \pm j \pm 1$ apresentam os valores mais significativos dos coeficientes $\alpha_{\mathrm{m}}{ }^{\mathrm{j} 1}$. Conseqüentemente, no caso das interações envolvendo o modo externo, somente as tríades contendo três ondas externas ou tríades compostas por dois modos internos iguais e o modo externo apresentam um acoplamento significativo. Assim, somente os modos verticais satisfazendo à condição $m= \pm$ $\mathrm{j} \pm 1$ interagem significativamente entre si por meio do termo de advecção horizontal de 
momento horizontal. Todavia, os coeficientes de acoplamento $\alpha_{\mathrm{m}}{ }^{\mathrm{j} 1}$ para os modos verticais que não satisfazem esta condição de ressonância, apesar de serem pequenos, não são nulos. Isto se deve ao fato de que os autovalores $\lambda_{m}$ da equação da estrutura vertical não são proporcionais uns aos outros, como é o caso dos números de onda zonal. Logo, a condição de ressonância $m= \pm j \pm 1$ imposta pelos coeficientes $\alpha_{m}{ }^{j 1}$ é seletiva porém não exclusiva, como é o caso das condições de ressonância impostas pelas autofunções em x e em y da solução de ordem dominante.

No caso do acoplamento entre os modos verticais resultante da advecção vertical de momento horizontal, medido predominantemente pelo coeficiente $\theta_{m}^{j l}$, a Tabela 4.2 mostra que a maior parte da projeção no modo externo resulta das interações envolvendo o próprio modo externo e um modo interno, especialmente o primeiro modo baroclínico $1=1$. É interessante observar que as tríades contendo $1=0$ apresentam os coeficientes de interação $\theta_{m}^{j l}$ pequenos, devido à pequena variação desse modo vertical com a pressão. Assim como no caso dos coeficientes de acoplamento resultantes da advecção horizontal de momento horizontal, as auto-interações entre os modos internos também projetam significativamente no modo barotrópico $\mathrm{m}=0$, como mostrado na Tabela 4.2. Com relação às tríades com $\mathrm{m}>0$, é interessante notar a partir da Tabela 4.2 que os acoplamentos mais significativos estão associados aos tripletos contendo o modo barotrópico $\mathrm{j}=0$ e um outro modo baroclínico, especialmente o modo interno $1>0$ que satisfaz à relação $1=\mathrm{m}+1$. Para as tríades contendo somente modos internos, observa-se a partir da Tabela 4.2 que somente os modos satisfazendo à condição $m= \pm j \pm 1$ interagem expressivamente entre si por meio do termo de advecção vertical de momento horizontal.

No caso do acoplamento entre os modos verticais resultante da advecção horizontal diferencial de espessura com a pressão (ou advecção horizontal térmica diferencial com a altura), medido pelos coeficientes $\vartheta_{m}^{j l}$, a Tabela 4.3 mostra que a projeção no modo externo por meio dessas interações é pequena. Por outro lado, as projeções significativas num modo interno qualquer resultantes desse termo não linear estão sempre associadas às interações triádicas que envolvem modos verticais que satisfazem à relação $\mathrm{m}= \pm \mathrm{j} \pm 1$. Conseqüentemente, no caso de uma tríade contendo um modo com estrutura barotrópica e dois modos com estrutura baroclínica, as interações mais intensas referem-se sempre às tríades nas quais os dois modos internos são iguais. 
Para os coeficientes $\varsigma_{m}^{j l}$, como pode ser observado a partir da equação (2.94), alguns dos termos envolvidos no cálculo desses coeficientes possuem produtos triplos contendo "funções peso" do tipo $1 / \mathrm{p}$ ou $1 / \mathrm{p}^{2}$, que dão uma ponderação maior para os valores das funções na alta troposfera. Conseqüentemente, a condição de ressonância vertical aproximada dada por $m= \pm \mathrm{j} \pm 1$, verificada nas interações associadas aos coeficientes $\alpha_{\mathrm{a}}^{\mathrm{bc}}, \theta_{a}^{b c} \mathrm{e} \vartheta_{a}^{b c}$, é bem menos restritiva para as interações verticais medidas pelos coeficientes $\varsigma_{m}^{j l}$, como pode ser observado na Tabela 4.4. De fato, a partir da Tabela 4.4 pode ser observado que as interações triádicas envolvendo somente modos baroclínicos são, em geral, mais expressivas quando os modos satisfazem à relação $m= \pm j \pm 1$. Porém, é possível notar que as interações correspondentes aos tripletos que não satisfazem esta condição são bem menos inexpressivas que as mesmas interações no caso dos coeficientes $\alpha_{a}^{b c}, \theta_{a}^{b c}$ e $\vartheta_{a}^{b c}$. Como resultado, o espectro de ressonância decorrente das interações verticais medidas pelos coeficientes $\varsigma_{m}^{j l}$ dispostos na Tabela 4.4 é bem mais amplo em comparação com os espectros de ressonância associados aos coeficientes $\alpha_{\mathrm{a}}^{\mathrm{bc}}, \theta_{a}^{b c}$ e $\vartheta_{a}^{b c}$, permitindo um número bem maior de interações. As interações medidas pelos coeficientes $\varsigma_{m}^{j l}$ mais significativas são aquelas associadas aos tripletos constituídos por $\mathrm{j}=0$ e modos baroclínicos com 1 maiores, como mostrado na Tabela 4.4.

Os coeficientes de interação entre os modos verticais devidos aos termos não lineares na fronteira vertical do domínio, dados por $-\left.\left(\frac{\widetilde{\bar{\rho}}}{c_{b}{ }^{2}}+\widetilde{\bar{\Gamma}}\right) \frac{G_{a}(p) G_{b}(p) G_{c}(p)}{\left\|G_{a}\right\|^{2}}\right|_{1} ^{\tilde{p}_{T}} \mathrm{e}$ $\left.\Lambda \widetilde{\bar{\rho}} \frac{G_{a}(p) G_{b}(p) G_{c}(p)}{\left\|G_{a}\right\|^{2}}\right|_{1} ^{\widetilde{p}_{T}}$, apresentam valores menores que $10^{-2} \mathrm{e}$, portanto, são muito pequenos em comparação com os coeficientes $\alpha_{\mathrm{a}}^{\mathrm{bc}}, \theta_{a}^{b c}, \vartheta_{a}^{b c}$ e $\varsigma_{a}^{b c}$. Logo, estes coeficientes, assim como os coeficientes $\mu_{\mathrm{a}}^{\mathrm{bc}} \mathrm{e} \Psi_{a}^{b c}$, não são levados em consideração na determinação dos modos verticais mais ressonantes. Entretanto, apesar desses coeficientes não tiverem sido levados em consideração na escolha dos tripletos ressonantes, é importante ressaltar que eles não foram desprezados nos cálculos dos coeficientes de interação $\eta_{\mathrm{a}}{ }^{\mathrm{bc}}$ para as tríades ressonantes determinadas nesse capítulo. Inclusive, como será mostrado mais adiante, o termo não linear nas fronteiras verticais que aparece no cálculo dos coeficientes de acoplamento $\eta_{\mathrm{a}}{ }^{\mathrm{bc}}$ 
em (2.92) é importante para a dinâmica dos tripletos ressonantes que envolvem ondas de gravidade-inerciais.

Dessa forma, de um modo geral, pode ser notado a partir das Tabelas 4.1 a 4.4 que os modos verticais que satisfazem à condição $m= \pm j \pm 1$ apresentam as interações mais significativas. Inclusive, analisando as Tabelas 4.1 a 4.4 pode ser notado que os coeficientes de interação $\alpha_{\mathrm{a}}^{\mathrm{bc}}, \theta_{a}^{b c}, \vartheta_{a}^{b c}$ e $\varsigma_{a}^{b c}$ são pequenos para as tríades ressonantes nas quais as três ondas apresentam o mesmo modo interno. Isto implica que a contribuição de ordem dominante dos termos não lineares torna-se pequena ao truncar o modelo aqui considerado de forma a considerar somente um único modo interno na representação da estrutura vertical. Com isso, embora a condição $m= \pm j \pm 1$ para os modos verticais não seja exclusiva e sim seletiva e também existam outras tríades de modos verticais que não satisfazem tal condição e possam apresentar interações relevantes, neste trabalho será dado enfoque somente às interações triádicas cujos modos verticais associados às ondas constituintes das tríades ressonantes satisfazem à condição $\mathrm{m}= \pm \mathrm{j} \pm 1$. Além disso, dado que o objetivo do presente estudo consiste em analisar o papel da não linearidade na interação entre as circulações atmosféricas tropical e extra-tropical, ou seja, explorar a interação entre modos de variabilidade atmosféricos tropicais e extra-tropicais, somente os tripletos envolvendo dois modos com a mesma estrutura baroclínica e uma onda de Rossby externa serão analisados. As ondas de Rossby externas, como discutido no Capítulo 2, estão associadas aos padrões globais de teleconexões existentes na atmosfera. Estes padrões de teleconexões ocorrem tipicamente na escala de baixa freqüência, predominantemente na escala intra-sazonal e inter-anual, e envolvem tanto regiões tropicais quanto extra-tropicais (Grimm e Silva Dias 1995). A dinâmica de tríades ressonantes envolvendo somente ondas de Rossby barotrópicas foi explorada por Longuet-Higgins e Gill (1967), utilizando um modelo de um fluido barotrópico e não divergente. Além da energia cinética total, estas interações conservam a enstrofia total. Como resultado, nas interações não lineares triádicas das ondas de Rossby barotrópicas não divergentes, a onda que apresenta o número de onda intermediário é sempre a componente instável do tripleto, ou seja, sempre recebe energia das outras duas ou cede energia para as demais componentes do tripleto. Como os coeficientes de interação $\theta_{a}^{b c}, \vartheta_{a}^{b c}$ e $\varsigma_{a}^{b c}$ para as tríades constituídas somente por ondas barotrópicas são pequenos e as ondas de Rossby barotrópicas obtidas como uma das componentes da solução de ordem dominante do modelo atmosférico aqui considerado possuem uma divergência pequena, os tripletos envolvendo somente ondas de Rossby externas no modelo aqui adotado devem apresentar 
aproximadamente as mesmas propriedades dos tripletos estudados por Longuet-Higgins e Gill. No caso dos demais tripletos, como mostrado no Capítulo 3, a única propriedade garantida é que a onda com a maior freqüência absoluta representa a componente que cede energia para as outras duas ondas ou recebe energia das mesmas.

Dessa forma, no presente capítulo desta tese serão determinados alguns tripletos de ondas satisfazendo às condições de ressonância dadas por (4.1) e que são constituídos por uma onda de Rossby barotrópica e duas ondas quaisquer que apresentem a mesma estrutura baroclínica na vertical, ou seja, correspondentes ao mesmo modo interno. Para tal propósito, utilizou-se um método gráfico no qual uma tríade é determinada através da intersecção de duas curvas de dispersão quaisquer onde uma das curvas é simetricamente deslocada no plano cartesiano $\varpi(k)$ Vs k ao longo de uma outra curva de dispersão, de tal maneira que a origem de uma das curvas intersectadas coincida com o número de onda e a freqüência temporal de um outro modo em particular, caracterizado por um modo vertical $\mathrm{m}$, um número de onda zonal $\mathrm{k}$ e por um modo meridional $\mathrm{n}$. É importante ressaltar que, uma vez que o domínio zonal do modelo atmosférico aqui considerado é periódico, a ressonância exata para as freqüências temporais somente é obtida se a intersecção das duas curvas de dispersão ocorrer exatamente em um dos números de onda definidos, dados por $\mathrm{k}=\frac{2 \pi}{\widetilde{L}_{x}} j, \mathrm{j}=0, \pm 1, \pm 2, \ldots$. Apesar dessa intersecção exata ser muito difícil de ocorrer na prática, se ela ocorrer próxima a algum ponto na curva de dispersão onde o número de onda zonal é definido, a pressão aproximada da "parede superior" do modelo, dada por $\widetilde{p}_{T}$, pode ser ajustada de tal maneira que a intersecção ocorra o mais próxima possível do ponto onde o número de onda zonal é definido e, portanto, de modo que a ressonância obtida esteja o mais próxima possível da ressonância exata, ou seja, de tal maneira que a tolerância $\Delta$ na condição de ressonância (4.1a) seja dada por $\Delta=\mathrm{O}(\varepsilon)$.

A Figura 4.1 mostra a determinação de uma tríade ressonante através desse método gráfico para $\bar{\sigma}=2 \times 10^{-6} \mathrm{~m}^{4} \mathrm{~s}^{2} \mathrm{Kg}^{-2}, \mathrm{p}_{0}=1000 \mathrm{hPa}$ e $\mathrm{p}_{\mathrm{T}}=200 \mathrm{hPa}$. A tríade ressonante determinada na Figura 4.1 é constituída por uma onda de Rossby barotrópica com número de onda zonal 4 , que corresponde a $\mathrm{k} \approx 0,628$, e modo meridional $\mathrm{n}=2$, uma onda mista de Rossby-gravidade com o mesmo número de onda zonal e com a estrutura vertical correspondente ao primeiro modo baroclínico $\mathrm{m}=1$ e um modo de Rossby baroclínico $(\mathrm{m}=$ 1) zonalmente simétrico $(\mathrm{k}=0)$ cujo modo meridional é dado por $\mathrm{n}=1$. Os modos de Rossby zonalmente simétricos apresentam a componente meridional do vento identicamente nula e 
são caracterizados por um balanço geostrófico exato $\left(\varpi_{\mathrm{a}}=0\right)$ entre a componente zonal do vento e o gradiente meridional do geopotencial (Silva Dias e Schubert 1979; Raupp 2002), como discutido no Capítulo 2. Nesta figura, assim como nas Figuras 4.2 a 4.4, as curvas de dispersão com pontos marcados ao longo das mesmas referem-se às ondas equatoriais internas, enquanto a curva contínua refere-se às ondas de Rossby barotrópicas. As curvas com os números de onda zonal $\mathrm{k}$ negativos correspondem às ondas se propagando para leste, enquanto as curvas com k positivo referem-se às ondas com propagação de fase para oeste. Os símbolos marcados ao longo das curvas de dispersão destacam os pontos onde os números de onda zonal são definidos. É notável na Figura 4.1 a ressonância quase exata da onda de Yanai associada ao primeiro modo baroclínico com número de onda zonal $4(\mathrm{k} \approx 0,628)$ e a onda de Rossby barotrópica com o mesmo número de onda e com o modo meridional $\mathrm{n}=2$.

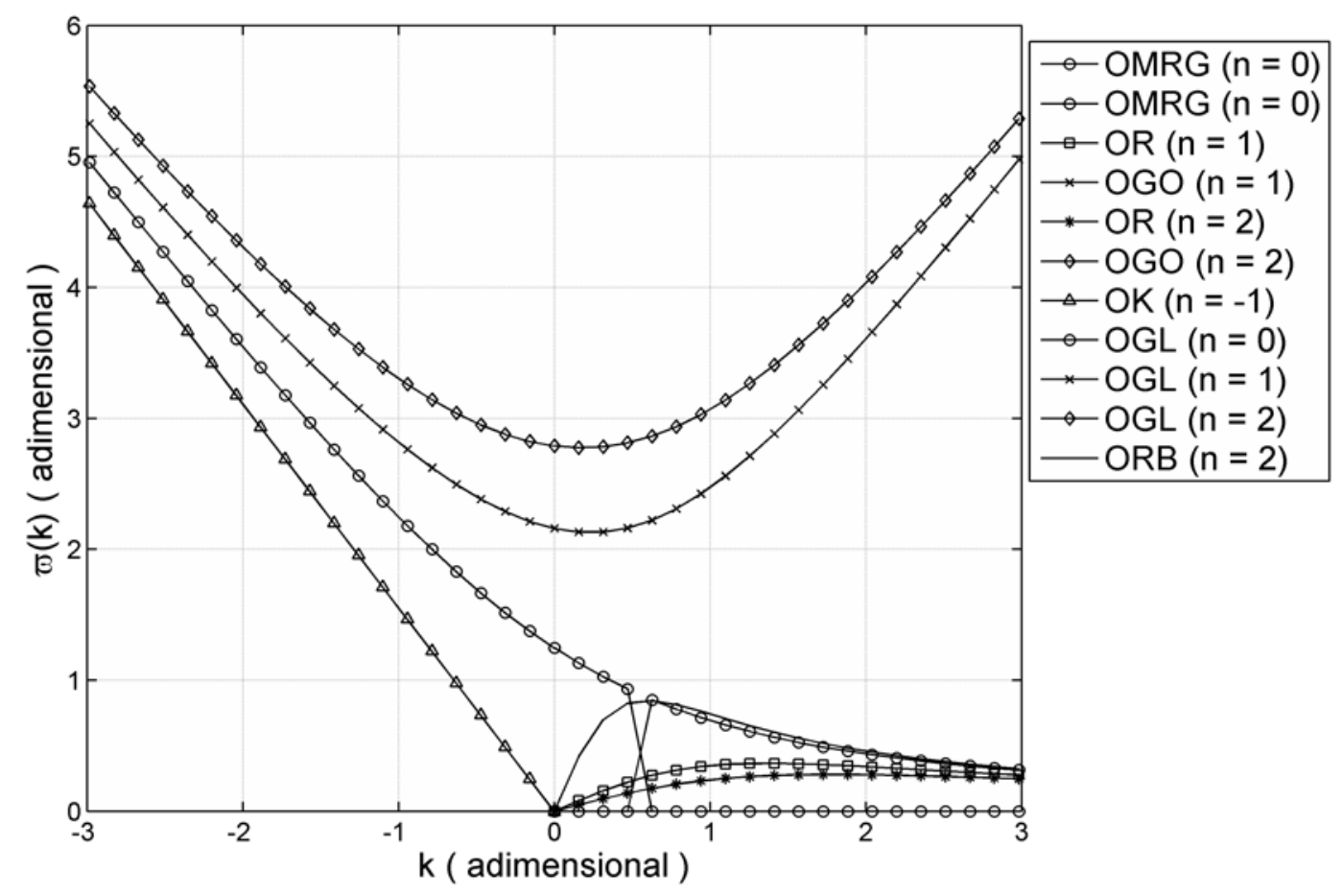

Figura 4.1: Diagrama de dispersão das ondas de Rossby barotrópicas (ORB) com o modo meridional $\mathrm{n}=2$ e das seguintes ondas equatoriais associadas ao primeiro modo baroclínico $\mathrm{m}$ = 1: ondas mistas de Rossby-gravidade (OMRG), ondas de Rossby (OR) para os modos meridionais $n=1$ e $n=2$, ondas de gravidade-inerciais com propagação para leste associadas ao modo meridional $\mathrm{n}=0$, ondas de gravidade-inerciais para oeste (OGO) e para leste (OGL) para os modos meridionais $n=1$ e $n=2$ e das ondas de Kelvin (OK). As curvas destacam a 
ressonância envolvendo as ondas de Rossby barotrópicas e as ondas mistas de Rossbygravidade por meio do modo geostrófico zonalmente simétrico com modo meridional $\mathrm{n}=1$.

Além dessa ressonância, também é interessante observar na Figura 4.1 que todas as componentes da onda de Yanai associada ao primeiro modo baroclínico $\mathrm{m}=1$ que estejam inseridas no regime de baixa freqüência (i.e., com $\mathrm{k}>2^{-1 / 2}$ ) são ressonantemente acopladas com as ondas de Rossby barotrópicas com $n=2$ e os mesmos números de onda zonal por meio do modo geostrófico zonalmente simétrico com $n=1$ e associado ao primeiro modo baroclínico, para os valores de $\bar{\sigma}, \mathrm{p}_{0}$ e $\mathrm{p}_{\mathrm{T}}$ aqui considerados. É importante destacar que a condição de ressonância em y dada por (4.1c) é satisfeita para todos esses tripletos. Outro aspecto importante a ser mencionado é que, apesar das ressonâncias para os números de onda 5 a 11 serem menos exatas que a ressonância referente ao número de onda 4 , o resíduo da equação (4.1a) para estes tripletos é apenas da ordem de $10^{-2}$. A ressonância verificada na Figura 4.1 envolve um conjunto de tripletos ressonantes envolvendo ondas de Rossby barotrópicas e ondas de Yanai internas, com o mesmo número de onda zonal $\mathrm{k}>2^{-1 / 2}$, acoplados por meio de um único modo, o modo geostrófico zonalmente simétrico associado ao primeiro modo baroclínico $m=1$ e com o modo meridional $n=1$. Esta ressonância obtida na Figura 4.1 parece ser bastante robusta e, conseqüentemente, constituir um mecanismo importante na conexão trópicos-extratrópicos. A dinâmica desses tripletos ressonantes, bem como as implicações dessa dinâmica para a questão da interação entre trópicos e extratrópicos, será abordada no Capítulo 5.

A partir da Figura 4.1 também é possível notar que as ondas de Rossby, tanto barotrópicas quanto baroclínicas, tendem a ser não dispersivas no limite assintótico de ondas ultra-longas $(\mathrm{k} \rightarrow 0)$. Logo, as ondas de Rossby barotrópicas também são ressonantemente acopladas às ondas de Rossby baroclínicas no limite do espectro referente às ondas ultralongas. Majda e Biello (2003) exploraram a dinâmica dessa ressonância usando as equações de Boussinesq para um modelo troposférico de duas camadas, como discutido no Capítulo 1. É importante ressaltar que esta ressonância estudada por Majda e Biello não é triádica, uma vez que as ondas de Rossby no limite $\mathrm{k} \rightarrow 0$ são não dispersivas. De fato, a dinâmica reduzida para essas interações ressonantes é governada por um sistema de duas equações diferenciais parciais acopladas, uma descrevendo a evolução do "pacote" de ondas de Rossby equatoriais baroclínicas e a outra descrevendo a evolução do "pacote" de ondas de Rossby barotrópicas. No caso da ressonância determinada na Figura 4.1 envolvendo ondas de Rossby barotrópicas 
e ondas de Yanai com o modo baroclínico $\mathrm{m}=1$, como essas ondas ressonantes são dispersivas, não há um auto-acoplamento entre as próprias componentes do pacote de ondas de Yanai e de Rossby. Dois dos tripletos ressonantes envolvendo as ondas de Rossby barotrópicas e as ondas de Yanai internas encontrados através da Figura 4.1 são mostrados na Tabela 4.5.

A Figura 4.2 ilustra a determinação de uma tríade ressonante contendo uma onda de Rossby barotrópica com $\mathrm{n}=1$ e número de onda $7(\mathrm{k} \approx 1,1)$, e duas ondas de Poincaré propagando para oeste com modo vertical $\mathrm{m}=1$ e modo meridional $\mathrm{n}=1$, sendo uma apresentando número de onda zonal $1(\mathrm{k} \approx 0,157)$ e a outra número de onda zonal $8(\mathrm{k} \approx$ 1,256). Na determinação dessa tríade ressonante mostrada na Figura 4.2 foram utilizados $\bar{\sigma}=$ $2 \times 10^{-6} \mathrm{~m}^{4} \mathrm{~s}^{2} \mathrm{Kg}^{-2}, \mathrm{p}_{0}=1000 \mathrm{hPa}$ e $\mathrm{p}_{\mathrm{T}}=80 \mathrm{hPa}$. É interessante notar que uma outra tríade ressonante também é encontrada na Figura 4.2. Esta tríade contém uma onda de Rossby barotrópica com número de onda 1 e $n=1$ e duas ondas de Poincaré baroclínicas com propagação para oeste, uma com número de onda 1 e $n=1$ e a outra com número de onda 2 e $\mathrm{n}=2$. Porém, os modos constituintes dessa tríade não satisfazem à condição $(4.1 \mathrm{c}) \mathrm{e}$, portanto, os coeficientes de interação referentes a esta tríade são nulos (ver Tabela 4.5). $\mathrm{O}$ mesmo ocorre com as tríades ressonantes envolvendo ondas de Rossby externas com modo meridional $\mathrm{n}=1$ e ondas de Yanai $(\mathrm{n}=0)$ e de Poincaré com modo meridional $\mathrm{n}=1$, ambas associadas ao primeiro modo baroclínico $\mathrm{m}=1$ (figura não mostrada).

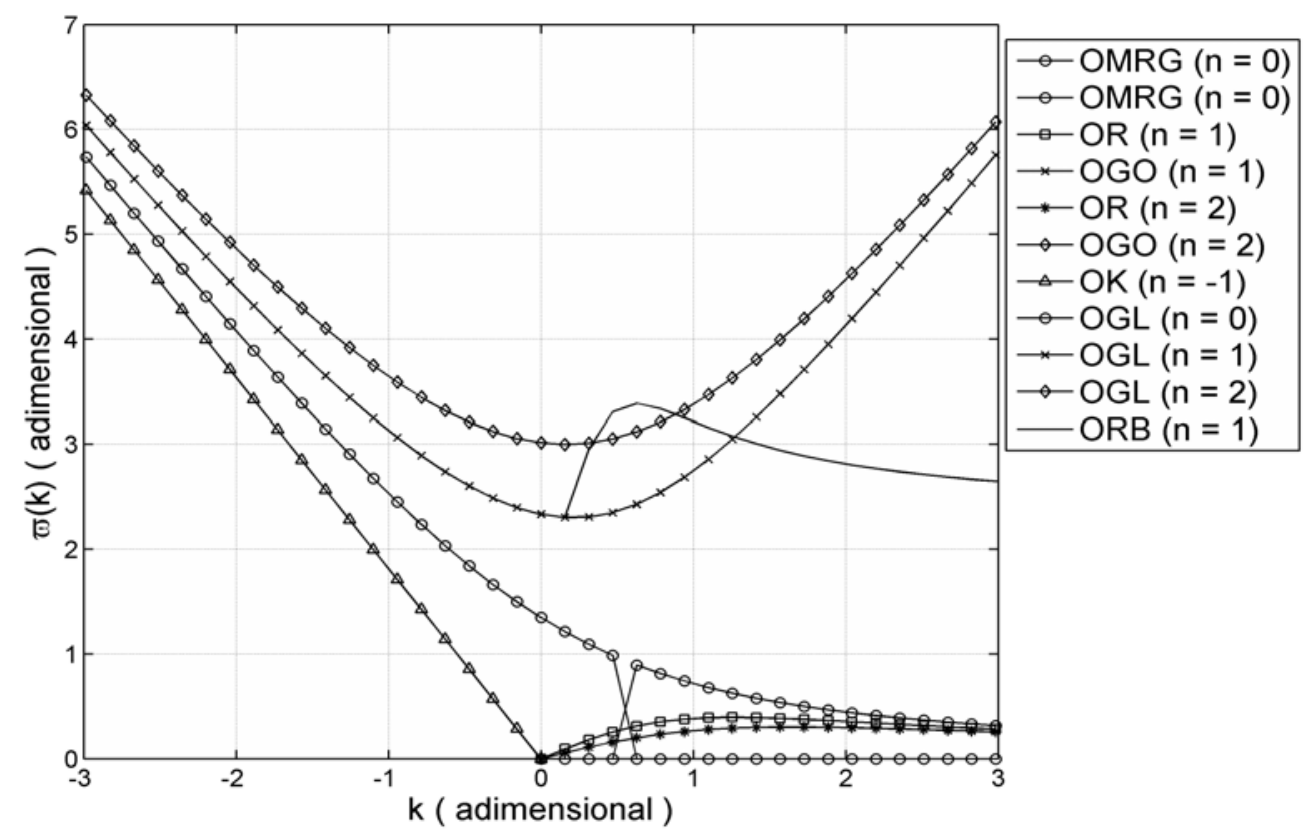

Figura 4.2: Diagrama de dispersão das ondas de Rossby barotrópicas (ORB) com o modo meridional $\mathrm{n}=1$ e das seguintes ondas equatoriais para o primeiro modo baroclínico $\mathrm{m}=1$ : 
ondas mistas de Rossby-gravidade (OMRG), ondas de Rossby (OR) para os modos meridionais $n=1$ e $n=2$, ondas de gravidade-inerciais com propagação para leste associadas ao modo meridional $\mathrm{n}=0$, ondas de gravidade-inerciais para oeste (OGO) e para leste (OGL) com modos meridionais $\mathrm{n}=1$ e $\mathrm{n}=2$ e das ondas de Kelvin (OK). As curvas destacam a ressonância envolvendo a onda de Rossby barotrópica com $\mathrm{n}=1$ e número de onda $7(\mathrm{k} \approx 1,1)$ e duas ondas de Poincaré baroclínicas (modo vertical $\mathrm{m}=1$ ) e com modo meridional $\mathrm{n}=1$, sendo uma com número de onda $1(\mathrm{k} \approx 0,157)$ e a outra com número de onda $8(\mathrm{k} \approx 1,256)$.

Como a curva de dispersão das ondas de Rossby externas para $\mathrm{n}=2$ não intercepta as curvas de dispersão das ondas de gravidade-inerciais associadas ao primeiro modo baroclínico, mesmo com a origem coincidindo sobre um dos pontos ao longo da curva de dispersão das ondas mistas de Rossby-gravidade, tríades ressonantes envolvendo ondas de Rossby externas e ondas internas de Yanai e de gravidade-inerciais somente são possíveis para o caso de um modo interno mais alto, como ilustrado na Figura 4.3. A Figura 4.3 mostra a existência de uma tríade ressonante composta por uma onda de Rossby barotrópica com número de onda $4(\mathrm{k} \approx 0,628)$ e $\mathrm{n}=2$ e duas ondas equatoriais associadas ao modo vertical $\mathrm{m}$ $=2$, sendo elas: uma onda mista de Rossby-gravidade com número de onda $1(\mathrm{k} \approx 0,157) \mathrm{e}$ uma onda de gravidade-inercial com propagação para oeste com número de onda 5 e $n=1$. A determinação dessa tríade se deu para os mesmos valores de $\bar{\sigma}, \mathrm{p}_{0}$ e $\mathrm{p}_{\mathrm{T}}$ usados na Figura 4.2. 


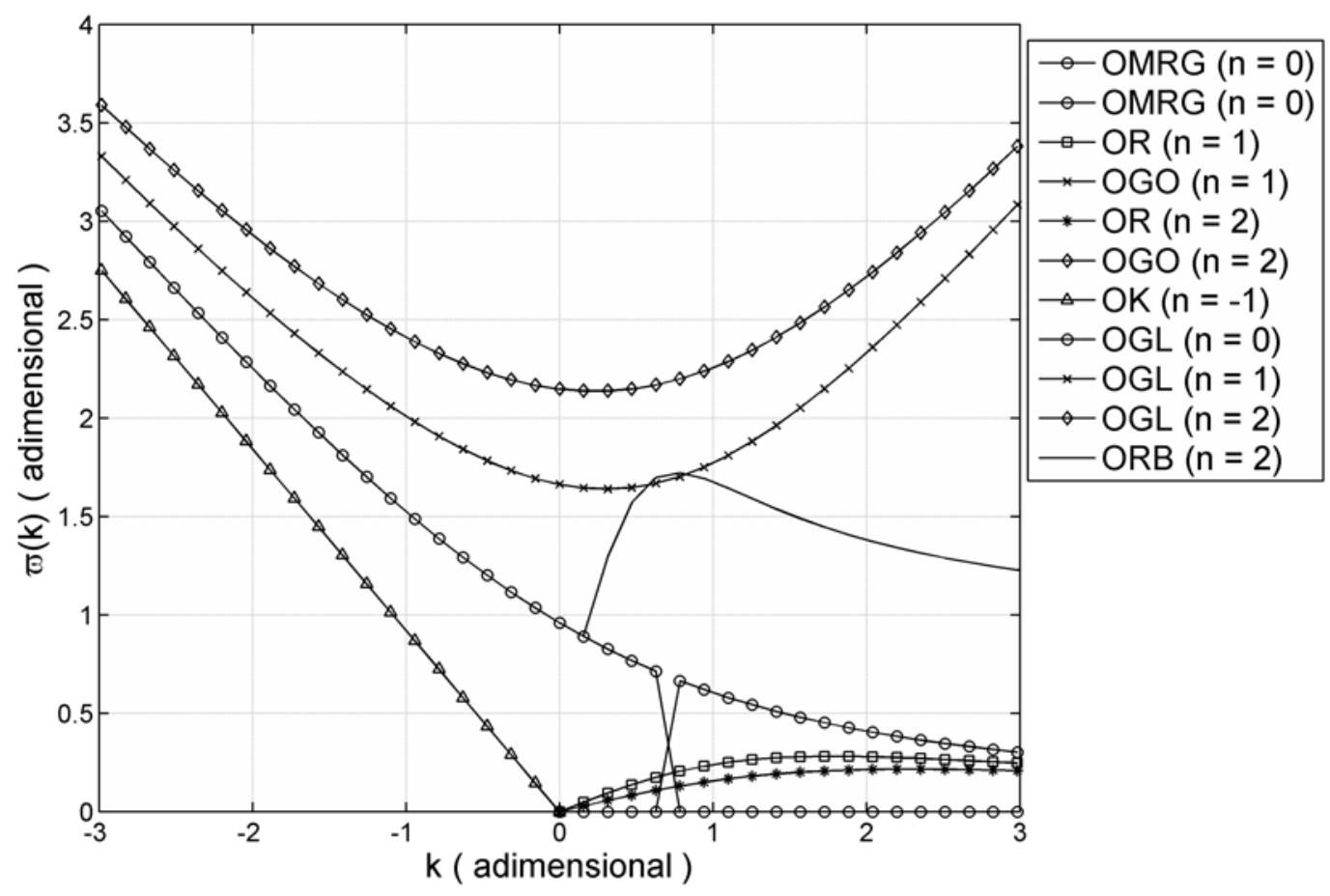

Figura 4.3: Diagrama de dispersão das ondas de Rossby barotrópicas (ORB) com o modo meridional $\mathrm{n}=2$ e das seguintes ondas equatoriais para o segundo modo baroclínico $(\mathrm{m}=2)$ : ondas mistas de Rossby-gravidade (OMRG), ondas de Rossby (OR) para os modos meridionais $n=1$ e $n=2$, ondas de gravidade-inerciais com propagação para leste (OGL) associadas ao modo meridional $\mathrm{n}=0$, ondas de gravidade-inerciais para oeste (OGO) e para leste (OGL) com modos meridionais $n=1$ e $n=2$ e das ondas de Kelvin (OK). As curvas destacam a ressonância envolvendo a onda de Rossby barotrópica com $n=2$ e número de onda $4(\mathrm{k} \approx 0,628)$ e duas ondas equatoriais baroclínicas (modo vertical $\mathrm{m}=2)$, sendo elas: uma onda mista de Rossby-gravidade com número de onda $1(\mathrm{k} \approx 0,157)$ e uma onda de gravidade-inercial para oeste com número de onda 5 e $n=1$.

A Figura 4.4 mostra a existência de uma tríade ressonante envolvendo uma onda de Kelvin com número de onda zonal $1(\mathrm{k} \approx 0,157)$, uma onda de Yanai com número de onda 2 $(\mathrm{k} \approx 0,314)$, ambas com a estrutura vertical referente ao modo baroclínico $\mathrm{m}=1$, e uma onda de Rossby barotrópica com modo meridional $\mathrm{n}=2$ e número de onda zonal $3(\mathrm{k} \approx 0,471)$. Esta tríade foi obtida utilizando os parâmetros $\bar{\sigma}=2 \times 10^{-6} \mathrm{~m}^{4} \mathrm{~s}^{2} \mathrm{Kg}^{-2}, \mathrm{p}_{0}=1000 \mathrm{hPa}$ e $\mathrm{p}_{\mathrm{T}}=$ $100 \mathrm{hPa}$. Este tripleto ressonante receberá uma atenção especial no próximo capítulo desta tese 
devido à sua possível relação com diversos fatores observados acerca da variabilidade de baixa freqüência da circulação atmosférica de grande-escala.

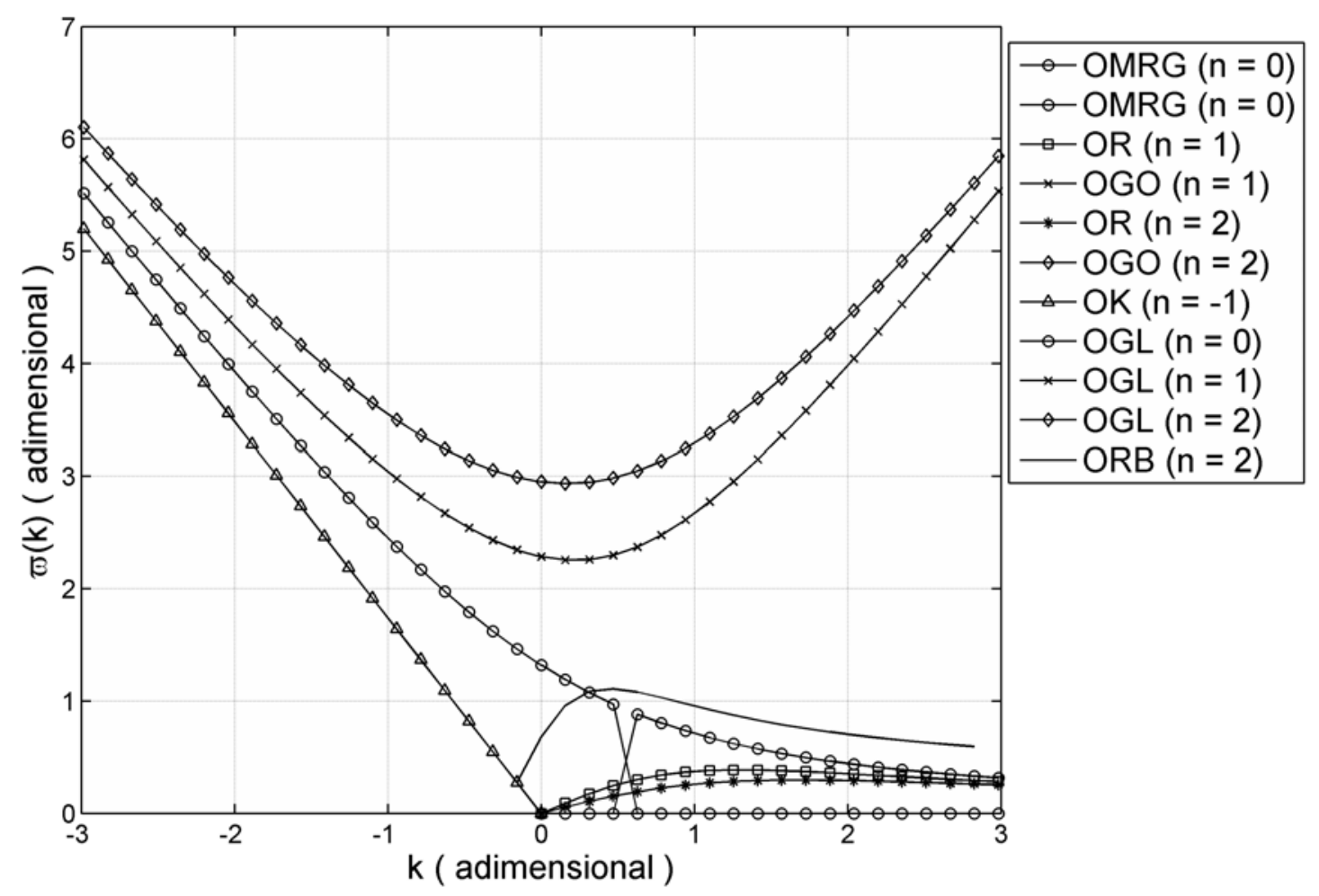

Figura 4.4: Diagrama de dispersão das ondas de Rossby barotrópicas (ORB) com o modo meridional $\mathrm{n}=2$ e das seguintes ondas equatoriais correspondentes ao primeiro modo baroclínico $\mathrm{m}=1$ : ondas mistas de Rossby-gravidade (OMRG), ondas de Rossby (OR) para os modos meridionais $n=1$ e $n=2$, ondas de gravidade-inerciais com propagação para leste associadas ao modo meridional $\mathrm{n}=0$, ondas de gravidade-inerciais para oeste $(\mathrm{OGO})$ e para leste (OGL) com os modos meridionais $n=1$ e $n=2$ e das ondas de Kelvin (OK). As curvas destacam a ressonância envolvendo a onda de Rossby barotrópica com $n=2$ e número de onda $3(\mathrm{k} \approx 0,471)$ e duas ondas equatoriais baroclínicas (modo vertical $\mathrm{m}=1)$, sendo estas: uma onda de Kelvin com número de onda zonal $1(\mathrm{k} \approx 0,157)$ e uma onda mista de Rossbygravidade com número de onda zonal $2(\mathrm{k} \approx 0,314)$. 
Um sumário das tríades ressonantes determinadas neste capítulo é apresentado na Tabela 4.5. Além dos modos constituintes das tríades ressonantes, a Tabela 4.5 mostra as respectivas autofreqüências e os coeficientes de interação a elas associados. Os coeficientes de interação $\eta_{\mathrm{a}}^{\mathrm{bc}}$ foram calculados numericamente a partir de (2.92) através do método da Quadratura de Gauss-Hermite. As derivadas das autofunções $\xi_{\mathrm{a}}(\mathrm{y})$ envolvidas nesse cálculo foram obtidas analiticamente a partir das equações (2.45), (2.50) e (2.51). As fórmulas para essas derivadas são mostradas no Apêndice C. É importante observar na Tabela 4.5 que, de fato, a soma dos coeficientes de interação $\eta_{\mathrm{a}}{ }^{\mathrm{bc}}, \eta_{\mathrm{b}}{ }^{\mathrm{ac}}$, e $\eta_{\mathrm{c}}{ }^{\mathrm{ab}}$ é aproximadamente nula para todos os tripletos de ondas ressonantes que interagem determinados neste capítulo. O resíduo na soma dos coeficientes de interação das tríades dispostas na Tabela 4.5 pode ser atribuído ao fato de as ressonâncias obtidas não serem exatas para as freqüências temporais, bem como ao erro de truncamento nos métodos de integração numérica envolvidos no cálculo desses coeficientes. Porém, pode ser verificado que esse resíduo é da ordem de $10^{-2}$ para todas as tríades mostradas na Tabela 4.5, sendo, na maioria dos tripletos, menor que o valor absoluto do menor coeficiente.

Tabela 4.5: Sumário das tríades ressonantes obtidas pelo método gráfico mostradas nas Figuras 4.1 a 4.4. A Tabela mostra, da esquerda para a direita, os modos constituintes das tríades e suas respectivas auto-freqüências e coeficientes de acoplamento. Os modos são caracterizados, da esquerda para a direita, pelo modo vertical, pelo número de onda zonal, pelo modo meridional e pelo tipo de onda. Os tipos de ondas mostrados são abreviados como segue: Rossby (R), mista Rossby-gravidade (M), Kelvin (K), gravidade-inerciais com propagação para oeste $(\mathrm{GO})$ e ondas de gravidade-inerciais com propagação para leste (GL).

\begin{tabular}{|l|l|l|l|l|l|l|l|l|l|}
\hline & $\mathrm{a}$ & $\mathrm{b}$ & $\mathrm{c}$ & $\mathrm{w}_{\mathrm{a}}$ & $\varpi_{\mathrm{b}}$ & $\varpi_{\mathrm{c}}$ & $\mathrm{i} \eta_{\mathrm{a}}{ }^{\mathrm{bc}}$ & $\mathrm{i} \eta_{\mathrm{b}}{ }^{\mathrm{ac}}$ & $\mathrm{i} \eta_{\mathrm{c}}{ }^{\mathrm{ab}}$ \\
\hline 1 & $0,4,2, \mathrm{R}$ & $1,4,0, \mathrm{M}$ & $1,0,1, \mathrm{R}$ & 0,8438 & 0,851 & 0 & $-0,455$ & 0,4335 & 0,00 \\
\hline 2 & $0,6,2, \mathrm{R}$ & $1,6,0, \mathrm{M}$ & $1,0,1, \mathrm{R}$ & 0,761 & 0,714 & 0 & 3,0368 & $-2,985$ & 0,00 \\
\hline 3 & $1,2,2, \mathrm{GO}$ & $1,1,1, \mathrm{GO}$ & $0,1,1, \mathrm{R}$ & 3,0112 & 2,3039 & 0,6489 & 0 & 0 & 0 \\
\hline 4 & $1,8,1, \mathrm{GO}$ & $1,1,1, \mathrm{GO}$ & $0,7,1, \mathrm{R}$ & 3,0497 & 2,3039 & 0,7717 & $-0,329$ & 0,2181 & 0,0345 \\
\hline 5 & $2,5,1, \mathrm{GO}$ & $2,1,0, \mathrm{M}$ & $0,4,2, \mathrm{R}$ & 1,7022 & 0,8905 & 0,83 & $-0,12$ & 0,0685 & 0,0333 \\
\hline 6 & $1,2,0, \mathrm{M}$ & $1,1,-1, \mathrm{~K}$ & $0,3,2, \mathrm{R}$ & 1,0747 & $-0,274$ & 0,8126 & $-1,349$ & 1,1561 & 0,1221 \\
\hline
\end{tabular}


Conseqüentemente, pode ser notado a partir dos valores dispostos na Tabela 4.5 que nas tríades ressonantes aqui determinadas o modo com maior freqüência absoluta apresenta sempre o coeficiente de interação com sinal oposto ao dos outros dois membros e, por conseguinte, com maior valor absoluto. Logo, nas tríades ressonantes dispostas na Tabela 4.5 o modo com a maior freqüência absoluta sempre age como uma fonte ou como um sumidouro de energia para as outras duas componentes. Também é interessante notar na Tabela 4.5 que, em geral, os coeficientes de interação numa tríade ressonante são proporcionais às respectivas freqüências absolutas dos modos a eles associados. A única exceção mostrada na Tabela 4.5 ocorre para a tríade 6 , na qual apesar do resíduo ser muito pequeno tanto na condição de ressonância (4.1a) quanto na soma dos coeficientes de interação para os modos constituintes dessa tríade, o modo de Rossby barotrópico apresenta uma freqüência absoluta maior que a do modo de Kelvin interno mas apresenta o coeficiente de interação bem menor que aqueles associados aos modos de Kelvin e de Yanai. Outro ponto importante a ser observado é que nas tríades ressonantes envolvendo os modos geostróficos zonalmente simétricos, como conseqüência da freqüência nula desses modos, os coeficientes de interação referentes a esses modos são sempre nulos e, por conseguinte, esses modos não trocam energia com as outras duas ondas nos tripletos ressonantes. Os modos absolutamente geostróficos agem somente somo catalisadores, proporcionando a troca de energia dos dois modos restantes nas tríades ressonantes. É notável na Tabela 4.5 o intenso acoplamento entre a onda de Rossby barotrópica com número de onda 6 e $\mathrm{n}=2$ e a onda mista de Rossby-gravidade com o mesmo número de onda e com o modo vertical $\mathrm{m}=1$ por meio do modo geostrófico zonalmente simétrico com modo meridional $\mathrm{n}=1$ e com o mesmo modo baroclínico associado à onda mista. Como resultado do acoplamento mais intenso dos modos constituintes do tripleto 2 na Tabela 4.5, a onda de Rossby externa e a onda de Yanai interna no tripleto 2 trocam energia numa escala de tempo mais curta em comparação com os modos constituintes dos demais tripletos. Por exemplo, nas integrações das equações (2.96) para as tríades 2 e 6, que serão exploradas no Capítulo 5, a onda de Rossby barotrópica e a onda de Yanai baroclínica constituintes da tríade 2 na Tabela 4.5 trocam energia numa escala de tempo mais curta que as ondas constituintes da tríade 6 , como será mostrado no capítulo seguinte. Como também será mostrado no Capítulo 5 para a tríade 2 da Tabela 4.5, embora os modos geostróficos zonalmente simétricos ajam somente como catalisadores nas tríades ressonantes, não trocando energia com os demais modos, a amplitude inicial desse modo numa interação triádica ressonante controla o período da troca de energia das outras duas ondas. 
Um outro aspecto interessante a ser explorado acerca das interações não lineares ressonantes estudadas no presente trabalho refere-se à significância dos termos não lineares nas fronteiras verticais do domínio resultantes do segundo termo nas expansões em série de Taylor em (2.8). A Tabela 4.6 mostra as mesmas tríades ressonantes dispostas na Tabela 4.5, mas com os coeficientes de interação $\eta_{\mathrm{a}}{ }^{\mathrm{bc}}$ calculados considerando artificialmente o parâmetro $\Lambda=0$ nas equações (2.92) e (2.83). É possível observar que considerar artificialmente $\Lambda=0$ nas equações (2.92) e (2.83) significa desprezar o termo proporcional à $\phi$ nas expansões em série de Taylor referentes às condições de fronteira verticais representadas por (2.8), ou seja, significa supor a condição de fronteira $w\left(x, y, p_{0}, t\right)=w\left(x, y, p_{T}, t\right)=0$. Neste caso, a superfície e o topo da atmosfera são simplesmente representados pelas superfícies isobáricas $\mathrm{p}_{0}$ e $\mathrm{p}_{\mathrm{T}}$, respectivamente.

Tabela 4.6: Similar à Tabela 4.5, mas para os coeficientes de interação calculados com $\Lambda=0$ nas equações (2.92) e (2.83), ou seja, considerando como condições de fronteira na vertical $\mathrm{w}\left(\mathrm{x}, \mathrm{y}, \mathrm{p}_{0}, \mathrm{t}\right)=\mathrm{w}\left(\mathrm{x}, \mathrm{y}, \mathrm{p}_{\mathrm{T}}, \mathrm{t}\right)=0$.

\begin{tabular}{|l|l|l|l|l|l|l|l|l|l|}
\hline & $\mathrm{a}$ & $\mathrm{b}$ & $\mathrm{c}$ & $\mathrm{w}_{\mathrm{a}}$ & $\varpi_{\mathrm{b}}$ & $\mathrm{w}_{\mathrm{c}}$ & $\mathrm{i} \eta_{\mathrm{a}}{ }^{\mathrm{bc}}$ & $\mathrm{i} \eta_{\mathrm{b}}{ }^{\mathrm{ac}}$ & $\mathrm{i} \eta_{\mathrm{c}}{ }^{\mathrm{ab}}$ \\
\hline 1 & $0,4,2, \mathrm{R}$ & $1,4,0, \mathrm{M}$ & $1,0,1, \mathrm{R}$ & 0,8438 & 0,851 & 0 & $-0,463$ & 0,4335 & 0,00 \\
\hline 2 & $0,6,2, \mathrm{R}$ & $1,6,0, \mathrm{M}$ & $1,0,1, \mathrm{R}$ & 0,761 & 0,714 & 0 & 3,0242 & $-2,985$ & 0,00 \\
\hline 3 & $1,2,2, \mathrm{GO}$ & $1,1,1, \mathrm{GO}$ & $0,1,1, \mathrm{R}$ & 3,0112 & 2,3039 & 0,6489 & 0 & 0 & 0 \\
\hline 4 & $1,8,1, \mathrm{GO}$ & $1,1,1, \mathrm{GO}$ & $0,7,1, \mathrm{R}$ & 3,0497 & 2,3039 & 0,7717 & $-0,353$ & 0,1864 & 0,0021 \\
\hline 5 & $2,5,1, \mathrm{GO}$ & $2,1,0, \mathrm{M}$ & $0,4,2, \mathrm{R}$ & 1,7022 & 0,8905 & 0,83 & $-0,121$ & 0,0673 & 0,0321 \\
\hline 6 & $1,2,0, \mathrm{M}$ & $1,1,-1, \mathrm{~K}$ & $0,3,2, \mathrm{R}$ & 1,0747 & $-0,274$ & 0,8126 & $-1,349$ & 1,1588 & 0,1234 \\
\hline
\end{tabular}

A partir da comparação entre as Tabelas 4.5 e 4.6 é possível verificar que os termos não lineares nas fronteiras verticais resultantes do termo proporcional à $\phi \frac{\partial w}{\partial p}$ nas expansões em série de Taylor em (2.8) alteram o balanço de energia nas tríades ressonantes determinadas neste capítulo. Comparando as Tabelas 4.5 e 4.6 é possível verificar que, em geral, as tríades tornam-se menos conservativas ao desprezar o termo $\bar{\rho} \phi \frac{\partial w}{\partial p}$ nas expansões em série de Taylor referentes às condições de fronteira na vertical dadas por (2.8). Também é interessante notar que, uma vez que os termos não lineares nas fronteiras verticais em (2.92) resultantes dos 
termos do tipo $\bar{\rho} \phi \frac{\partial w}{\partial p}$ em (2.8) contêm produtos envolvendo $\phi$ e/ou a divergência horizontal associada aos modos, esses termos não lineares nas fronteiras verticais são mais importantes para as tríades que contém ondas de gravidade-inerciais. De fato, comparando as Tabelas 4.5 e 4.6 é possível observar que esses termos não lineares nas fronteiras verticais são mais importantes para a tríade 4, que contém dois modos de gravidade-inerciais e uma onda de Rossby. Nota-se claramente a partir das Tabelas 4.5 e 4.6 que a presença desses termos é fundamental para o balanço de energia para essa tríade. $\mathrm{Na}$ ausência desses termos não lineares, há uma super estimativa da projeção no modo a devida à interação entre os modos b e c e uma sub estimativa dos coeficientes de interação $\eta_{b}{ }^{a c}$ e $\eta_{c}{ }^{a b}$, como pode ser verificado a partir da comparação entre as Tabelas 4.5 e 4.6. Em particular, é interessante notar que a projeção no modo de Rossby externo (modo c) devida à interação entre as duas ondas de gravidade-inerciais internas é uma ordem de grandeza menor na ausência dos termos não lineares nas fronteiras verticais resultantes dos termos proporcionais à $\phi \frac{\partial w}{\partial p}$ em (2.8). Conseqüentemente, a tríade 4 composta por duas ondas de Poincaré internas e uma onda de Rossby externa é bem menos conservativa na ausência desses termos. Para as tríades que não contém ondas de gravidade-inerciais, esses termos não lineares adicionais nas fronteiras verticais tem um papel menos relevante para a dinâmica da troca de energia entre os modos envolvidos.

Dessa forma, os resultados dispostos nas Tabelas 4.5 e 4.6 mostram que os termos não-lineares nas fronteiras verticais resultantes dos termos do tipo $\bar{\rho} \phi \frac{\partial w}{\partial p}$ em (2.8) têm um papel significativo no balanço de energia nas tríades ressonantes que contém modos de gravidade-inerciais. Logo, os resultados sugerem que a correta especificação das condições de fronteira na vertical em modelos atmosféricos de previsão de tempo e clima é fundamental para a correta representação das interações não lineares nesses modelos. Conseqüentemente, os resultados apresentados nas Tabelas 4.5 e 4.6 também sugerem que a previsibilidade nesses modelos também pode estar intimamente ligada à correta representação dos processos físicos que ocorrem na superfície e no topo da atmosfera, tais como os fluxos turbulentos na camada limite planetária e os processos de transferência radiativa. 


\section{CAPÍTULO 5}

\section{Dinâmica das interações ressonantes}

Algumas possíveis interações ressonantes envolvendo uma onda de Rossby barotrópica e duas ondas equatoriais internas associadas ao mesmo modo baroclínico foram determinadas no capítulo anterior. No presente capítulo será analisada a dinâmica das trocas de energia entre as ondas envolvidas nessas tríades através de integrações das equações reduzidas (2.96) para alguns desses tripletos determinados.

Uma vez que os coeficientes de interação $\eta_{\mathrm{a}}{ }^{\mathrm{bc}}$ são imaginários puros ${ }^{3}$, o sistema de equações para uma tríade ressonante representado por (2.96) é um sistema Hamiltoniano, sendo $A_{j}$ e $A_{j}^{*}$, com j = a, b ou c, as variáveis canônicas conjugadas, sendo que os sub-índices a, b e c representam os três modos constituintes do tripleto ressonante, ou seja, a $=(m, k, n, r)$, onde $\mathrm{m}$ caracteriza a estrutura vertical, $\mathrm{k}$ é o número de onda em $\mathrm{x}, \mathrm{n}$ o modo meridional, que caracteriza a estrutura das autofunções em y, e r distingue os tipos de onda permitidos, como discutido anteriormente. A forma Hamiltoniana do sistema de equações (2.96) é escrita como segue:

$$
\begin{aligned}
& c_{a}{ }^{2} \frac{d A_{a}}{d \tau}=\eta_{a}^{b c} \frac{\partial H}{\partial A_{a}^{*}} \\
& c_{b}{ }^{2} \frac{d A_{b}}{d \tau}=\eta_{b}^{a c} \frac{\partial H}{\partial A_{b}^{*}} \\
& c_{c}{ }^{2} \frac{d A_{c}}{d \tau}=\eta_{c}^{a b} \frac{\partial H}{\partial A_{c}^{*}}
\end{aligned}
$$

onde $\mathrm{H}$ representa a Hamiltoniana do sistema, sendo escrita como

$$
H=2 \operatorname{Re}\left(A_{a} A_{b}^{*} A_{c}^{*}\right)
$$

\footnotetext{
${ }^{3} \mathrm{O}$ problema de três ondas ressonantes dado por (2.96) só é integrável se os coeficientes de interação $\eta_{\mathrm{a}}{ }^{\mathrm{bc}}, \eta_{\mathrm{b}}{ }^{\mathrm{ac}} \mathrm{e}$ $\eta_{\mathrm{c}}^{\mathrm{ab}}$ forem reais ou imaginários puros.
} 
Em (5.2), $\operatorname{Re}()$ representa a parte real do número complexo. A partir das equações (3.13), que governam a evolução temporal da energia dos modos numa determinada tríade ressonante, obtém-se as relações de Manley-Rowe:

$$
\frac{1}{i \eta_{a}^{b c}} \frac{d E_{a}}{d \tau}=\frac{1}{i \eta_{b}^{a c}} \frac{d E_{b}}{d \tau}=\frac{1}{i \eta_{c}^{a b}} \frac{d E_{c}}{d \tau}
$$

Assim, a partir das relações de Manley-Rowe (5.3) segue que o sistema (2.96) apresenta, além do termo de ordem dominante da energia total $\mathrm{E}^{(0)}$ e da Hamiltoniana $\mathrm{H}$, mais três grandezas conservativas, dadas por

$$
\frac{d}{d \tau}\left(\frac{E_{a}}{i \eta_{a}^{b c}}-\frac{E_{b}}{i \eta_{b}^{a c}}\right)=\frac{d}{d \tau}\left(\frac{E_{a}}{i \eta_{a}^{b c}}-\frac{E_{c}}{i \eta_{c}^{a b}}\right)=\frac{d}{d \tau}\left(\frac{E_{b}}{i \eta_{b}^{a c}}-\frac{E_{c}}{i \eta_{c}^{a b}}\right)=0
$$

Logo, como o sistema (2.96) possui três graus de liberdade e, pelo menos, três integrais primeiras, o problema de três ondas ressonantes representado por (2.96) ou (5.1) é integrável. Soluções analíticas para esse problema podem ser expressas em termos das funções elípticas de Jacobi, como será mostrado em seguida. Antes de obter a solução analítica de (2.96) é interessante observar primeiramente que este sistema de equações reduzidas pode também ser usado para a análise de estabilidade fracamente não linear de uma dada onda numa tríade ressonante. De fato, quando a amplitude de um dos modos, por exemplo a amplitude do modo a, é muito maior que as amplitudes dos modos $\mathbf{b}$ e c, as equações (2.96) podem ser aproximadas através de uma linearização em torno da amplitude do modo a, resultando no seguinte sistema linearizado:

$$
\begin{gathered}
\frac{d A_{a}}{d \tau}=0 \\
c_{b}{ }^{2} \frac{d A_{b}}{d \tau}=\eta_{b}^{a c} A_{a} A_{c}^{*} \\
c_{c}{ }^{2} \frac{d A_{c}}{d \tau}=\eta_{c}^{a b} A_{a} A_{b}^{*}
\end{gathered}
$$

De (5.5), segue que as amplitudes dos modos $\mathbf{a}, \mathbf{b}$ e $\mathbf{c}$ são governadas neste caso pelas seguintes equações: 


$$
\begin{aligned}
& A_{a}(t)=A_{a}(t=0)=\text { cons tante } \\
& c_{b}{ }^{2} \frac{d^{2} A_{b}}{d \tau^{2}}=-\frac{\eta_{b}^{a c} \eta_{c}^{a b}\left|A_{a}\right|^{2}}{c_{c}{ }^{2}} A_{b} \\
& c_{c}{ }^{2} \frac{d^{2} A_{c}}{d \tau^{2}}=-\frac{\eta_{b}^{a c} \eta_{c}^{a b}\left|A_{a}\right|^{2}}{c_{b}{ }^{2}} A_{c}
\end{aligned}
$$

De acordo com (5.6b) e (5.6c), como os coeficientes de interação são imaginários puros, observa-se que se os coeficientes $\eta_{\mathrm{b}}{ }^{\mathrm{ac}}$ e $\eta_{\mathrm{c}}{ }^{\mathrm{ab}}$ tiverem sinais opostos, as amplitudes dos modos b e c apresentam um comportamento oscilatório no tempo, com a freqüência de oscilação dada por $\frac{\left|A_{a}(0)\right|\left|\eta_{b}^{a c} \eta_{c}^{a b}\right|^{\frac{1}{2}}}{c_{b} c_{c}}$. Por outro lado, se os coeficientes de interação $\eta_{\mathrm{b}}{ }^{\mathrm{ac}}$ e $\eta_{\mathrm{c}}{ }^{\mathrm{ab}}$ tiverem o mesmo sinal, as amplitudes $A_{b}$ e $A_{c}$ crescem exponencialmente com a escala longa de tempo, com a taxa de crescimento proporcional à $\left|\mathrm{A}_{\mathrm{a}}(0)\right|$. Uma vez que a onda com a maior freqüência absoluta apresenta o coeficiente de interação com o sinal oposto ao dos outros dois modos constituintes do tripleto (e, conseqüentemente, com o maior valor absoluto), as amplitudes $A_{b}$ e $A_{c}$ permanecem pequenas comparadas com a amplitude do modo a exceto se o modo a apresentar a maior freqüência absoluta do tripleto ressonante. Neste caso, se $\left|\varpi_{\mathrm{a}}\right|>$ $\left|\varpi_{b, c}\right|$, o modo a é instável com relação às ondas b e c e age como uma fonte de energia para esses modos. Em problemas relacionados com física de plasmas, o modo a neste caso é comumente referido como "onda bomba" (Weiland e Wilhelmsson 1977), e a instabilidade produzida por essa onda é comumente referida como instabilidade paramétrica. Entretanto, quando as amplitudes dos modos b e c tornam-se comparáveis com a do modo a, a aproximação linear dada por (5.5) torna-se inválida. Logo, a aproximação dada por (5.5) no caso instável só é válida para um intervalo de tempo pequeno. De qualquer forma, a análise de estabilidade realizada através do sistema (5.5) mostra que a troca de energia envolvendo os modos num tripleto ressonante é fortemente favorecida se o modo de maior freqüência absoluta do tripleto detiver a maior parte da energia inicial. Em contrapartida, a troca de energia entre os modos $\mathbf{a}, \mathbf{b}$ e $\mathbf{c}$ numa tríade ressonante é fortemente inibida se a maior parte da energia inicial for projetada em um modo que não apresenta a maior freqüência absoluta do tripleto em questão.

Voltando às equações reduzidas completas (2.96) para as tríades ressonantes, é conveniente escrever as amplitudes $\operatorname{dos} \operatorname{modos} A_{j}(\tau), j=a, b$ ou $c$, na forma polar: 


$$
A_{j}(\tau)=R_{j}(\tau) e^{i \varphi_{j}(\tau)}
$$

onde $\mathrm{R}_{\mathrm{j}}(\tau)$ e $\varphi_{\mathrm{j}}(\tau)$ correspondem ao módulo e à fase de $\mathrm{A}_{\mathrm{j}}$, respectivamente. Substituindo (5.7) em (2.96), obtém-se um sistema de seis equações e seis incógnitas que governa a modulação na escala longa de tempo dos módulos e das fases das amplitudes das três ondas constituintes de um determinado tripleto ressonante. A partir dessas equações é possível mostrar que a troca de energia entre os modos $\mathbf{a}, \mathbf{b}$ e $\mathbf{c}$ de uma determinada tríade ressonante ocorre desde que $\sum_{j} \varphi_{j}(\tau) \neq \pi, \mathrm{j}=\mathrm{a}, \mathrm{b}$ e c , e é máxima se $\sum_{j} \varphi_{j}(\tau)=\frac{\pi}{2}$ (Domaracki e Loesch 1977). Logo, com o intuito de analisar o máximo potencial de troca de energia entre os modos no sistema, as fases das amplitudes $A_{j}$ dos modos serão fixadas de tal forma a satisfazer à condição $\sum_{j} \varphi_{j}(\tau)=\frac{\pi}{2}, \mathrm{j}=\mathbf{a}, \mathbf{b}$ e c. Neste caso, a evolução temporal dos módulos das amplitudes dos modos constituintes de um tripleto ressonante é governada pelo seguinte sistema:

$$
\begin{aligned}
& c_{a}{ }^{2} \frac{d R_{a}}{d \tau}=i \eta_{a}^{b c} R_{b} R_{c} \\
& c_{b}{ }^{2} \frac{d R_{b}}{d \tau}=i \eta_{b}^{a c} R_{a} R_{c} \\
& c_{c}{ }^{2} \frac{d R_{c}}{d \tau}=i \eta_{c}^{a b} R_{a} R_{b}
\end{aligned}
$$

Como discutido anteriormente, o sistema (5.8) acima é integrável, e a solução analítica desse sistema é conhecida em termos das funções elípticas de Jacobi. Detalhes da obtenção da solução geral do problema de três ondas ressonantes similar ao problema (5.8) para uma condição inicial arbitrária podem ser encontrados em Craik (1985, Capítulo 8) ou em Ripa (1981). No presente capítulo desta tese será suposto que a amplitude da componente de maior freqüência absoluta de uma tríade ressonante, que apresenta o coeficiente de interação com sinal oposto ao das outras duas ondas, seja nula no instante inicial. Isto não é necessário para se obter a solução analítica das equações (5.8) em termos das funções elípticas de Jacobi, mas torna a análise um tanto mais clara, além de não perder em generalidade, uma vez que, mesmo para uma condição inicial arbitrária, a solução também é escrita em termos dessas funções 
elípticas. Assim, é conveniente estabelecer daqui em diante que o modo a corresponde ao modo que apresenta o coeficiente de interação com sinal oposto ao dos outros dois membros da tríade e que o modo c apresenta o menor coeficiente de interação. Com isso, supondo que $\mathrm{R}_{\mathrm{a}}(\mathrm{t}=0)=0, \mathrm{R}_{\mathrm{b}}(\mathrm{t}=0)=\mathrm{R}_{\mathrm{b}}{ }^{0}$ e $\mathrm{R}_{\mathrm{c}}(\mathrm{t}=0)=\mathrm{R}_{\mathrm{c}}{ }^{0}$, a solução analítica de (5.8) é escrita da seguinte forma (ver McGoldrick 1965):

$$
\begin{aligned}
& R_{a}(t)=R_{b}^{0}\left(\left|\frac{\eta_{a}^{b c}}{\eta_{b}^{a c}}\right| \frac{c_{b}^{2}}{c_{a}^{2}}\right)^{\frac{1}{2}} \operatorname{sn}\left(\frac{\Xi}{\widetilde{m}}\right) \\
& R_{b}(t)=R_{b}^{0} \operatorname{cn}\left(\frac{\Xi}{\widetilde{m}}\right) \\
& R_{c}(t)=R_{c}^{0} d n\left(\frac{\Xi}{\widetilde{m}}\right)
\end{aligned}
$$

onde sn, cn e dn correspondem às funções elípticas de Jacobi. A definição dessas funções especiais, bem como suas propriedades, são encontradas em Abramowitz e Stegun (1964, Capítulo 16). $\mathrm{O}$ argumento $\Xi$ e o parâmetro $\tilde{m}$ dessas funções elípticas neste caso são dados por

$$
\begin{aligned}
& \Xi=R_{c}^{0}\left(\frac{\left|\eta_{a}^{b c} \eta_{b}^{a c}\right|}{c_{a}{ }^{2} c_{b}{ }^{2}}\right)^{\frac{1}{2}} \varepsilon t \\
& \tilde{m}=\frac{\eta_{c}^{a b}}{\eta_{b}^{a c}} \frac{c_{b}{ }^{2}}{c_{c}{ }^{2}} \frac{R_{b}^{0^{2}}}{R_{c}^{0^{2}}}
\end{aligned}
$$

É interessante observar a partir das equações (5.9) e (5.10) que a freqüência de oscilação da amplitude dos modos com o tempo depende das amplitudes iniciais dos modos b e c, sendo mais sensível à amplitude inicial do modo c. Logo, verifica-se a partir das equações (5.9) e (5.10) que o período das trocas de energia entre os modos pode ser arbitrariamente longo ou arbitrariamente curto, dependendo das amplitudes iniciais dos modos. Para $\tilde{m}=0$ $\left(\eta_{c}^{a b}=0\right)$, as funções sn e cn são dadas, respectivamente, pelas funções trigonométricas sen e cos (Abramowitz e Stegun 1964, Capítulo 16), e a solução das equações reduzidas (5.8) para uma determinada tríade ressonante é dada por 


$$
\begin{aligned}
& R_{a}(t)=R_{b}^{0}\left(\left|\frac{\eta_{a}^{b c}}{\eta_{b}^{a c}}\right| \frac{c_{b}{ }^{2}}{c_{a}{ }^{2}}\right)^{\frac{1}{2}} \operatorname{sen}(\Xi) \\
& R_{b}(t)=R_{b}^{0} \cos (\Xi) \\
& R_{c}(t)=R_{c}^{0}=\text { cons } \tan t e
\end{aligned}
$$

Neste caso, como pode ser observado a partir de (5.11), a amplitude do modo c não varia no tempo, ou seja, este modo não troca energia com as demais componentes do tripleto. A função desse modo neste caso é agir como um catalisador, possibilitando a troca de energia entre os modos a e b e controlando o período dessas trocas de energia por meio de sua amplitude inicial $\mathrm{R}_{\mathrm{c}}{ }^{0}$. Este é exatamente o caso dos tripletos ressonantes determinados no Capítulo 4 que envolvem ondas de Yanai associadas ao primeiro modo baroclínico e ondas de Rossby externas com modo meridional $\mathrm{n}=2$, ambas possuindo o mesmo número de onda zonal $\mathrm{k} \geq 2^{-1 / 2}$, e o modo geostrófico zonalmente simétrico $(\mathrm{k}=0)$ com a estrutura vertical referente ao primeiro modo baroclínico $\mathrm{m}=1$ e possuindo modo meridional $\mathrm{n}=1$, onde o modo geostrófico zonalmente simétrico apresenta freqüência temporal nula e, portanto, age como o modo catalisador. É interessante observar que nas interações triádicas ressonantes que envolvem um modo geostrófico zonalmente simétrico (que possuem autofreqüência nula e, portanto, com coeficiente de interação também nulo), o período da troca de energia entre os modos a e b depende somente da amplitude inicial do modo catalisador, não dependendo da energia inicial do modo b. Além disso, é interessante notar nas equações (5.11) e (5.10a) que a freqüência temporal da troca de energia entre os modos a e b apresenta uma dependência linear com relação à amplitude inicial do modo c. Com isso, embora os modos geostróficos zonalmente simétricos não trocam energia com os modos com os quais interagem ressonantemente, a amplitude inicial de um modo geostrófico zonalmente simétrico numa determinada tríade ressonante controla a escala de tempo da troca de energia entre os dois modos restantes nessa determinada tríade.

A Figura 5.1 mostra a evolução temporal da energia modal para a tríade 2 da Tabela 4.5, que é constituída por uma onda de Rossby barotrópica com número de onda zonal $6(\mathrm{k} \approx$ 0,942) e modo meridional $\mathrm{n}=2$, uma onda de Yanai com o mesmo número de onda zonal e referente ao primeiro modo baroclínico $(\mathrm{m}=1)$ e um modo geostrófico zonalmente simétrico $(\mathrm{k}=0)$ com modo meridional $\mathrm{n}=1$ e a estrutura vertical correspondente ao modo baroclínico $m=1$. A Figura 5.1a mostra a evolução temporal das energias $c_{j}^{2} R_{j}^{2}, j=a, b$ e c, dos modos 
dessa tríade para as amplitudes iniciais $\mathrm{R}_{\mathrm{b}}{ }^{0}=\mathrm{R}_{\mathrm{c}}{ }^{0}=2$, enquanto a Figura 5.1b mostra a mesma evolução temporal mas para as amplitudes iniciais dadas por $\mathrm{R}_{\mathrm{b}}{ }^{0}=2$ e $\mathrm{R}_{\mathrm{c}}{ }^{0}=1$.

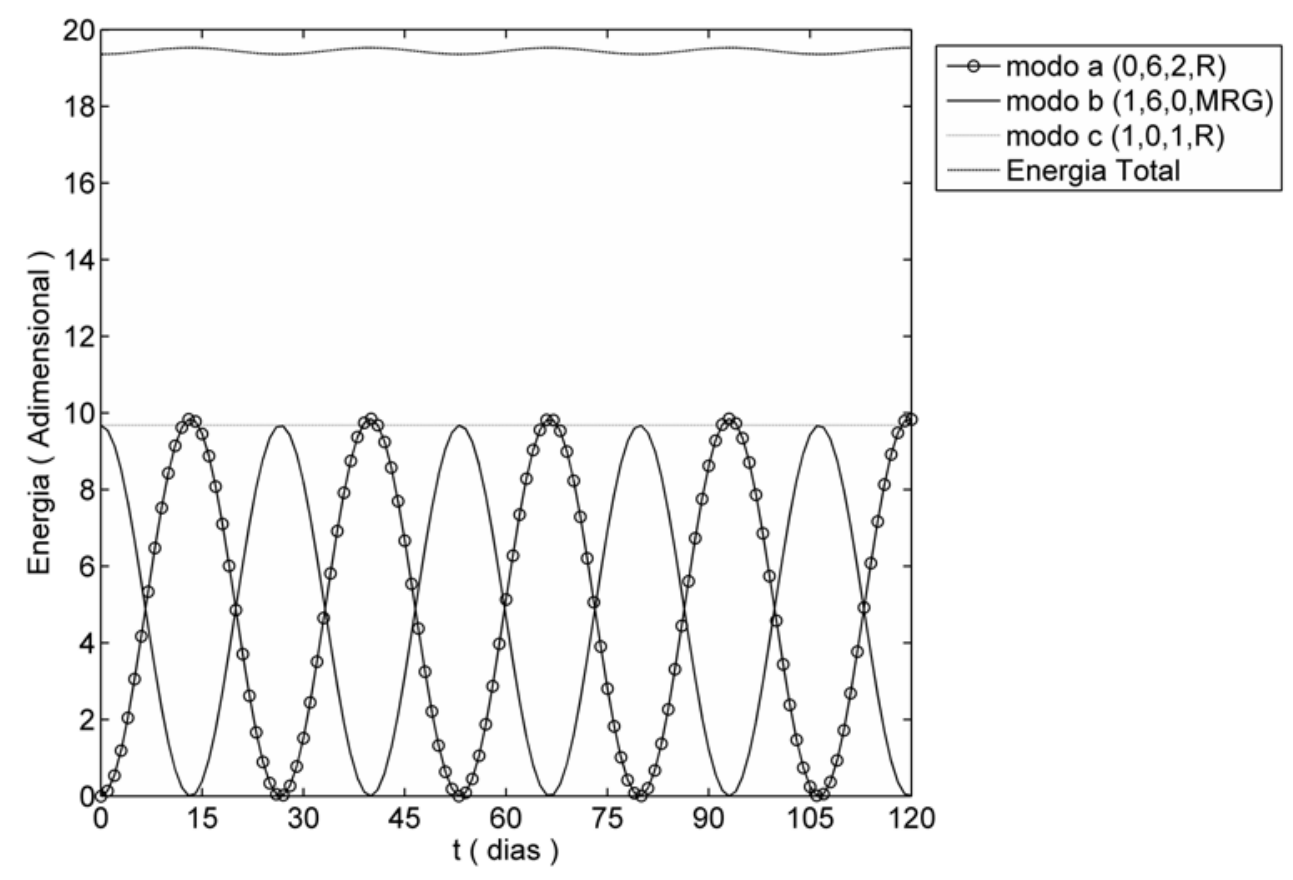

(a)

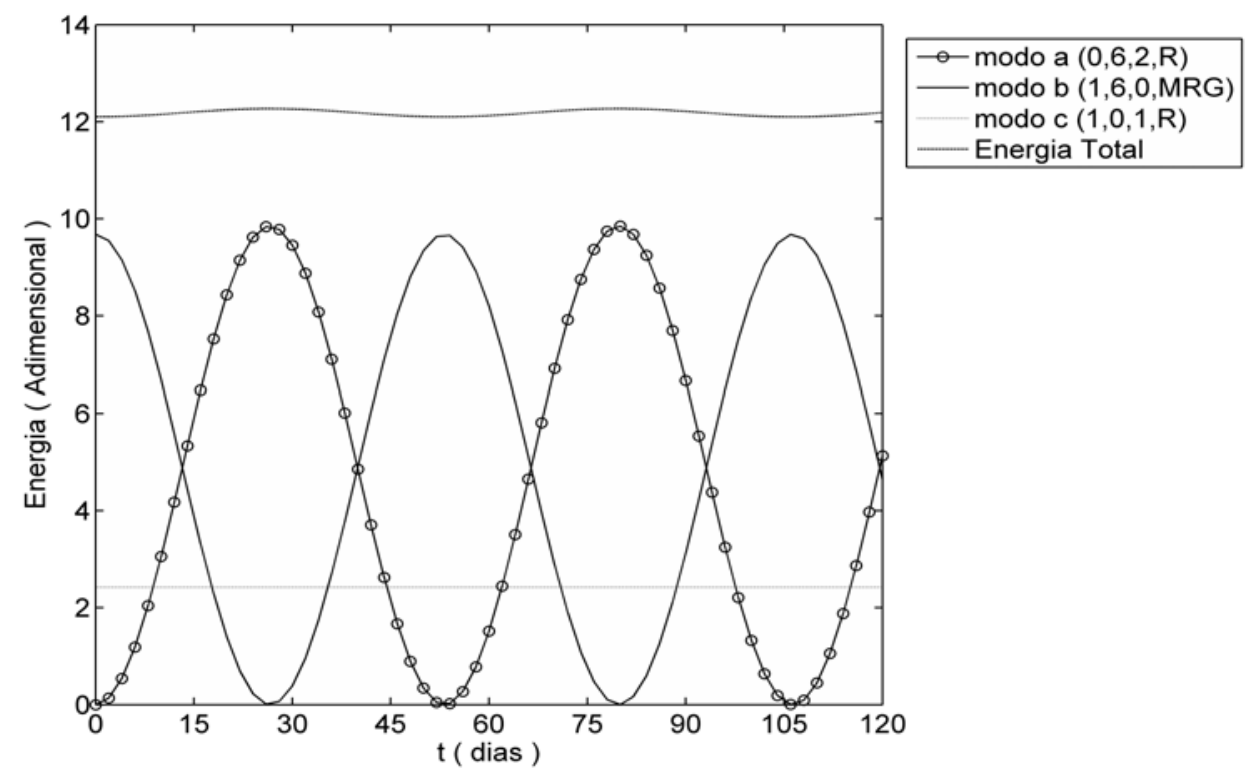

(b)

Figura 5.1: Evolução temporal da energia modal para a tríade constituída por: uma onda de Rossby barotrópica com número de onda zonal $6(\mathrm{k} \approx 0,942)$ e modo meridional $\mathrm{n}=2$ (modo 
a), uma onda de Yanai com o mesmo número de onda zonal e referente ao primeiro modo baroclínico $(\mathrm{m}=1)$ (modo b) e um modo geostrófico zonalmente simétrico $(\mathrm{k}=0)$ com modo meridional $\mathrm{n}=1$ e a estrutura vertical correspondente ao modo baroclínico $\mathrm{m}=1$ (modo $\mathbf{c}$ ). A figura mostra a evolução temporal da energia das ondas para a condição inicial caracterizada por: (a) $\mathrm{R}_{\mathrm{b}}{ }^{0}=\mathrm{R}_{\mathrm{c}}{ }^{0}=2$ e (b) $\mathrm{R}_{\mathrm{b}}{ }^{0}=2$ e $\mathrm{R}_{\mathrm{c}}{ }^{0}=1$.

Através da Figura 5.1 é possível verificar que, de fato, as interações ressonantes envolvendo as ondas mistas de Rossby-gravidade internas e as ondas de Rossby barotrópicas, ambas possuindo o mesmo número de onda zonal, e um modo geostrófico baroclínico zonalmente simétrico $(\mathrm{k}=0)$ e com modo meridional $\mathrm{n}=1$ são caracterizadas por uma troca de energia entre as ondas Yanai e as ondas de Rossby externas, enquanto o modo geostrófico permanece energeticamente inalterado no tempo, agindo apenas como o modo catalisador. Observa-se na Figura 5.1 que a onda de Rossby barotrópica e a onda de Yanai interna trocam energia entre si periodicamente, enquanto a energia total é conservada. Algumas pequenas oscilações na energia total são observadas na Figura. 5.1 e decorrem do fato de que a condição de ressonância para as freqüências temporais não é exatamente satisfeita devido à existência apenas de um conjunto discreto de modos normais, como discutido no Capítulo 4. É interessante observar que a onda de Rossby barotrópica, cuja energia é nula no instante inicial, é fortemente excitada e "retira" energia da onda mista de Rossby-gravidade interna, atingindo sua máxima energia possível, que corresponde à energia inicial da onda de Yanai. A onda de Yanai, por sua vez, atinge o mínimo de energia que corresponde à energia inicial do modo de Rossby. A partir de então a onda de Rossby passa a excitar a onda de Yanai interna e o ciclo se repete. Também é interessante observar na Figura 5.1 que o período da troca de energia entre as ondas de Rossby e de Yanai é de 27 dias na Figura 5.1a e de 54 dias na Figura 5.1b. Logo, quando a amplitude do modo catalisador é reduzida à metade, o período da troca de energia entre os dois modos restantes na tríade ressonante é dobrado, como previsto pela solução dada por (5.11). Assim, embora o modo geostrófico não troque energia com a onda de Rossby externa e a onda de Yanai interna na tríade 2 da Tabela 4.5, a energia inicial desse modo controla o período da troca de energia entre essas ondas, como verificado na Figura 5.1.

Um ponto importante a ser abordado acerca das interações ressonantes no modelo atmosférico aqui empregado refere-se às implicações das trocas de energia entre os modos constituintes de uma dada tríade ressonante para a solução de ordem dominante no espaço 
físico. Em outras palavras, surge uma interessante questão acerca das interações ressonantes: como as trocas regulares de energia entre as ondas constituintes de um tripleto ressonante se refletem na solução no espaço físico?

Para responder tal questão, as Figuras 5.2 a 5.4 destacam alguns aspectos da solução do problema de três ondas ressonantes (5.8) para a tríade referente à Figura 5.1 no espaço físico. Neste caso, os campos do vento e do geopotencial correspondentes à solução de ordem dominante do modelo aqui adotado são obtidos a partir das amplitudes $R_{j}(t), j=a, b$ e c, através da expansão espectral representada por (2.53) truncada de modo a considerar somente os três modos da tríade ressonante explorada na Figura 5.1. As Figuras 5.2 a 5.4 referem-se à solução correspondente à Figura 5.1b. A Figura 5.2 mostra um corte latitude-pressão da estrutura espacial da componente meridional do vento ao longo da longitude de $45^{\circ} \mathrm{W}$ em: (a) $\mathrm{t}=0,(\mathrm{~b}) \mathrm{t}=14$ dias, (c) $\mathrm{t}=28$ dias e (d) $\mathrm{t}=41$ dias.

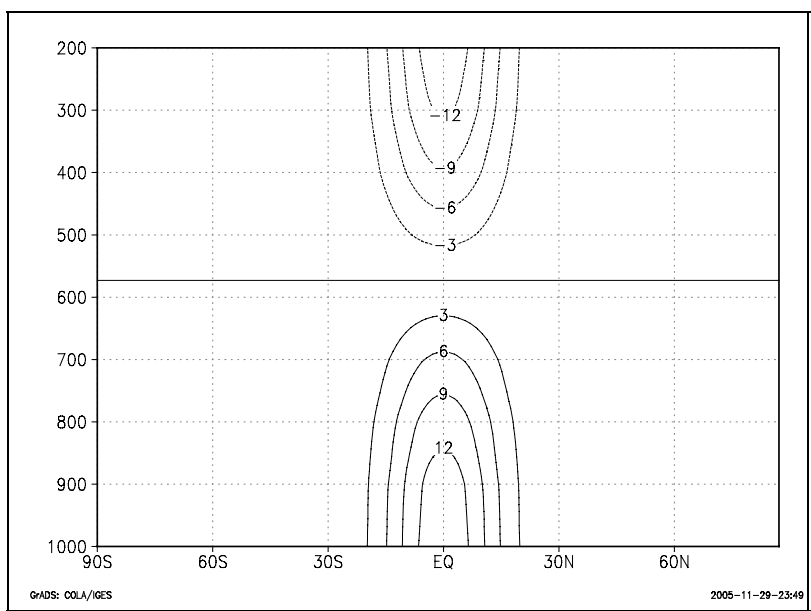

(a)

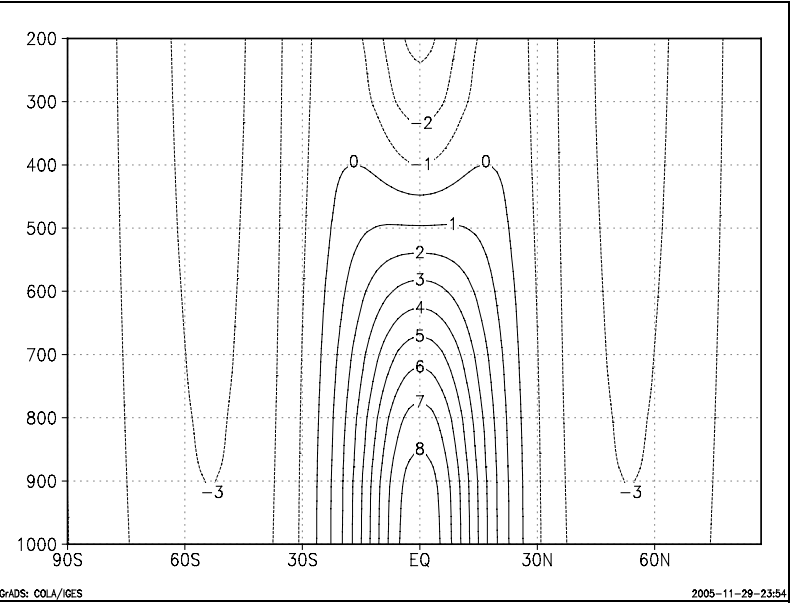

(b)

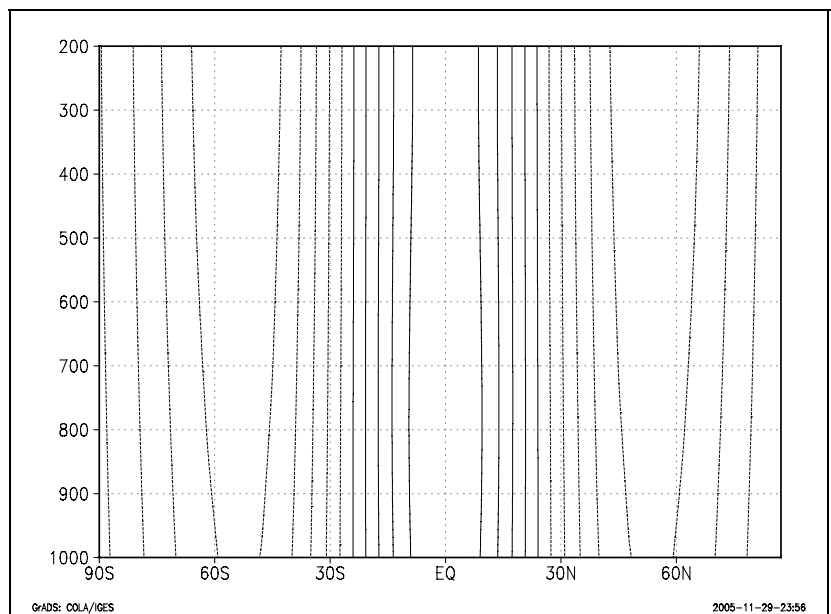

(c)

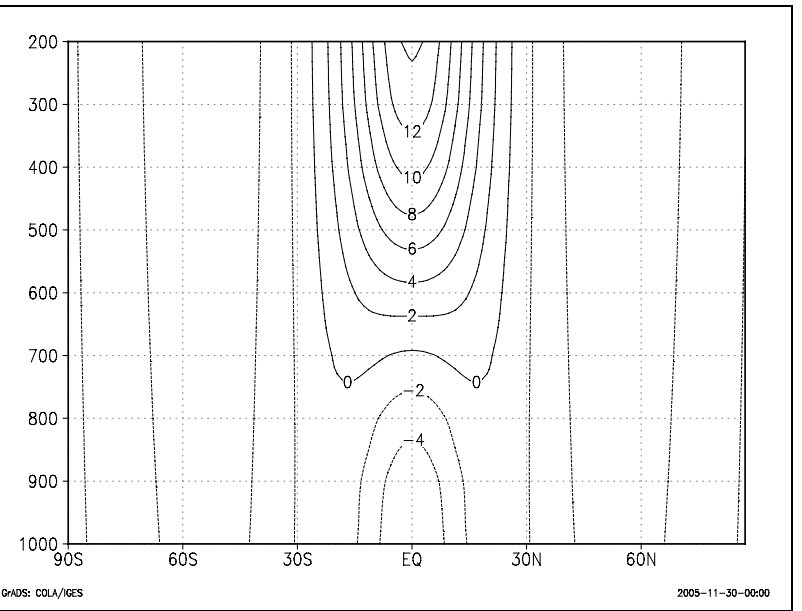

(d) 
Figura 5.2: Seção latitude-pressão da componente meridional do vento ao longo da longitude de $45^{\circ} \mathrm{W}$ em: (a) $\mathrm{t}=0$; (b) $\mathrm{t}=14$ dias; (c) $\mathrm{t}=28$ dias e (d) $\mathrm{t}=41$ dias. A latitude (na abscissa) é apresentada em graus, enquanto a pressão (na ordenada) é apresentada em $\mathrm{hPa}$. A componente meridional do vento corresponde à solução de ordem dominante do modelo de equações (2.1) truncada de modo a considerar somente as três ondas ressonantes constituintes da tríade 2 da Tabela 4.5. O campo de v é mostrado na figura acima na escala dimensional $(\mathrm{m} / \mathrm{s})$, usando a escala (2.11) para $\mathrm{U}=5 \mathrm{~m} / \mathrm{s}$.

Uma vez que a componente meridional do vento é nula para os modos geostróficos zonalmente simétricos, esta variável é bastante útil para analisar as implicações físicas da troca de energia entre a onda mista de Rossby-gravidade e a onda de Rossby externa mostrada na Figura 5.1. Como estas duas ondas são caracterizadas por um modo meridional par, o campo de $\mathrm{v}$ é simétrico com relação ao equador para esses dois modos, ao passo que os campos de u e $\phi$ são anti-simétricos. Logo, a estrutura espacial desses modos é caracterizada por vórtices centrados no equador, com a onda de Rossby externa apresentando uma estrutura mais oscilatória em y, com dois zeros para o campo de $\mathrm{v}(\mathrm{y})$ e três zeros para $\mathrm{u}(\mathrm{y})$ e $\phi(\mathrm{y})$ (um deles no equador), enquanto a onda mista de Rossby-gravidade possui os campos de u(y) e $\phi(y)$ com apenas um único zero (no equador) e com a componente meridional máxima ao longo do equador e estritamente confinada ao longo do mesmo (sem apresentar nenhuma oscilação em y) (ver equação 2.45). Inclusive, a intensa componente meridional do vento no equador é o fator que melhor caracteriza a onda mista de Rossby-gravidade e tem sido extensivamente usada em estudos observacionais para identificar o sinal dessa onda na atmosfera tropical (Takayabu 1994; Pires et. al. 1997; Santos et al. 2002). Um outro aspecto importante acerca das auto-soluções do problema de $\mathrm{O}(1)$ do modelo aqui adotado é que as autofunções $\xi_{\mathrm{a}}(\mathrm{y})$ correspondentes ao modo externo apresentam uma considerável projeção em latitudes médias e altas, não sendo confinadas equatorialmente. Em contrapartida, as autofunções $\xi_{\mathrm{a}}(\mathrm{y})$ associadas aos modos internos são bastante confinadas na região tropical, como mostrado no Capítulo 2. De fato, em $\mathrm{t}=0$ a amplitude da onda de Rossby barotrópica é nula e, conseqüentemente, a Figura 5.2a mostra que nesse instante inicial a componente meridional do vento é estritamente confinada equatorialmente e apresenta a estrutura vertical mostrada na Figura 2.2b, com o sinal mais forte na superfície e na alta troposfera e com a inversão de fase ocorrendo exatamente na média troposfera (entre $600 \mathrm{hPa}$ e $500 \mathrm{hPa}$ ). Em t $=$ 
14 dias, pode ser observado através da Figura 5.1b que a onda de Rossby externa e a onda de Yanai apresentam energias praticamente iguais. Como resultado, a Figura 5.2b mostra que, nesse instante, ambos os modos contribuem quase em igualdade de proporções para a componente meridional do vento. É interessante notar na Figura 5.2b a manifestação da onda de Rossby externa através do centro de ação em aproximadamente $60^{\circ}$ de latitude (Sul e Norte) com estrutura aproximadamente barotrópica. Na região equatorial, nesse instante de tempo $(\mathrm{t}=14$ dias $)$ a superposição das atividades da onda de Rossby barotrópica e da onda mista interna resulta no confinamento da solução na baixa troposfera. Já em $t=41$ dias, a Figura 5.2d mostra que a superposição das atividades dos modos de Rossby barotrópico e de Yanai baroclínico, que também contribuem quase igualmente para a componente meridional do vento (ver Figura 5.1b), resulta no confinamento da solução na alta troposfera. O confinamento da solução na alta ou na baixa troposfera depende da fase relativa entre os modos verticais que se superpõem. A partir da Figura 2.2 pode ser observado que se o modo barotrópico e o primeiro modo baroclínico estão em fase, a solução tende a ser confinada na baixa troposfera, enquanto se esses mesmos modos verticais estiverem exatamente em oposição de fase, por exemplo, a solução torna-se confinada na alta troposfera. Em $\mathrm{t}=28$ dias, a Figura 5.2c mostra que a componente meridional do vento é totalmente resultante da atividade da onda de Rossby barotrópica, posto que a energia da onda de Yanai é quase nula neste instante de tempo (Figura 5.1b). É interessante observar na Figura 5.2c a considerável amplitude da componente meridional do vento associada à onda de Rossby externa constituinte da tríade 2 da Tabela 4.5 em latitudes médias e altas. Nota-se nesta figura a extensão da influência dessa onda até quase $90^{\circ}$ de latitude, com um centro de ação em aproximadamente $60^{\circ}$.

A Figura 5.3 ilustra a distribuição horizontal dos campos do vento horizontal e do geopotencial em $1000 \mathrm{hPa}$, associados à mesma solução das Figuras $5.1 \mathrm{~b}$ e 5.2 , em $\mathrm{t}=0$ (Fig. 5.3a) e em $\mathrm{t}=28$ dias (Fig. 5.3b). Em $\mathrm{t}=0$, a estrutura espacial dos campos do vento horizontal e do geopotencial dispostos na Figura 5.3a é devida à superposição das atividades da onda de Yanai e do modo geostrófico zonalmente simétrico, ambos com a estrutura vertical do primeiro modo baroclínico. De fato, nota-se claramente na Figura 5.3a que o campo do vento horizontal é caracterizado por uma componente zonal de leste que independe da longitude e é máxima no equador. O campo do geopotencial associado a este modo puramente geostrófico é caracterizado por dois máximos positivos em aproximadamente $15^{\circ} \mathrm{S}$ e $15^{\circ} \mathrm{N}$. Conseqüentemente, há uma interferência construtiva (destrutiva) no campo do geopotencial entre o modo geostrófico zonalmente simétrico e a onda mista de Rossby-gravidade nas 
regiões das cristas (cavados) referentes às ondas de Yanai, resultando no padrão observado na Figura 5.3a, com o geopotencial apresentando uma seqüência alternada de cristas reforçadas e nodos.

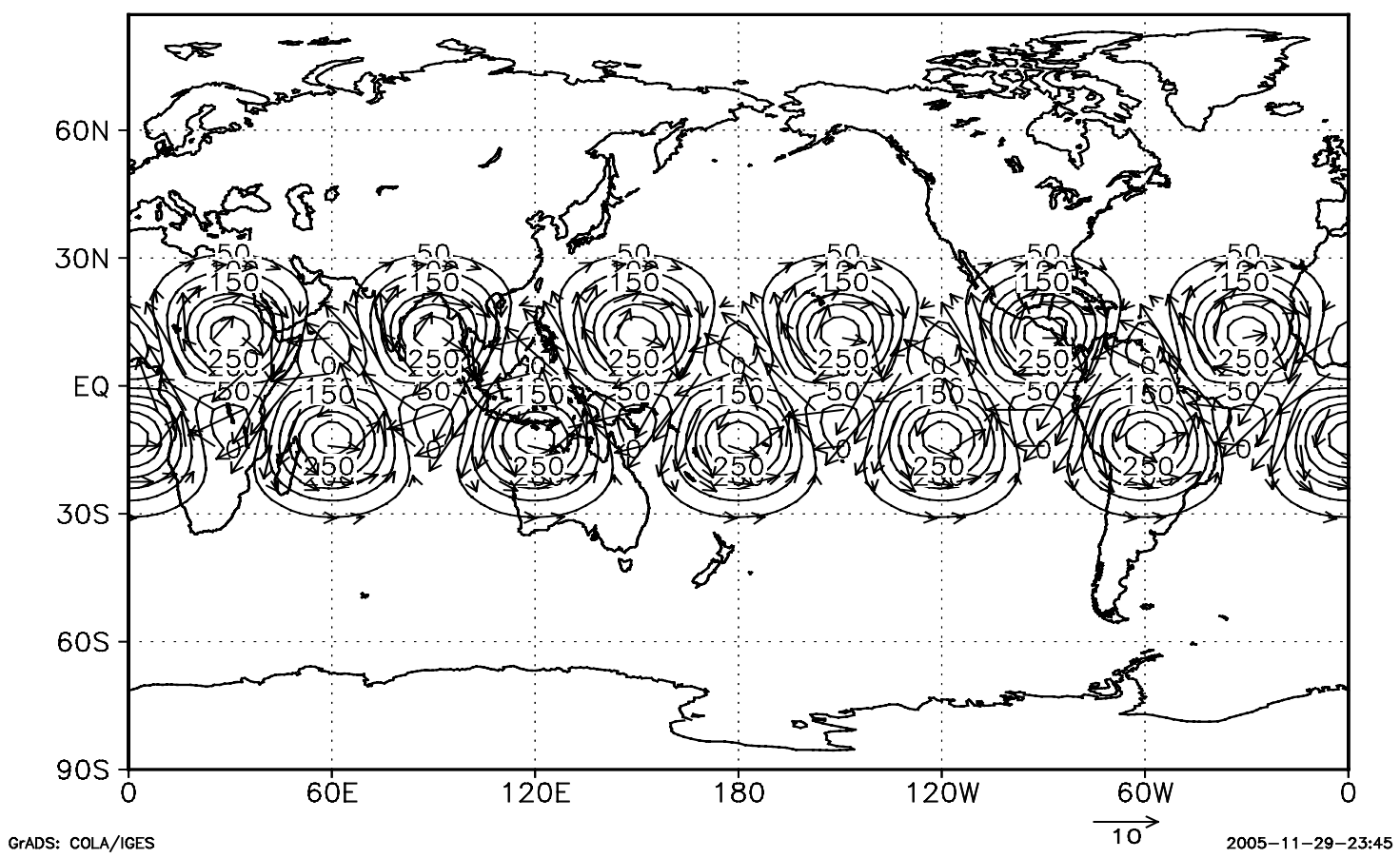

(a)

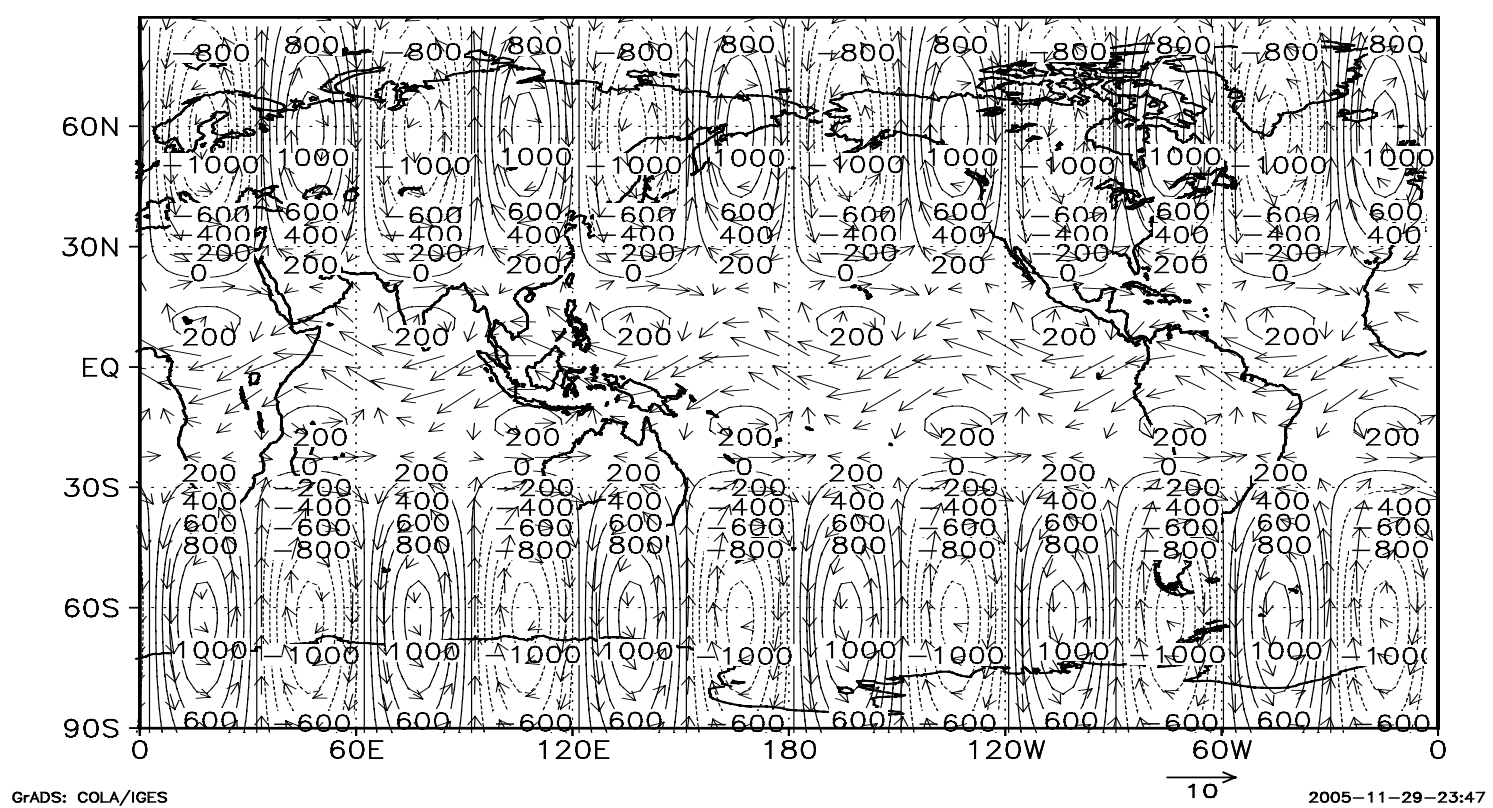

(b)

Figura 5.3: Distribuição horizontal dos campos do vento (vetor) e do geopotencial (contornos) em $\mathrm{p}=1000 \mathrm{hPa}$ associados à mesma solução das Figuras $5.1 \mathrm{~b}$ e $5.2 \mathrm{em}$ : (a) $\mathrm{t}=0$; (b) $\mathrm{t}=28$ 
dias. Os campos do vento horizontal e do geopotencial são dados, respectivamente, $\mathrm{em} \mathrm{m} / \mathrm{s} \mathrm{e}$ em $\mathrm{m}^{2} \mathrm{~s}^{-2}$, usando as escalas dadas por (2.11) para $\mathrm{U}=5 \mathrm{~m} / \mathrm{s}, \beta=2,3 \times 10^{-11} \mathrm{~m}^{-1} \mathrm{~s}^{-1} \mathrm{e} \mathrm{L}=$ $10^{6} \mathrm{~m}$.

O mesmo ocorre para a componente zonal do vento nessas latitudes de $\pm 15^{\circ}$. Os vórtices centrados ao longo do equador, resultantes da atividade da onda mista de Rossby-gravidade, também são claramente notados na Figura 5.3a. Em $\mathrm{t}=28$ dias, a Figura 5.3b mostra a estrutura espacial dos campos do vento horizontal e do geopotencial resultantes da atividade da onda de Rossby externa e do modo geostrófico zonalmente simétrico baroclínico, uma vez que a energia da onda de Yanai é quase nula nesse instante (Figura 5.1b). A componente zonal de leste zonalmente simétrica do campo do vento na região equatorial também é notável na Figura 5.3b, assim como os vórtices centrados no equador que são característicos dos modos de Rossby com modo meridional par. Também é interessante notar na Figura 5.3b que os gradientes de pressão na região equatorial associados ao modo de Rossby externo são pequenos. O gradiente de pressão pequeno na região equatorial é uma característica dos modos de Rossby com modo meridional $\mathrm{n}>1$, que também apresentam uma maior amplitude do campo do geopotencial em latitudes mais altas (Matsuno 1966; Silva Dias e Schubert 1979; Raupp 2002). De fato, observa-se na Figura 5.3b uma seqüência alternada de centros de ação ciclônicos e anticiclônicos (com número de onda zonal 6), com um notável balanço geostrófico entre os campos do vento e do geopotencial, em $\pm 60^{\circ}$ de latitude. Estes centros de ação são resultantes da onda de Rossby barotrópica referente à tríade 2 da Tabela 4.5.

A Figura 5.4 mostra a evolução temporal da divergência horizontal no nível de $200 \mathrm{hPa}$ em $\left(45^{\circ} \mathrm{W}, 12^{\circ} \mathrm{S}\right)$ (Figura 5.4a) e da componente meridional do vento no nível de $500 \mathrm{hPa}$ em $\left(45^{\circ} \mathrm{W}, 60^{\circ} \mathrm{S}\right)$ (Figura $\left.5.4 \mathrm{~b}\right)$. 


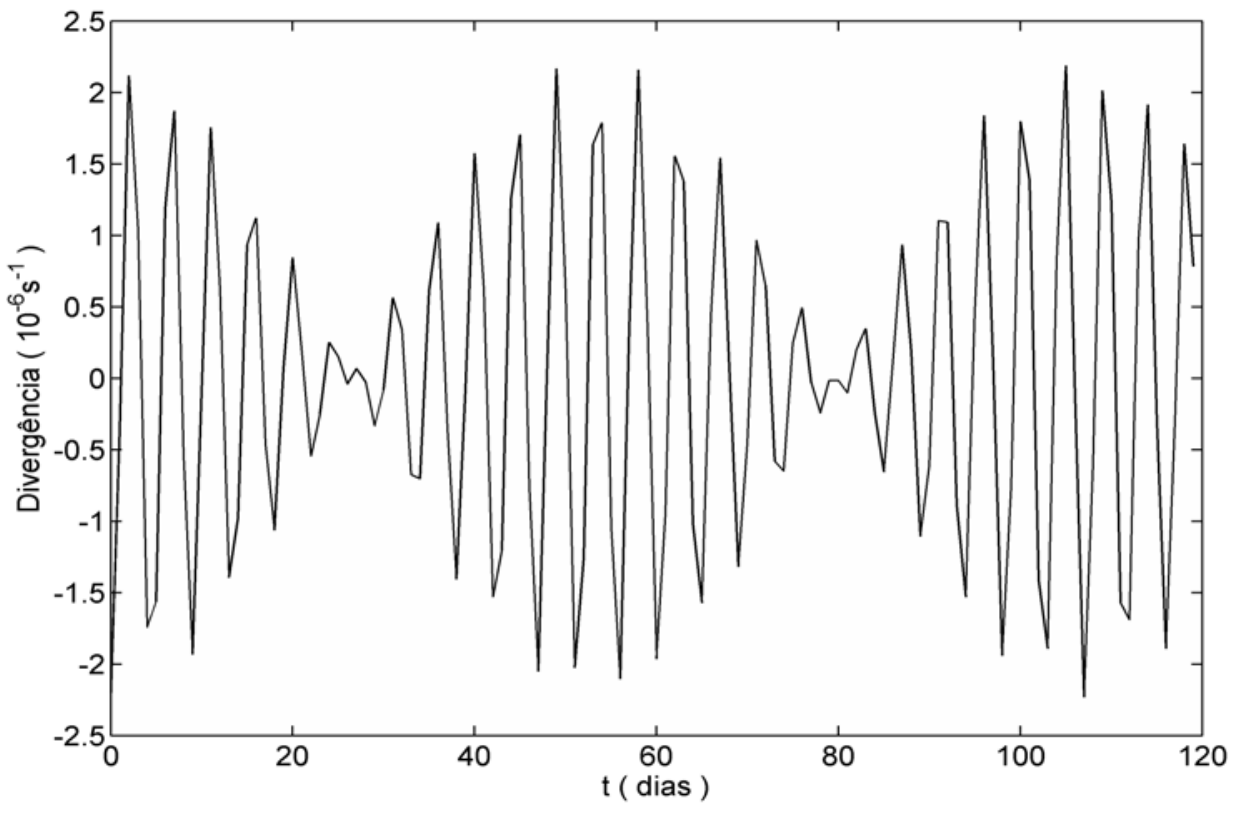

(a)

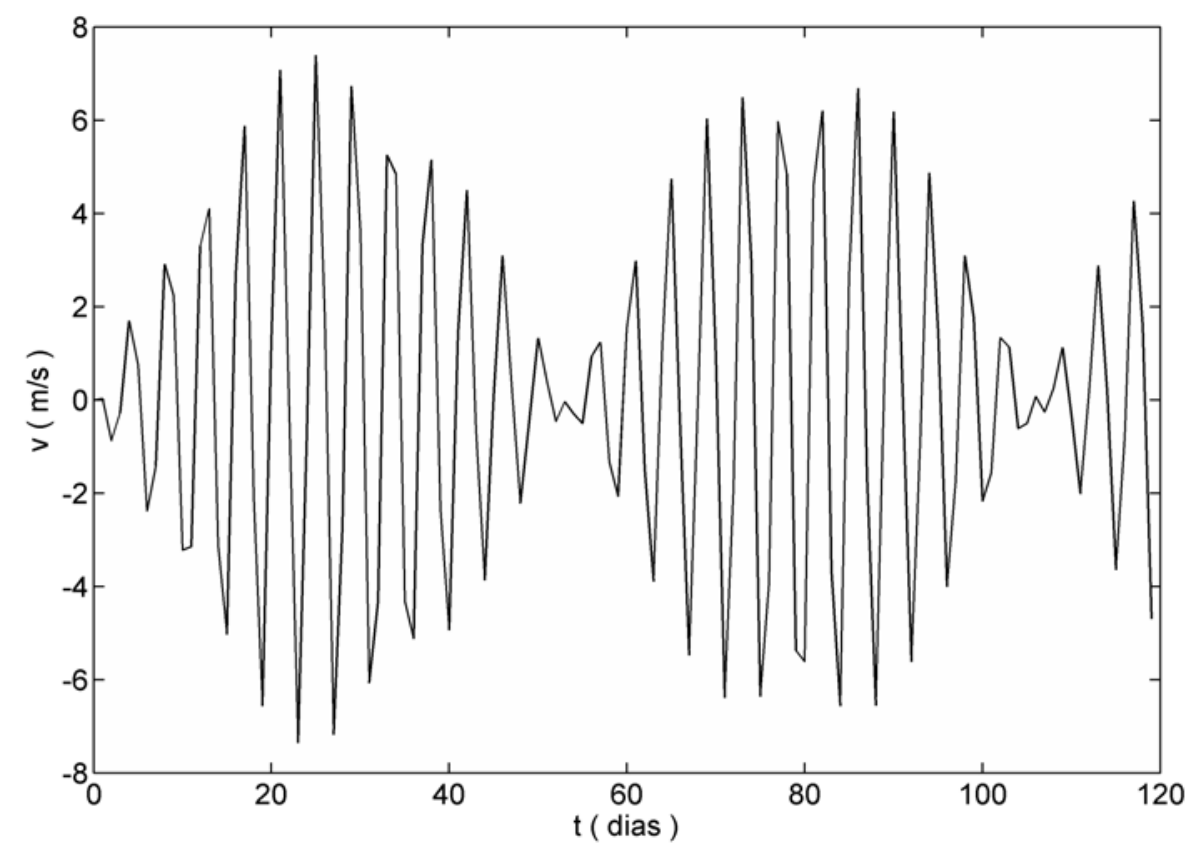

(b)

Figura 5.4: Evolução temporal da divergência horizontal no nível de $200 \mathrm{hPa}$ em $\left(45^{\circ} \mathrm{W}, 12^{\circ} \mathrm{S}\right)$ (a) e da componente meridional do campo do vento no nível de $500 \mathrm{hPa}$ em $\left(45^{\circ} \mathrm{W}, 60^{\circ} \mathrm{S}\right)$ (b) associadas à solução referente às Figuras 5.1b, 5.2 e 5.3. Os campos de v e $\left(\frac{\partial u}{\partial x}+\frac{\partial \mathrm{v}}{\partial y}\right)$ são dados, respectivamente, em $\mathrm{m} / \mathrm{s}$ e em $10^{-6} \mathrm{~s}^{-1}$, usando as escalas dadas por (2.11) para $\mathrm{U}=$ $5 \mathrm{~m} / \mathrm{s}, \beta=2,3 \times 10^{-11} \mathrm{~m}^{-1} \mathrm{~s}^{-1} \mathrm{e} \mathrm{L}=10^{6} \mathrm{~m}$. 
A evolução temporal da divergência horizontal e da componente meridional do vento ilustrada na Fig. 5.4 mostra a existência de oscilações locais com um período da ordem de 4 dias. Estas oscilações estão associadas à propagação de fase das ondas de Yanai e de Rossby barotrópica constituintes da tríade 2 da Tabela 4.5, dado que ambos os modos apresentam uma propagação de fase para oeste com um período dessa ordem. A modulação de freqüência mais baixa na amplitude destas oscilações locais está diretamente relacionada à modulação da energia dessas duas ondas, ou seja, é decorrente da troca de energia entre esses dois modos ressonantes. Isto pode ser claramente notado ao comparar a Figura $5.4 \mathrm{com}$ a Figura 5.1b. Os instantes em que a divergência horizontal do vento na Figura 5.4a é máxima correspondem exatamente aos instantes em que a energia da onda de Yanai (modo b) é máxima. Por outro lado, a mesma comparação entre as Figuras $5.1 \mathrm{~b}$ e $5.4 \mathrm{a}$ mostra que os instantes nos quais a divergência horizontal do vento na Figura 5.4a é mínima correspondem exatamente aos instantes em que a energia da onda de Rossby externa (modo a) é máxima (e, conseqüentemente, a energia do modo b é mínima). Da mesma maneira, os instantes nos quais a energia da onda de Rossby externa (modo a) é máxima correspondem exatamente aos instantes em que a componente meridional do vento em 500hPa na Figura 5.4b é máxima, e vice-versa. De fato, a partir da Figura 5.3 pode ser observado que a divergência horizontal associada à onda mista de Rossby-gravidade é máxima exatamente em $\pm 12^{\circ}$ de latitude e que a componente meridional do vento associada à onda de Rossby barotrópica apresenta uma considerável amplitude ao longo da latitude de $\pm 60^{\circ}$. Da mesma maneira, a divergência horizontal das ondas de Rossby barotrópicas é pequena e, uma vez que as ondas associadas aos modos baroclínicos são bastante confinadas equatorialmente, a onda de Yanai interna (modo b) apresenta uma amplitude muito pequena nas regiões de latitudes médias e altas ${ }^{4}$.

Logo, as trocas periódicas de energia entre as ondas constituintes de um trio ressonante implicam em periódicas mudanças de regime na solução no espaço físico. Tais mudanças de regime, por sua vez, ocorrem numa escala de tempo maior em relação ao período das oscilações locais decorrentes da própria propagação das ondas. Esta mudança de regime da solução no espaço físico devida à própria dinâmica interna do modelo é conhecida como vacilação (Lorenz 1963). Como observado nas Figuras 5.2 a 5.4, a condição inicial correspondente à solução mostrada na Figura 5.1 b reproduz uma magnitude típica de

\footnotetext{
${ }^{4}$ Além disso, como a autofunção da equação da estrutura vertical correspondente ao primeiro modo baroclínico é nula em aproximadamente $500 \mathrm{hPa}$, as variáveis dispostas neste nível de pressão representam essencialmente o sinal das perturbações decorrentes da atividade das ondas associadas ao modo barotrópico. Por esta razão, este nível é comumente referido como nível de equivalência barotrópica.
} 
anomalias de vento e geopotencial observada nos modelos numéricos de previsão de tempo e clima. Conseqüentemente, para as amplitudes iniciais dos modos caracterizando magnitudes típicas das perturbações no escoamento atmosférico, observa-se que a onda de Rossby barotrópica e a onda mista de Rossby-gravidade interna constituintes da tríade 2 da Tabela 4.5 trocam energia entre si na escala intra-sazonal, com o período da troca de energia sendo linearmente dependente da amplitude inicial do modo geostrófico baroclínico zonalmente simétrico constituinte dessa mesma tríade.

Com isso, os resultados dispostos nas Figuras 5.1 a 5.4 sugerem que a troca catalítica de energia entre a onda de Yanai interna e a onda de Rossby barotrópica por meio do modo geostrófico zonalmente simétrico observada na Figura 5.1, assim como as periódicas mudanças de regime na solução no espaço físico resultantes dessa troca, podem ter algumas implicações importantes para a variabilidade intra-sazonal da circulação atmosférica de grande-escala. Num modelo atmosférico mais realista, que inclui a parametrização dos processos convectivos úmidos, a divergência horizontal na alta troposfera na região equatorial está diretamente relacionada com a liberação de calor latente resultante da atividade convectiva. De acordo com a Figura 5.4, a divergência horizontal resultante da atividade da onda mista de Rossby-gravidade interna apresenta uma modulação na escala intra-sazonal em decorrência da troca de energia com a onda de Rossby barotrópica. A circulação atmosférica em médias e altas latitudes resultante da atividade da onda de Rossby barotrópica, por sua vez, também apresenta a mesma modulação de baixa freqüência resultante desse acoplamento ressonante, com as amplitudes das oscilações no campo da divergência no equador e da circulação em altas latitudes estando em oposição de fase. Logo, os resultados dispostos nas Figuras 5.1 a 5.4 sugerem que as trocas de energia entre as ondas mistas de Rossby-gravidade referentes ao primeiro modo baroclínico e as ondas de Rossby externas, ambas com o mesmo número de onda zonal, por meio do modo geostrófico zonalmente simétrico com modo meridional $\mathrm{n}=1$ e a mesma estrutura vertical da onda mista podem constituir uma possível fonte de variabilidade intra-sazonal para a circulação atmosférica de grande-escala, tanto nos trópicos quanto nos extratrópicos.

A onda mista de Rossby-gravidade discutida nas Figuras 5.1 a 5.4 apresenta um comprimento de onda da ordem de $6.000 \mathrm{Km}$. Da mesma maneira, a onda mista de Rossbygravidade constituinte da tríade ressonante 1 da Tabela 4.5 apresenta um comprimento de onda da ordem de $10.000 \mathrm{Km}$. Santos et al. (2002), usando dados da reanálise do NCEPNCAR durante o período do ABLE 2-b (Amazon Boundary Layer Experiment), obtiveram uma evidência observacional do sinal das ondas mistas de Rossby-gravidade na troposfera 
com escalas espaciais de 10.000-5.000Km. A Figura 5.5, retirada de Santos et al. (2002), ilustra o espectro de potência da componente meridional do vento no equador no nível de 850hPa ao longo do setor que engloba o Atlântico Equatorial e a Amazônia. Uma vez que a componente meridional do vento no equador é fator que melhor caracteriza a atividade das ondas mistas de Rossby-gravidade, a Figura 5.5 destaca o expressivo sinal das perturbações decorrentes da atividade das ondas de Yanai com comprimentos de onda de 5.000-10.000Km e períodos da ordem de 4-6 dias sobre o setor equatorial da América do Sul e do Atlântico. Um espectro similar ao da Figura 5.5 foi obtido por Pires et al. (1997) para o setor do Pacífico Equatorial usando quatro meses de dados do experimento TOGA COARE (Tropical Ocean Global Atmosphere - Coupled Ocean-Atmosphere Response Experiment). Santos et al. mostram que o padrão anti-simétrico em relação ao equador da divergência horizontal associada a estas ondas mistas de Rossby-gravidade tem um papel importante na propagação para oeste de distúrbios convectivos ao longo do Atlântico equatorial. Ao atingir a região da Amazônia, tais distúrbios convectivos estabelecem um significativo controle na precipitação sobre esta região. Evidências observacionais dessas ondas de Yanai troposféricas também foram obtidas por Takayabu (1994) e Wheeler e Kiladis (1999), que também destacam o significativo acoplamento dessas ondas com a convecção nos trópicos. O mecanismo troposférico de excitação dessas ondas é ainda bastante discutível. Um grande esforço por parte da comunidade científica tem sido aplicado na tentativa de compreender o mecanismo de excitação destes modos na atmosfera tropical. Alguns estudos explicam a geração destes modos através da teoria do forçamento lateral, proposta inicialmente por Mak (1969) (Mak 1969; Randel 1992; Magaña e Yanai 1995). De acordo com esta teoria, as ondas mistas de Rossby-gravidade são excitadas através da penetração nos trópicos de distúrbios ondulatórios provindos de médias latitudes. Uma outra hipótese comumente encontrada na literatura para a explicação do mecanismo de excitação da onda mista de Rossby-gravidade observada nos trópicos é baseada na teoria Wave-CISK (instabilidade condicional de segunda espécie) proposta inicialmente por Hayashi (1970) e posteriormente modificada por Hayashi e Sumi (1986) e Lau e Peng (1987) com a incorporação de efeitos não lineares na parametrização do aquecimento diabático. Esta última teoria propõe que a interação entre as ondas equatoriais e a convecção úmida gera modos instáveis com estruturas espaciais similares às das ondas mistas de Rossby-gravidade. Recentemente, Raupp e Silva Dias (2005) propuseram um possível mecanismo alternativo para a excitação e manutenção desses modos na atmosfera tropical baseado nas interações não lineares entre as ondas equatoriais no modelo de águarasa. Raupp e Silva Dias resolveram as equações da água-rasa no plano $\beta$-equatorial 
utilizando o método espectral com a base constituída pelas autofunções das equações linearizadas, dadas pelas próprias auto-soluções $(2.45),(2.50)$ e $(2.51)$ com a constante de separação fixa dada por $\mathrm{c}_{\mathrm{a}}=50 \mathrm{~m} / \mathrm{s}$, representativa do primeiro modo baroclínico, com uma forçante especificada no campo da massa representativa do aquecimento diabático resultante da típica atividade convectiva sobre a região da Amazônia durante o período de verão austral. Raupp e Silva Dias sugerem que as ondas mistas de Rossby-gravidade podem ser excitadas através de uma instabilidade das ondas longas de Rossby ressonantemente excitadas pela forçante no campo da massa.

Dessa forma, os resultados aqui apresentados destacam que as ondas mistas de Rossby-gravidade baroclínicas tipicamente observadas na atmosfera tropical também podem ser excitadas através do acoplamento ressonante com as ondas de Rossby barotrópicas por meio do modo geostrófico zonalmente simétrico com a estrutura vertical do primeiro modo baroclínico e modo meridional $\mathrm{n}=1$, sugerindo um outro possível mecanismo alternativo. Assim, as ondas mistas de Rossby-gravidade tipicamente observadas na atmosfera tropical, além de exercerem um significativo controle na atividade convectiva nos trópicos, devido ao acoplamento ressonante com as ondas de Rossby barotrópicas por meio do estado básico ${ }^{5}$, podem ter um importante papel na conexão entre as circulações atmosféricas tropical e extratropical. Neste contexto, de acordo com os resultados mostrados nas Figuras 5.1 a 5.4, uma vez que os padrões de teleconexões trópicos-extratrópicos observados na atmosfera são devidos às ondas de Rossby barotrópicas, a troca de energia entre as ondas mistas de Rossbygravidade e as ondas de Rossby externas por meio do estado básico podem implicar numa modulação desses padrões na escala de tempo intra-sazonal.

\footnotetext{
${ }^{5}$ Como os modos geostróficos zonalmente simétricos possuem freqüência temporal nula, estes modos podem ser pensados como parte do estado básico do modelo de equações aqui utilizado. De fato, conforme mostrado por Phillips (1966) num problema de ondas de gravidade internas, um campo de base com um cisalhamento fraco participa nas interações triádicas ressonantes "como se fosse" um modo de freqüência nula. O mesmo ocorre para uma topografia suave (Majda et al. 1999).
} 


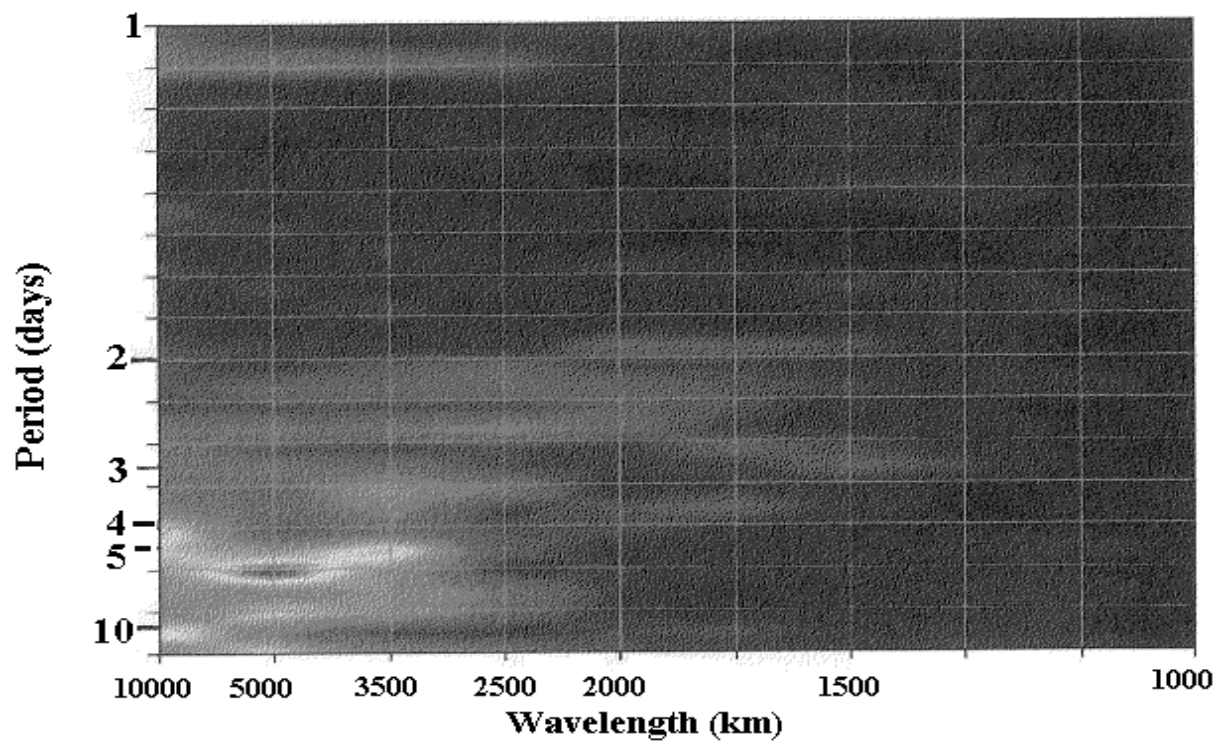

Figura 5.5: Espectro de potência da componente meridional do vento no equador para o período de 30 dias durante o experimento ABLE 2-b, usando dados da reanálise do NCEPNCAR. O eixo da abscissa representa o comprimento de onda de $10.000 \mathrm{Km}$ a $1.000 \mathrm{Km}$ e o eixo das ordenadas refere-se ao período das ondas (em dias). A análise espectral foi realizada no setor longitudinal de $90^{\circ} \mathrm{W}$ a $0^{\circ}$. (Fonte: Santos et al. 2002)

A variabilidade intra-sazonal da circulação atmosférica é caracterizada pela oscilação de Madden-Julian (OMJ) como sendo o modo de variabilidade dominante na região tropical, com um período característico da ordem de 30-60 dias. Além desse modo de variabilidade tropical, estudos observacionais também revelam a existência de padrões de teleconexões barotrópicos equivalentes ligando regiões tropicais e extratropicais que apresentam uma forte variabilidade na escala de tempo intra-sazonal (Wallace e Gutzler 1981; Ghil e Mo 1991; Magaña e Yanai 1991; Higgins e Mo 1997, e outros). Com isso, dado que os resultados apresentados nas Figuras 5.1 a 5.4 sugerem uma possível implicação das trocas de energia entre as ondas constituintes das tríades ressonantes no modelo atmosférico aqui adotado para a variabilidade intra-sazonal da circulação atmosférica, torna-se conveniente e bastante oportuno analisar as trocas de energia entre as ondas constituintes da tríade 6 da Tabela 4.5 devido à possível relação dos modos constituintes dessa tríade com alguns aspectos observados na circulação atmosférica de grande-escala na escala de tempo intra-sazonal. Por exemplo, a onda de Kelvin com número de onda zonal 1 e com a com a estrutura vertical do primeiro modo baroclínico apresenta várias características em comum com a oscilação de Madden-Julian (OMJ). De fato, a parte equatorial da oscilação de Madden-Julian (OMJ) 
apresenta uma estrutura global do tipo onda de Kelvin com número de onda zonal 1 e uma estrutura vertical essencialmente dada pelo primeiro modo baroclínico $\mathrm{m}=1$ mostrada na Figura $2.2 \mathrm{~b}$, possuindo uma velocidade de propagação para leste da ordem de $5 / \mathrm{ms}$ em regiões convectivamente ativas, como nas regiões do Índico Leste e do Pacífico Oeste, e da ordem de $15 \mathrm{~m} / \mathrm{s}$ fora dessas regiões fortemente convectivas (ver, por exemplo, Madden e Julian 1972; Madden e Julian 1994; Hendon e Salby 1994; Wheeler e Hendon 2004). As anomalias de Radiação de Onda Longa Emergente (ROLE) associadas a esta oscilação apresentam tipicamente um sinal mais forte sobre o Pacífico Equatorial Oeste e sobre o Indico Leste, apresentando uma oposição de fase do sinal entre essas duas regiões, e praticamente desaparecem à medida que o distúrbio se propaga para o Pacífico Central/Leste. No entanto, as anomalias de vento e pressão persistem conforme a oscilação se propaga ao longo do globo inteiro e modulam as circulações de Monções (Wang et al. 2005), bem como a atividade e/ou a intensidade dos furacões por meio da modulação no cisalhamento vertical do vento (Maloney e Hartmann 1999). Com relação à onda mista de Rossby-gravidade com número de onda zonal 2 constituinte da tríade 6 da Tabela 4.5, uma importante evidência observacional da atividade dessa onda na atmosfera tropical foi obtida por Wheeler e Kildadis (1999) através da análise espectral de uma série temporal de dados de ROLE. Wheeler e Kiladis (1999), ao comparar o espectro de potência obtido com as curvas de dispersão das ondas equatoriais para uma altura equivalente de $25 \mathrm{~m}$, verificaram um forte sinal, dentre outras ondas, das ondas de Yanai com números de onda 0-5. A concordância do pico espectral da ROLE nessa banda do espectro com a curva de dispersão das ondas mistas de Rossbygravidade para uma altura equivalente efetiva de $25 \mathrm{~m}$ (que é uma ordem de grandeza menor que a altura equivalente de aproximadamente $160 \mathrm{~m}$ associada ao primeiro modo baroclínico, de acordo com a Tabela 2.1) revela o forte acoplamento dessas ondas com a convecção nos trópicos ${ }^{6}$. Recompondo espectralmente o campo de ROLE na banda espectral das ondas de Yanai com números de onda 0-5, Wheeler e Kiladis também mostram que a atividade convectiva resultante dessas ondas é mais forte na região do Pacífico Central, próxima à linha de data.

Assim, dada a possível relação dos modos constituintes da tríade 6 da Tabela 4.5 com alguns aspectos observados acerca da circulação atmosférica de baixa freqüência e o

\footnotetext{
${ }^{6}$ No caso da incorporação dos efeitos diabáticos na equação da termodinâmica dada por (2.1e), supondo que o aquecimento resultante da liberação de calor latente seja proporcional ao movimento vertical, a inclusão desse efeito na equação da termodinâmica resultaria numa redução efetiva do parâmetro de estabilidade estática, o que por sua vez resultaria numa redução da constante de separação c nas equações (2.30) e, conseqüentemente, numa redução da velocidade de propagação das ondas lineares.
} 
significativo acoplamento entre esses modos mostrado na Tabela 4.5, a Figura 5.6 mostra a evolução temporal da energia modal para essa tríade, que é constituída por uma onda mista de Rossby-gravidade com número de onda zonal $2(\mathrm{k} \approx 0,314)$ e com a estrutura vertical do primeiro modo baroclínico $\mathrm{m}=1$ (modo a), uma onda de Kelvin com número de onda zonal 1 $(\mathrm{k} \approx 0,157)$ e com a estrutura vertical associada ao primeiro modo baroclínico $\mathrm{m}=1$ (modo $\mathbf{b})$ e uma onda de Rossby barotrópica com número de onda zonal $3(\mathrm{k} \approx 0,471)$ e com modo meridional $\mathrm{n}=2$ (modo $\mathbf{c})$.

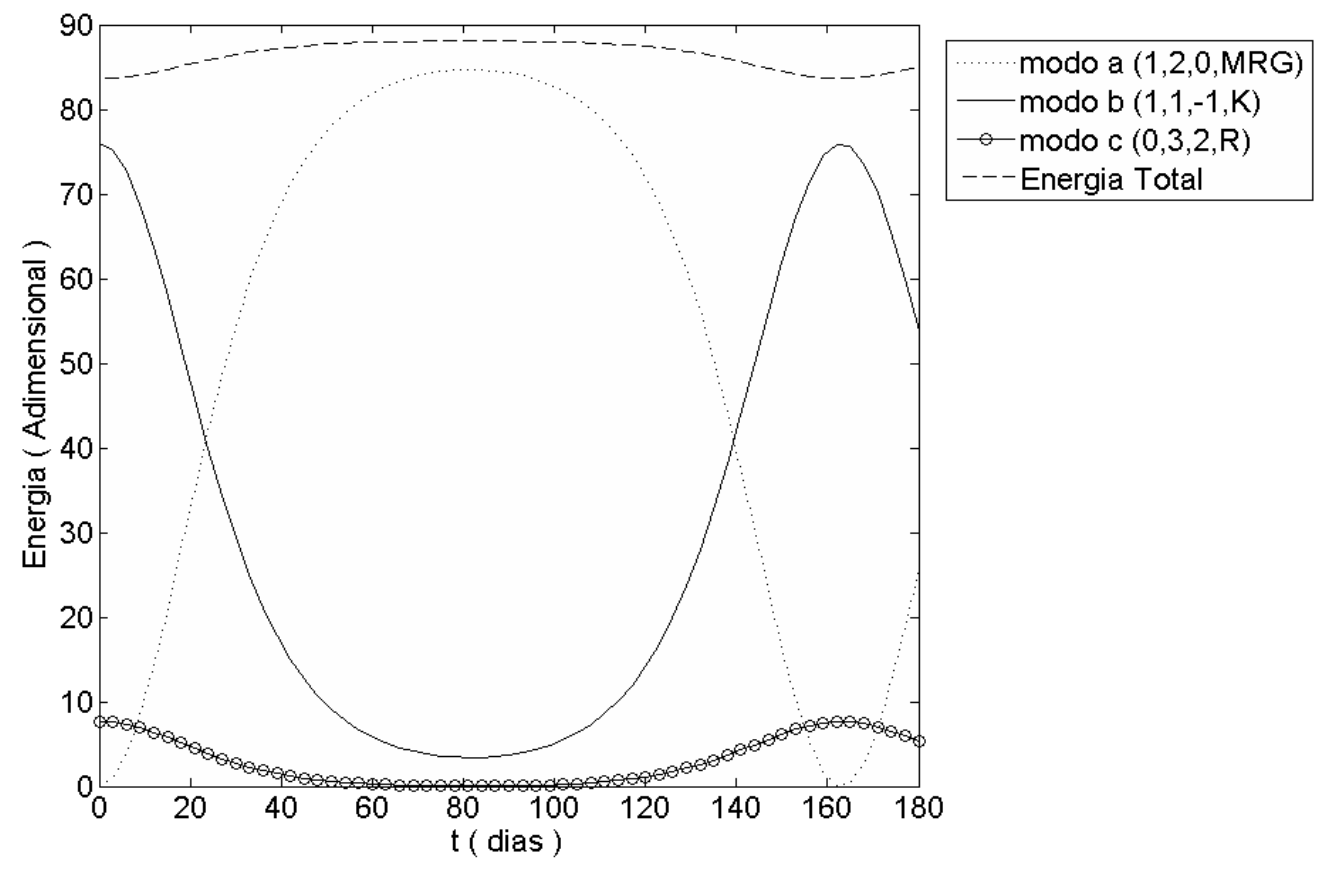

(a) 


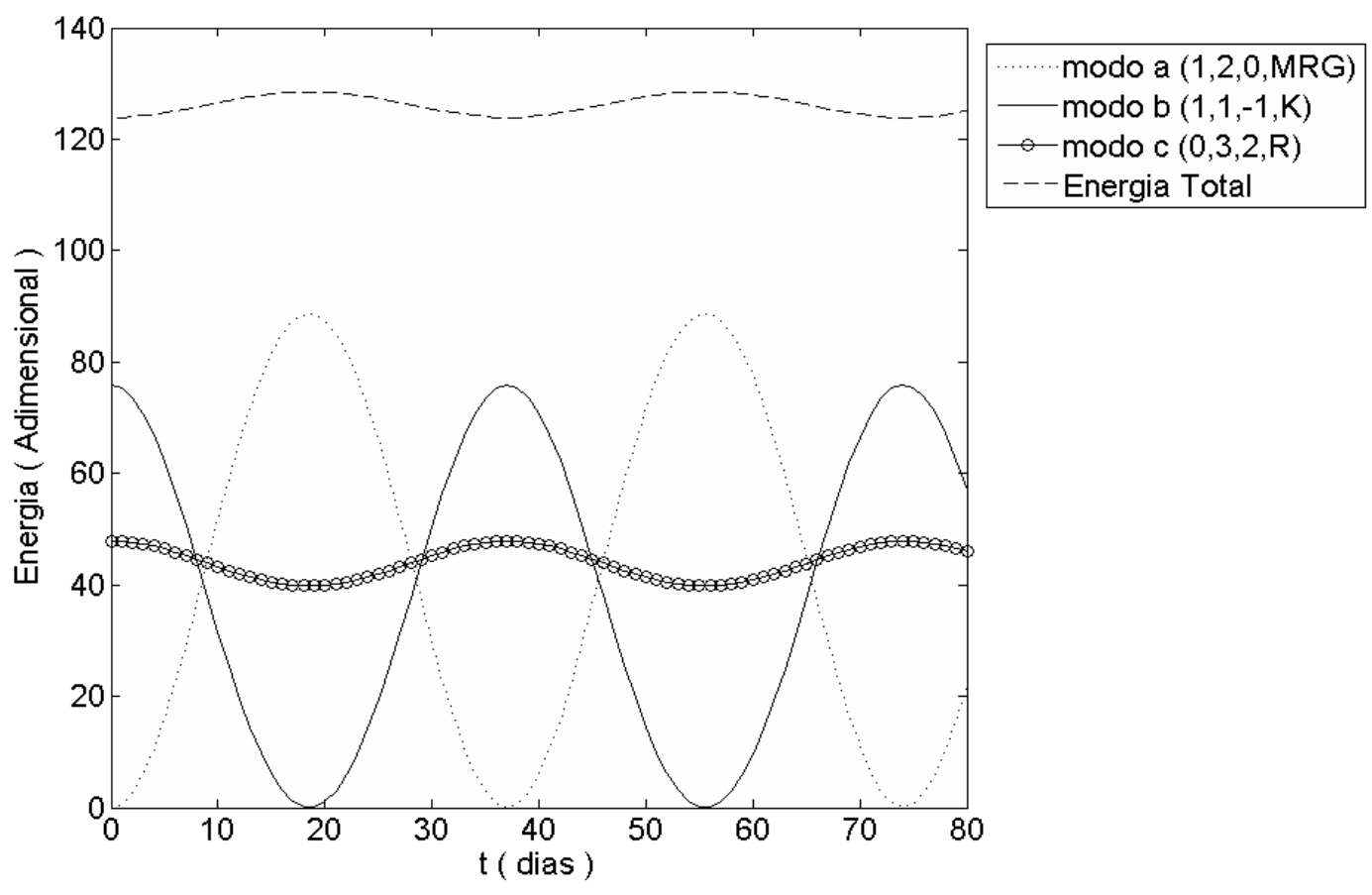

(b)

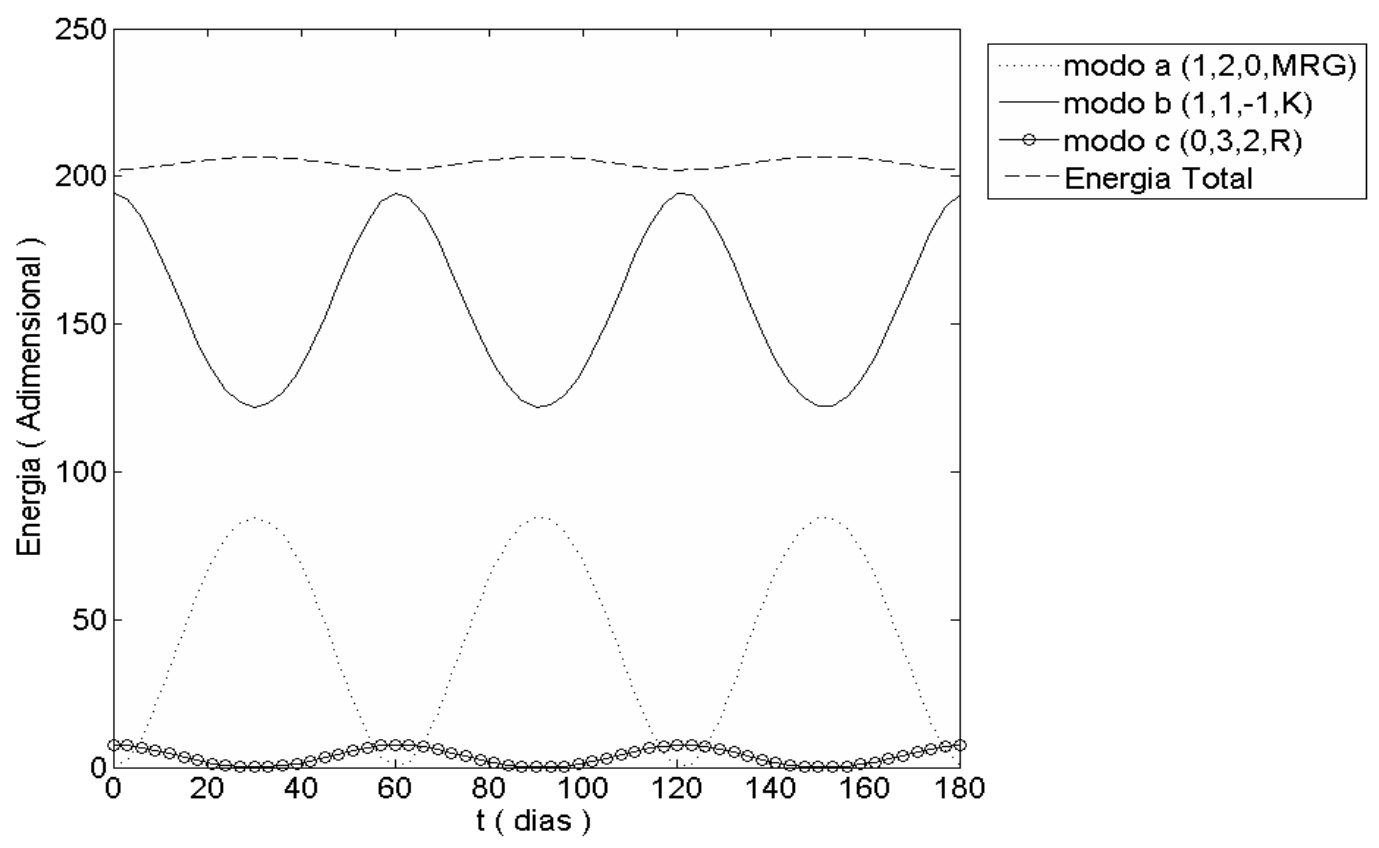

(c)

Figura 5.6: Evolução temporal da energia modal para a tríade constituída por: uma onda mista de Rossby-gravidade com número de onda zonal $2(\mathrm{k} \approx 0,314)$ e com a estrutura vertical do primeiro modo baroclínico $\mathrm{m}=1$ (modo a), uma onda de Kelvin com número de onda zonal 1 $(\mathrm{k} \approx 0,157)$ e com a estrutura vertical associada ao primeiro modo baroclínico $\mathrm{m}=1$ (modo b) e uma onda de Rossby barotrópica com número de onda zonal $3(\mathrm{k} \approx 0,471)$ e com modo 
meridional $\mathrm{n}=2$ (modo c). A figura mostra a evolução temporal da energia das ondas para a condição inicial caracterizada por: (a) $\mathrm{R}_{\mathrm{b}}{ }^{0}=5$ e $\mathrm{R}_{\mathrm{c}}{ }^{0}=0,2$; (b) $\mathrm{R}_{\mathrm{b}}{ }^{0}=5$ e $\mathrm{R}_{\mathrm{c}}{ }^{0}=0,5$ e (c) $\mathrm{R}_{\mathrm{b}}{ }^{0}=8$ e $\mathrm{R}_{\mathrm{c}}{ }^{0}=0,2$.

Através da Figura 5.6 observa-se que, como esperado a partir dos valores dos coeficientes de interação para a tríade 6 da Tabela 4.5, a interação entre os modos constituintes desse tripleto é caracterizada pela onda mista de Rossby-gravidade como sendo uma fonte ou sumidouro de energia para as ondas de Kelvin e de Rossby barotrópica, ou seja, a onda mista sempre cede energia para as demais ou recebe energia dos demais modos constituintes desse tripleto ressonante. Neste caso, a variação da energia da onda de Rossby barotrópica é bem menor em comparação com as variações de energia das ondas de Kelvin e de Yanai baroclínicas, como verificado na Figura 5.6. Assim como observado na interação referente à Figura 5.1, é possível notar na Figura 5.6 algumas pequenas oscilações na energia total dessa tríade ressonante, que são devidas ao fato de que a condição de ressonância para as freqüências temporais não é exatamente satisfeita em razão da natureza discreta do espectro, como discutido anteriormente. No entanto, observa-se que essa variação da energia total é menor que a variação da energia da onda de Rossby, o que mostra que a ressonância para esse tripleto é bastante robusta e bem próxima da ressonância exata. Além disso, embora o modo b dessa tríade ressonante esteja inserido num "pacote" de ondas não dispersivo (Figura 4.4), as auto-interações envolvendo as componentes desse pacote são pequenas, pois essas componentes do modo de Kelvin estão associadas ao mesmo modo interno. Logo, além da ressonância envolvendo os modos da tríade 6 da Tabela 4.5 ser robusta, acredita-se que a interação entre os modos desse tripleto seja, de fato, relevante para a dinâmica das equações primitivas completas.

$\mathrm{Na}$ integração mostrada na Figura 5.6a, na qual a condição inicial é dada por $\mathrm{R}_{\mathrm{b}}{ }^{0}=5 \mathrm{e}$ $\mathrm{R}_{\mathrm{c}}{ }^{0}=0,2$, a onda de Yanai, cuja energia é nula no instante inicial, é rapidamente excitada, retirando energia das ondas de Rossby e de Kelvin, e atinge o mesmo nível energético do modo de Kelvin em $t \approx 25$ dias. A onda de Yanai segue então retirando energia das ondas de Rossby e de Kelvin até aproximadamente $\mathrm{t}=80$ dias, passando então a agir como uma fonte de energia para esses modos até aproximadamente 160 dias de integração, quando o ciclo então se repete. É interessante notar que entre $t=70$ e 90 dias, que corresponde ao período no qual o modo de Yanai atinge seu patamar energético, a onda de Rossby externa torna-se energeticamente nula. Na integração ilustrada na Figura 5.6b, a condição inicial é dada por 
$\mathrm{R}_{\mathrm{b}}{ }^{0}=5$ e $\mathrm{R}_{\mathrm{c}}{ }^{0}=0,5$. Neste caso, observa-se o mesmo ciclo energético observado na Figura 5.6a, mas com uma escala de tempo bem mais curta, apresentando um período da ordem de 40 dias. Nota-se ao comparar as Figuras 5.6a e 5.6b que, aumentando a amplitude inicial do modo de Rossby por um fator de 2,5, o período das trocas de energia entre os modos constituintes dessa tríade reduz de 160 dias para cerca de 40 dias. Logo, verifica-se ao comparar as Figuras 5.6a e 5.6b que o período das trocas de energia entre os modos constituintes de uma tríade ressonante no modelo atmosférico aqui adotado pode ser arbitrariamente longo ou arbitrariamente curto, dependendo da amplitude inicial do modo menos energeticamente ativo do tripleto, que neste caso corresponde à onda de Rossby barotrópica com número de onda 3 e modo meridional $n=2$. Na integração referente à Figura 5.6c, a condição inicial é dada por $\mathrm{R}_{\mathrm{b}}{ }^{0}=8$ e $\mathrm{R}_{\mathrm{c}}{ }^{0}=0,2$. Assim, comparando as soluções dispostas nas Figuras 5.6a e 5.6c nota-se que, mantendo-se a amplitude inicial do modo de Rossby e aumentando-se a amplitude inicial do modo de Kelvin, é possível observar que o período das trocas de energia entre os modos envolvidos nessa tríade também reduz consideravelmente, tornando-se aproximadamente igual a 60 dias. Entretanto, também é possível observar na Figura 5.6c que, neste caso, a troca de energia é menos expressiva que as observadas nas Figuras 5.6a e 5.6b, i.e, a solução disposta na Figura 5.6c torna-se mais estável em comparação com os casos anteriores. Esta maior estabilidade da solução referente à Figura 5.6c deve-se ao fato de que, ao considerar a amplitude inicial da onda de Kelvin muito maior que as amplitudes das ondas de Rossby e de Yanai, como é o caso da integração correspondente à Figura 5.6c, a análise de estabilidade dada pelas equações (5.6) mostra que, neste caso, as energias das ondas de Rossby e de Yanai continuam pequenas em comparação com a energia da onda de Kelvin, uma vez que os coeficientes de interação dos modos de Rossby e de Yanai possuem sinais opostos. Conseqüentemente, quando a amplitude inicial da onda de Kelvin é muito maior que as amplitudes iniciais das ondas de Rossby e de Yanai, a troca de energia nesta tríade é fortemente inibida. O mesmo ocorre quando a amplitude inicial da onda de Rossby é muito maior que a amplitude dos demais modos dessa tríade (figura não mostrada). Se um desses três modos constituintes da tríade 6 da Tabela 4.5 tiver a amplitude inicial muito maior que a dos demais modos constituintes dessa tríade, as amplitudes dos demais modos somente crescerão com o tempo e, conseqüentemente, somente ocorrerá uma significativa troca de energia, se esse modo em particular corresponder à onda de Yanai, uma vez que a onda de Yanai é a onda de maior freqüência absoluta e, portanto, com o coeficiente de interação com o sinal oposto ao dos demais na tríade referente à Figura 5.6. 
Dessa forma, os resultados dispostos na Figura 5.6 mostram que a escala de tempo e a magnitude das trocas de energia entre as ondas num dado tripleto ressonante dependem da energia inicial e da maneira como a energia inicial é distribuída entre as componentes do tripleto ressonante. Os resultados destacam que o período das trocas de energia para a tríade ressonante mostrada na Figura 5.6 pode ser arbitrariamente longo ou arbitrariamente curto quanto menor ou maior, respectivamente, for as amplitudes iniciais dos modos de Rossby barotrópico e de Kelvin baroclínico. Para as condições iniciais consideradas nas integrações referentes às Figuras 5.6b e 5.6c, as trocas de energia entre os modos dessa tríade ocorrem na escala de tempo intra-sazonal, com um período de aproximadamente 40 e 60 dias, respectivamente. Já para a condição inicial considerada na integração referente à Figura 5.6a, as trocas de energia apresentam uma periodicidade semi-anual. Os resultados também ilustram que à medida que a amplitude inicial da onda de Kelvin torna-se maior que as amplitudes iniciais das ondas de Rossby e de Yanai, a solução torna-se mais estável, ou seja, há uma inibição das trocas de energia entre os modos envolvidos nessa tríade.

Com o intuito de ilustrar as implicações das trocas de energia mostradas na Figura 5.6 para a solução de ordem dominante no espaço físico, as Figuras 5.7 a 5.9 destacam alguns aspectos da solução do problema de três ondas ressonantes (5.8) para a tríade referente à Figura 5.6 no espaço físico. Neste caso, os campos do vento e do geopotencial correspondentes à solução de ordem dominante do modelo aqui adotado são obtidos a partir das amplitudes $R_{\mathrm{j}}(\mathrm{t}), \mathrm{j}=\mathbf{a}, \mathbf{b}$ e c, através da expansão espectral representada por (2.53) truncada de modo a considerar somente os três modos da tríade ressonante explorada na Figura 5.6. A Figura 5.7 ilustra a distribuição horizontal dos campos do vento horizontal e do geopotencial em $1000 \mathrm{hPa}$, associados à mesma solução mostrada na Figura 5.6a, em $\mathrm{t}=0$ (Figura 5.7a), em $\mathrm{t}=25$ dias (Figura 5.7b) e em $\mathrm{t}=80$ dias (Figura 5.7c). Em t $=0$, a estrutura espacial dos campos do vento horizontal e do geopotencial dispostos na Figura 5.7a é devida à superposição das atividades da onda de Kelvin e de Rossby externa, uma vez que a energia da onda mista de Rossby-gravidade é nula neste instante inicial. De fato, nota-se claramente na Figura 5.7a uma forte componente zonal do vento em fase com o geopotencial ao longo do equador e com uma estrutura ondulatória com número de onda zonal 1. Esta estrutura do vento zonal em fase com o geopotencial confinada equatorialmente é resultante da atividade da onda de Kelvin. Como discutido anteriormente, este modo de Kelvin com número de onda 1 e com a estrutura vertical correspondente ao primeiro modo baroclínico exibe vários aspectos em comum com a oscilação de Madden-Julian nos trópicos. Inclusive, na fase oposta ao padrão ilustrado na Figura 5.7a da onda de Kelvin o vento zonal de oeste sobre a região do 
Pacífico Oeste decorrente da propagação para leste desse modo, devido ao acoplamento da atmosfera real com o oceano, pode ter um significativo impacto na temperatura da superfície do mar, nos fluxos de calor na superfície e na estrutura da Termoclina oceânica (ver, por exemplo, Zhang 1996; Jones e Weare 1996; Jones et al. 1998; Kessler et al. 1996). Este mecanismo, conhecido como "explosão de vento de oeste", tem sido inclusive sugerido como um possível mecanismo no disparo dos eventos quentes de ENSO (El Niño-Oscilação Sul) (Lau e Chan 1986; Weickmann 1991). A pequena componente meridional do vento ao longo do equador observada na Figura 5.7a, que apresenta uma estrutura ondulatória com número de onda 3, é resultante da atividade da onda de Rossby externa.

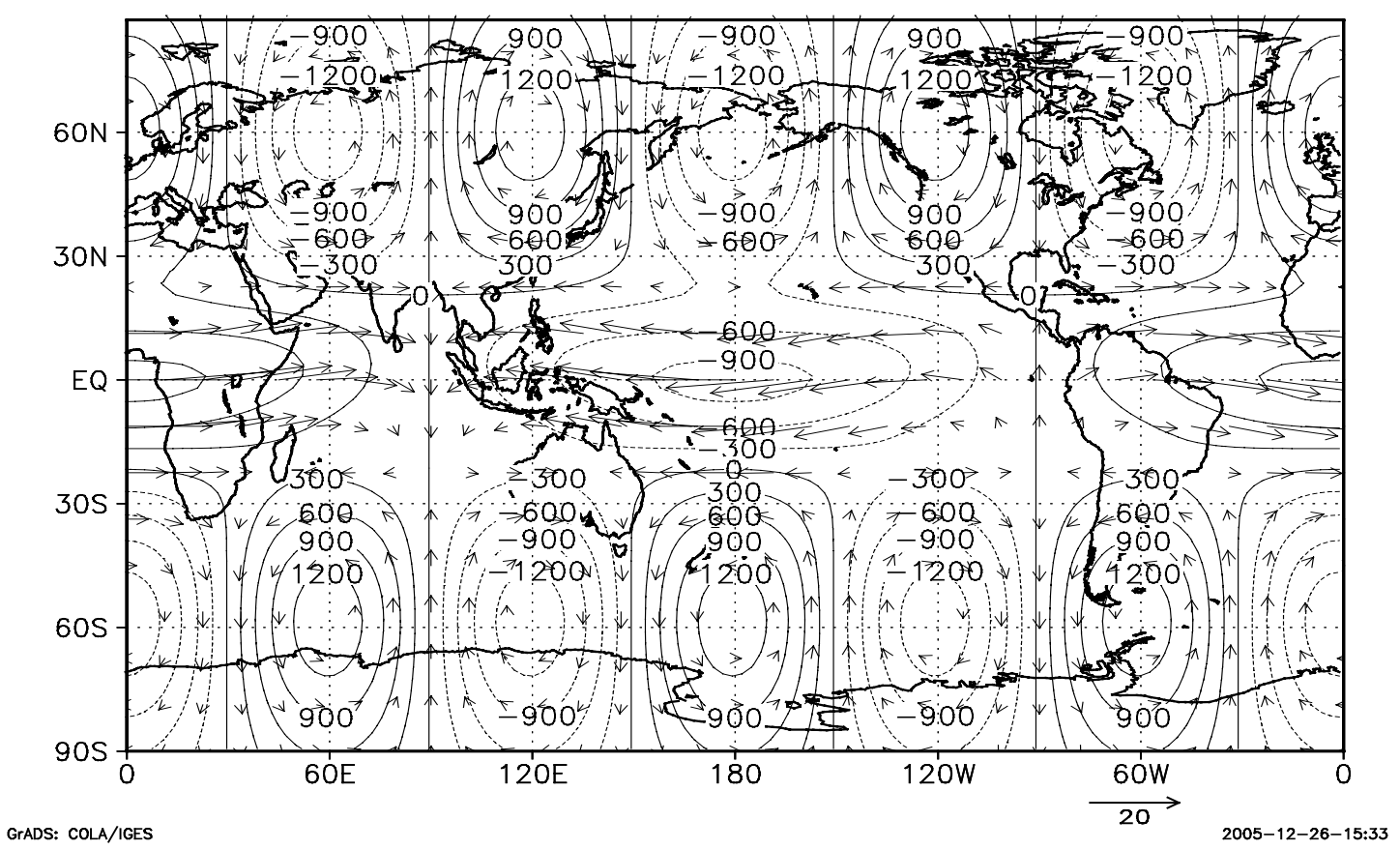

(a) 


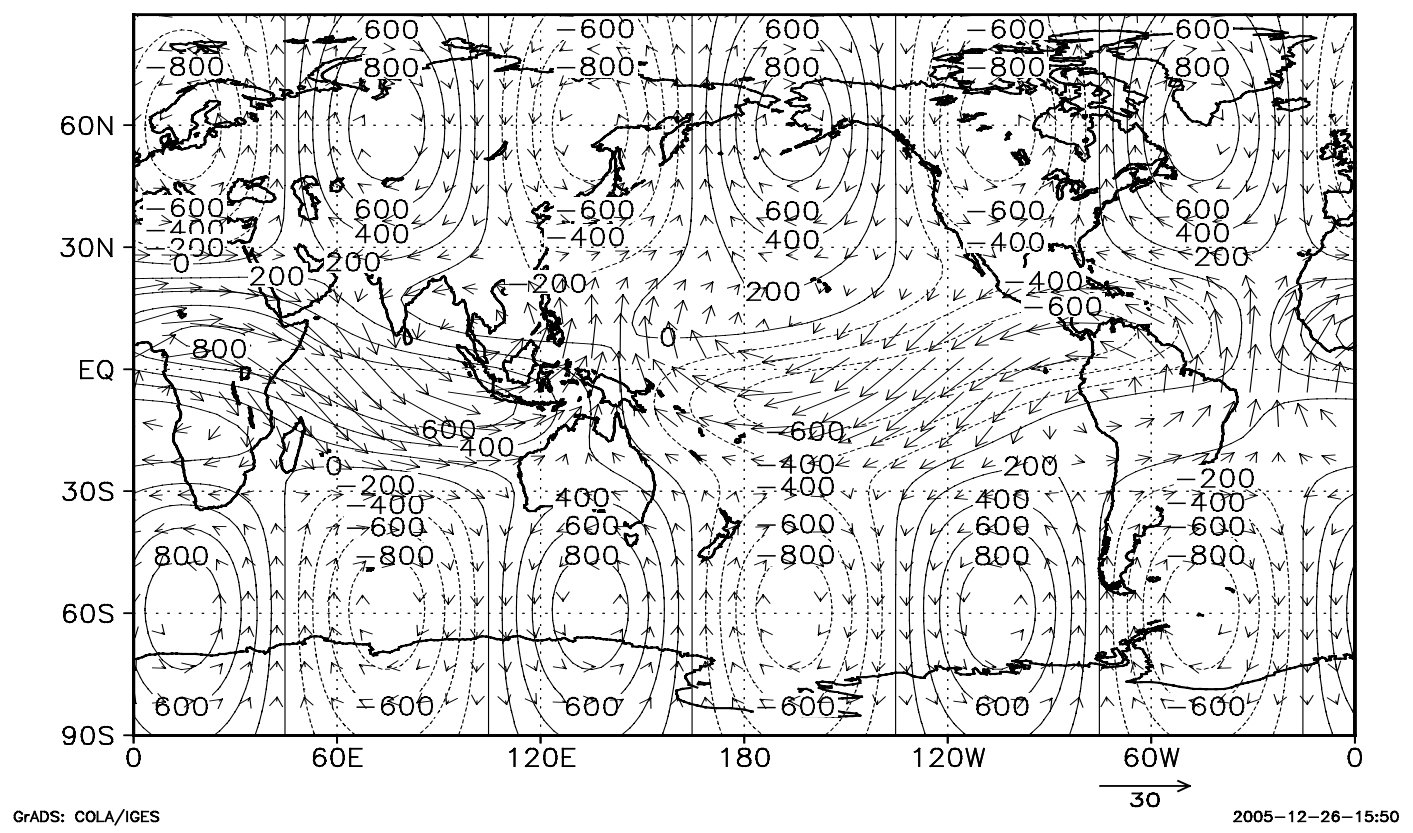

(b)

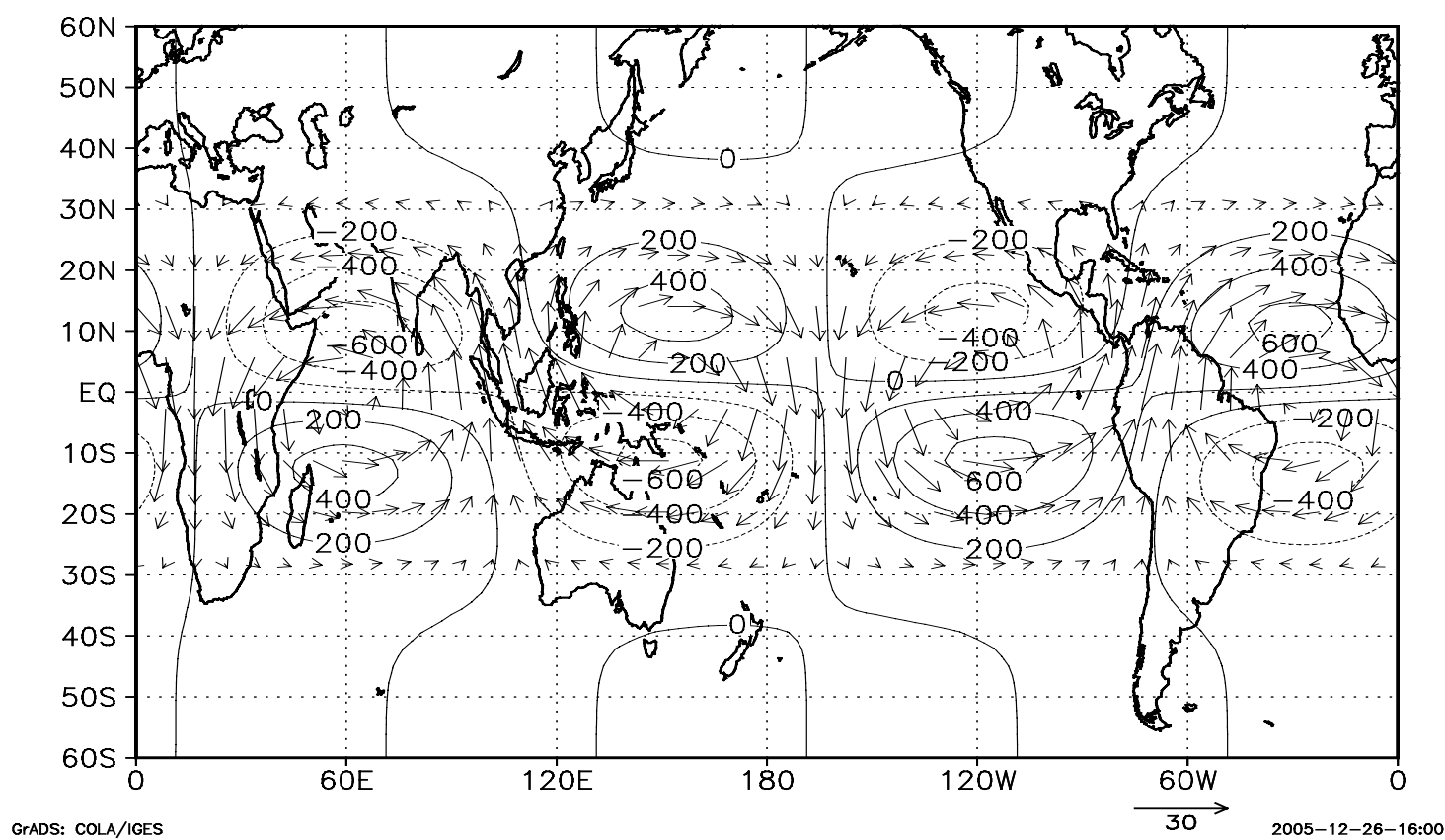

(c)

Figura 5.7: Distribuição horizontal dos campos do vento horizontal (vetor) e do geopotencial (contornos) em $\mathrm{p}=1000 \mathrm{hPa}$ associados à mesma solução mostrada na Figura 5.6a em: (a) $\mathrm{t}=$ 0 ; (b) $t=25$ dias e (c) $t=80$ dias. Os campos do vento horizontal e do geopotencial são dados, respectivamente, em $\mathrm{m} / \mathrm{s} \mathrm{e} \mathrm{em} \mathrm{m}^{2} \mathrm{~s}^{-2}$, usando as escalas dadas por (2.11) para $\mathrm{U}=$ $5 \mathrm{~m} / \mathrm{s}, \beta=2,3 \times 10^{-11} \mathrm{~m}^{-1} \mathrm{~s}^{-1} \mathrm{e} \mathrm{L}=10^{6} \mathrm{~m}$. 
A seqüência alternada de vórtices ciclônicos e anticiclônicos centrados em $60^{\circ}$ de latitude (Sul e Norte) apresentando uma estrutura ondulatória com número de onda 3, com um notável balanço geostrófico entre os campos do vento e do geopotencial, são também resultantes da atividade da onda de Rossby barotrópica. A estrutura espacial global da onda de Rossby barotrópica constituinte da tríade 6 da Tabela 4.5 é mais claramente notável na Figura 5.8, que mostra a distribuição horizontal dos campos do vento horizontal e do geopotencial em $\mathrm{t}=0$ referentes à mesma solução das Figuras 5.6a e 5.7, mas no nível de 500hPa. Como discutido anteriormente, os campos dispostos neste nível de pressão ilustram essencialmente a atividade das ondas associadas ao modo barotrópico, uma vez que a autofunção $G_{1}(p)$ da equação da estrutura vertical do problema de $\mathrm{O}(1)$ é aproximadamente nula neste nível. Notase na Figura 5.8 a mesma seqüência de vórtices ciclônicos e anticiclônicos centrados em $60^{\circ}$ de latitude observada em $p=1000 \mathrm{hPa}$ e exatamente em fase com seqüência de vórtices observada na baixa troposfera, o que evidencia a estrutura barotrópica desses centros de ação. Um outro aspecto interessante a ser observado acerca da estrutura horizontal da onda de Rossby barotrópica na Figura 5.8 é a oposição de fase entre a vorticidade na região tropical e em latitudes médias e altas.

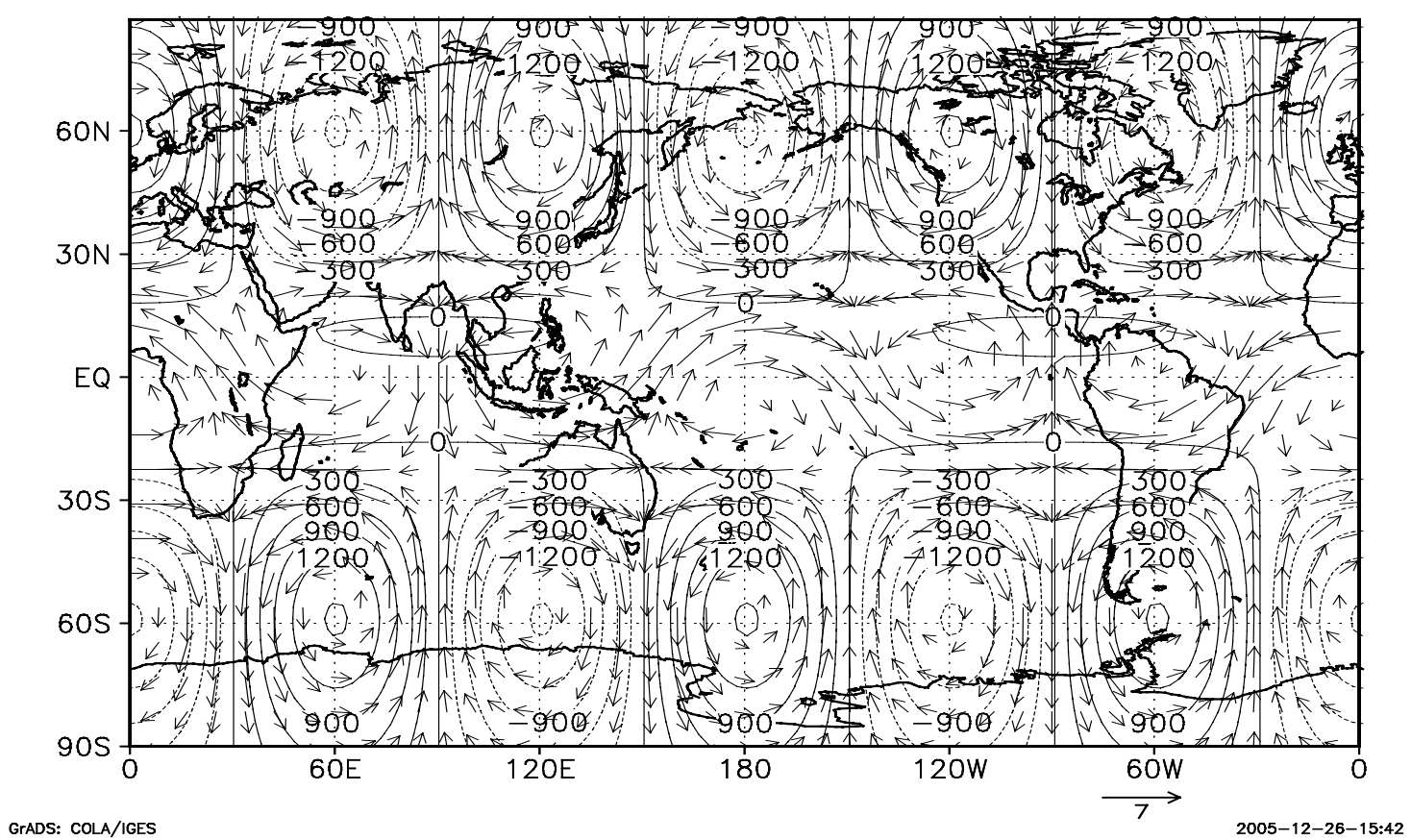

Figura 5.8: Análoga à Figura 5.7a, mas com as variáveis dispostas no nível $\mathrm{p}=500 \mathrm{hPa}$. 
Diversos trabalhos (Ghil e Mo 1991; Rogers e van Loon 1982; Kidson 1988, 1991; Mo e Higgins 1998), utilizando dados médios mensais ou dados diários ou pentadais filtrados na escala de baixa freqüência obtidos de reanálises de modelos operacionais de previsão de tempo, fizeram análise de Componentes Principais para os dados no Hemisfério Sul e mostram que as duas primeiras Funções Ortogonais Empíricas (EOFs) para a função de corrente são caracterizadas por padrões ondulatórios com número de onda zonal $3 \mathrm{em}$ médias latitudes, com uma maior amplitude sobre o setor do Pacífico e da América do Sul, apresentando uma aproximada oposição de fase entre a circulação em médias latitudes e nos sub-trópicos. Estes dois primeiros padrões espaciais obtidos nesses estudos estão em quadratura de fase um com o outro e, devido à semelhança desses padrões com o padrão do Pacífico/América do Norte (PNA) verificado inicialmente por Wallace e Gutzler (1981), são comumente rotulados como padrões do Pacífico/América do Sul (PSA-1 e PSA-2). Estes estudos também mostram através da evolução das componentes principais associadas a estes modos que estes padrões do PSA são modulados principalmente na escala de tempo interanual e intra-sazonal. Mo e Higgins (1998) mostram que os espectros das componentes principais associadas às duas primeiras EOFs apresentam um pico significativo na escala de 36-40 dias e, através de um composto da Radiação de Onda Longa Emergente (ROLE) para os períodos de intensa magnitude desses padrões do PSA, sugerem que estes padrões estejam intimamente relacionados com as anomalias convectivas resultantes da atividade da oscilação de Madden-Julian (OMJ) nos trópicos. Mo e White (1985) também obtiveram uma evidência observacional de padrões de teleconexões em médias latitudes, apresentando uma estrutura barotrópica equivalente, com estrutura ondulatória de número de onda zonal 3.

Logo, a estrutura espacial da onda de Rossby barotrópica ilustrada na Figura 5.8 apresenta vários aspectos em comum com os padrões do PNA e do PSA. A diferença é que a estrutura da onda de Rossby mostrada na Figura 5.8 é mais global, ao passo que as magnitudes dos padrões do PNA e do PSA são mais intensas sobre o setor do Pacífico e do continente Americano. Tal diferença pode ser atribuída à ausência de um estado básico mais realista no modelo atmosférico aqui adotado. Da mesma maneira, a curvatura para leste da trajetória do trem de ondas provindo dos trópicos em direção aos extratrópicos comumente observada nos padrões do PNA e do PSA (Karoly 1989; Horel e Wallace 1981; Mo e Higgins 1998) também não é observada na Figura 5.8 e essa ausência também pode ser atribuída à ausência de um estado básico mais realista no modelo atmosférico aqui adotado. De fato, diversos trabalhos (Hoskins et al. 1977; Hoskins e Karoly 1981; Hoskins e Ambrizzi 1993) 
mostram que na presença de um campo básico realista a trajetória da propagação da energia das ondas de Rossby barotrópicas geradas em latitudes tropicais sofre uma curvatura para leste em direção ao equador na região do jato polar em decorrência da refração dessas ondas devido ao intenso gradiente meridional de vorticidade absoluta nessas regiões.

Com isso, de acordo com os resultados aqui apresentados, o mecanismo de ressonância não linear acoplando a onda de Kelvin baroclínica com número de onda zonal 1 com a onda de Rossby barotrópica com número de onda zonal 3 e com modo meridional $n=$ 2 por meio da onda de Yanai baroclínica e com número de onda zonal 2 pode ser um possível mecanismo de acoplamento entre a OMJ nos trópicos e os padrões de teleconexões trópicosextratrópicos do PNA e do PSA.

Em $\mathrm{t}=25$ dias, a onda mista de Rossby-gravidade e a onda de Kelvin possuem praticamente o mesmo nível energético (Fig. 5.6a). Como resultado, a Figura 5.7b mostra que nesse instante de tempo o escoamento atmosférico na região tropical é resultante da superposição das atividades desses dois modos. É notável na Figura $5.7 \mathrm{~b}$ a contribuição da onda mista de Rossby-gravidade através de uma razoável componente meridional do vento ao longo do equador apresentando uma estrutura ondulatória com número de onda 2 . Também é possível observar na Figura $5.7 \mathrm{~b}$ que os vórtices quase-geostróficos centrados em $60^{\circ}$ de latitude resultantes da atividade da onda de Rossby barotrópica apresentam uma notável diminuição de magnitude em comparação com o instante inicial, em decorrência do decréscimo da energia da onda de Rossby. Em $t=80$ dias, o escoamento é essencialmente resultante da atividade da onda mista de Rossby-gravidade (Figura 5.7c), dado o amplo predomínio energético desse modo nesse instante de tempo (Figura 5.6a).

A Figura 5.9 ilustra a evolução temporal da componente zonal do vento no nível de $1000 \mathrm{hPa}$ em $\left(180^{\circ}, 0^{\circ}\right)$ (Figura 5.9a), da componente zonal do vento no nível de $500 \mathrm{hPa}$ em $\left(180^{\circ}, 25^{\circ} \mathrm{S}\right)$ (Figura $\left.5.9 \mathrm{~b}\right)$ e da componente meridional do vento no nível de $1000 \mathrm{hPa}$ em $\left(180^{\circ}, 0^{\circ}\right)$ (Figura 5.9c). 


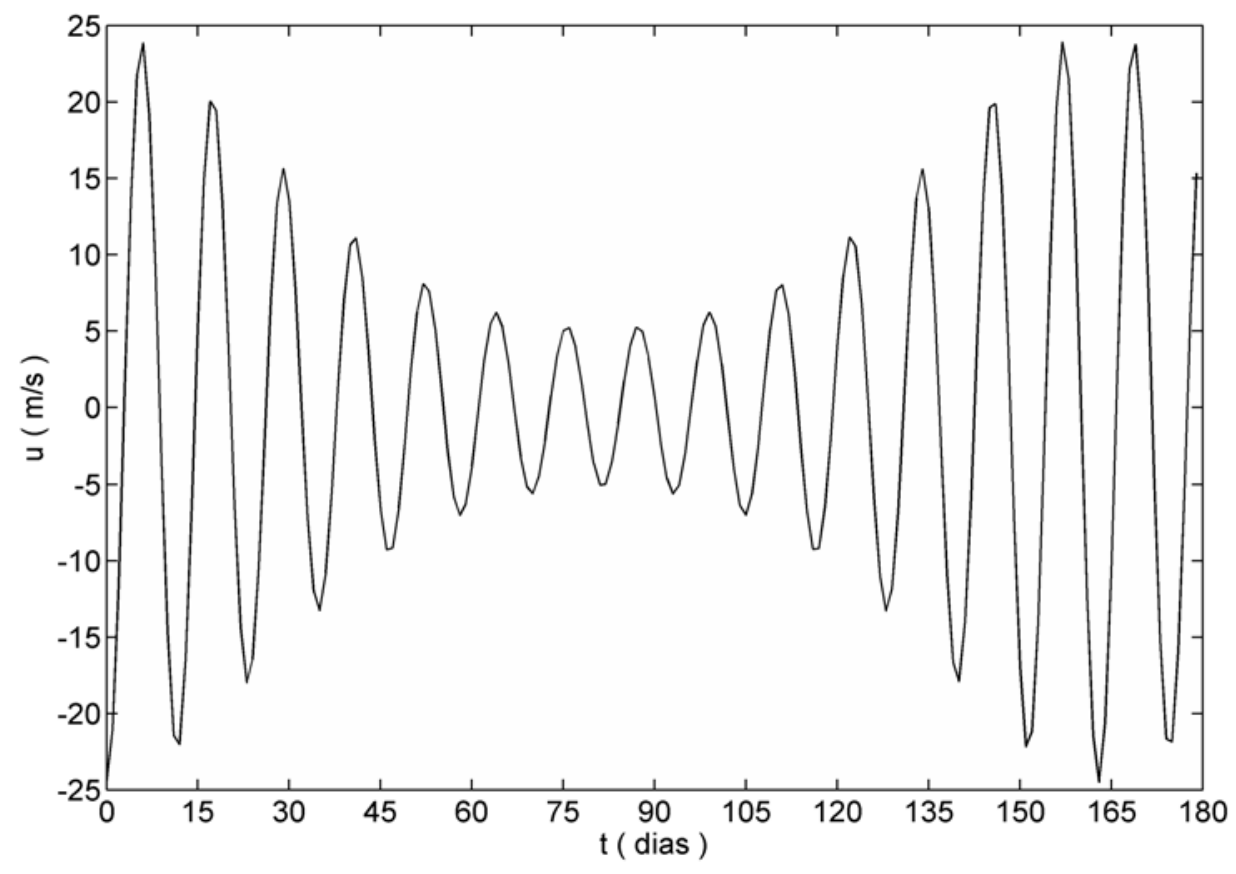

(a)

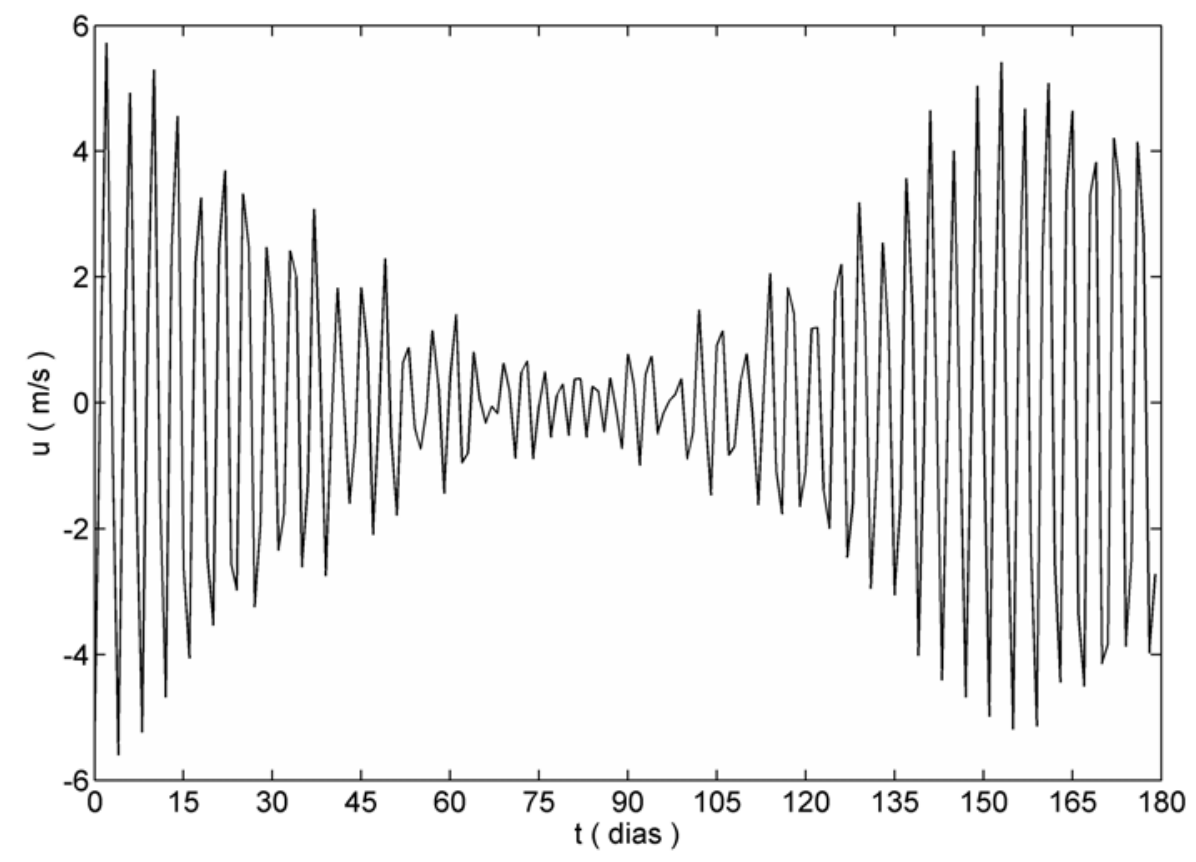

(b) 


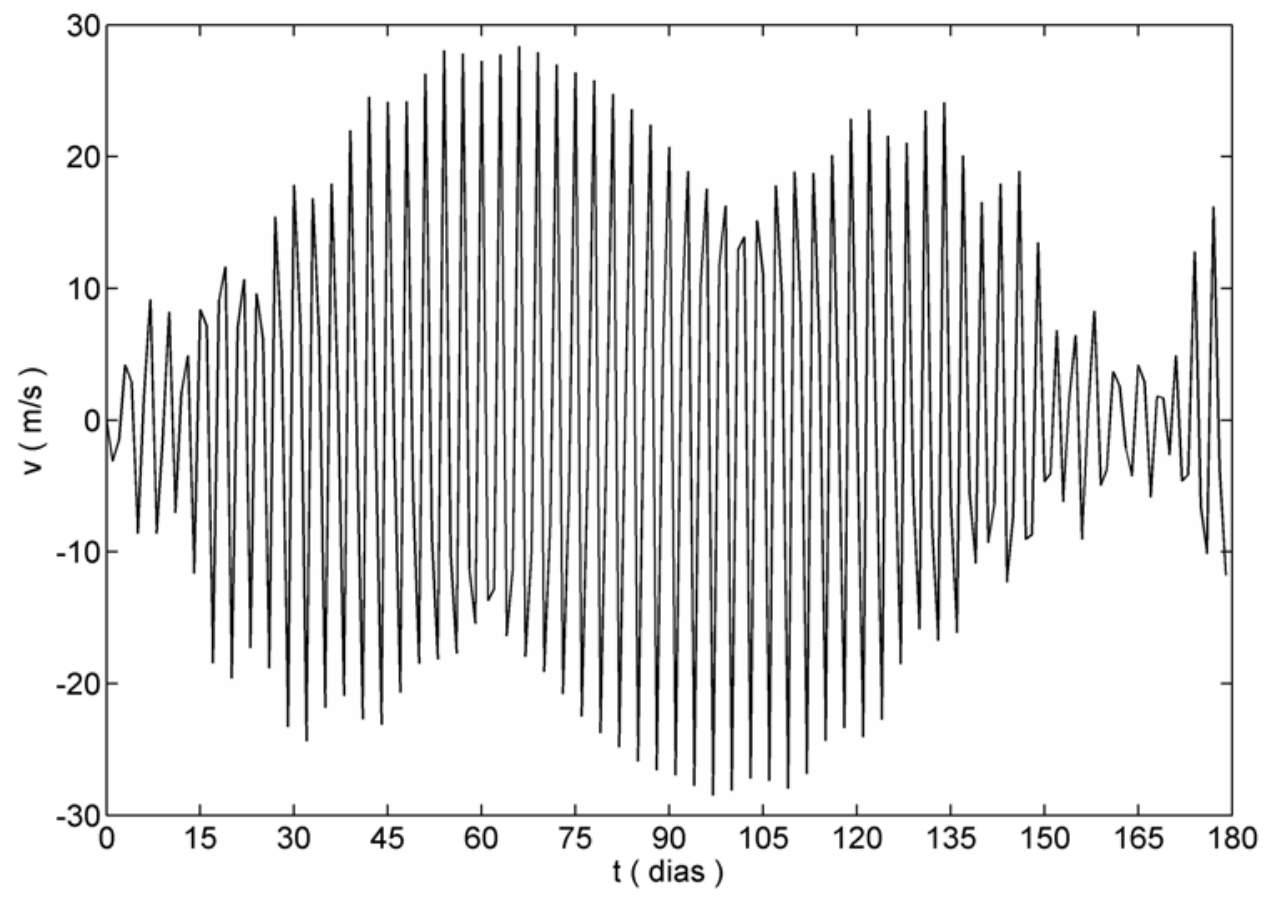

(c)

Figura 5.9: Evolução temporal da componente zonal do campo do vento no nível de $1000 \mathrm{hPa}$ em $\left(180^{\circ}, 0^{\circ}\right)$ (a); da componente zonal do campo do vento no nível de $500 \mathrm{hPa}$ em $\left(180^{\circ}, 25^{\circ} \mathrm{S}\right)$ (b) e da componente meridional do campo do vento no nível de $1000 \mathrm{hPa}$ em $\left(180^{\circ}, 0^{\circ}\right)(\mathrm{c})$. Os gráficos mostrados nessa figura correspondem à solução referente às Figuras 5.6a, 5.7 e 5.8. Os campos de u e v são dados em m/s, usando as escalas dadas por (2.11) para $\mathrm{U}=5 \mathrm{~m} / \mathrm{s}$.

A componente zonal do vento ao longo do equador é a melhor variável que caracteriza o sinal das perturbações decorrentes da atividade das ondas de Kelvin, enquanto, como discutido anteriormente, a componente meridional do vento ao longo do equador é o fator que melhor caracteriza a atividade das ondas mistas de Rossby-gravidade. Da mesma maneira, de acordo com a Figura 5.8, a componente zonal do campo do vento no nível de 500hPa, que está associada à atividade da onda de Rossby barotrópica, apresenta máximos exatamente ao longo da latitude de $\pm 25^{\circ}$. Conseqüentemente, as evoluções temporais das componentes do vento horizontal observadas nas Figuras 5.9a, 5.9b e 5.9c estão associadas às atividades dos modos de Kelvin, Rossby e Yanai, respectivamente, constituintes da tríade ressonante 6 da Tabela 4.5. Assim como na Figura 5.4, as oscilações locais de mais alta freqüência observadas 
na Figura 5.9 decorrem da própria propagação de fase dos modos, ao passo que a modulação de freqüência mais baixa nessas oscilações locais é resultante da modulação da amplitude/energia dos modos, i.e, resulta das trocas de energia entre os modos observadas na Figura 5.6a. De fato, comparando a Figura 5.9 com a Figura 5.6a é possível verificar que as modulações de período mais longo nas oscilações locais das componentes do campo do vento horizontal observadas nas Figuras 5.9a, 5.9b e 5.9c estão exatamente em fase com as oscilações na energia dos modos de Kelvin, Rossby e Yanai, respectivamente. Logo, as periódicas mudanças de regime na solução no espaço físico decorrentes das trocas de energia entre os modos constituintes desse tripleto ressonante são também observadas a partir das figuras 5.7 e 5.9. Os períodos das ondas de Kelvin, Rossby externa e Yanai observados através das oscilações locais nas Figuras 5.9a, 5.9b e 5.9c, respectivamente, são de 13 dias, 5 dias e 3 dias, respectivamente. O período da onda de Kelvin de aproximadamente 13 dias implica numa velocidade de propagação de aproximadamente $40 \mathrm{~m} / \mathrm{s}$. Esta velocidade de fase é um tanto irrealista quando comparada com as velocidades de propagação típicas da oscilação de Madden-Julian (OMJ) nos trópicos. Isto também pode ser atribuído à ausência no modelo atmosférico aqui adotado dos processos convectivos úmidos, ou seja, da interação entre as ondas de grande-escala e a convecção úmida. Como discutido anteriormente, a inclusão do termo de aquecimento diabático na equação da termodinâmica (2.1e) e a parametrização deste em termos do movimento vertical leva à redução efetiva do parâmetro de estabilidade estática e, conseqüentemente, à redução na velocidade de propagação das ondas lineares.

A Figura 5.10 ilustra a distribuição horizontal dos campos do vento horizontal e do geopotencial em $1000 \mathrm{hPa}$ no instante inicial $(\mathrm{t}=0)$ associados à solução referente à Figura 5.6b. Como pode ser observado na Figura 5.10, as amplitudes iniciais dos modos consideradas na integração referente à Figura $5.6 \mathrm{~b}$ também reproduzem magnitudes típicas das perturbações observadas no escoamento atmosférico de grande-escala. Logo, os resultados apresentados nas Figuras 5.6 a 5.10 destacam que, para as amplitudes iniciais dos modos caracterizando magnitudes típicas das perturbações observadas no escoamento atmosférico de grande-escala, os modos constituintes da tríade ressonante referente à Figura 5.6 trocam energia tanto na escala intra-sazonal quanto numa escala semi-anual, dependendo da condição inicial. 


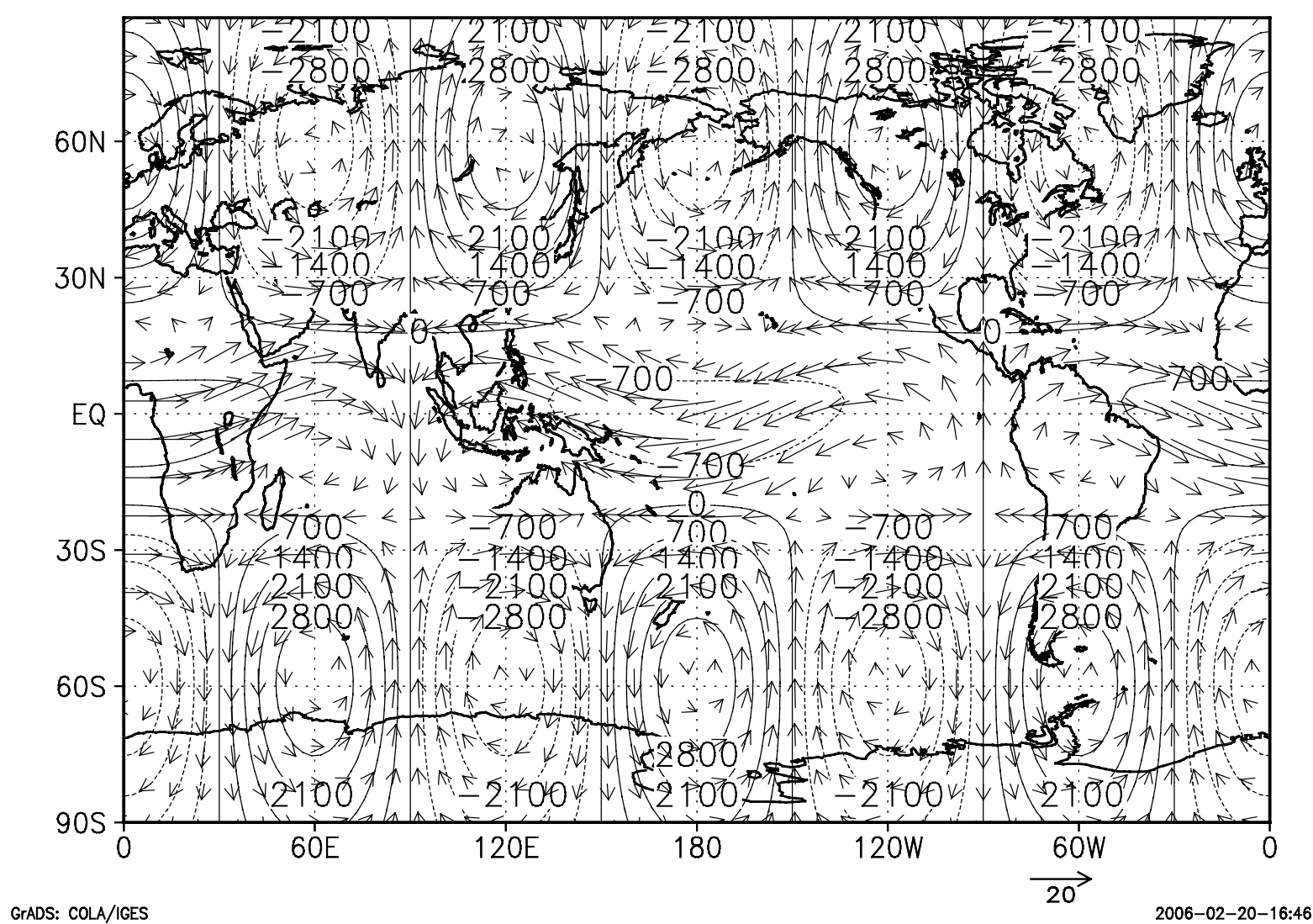

Figura 5.10: Distribuição horizontal dos campos do vento horizontal (vetor) e do geopotencial (contornos) em $\mathrm{p}=1000 \mathrm{hPa}$ associados à mesma solução mostrada na Figura 5.6b no instante inicial $(\mathrm{t}=0)$. Os campos do vento horizontal e do geopotencial são dados, respectivamente,

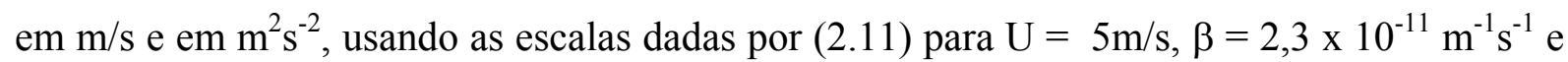
$\mathrm{L}=10^{6} \mathrm{~m}$.

Dessa forma, devido ao significativo acoplamento entre as ondas constituintes da tríade 6 da Tabela 4.5 a à possível relação desses modos com alguns aspectos observados acerca da circulação atmosférica de grande-escala, as trocas de energia entre esses modos mostradas na Figura 5.6, bem como as mudanças de regime na solução no espaço físico ilustradas nas Figuras 5.7 a 5.9 em decorrência dessas trocas, podem constituir um mecanismo potencialmente importante para a conexão trópicos-extratrópicos na escala intra-sazonal e/ou em escalas ainda mais longas. Os resultados aqui apresentados sugerem que o acoplamento ressonante entre a onda de Rossby barotrópica com número de onda zonal 3 e modo 
meridional $n=2$ e a onda de Kelvin com número de onda zonal 1 e a estrutura vertical do primeiro modo baroclínico $\mathrm{m}=1$, por meio da onda mista de Rossby-gravidade com a mesma estrutura vertical da onda de Kelvin e possuindo número de onda zonal 2, pode ser um possível mecanismo de ligação entre a atividade da OMJ nos trópicos e os padrões de teleconexões barotrópicos equivalentes em médias latitudes, tais como o PNA e o PSA.

Neste contexto, a dependência do período das trocas de energia nas tríades ressonantes exploradas neste capítulo com relação às amplitudes iniciais dos modos pode ter implicações para a previsibilidade das oscilações de baixa freqüência na atmosfera (oscilações intrasazonais e de período ainda mais longo). Os resultados mostrados neste capítulo destacam que o período das trocas de energia entre as ondas constituintes de uma determinada tríade ressonante, e conseqüentemente o período da vacilação da solução no espaço físico em decorrência dessas trocas, dependem da amplitude inicial das ondas que interagem. As amplitudes iniciais das ondas que interagem, por sua vez, são dadas pela projeção de um estado qualquer da atmosfera (que pode ser usado como condição inicial numa previsão de tempo ou de clima) nesses modos. Por outro lado, a projeção de um determinado estado da atmosfera num dado modo depende da maneira pela qual um modelo de previsão de tempo representa certos processos físicos. Por exemplo, a onda mista de Rossby-gravidade e a onda de Kelvin constituintes da tríade ressonante analisada na Figura 5.6 estão diretamente ligadas à convecção nos trópicos. Logo, num modelo atmosférico de previsão de tempo, a projeção de um estado da atmosfera nesses modos, e conseqüentemente o período da vacilação resultante da troca de energia entre esses modos, podem depender da representação desses processos convectivos úmidos, que é altamente dependente da parametrização convectiva.

De acordo com os resultados mostrados na Figura 5.6, a previsibilidade das oscilações de baixa freqüência na atmosfera pode ser alta quando a amplitude inicial da onda de Kelvin é muito maior que a das ondas de Rossby e de Yanai. Esta maior previsibilidade neste caso ocorre porque a solução das equações reduzidas para a tríade 6 da Tabela 4.5 torna-se mais estável em decorrência da inibição das trocas de energia entre os modos, com a solução se aproximando do regime linear. De fato, diversos trabalhos (Jones et al. 2000; 2004; Jones e Schemm 2000) foram realizados com o intuito de investigar a previsibilidade das oscilações intra-sazonais em modelos de circulação geral da atmosfera (GCMs) em diferentes fases da atividade da OMJ nos trópicos. Nesses estudos foram realizadas um conjunto de simulações numéricas perturbando-se a condição inicial em diferentes fases da OMJ e, de uma forma geral, tais trabalhos destacam que a previsibilidade na escala intra-sazonal nesses modelos é maior nas fases mais ativas da OMJ, i.e., quando a atividade da OMJ é mais intensa. 


\section{CAPÍTULO 6}

\section{Considerações Finais}

\subsection{Sumário e Conclusões Gerais do Trabalho}

No trabalho exposto nesta tese foram analisadas as interações não lineares fracas envolvendo ondas baroclínicas e equatorialmente confinadas e ondas de Rossby barotrópicas possuindo significativas amplitudes em médias e altas latitudes utilizando a versão adiabática das equações primitivas em coordenadas isobáricas e com a aproximação do plano $\beta$ equatorial. Estas interações não lineares onda-onda foram exploradas neste trabalho num contexto idealizado, com as ondas estando imersas numa atmosfera em repouso, horizontalmente homogênea e estavelmente estratificada na vertical. Além disso, o parâmetro de estabilidade estática deste estado básico foi suposto constante ao longo da troposfera com o intuito de simplificar a análise matemática. Supondo condições de fronteira rígidas na vertical, com a componente vertical do vento nula na superfície e num determinado topo de altura finita da troposfera, tais condições foram expandidas em séries de Taylor em torno de superfícies isobáricas num procedimento similar ao adotado na teoria de ondas de gravidade de superfície em águas profundas e na teoria de ondas de gravidade-capilares. Aplicando o método assintótico de múltiplas escalas temporais nas equações governantes no limite de pequenas amplitudes, as ondas equatoriais de Rossby, Poincaré (gravidade-inerciais), Yanai (mista de Rossby-gravidade) e de Kelvin, associadas a vários modos verticais, foram obtidas como soluções de ordem dominante. A partir da condição de solvabilidade do problema de ordem superior, foi obtido um modelo assintótico reduzido que governa a interação fracamente não linear entre as ondas numa única tríade ressonante. A conservação da energia total na mais baixa ordem implica que a soma dos coeficientes de interação num determinado tripleto ressonante deve ser nula. Em geral, foi verificado que as ondas cujos modos verticais satisfazem à relação $m= \pm \mathrm{j} \pm \mathrm{l}$ (ou similarmente $\lambda_{\mathrm{m}} \approx \pm \lambda_{\mathrm{j}} \pm \lambda_{1}$ ) possuem as mais expressivas interações triádicas, embora essa condição seja menos exclusiva que a condição de ressonância imposta para os números de onda zonal. Com isso, o foco do presente estudo foi direcionado somente aos tripletos ressonantes contendo duas ondas equatoriais internas com a mesma estrutura vertical e uma onda de Rossby barotrópica. Assim como ocorre em vários outros problemas físicos, nesses tripletos ressonantes aqui encontrados a onda com a maior 
freqüência absoluta num dado tripleto ressonante possui sempre o coeficiente de interação com o sinal oposto ao dos outros dois modos, agindo sempre como uma fonte ou como um sumidouro de energia para as demais componentes do tripleto ressonante.

Duas interações triádicas receberam atenção especial devido ao significativo acoplamento entre as ondas constituintes desses tripletos e à possível relação de alguns desses modos a alguns aspectos observados acerca da circulação atmosférica de grande-escala e baixa variabilidade. Uma dessas tríades é composta por uma onda de Rossby barotrópica com modo meridional $\mathrm{n}=2$ interagindo ressonantemente com uma onda de Yanai cuja estrutura vertical é dada pelo primeiro modo baroclínico $\mathrm{m}=1$, ambas possuindo o mesmo número de onda zonal $6(\mathrm{k} \approx 0,943)$, por meio de um modo geostrófico zonalmente simétrico $(\mathrm{k}=0)$ possuindo a mesma estrutura vertical da onda mista e modo meridional $n=1$. A outra tríade ressonante explorada é constituída por uma onda de Yanai com número de onda zonal 2 e uma onda de Kelvin com número de onda zonal 1, ambas possuindo a estrutura vertical do primeiro modo baroclínico $\mathrm{m}=1$, e uma onda de Rossby barotrópica possuindo número de onda zonal 3 e modo meridional $\mathrm{n}=2$. Em geral, o período das trocas de energia numa tríade ressonante é dependente das amplitudes iniciais dos modos. Contudo, para as amplitudes iniciais dos modos selecionadas de forma a caracterizarem magnitudes típicas de anomalias de tempo e clima observadas na atmosfera, foi verificado nas integrações das equações reduzidas para essas duas tríades ressonantes que os modos na primeira tríade tipicamente trocam energia na escala intra-sazonal, enquanto na segunda tríade os modos trocam energia tanto na escala intra-sazonal quanto numa escala semi-anual, dependendo da amplitude inicial do modo de Rossby, que constitui o modo menos energeticamente ativo nessa tríade ressonante. Assim, os resultados apresentados neste trabalho sugerem que essas duas interações triádicas ressonantes podem constituir mecanismos dinâmicos importantes para a conexão trópicosextratrópicos na escala de baixa freqüência dos movimentos atmosféricos (variabilidade intrasazonal e de período ainda mais longo). Inclusive, dada à possível relação da onda de Kelvin com número de onda zonal 1 constituinte da tríade ressonante 6 da Tabela 4.5 com a atividade da oscilação de Maden-Julian (OMJ) nos trópicos e à possível relação da onda de Rossby barotrópica possuindo número de onda zonal 3 constituinte dessa mesma tríade ressonante com os típicos padrões de número de onda 3 observados em médias latitudes relacionados aos padrões do PNA e do PSA, os resultados também sugerem que o acoplamento ressonante entre esses dois modos por meio da onda de Yanai baroclínica e com número de onda zonal 2 pode ser um possível mecanismo de conexão entre a OMJ nos trópicos e os padrões do PNA e do PSA. Neste contexto, a dependência da magnitude e do período das trocas de energia entre 
os modos constituintes dessa tríade ressonante com relação às amplitudes iniciais dos modos podem ter implicações para a previsibilidade das oscilações intra-sazonais na atmosfera.

Dessa forma, os resultados apresentados neste trabalho destacam que as interações triádicas ressonantes envolvendo os principais modos de variabilidade da circulação atmosférica de grande-escala podem constituir um possível mecanismo dinâmico responsável pela variabilidade de baixa freqüência dos movimentos atmosféricos, ou seja, um possível mecanismo dinâmico responsável pela variabilidade intra-sazonal e/ou pela variabilidade de período ainda mais longo da circulação atmosférica.

\subsection{Sugestões para Pesquisas Futuras}

A principal questão que surge a partir dos resultados obtidos no presente trabalho refere-se à como as ressonâncias aqui analisadas seriam modificadas com a inclusão dos efeitos da geometria esférica da Terra e da presença de uma estratificação vertical da atmosfera básica mais realista. Além disso, uma outra questão que precisa ser analisada futuramente é como a presença de um escoamento básico mais realista modificaria as interações triádicas ressonantes analisadas neste trabalho. De fato, os distúrbios ondulatórios na atmosfera, em geral, dificilmente estão imersos num estado básico em repouso. Conseqüentemente, a inclusão de um escoamento básico mais realista permitiria uma melhor comparação dos resultados teóricos aqui obtidos com as observações. Logo, seria interessante analisar o efeito de um escoamento básico em equilíbrio geostrófico e apresentando cisalhamentos tanto vertical quanto meridional sobre as interações não lineares ressonantes analisadas neste trabalho. Além disso, a inclusão de um campo básico mais realista nas equações governantes (2.1) e a análise das interações triádicas ressonantes nesse sistema podem levar a resultados interessantes com relação aos mecanismos de instabilidade não linear na atmosfera. Becker e Grimshaw (1993), usando as equações de Boussinesq representativas da dinâmica de grande-escala do oceano, mostram que na presença de um escoamento básico com um cisalhamento vertical é possível a existência de tríades ressonantes explosivas. Becker e Grimshaw adotam um formalismo variacional num sistema de coordenadas Lagrangeano se movendo com a velocidade do campo básico e mostram que uma determinada tríade ressonante torna-se explosiva quando o campo básico apresenta uma camada crítica, onde a velocidade de fase do modo de maior freqüência absoluta iguala-se à velocidade do campo básico. É importante ressaltar que esse fenômeno de interações 
ressonantes explosivas pode ocorrer mesmo se o escoamento básico for linearmente estável, ou seja, mesmo se as freqüências dos modos normais forem reais.

Um outro ponto que precisa ser abordado futuramente refere-se ao impacto do termo de aquecimento/resfriamento diabático na equação da termodinâmica, não considerado neste estudo, bem como o efeito de um acoplamento com a camada limite planetária, sobre as ressonâncias obtidas e analisadas neste trabalho. O acoplamento com a camada limite planetária modificaria a condição de fronteira na superfície. Neste caso, a condição de fronteira vertical inferior seria dada pela velocidade vertical no topo da camada limite planetária, que por sua vez seria determinada por meio de uma parametrização dos processos turbulentos nesta camada limite. No caso do termo diabático, através da parametrização desse efeito em termos das variáveis dependentes das equações (2.1), é possível mostrar que este termo diabático também pode acoplar ressonantemente as ondas obtidas como soluções de ordem dominante do modelo atmosférico aqui adotado por meio da ressonância das ondas com este aquecimento. Este mecanismo de acoplamento ressonante entre ondas por meio do aquecimento diabático é similar ao mecanismo de interações entre ondas por meio da topografia explorado por Majda et al. (1999) no contexto das equações da água-rasa no plano $\beta$-equatorial. Inclusive, uma vez que o aquecimento diabático associado à típica convecção profunda nos trópicos apresenta uma estrutura vertical ressonante com o primeiro modo baroclínico, este aquecimento diabático pode ter um papel similar ao modo geostrófico zonalmente simétrico com a estrutura vertical do primeiro modo baroclínico, acoplando ressonantemente as ondas de Rossby barotrópicas e as ondas de Yanai internas exploradas neste trabalho. Assim, uma outra sugestão para pesquisa futura seria analisar as possíveis interações ressonantes entre as ondas na atmosfera por meio do aquecimento diabático. 


\section{REFERÊNCIAS}

ABRAMOWITZ, M.; I.A. STEGUN (eds) (1964). Handbook of mathematical functions. U. S. Govt. Printing Office, Washington, D. C.

BECKER, J. M.; R. H. J. GRIMSHAW. Explosive resonant triads in a continuously stratified shear flow. J. Fluid Mech., 257, 219-228, 1993.

BLACKMON, M. L.; Y. H. LEE; J. M. WALLACE. Horizontal structure of 500mb height fluctuations with long, intermediate and short time scales as deduced from lag-correlation statistics. J. Atmos. Sci, 41, 961-979, 1984.

BRETHERTON, F. P. Resonant interactions between waves. The case of discrete Oscillations. J. Fluid. Mech., 20, 457-479, 1964.

BUTKOV, E. Mathematical Physics. Addison-Wesley Publishing Company, 1968.

CARVALHO, L. M. V.; C. JONES; T. AMBRIZZI. Opposite phases of the Antarctic oscillation and relationships with intraseasonal to interannual activity in the tropics during the austral summer. J. Climate, 18, 702-718, 2005.

CASE, K. M.; S. C. CHIU. Three-wave resonant interactions of gravity-capillary waves. Phys. Fluids., 20, 742-745, 1977.

CRAIK, A. D. D. Wave interactions and fluid flows. Cambridge Monographs on Mechanics and Applied Math. Cambridge University Press., pp. 322, 1985.

DEMARIA, M. Linear response of a stratified tropical atmosphere to convective forcing. $\mathbf{J}$. Atmos. Sci., 42(18), 1944-1959, 1985.

DOMARACKI, A.; A. Z. LOESCH. Nonlinear interactions among equatorial waves. J. Atmos. Sci., 34, 486-498, 1977. 
DUFFY, D. G. Resonant interactions of inertio-gravity and Rossby waves. J. Atmos. Sci., 31, 1218-1231, 1974.

FREDERIKSEN, J. S. Genesis of intraseasonal oscillations and equatorial waves. J. Atmos. Sci., 59 (19), 2761-2780, 2002.

FREDERIKSEN, J. S. A unified three-dimensional instability theory of the onset of blocking and cyclogenesis. J. Atmos. Sci., 39, 969-987, 1982.

FREDERIKSEN, J. S. The onset of blocking and cyclogenesis: Linear theory. Aust. Meteor. Mag. , 31, 15-26, 1983a.

FREDERIKSEN, J. S. Disturbances and eddy fluxes in the northern hemisphere flows: Instability of three-dimensional January and July flows. J. Atmos. Sci., 40, 836-855, 1983 b.

FREDERIKSEN, J. S. A unified three-dimensional instability theory of the onset of blocking and cyclogenesis. Part II: Teleconnection patters. J. Atmos. Sci., 40, 2593-2609, 1983c.

FULTON, S. R.; W. H. SCHUBERT. Vertical normal mode transforms: Theory and application. Mon. Wea. Rev., 113, 647-658, 1985.

GEISLER, J. E.; D. E. STEVENS. On the vertical structure of damped steady circulation in the tropics. Quart. J. Roy. Meteor. Soc., 108, 87-94, 1982.

GHIL, M.; K. C. MO. Intraseasonal oscillations in the global atmosphere. Part II: Southern Hemisphere. J. Atmos. Sci., 48, 780-790, 1991.

GRIMM, A. M.; P. L. SILVA DIAS. Use of barotropic models in the study of the extratropical response to tropical heat sources. J. Meteor. Soc. Japan, 73, 1037-1049, 1995a.

GRIMM, A. M.; P. L. SILVA DIAS. Analysis of tropical-extratropical interactions with influence functions of a barotropic model. J. Atmos. Sci., 52 (20), 3538-3555, 1995b. 
HAYASHI, Y. A theory of large-scale equatorial waves generated by condensational heat and accelerating the zonal wind. J. Meteor. Soc. Japan, 48, 140-160, 1970.

HAYASHI, Y.; A. SUMI. The 30-40 day oscillation simulated in an "aqua planet" model. J. Meteor. Soc. Japan, 64, 4451-467, 1986.

HENDON, H.; M. SALBY. The life cycle of the Madden-Julian oscillation. J. Atmos. Sci., 51, 2225-2237, 1994.

HIGGINS, R. W.; K. C. MO. Persistent North Pacific circulation anomalies and the tropical intra-seasonal oscillation. J. Climate., 10, 223-244, 1997.

HOREL, J. D.; J. M. WALLACE. Planetary-scale atmospheric phenomena associated with the Southern Oscillation. Mon. Wea. Rev.,109, 813-829, 1981.

HOSKINS., B. J.; A. J. SIMMONS; D. G. ANDREWS. Energy dispersions in a barotropic atmosphere. Quart. J. R. Met. Soc., 103, 553-567, 1977.

HOSKINS, B.J.; T. AMBRIZZI. Rossby wave propagation on a realistic longitudinally varying flow. J. Atmos. Sci., 50, 1661-1671, 1993.

HOSKINS, B.J.; D.J KAROLY. The steady linear response of a spherical atmosphere to thermal and orographic forcing., J. Atmos. Sci., 38, 1179-1196, 1981.

JACOBS, S. J.; A. WINN-NIELSEN. On the stability of a barotropic basic flow in a stratified atmosphere. J. Atmos. Sci., 23, 682-687, 1966.

JONES, C.; B. C. WEARE. The role of low-level moisture convergence and the ocean latent heat fluxes in the Madden and Julian Oscillation: an observational analysis using ISCCP data and ECMWF analysis. J. Climate, 9, 3086-3104, 1996. 
JONES, C.; D. E. WALISER; C. GAUTIER. The influence of the Madden and Julian Oscillation on ocean surface heat fluxes and high sea surface temperature variability in the warm pool region. J. Climate, 11, 1057-1072, 1998.

JONES, C.; D. E. WALISER; K. M. LAU; W. STERN. The Madden-Julian Oscillation and its impact on Northern Hemisphere weather predictability. Mon. Wea. Rev., 132, 1462-1471, 2004.

JONES, C.; D. E. WALISER; K. M. LAU; W. STERN. Global occurrences of extreme precipitation and the Madden-Julian Oscillation: observations and predictability. J. Climate, 17, 4575-4589, 2004.

JONES, C.; J-K. E. SCHEMM. The influence of intraseasonal variations on medium- to extended-range weather forecasts over South America. Mon. Wea. Rev., 128, 486-494, 2000.

KALNAY, E.; CO-AUTORES. The NCEP/NCAR 40-year Reanalysis Project. Bull. Amer. Meteor. Soc., 77, 437-471, 1996.

KAROLY, D. J. Southern Hemisphere circulation features associated with El Nino-Southern Oscillation. J. Climate, 2, 1239-1252, 1989.

KASAHARA, A; SILVA DIAS, P. L. Response of equatorial planetary waves to stationary tropical heating in the global atmosphere with meridional and vertical shear. J. Atmos. Sci., 43 (18), 1893-1911, 1986.

KASAHARA, A; K. PURI. Spectral representation of the three-dimensional global data by expansion in normal modes functions. Mon. Wea. Rev., 109, 37-61, 1981.

KESSLER, W. S.; M. J. MCPHADEN; K. M. WHEICKMANN. Forcing of intraseasonal Kelvin waves in the equatorial Pacific. J. Geophys. Res., 100, 10613-10631, 1996.

KIDSON, J. W. Interannual variations in the Southern Hemisphere circulation. J. Climate, 1, 1177-1198, 1988. 
KIDSON, J. W. Intraseasonal variation in the Southern Hemisphere circulation. J. Climate, 4, 939-953, 1991.

KRISHNAMURTI, T. N.; D. R. CHAKRABORTY. The dynamics of phase locking. J. Atmos. Sci., 62, 2952-2964, 2005.

LAU, K-M.; J. S. BOYLE. Tropical and Extratropical forcing of the large-scales circulation: a diagnostic study. Mon. Wea. Rev., 115, 400-428, 1987.

LAU, N.; T. J. PHILLIPS. Coherent fluctuations of extratropical geopotential height and tropical convection in intra-seasonal time-scales. J. Atmos. Sci., 43, 1164-1181, 1986.

LAU, K-M.; L. PENG. Origin of low-frequency (intraseasonal) oscillations in the tropical atmosphere. Part I: Basic theory. J. Atmos. Sci., 44, 950-972, 1987.

LAU, K-M.; P. H. CHAN. Aspects of the 40-50 day oscillation during the northern summer as inferred from outgoing longwave radiation. Mon. Wea. Rev., 114, 1354-1367, 1986.

LIEBMANN, B.; D. L. HARTMANN. An observational study of tropical-midlatitude interaction on intra-seasonal time-scales during winter. J. Atmos. Sci., 41, 3334-3350, 1984.

LIM, H.; C-P. CHANG. Dynamics of teleconnections and walker circulations forced by equatorial heating. J. Atmos. Sci., 40, 1897-1915, 1983.

LIM, H.; C-P. Chang. Generation of internal- and external- mode motions from internal heating: effects of vertical shear and damping. J. Atmos. Sci., 43 (9), 948-957, 1986.

LINDZEN, R.S. Planetary waves on beta-planes. Mon. Wea. Rev., 95, 441-451, 1967.

LOESCH, A. Z.; R. C. DEININGER. Dynamics of closed systems of resonantly interacting equatorial waves. J. Atmos. Sci., 36, 1490-1497, 1979. 
LONGUET-HIGGINS, M. S.; A. E. Gill. Resonant interactions between planetary waves. Proc. Roy. Soc. London., A299, 120.

LORENZ, E. N. The mechanics of vacillation. J. Atmos. Sci., 20, 448-464, 1963.

MADDEN, R. A.; P. JULIAN. Observations of the 40-50 day tropical oscillation - A review. Mon. Wea. Rev., 122, 814-836, 1994.

MAGAÑA, V.; M. YANAI. Tropical-middle-latitude interactions on the time scale of 30 to 60 days during the northern summer of 1979. J. Climate, 4 , 180-201, 1991.

MAGAÑA, V.; M. YANAI. Mixed Rossby-gravity waves triggered by lateral forcing. J. Atmos. Sci, 52 (9), 1473-1486, 1995.

MAHONEY, D. J. On wave interactions, I: Explosive resonant triads. Studies in Applied Math., 95:381-417, 1995.

MAJDA, A. J.; J. A. BIELLO. The nonlinear interaction of barotropic and equatorial baroclinic Rossby waves. J. Atmos. Sci., 60, 1809-1821, 2003.

MAJDA, A. J. Introduction to PDEs and Waves for the Atmosphere and Ocean. Courant Institute Lecture Series 9, American Mathematical Society, 2003.

MAJDA, A. J.; R. R. ROSALES; E. G. TABAK; C. V. TURNER. Interaction of long-scale equatorial waves and dispersion of Kelvin waves through topographic resonances. J. Atmos. Sci., 56, 4118-4133, 1999.

MAK, M.-K. Laterally driven stochastic motions in the tropics. J. Atmos. Sci, 26, 41-64, 1969.

MALONEY, E. D.; D. L. HARTMANN. Modulation of eastern north Pacific hurricanes by the Madden-Julian oscillation. J. Climate, 13 (9), 1451-1460, 2000. 
MARUYAMA, T. Large-scale disturbances in the equatorial lower stratosphere. J. Meteor. Soc. Japan, 45, 391-408, 1967.

MATSUNO, T. Quasi-geostrophic motions in the equatorial area. J. Meteor. Soc. Japan, 44, 25-43, 1966.

MCGOLDRIK, L. F. Resonant interactions among capillary-gravity waves. J. Fluid. Mech., 21, 305-331, 1965.

MILEWSKI, P.; J. B. KELLER. Three-dimensional water waves. Studies in Applied Math., 37:000-000, 1996.

MILEWSKI, P.; E. G. TABAK. A reduced model for nonlinear dispersive waves in a rotating environment. Geophys. Astrophys. Fluid Dynamics., 90, 139-159, 1999.

MO, K. C.; R. E. LIVEZEY. Tropical-Extratropical geopotential height teleconnections during the northern hemisphere winter. Mon. Wea. Rev., 114, 2488-2515, 1986.

MO, K. C.; R. W. HIGGINS. The Pacific-South American modes and tropical convection during the southern hemisphere winter. Mon. Wea. Rev., 126, 1581-1596, 1998.

MO, K. C.; G. H. WHITE. Teleconnections in the Southern Hemisphere. Mon. Wea. Rev., $113,22-37,1985$.

PALMER, T. N. Large-scale tropical-extratropical interactions on time-scales of a few days to a season. Aust. Meteor. Mag., 36, 107-125, 1988.

PHILLIPS, O. M. On the dynamics of unsteady gravity waves of finite amplitude. J. Fluid Mech., 9, 193-217, 1960.

PHILlIPS, O. M. On internal wave interactions. Proc. $6^{\text {th }}$ Naval Hydrodynamics Symposium., Washington D. C., Paper 21, 1966. 
PIRES, P.; J. L. REDELSPERGER; J. P. LAFORE. Equatorial atmospheric waves and their association to convection. Mon. Wea. Rev., 125 (6), 1167-1184, 1997.

RANDEL, W. J. Upper tropospheric equatorial waves in ECMWF reanalysis. Quart. J. R. Met. Soc., 118, 365-394, 1992.

RAUPP, C. F. M. (2002). Efeitos de processos não lineares na influência inter-hemisférica de fontes de calor. São Paulo, 2002. p. [Dissertação de Mestrado. Instituto de Astronomia, Geofísica e Ciências Atmosféricas/Universidade de São Paulo].

RAUPP, C. F. M.; P. L. SILVA DIAS. Resonantly interacting equatorial waves. Tellus, 58A (2), 2006. (Aceito para Publicação).

RAUPP, C. F. M.; P. L. SILVA DIAS. Excitation mechanism of mixed Rossby-gravity waves in the equatorial atmosphere: role of the nonlinear interactions among equatorial waves. $\mathbf{J}$. Atmos. Sci., 62, 1446-1462, 2005.

RIPA, P. On the theory of nonlinear interactions among geophysical waves. J. Fluid Mech., 103, 87-115, 1981.

RIPA, P. Weak interactions of equatorial waves in a one-layer model. Part I: General Properties., J. Phys. Oceanogr., 13, 1208-121226, 1983 a.

RIPA, P. Weak interactions of equatorial waves in a one-layer model. Part II: Applications., J. Phys. Oceanogr., 13, 1227-1240, 1983 b.

ROGERS, J. C.; H. VAN LOON. Spatial variability of sea level pressure and height anomalies over the Southern Hemisphere. Mon. Wea. Rev., 110, 1375-1392, 1982.

ROSSBY, C. G. On the propagation of frequencies and energy in certain types of oceanic and atmospheric waves. J. Meteor., 2, 187-204, 1945. 
SANTOS, I. A.; P. L. SILVA DIAS; A. R. TORRES. The role of mixed Rossby-gravity waves on the organization of convection in the Amazon., Anais: Congresso Brasileiro de Meteorologia, 12., Foz do Iguaçu - PR, 2002.

SILVA DIAS, P.L.; W. H. SCHUBERT. The dynamics of equatorial mass-flow adjustment. Atmospheric Science Paper No. 312 (Department of Atmospheric Science Colorado State University), Fort Collins, Colorado, USA, 1979.

SILVA DIAS, P. L.; J. P. BONATTI. A preliminary study of the observed modal structure of the Summer circulation over tropical South America. Tellus, 37A, 185-195, 1985.

TAKAYABU, Y. Large-scale cloud disturbance associated with equatorial waves. Part I: Spectral features of the cloud disturbances. J. Meteor. Soc. Japan, 72 (3), 433-448, 1994.

WALLACE, J.M.; D.S. GUTZLER. Teleconnection in the geopotential heigth field during the Northern Hemisphere winter. Mon. Wea. Rev., 109, 785-812, 1981.

WALLACE, J. M.; V. E. KOUSKY. Observational evidence of Kelvin waves in the tropical stratosphere. J. Atmos. Sci., 25, 900-907, 1968.

WANG, B.; X. XIE. Low-frequency equatorial waves in vertically sheared zonal flows. Part I: Stable waves. J. Atmos. Sci., 53 (3), 449-467, 1996.

WANG, B.; P. WEBSTER; H. TENG. Antecedents and self-induction of active-break south Asian monsoon unraveled by satellites. Geophysical Research Letters, 32, L04704, 2005.

WEBSTER, P.J. Response of the tropical atmosphere to local steady forcing. Mon. Wea. Rev., 100, 518-540, 1972.

WEBSTER, P.J. Mechanisms determining the atmospheric response to sea surface temperature anomalies. J. Atmos. Sci., 38, 554-571, 1981. 
WEBSTER, P.J. Seasonality in the local and remote atmospheric response to the sea surface temperature anomalies. J. Atmos. Sci., 39, 41-52, 1982.

WHEICKMANN, K. M. El Niño/Southern Oscillation and Madden-Julian (30-60 day) oscillations during 1981/1982. J. Geophys. Res., 96, 3187-3195, 1991.

WEILAND, J.; H. WILHELMSSON. Coherent Nonlinear Interaction of Waves in Plasmas. Oxford: Pergamon. 1977.

WHEELER, M.; G. N. KILADIS. Convectively coupled equatorial waves: Analysis of clouds and temperature in the wavenumber-frequency domain. J. Atmos. Sci., 56, 374-399, 1999.

WHEELER, M.; H. H. HENDON. An all season real time multivariate MJO index: development of an index for monitoring and prediction. Mon. Wea. Rev., 132, 1917-1932, 2004.

WIIN-NIELSEN, A. On the motion of various vertical modes of transient very long waves. Part I: $\beta$-plane approximation. Tellus, 23, 87-98, 1971.

YANAI, M.; S. ESBENSEN; J-H. CHU. Determination of bulk properties of tropical cloud clusters from large-scale heat and moisture budgets. J. Atmos. Sci., 30, 611-627, 1973.

YANAI, M.; T. MARUYAMA. Stratospheric wave disturbances propagating over the equatorial Pacific. J. Meteor. Soc. Japan, 44, 291-294, 1966.

ZHANG, C. Atmospheric intraseasonal variability at the surface in the tropical western Pacific Ocean. J. Atmos. Sci., 53, 739-758, 1996. 


\section{APÊNDICE A}

\section{Determinação dos Perfis de Temperatura e Densidade do Estado Básico}

O parâmetro de estabilidade estática do estado básico na equação da Termodinâmica (2.3) é definido a partir de (2.2) pela seguinte expressão

$$
\bar{\sigma}=\frac{R}{p}\left(\frac{R \bar{T}}{p C_{p}}-\frac{d \bar{T}}{d p}\right)
$$

onde $\mathrm{R}$ representa a constante dos gases para o ar seco, $\mathrm{C}_{\mathrm{p}}$ refere-se à capacidade térmica do ar seco à pressão constante e $\overline{\mathrm{T}}=\overline{\mathrm{T}}(\mathrm{p})$ corresponde à temperatura do estado básico, caracterizado por uma atmosfera em repouso, horizontalmente homogênea, e em equilíbrio hidrostático. Logo, supondo $\bar{\sigma}$ constante, a temperatura $\overline{\mathrm{T}}$ do estado básico satisfaz à seguinte equação diferencial ordinária:

$$
p \frac{d \bar{T}}{d p}-\kappa \bar{T}+\frac{\bar{\sigma}}{R} p^{2}=0
$$

sendo $\kappa=R / C_{p}$. Para resolver a equação (A.2) acima, o método aqui empregado consiste em buscar inicialmente a solução da parte homogênea, que é obtida supondo nulo o último termo de (A.2). Neste caso, a solução homogênea de (A.2) é dada por

$$
\bar{T}(p)=C\left(\frac{p}{p_{0}}\right)^{\kappa}
$$

onde C é uma constante arbitrária. Em seguida, para resolver a equação (A.2) supõe-se uma solução na forma dada por (A.3) mas com o coeficiente $C$ variável, ou seja, $C=C(p)$. Neste caso, substituindo (A.3) com o coeficiente C variável em (A.2), obtém-se:

$$
\frac{d C}{d p}=-\frac{\bar{\sigma}}{R} p_{0}{ }^{\kappa} p^{1-\kappa}
$$


De (A.4), segue que

$$
C(p)=-\frac{\bar{\sigma}}{R} p_{0}{ }^{\kappa} \frac{p^{2-\kappa}}{2-\kappa}+A
$$

sendo A uma constante arbitrária. Substituindo (A.5) em (A.3), obtém-se a solução geral de (A.2) para $\overline{\mathrm{T}}(\mathrm{p})$ :

$$
\bar{T}(p)=-\frac{\bar{\sigma}}{R} \frac{p^{2}}{2-\kappa}+A\left(\frac{p}{p_{0}}\right)^{\kappa}
$$

Uma vez que a constante A é arbitrária, esta pode ser ajustada de forma a se obter valores realistas de temperatura (na escala absoluta, i.e., em K). Assim, para $\bar{\sigma}=2 \times 10^{-6} \mathrm{~m}^{4}$ $\mathrm{s}^{2} \mathrm{Kg}^{-2}$ e $\mathrm{A}=330$, obtém-se o perfil de temperatura ilustrado na Figura 2.1. O perfil de densidade $\bar{\rho}(\mathrm{p})$ é determinado a partir de $\overline{\mathrm{T}}(\mathrm{p})$ usando a equação do estado para o ar seco, i.e.,

$$
\bar{\rho}(p)=\frac{p}{R \bar{T}(p)}
$$

Finalmente, o perfil de $\frac{d \bar{\rho}}{d p}$, necessário para o cálculo dos coeficientes de interação em (2.92), é obtido derivando a equação (A.7) com relação a p e usando (A.2). A expressão final é dada por

$$
\frac{d \bar{\rho}}{d p}=\frac{1}{R \bar{T}^{2}}\left(\bar{T}-\kappa \bar{T}+\frac{\bar{\sigma}}{R} p^{2}\right)
$$




\section{APÊNDICE B \\ Equação da Estrutura Vertical (2.31) para o Caso $\lambda=0$}

Uma vez que o parâmetro de estabilidade estática do estado básico dado por $\bar{\sigma}$ é constante, a equação (2.31) para o autovalor $\lambda=0$ é dada por

$$
\frac{d^{2} G}{d p^{2}}=0
$$

Logo, $G(p)$ neste caso pode ser escrita como:

$$
\mathrm{G}(\mathrm{p})=\mathrm{A}+\mathrm{Bp}
$$

sendo A e B constantes arbitrárias. Substituindo (B.2) nas condições de fronteira (2.33), segue que

$$
\widetilde{\sigma}\left[A\left(\widetilde{\bar{\rho}}_{0}-\widetilde{\bar{\rho}}_{T}\right)+B\left(\widetilde{\bar{\rho}}_{0}-\widetilde{\bar{\rho}}_{T} \widetilde{p}_{T}\right)\right]=0
$$

De (B.3) resulta imediatamente que $\mathrm{A}=0$. Conseqüentemente, para que exista solução de (B.1) diferente da solução trivial é necessário que $\frac{\widetilde{\bar{\rho}}_{0}}{\widetilde{\bar{\rho}}_{T}}=\widetilde{p}_{T}$, ou alternativamente, $\mathrm{p}_{0} \overline{\bar{\rho}}_{0}=$ $\mathrm{p}_{\mathrm{T}} \bar{\rho}_{\mathrm{T}}$. Como esta condição é inviável para a atmosfera, ou seja, não tem nenhum significado físico, a única solução possível de (B.1) que satisfaz à condição de fronteira (2.33) é dada por $\mathrm{G}(\mathrm{p})=0$. Logo, o autovalor $\lambda=0$ é desprezado. 


\section{APÊNDICE C}

\section{Derivada das Autofunções da Estrutura Meridional do Problema de O(1)}

Derivando a expressão (2.45) referente às autofunções $\xi_{\mathrm{a}}(\mathrm{y})$ e usando a fórmula de derivação dos polinômios de Hermite (Butkov 1968), obtém-se a seguinte expressão para a derivada de primeira ordem das autofunções $\xi_{\mathrm{a}}(\mathrm{y})$ :

$$
\frac{d \xi_{a}}{d y}=\frac{1}{\sqrt{c_{a}}}\left[\begin{array}{ccc}
c_{a} & 0 & 0 \\
0 & c_{a} & 0 \\
0 & 0 & c_{a}{ }^{2}
\end{array}\right] \frac{d \xi_{a}}{d \hat{y}}
$$

onde $\hat{y}=\frac{y}{\sqrt{c_{a}}} \mathrm{e}$

$$
\frac{d \xi_{a}}{d \hat{y}}=-\hat{y} \xi_{a}(\hat{y})+\left[\begin{array}{c}
(n+1)\left(\widetilde{\varpi}_{a}-\widetilde{k}_{a}\right) H_{n}(\hat{y})+2 n(n-1)\left(\widetilde{\varpi}_{a}+\widetilde{k}_{a}\right) H_{n-2}(\hat{y}) \\
2 i n\left(\widetilde{\varpi}_{a}^{2}-\widetilde{k}_{a}^{2}\right) H_{n-1}(\hat{y}) \\
(n+1)\left(\widetilde{\varpi}_{a}-\widetilde{k}_{a}\right) H_{n}(\hat{y})-2 n(n-1)\left(\widetilde{\varpi}_{a}+\widetilde{k}_{a}\right) H_{n-2}(\hat{y})
\end{array}\right] \frac{e^{-\frac{\hat{y}^{2}}{2}}}{\left\|\xi_{a}\right\|}
$$

para $n \geq 0$. No caso do modo de Kelvin $(n=-1)$, a derivada também é dada por (C.1), mas com

$$
\frac{d \xi_{m, k,-1,3}}{d \hat{y}}=-\hat{y} \xi_{m, k,-1,3}(\hat{y})
$$

Da mesma maneira, derivando a expressão (2.51) em relação a y e usando a fórmula de derivação dos polinômios de Hermite, segue que a derivada das autofunções referente aos modos geostróficos zonalmente simétricos $(\mathrm{k}=0)$ é dada por (C.1) com

$$
\frac{d \xi_{m, 0, n, 1}(\hat{y})}{d \hat{y}}=-\hat{y} \xi_{m, 0, n, 1}(\hat{y})+\left\{\begin{array}{c}
\frac{n\left[-2(n+1) H_{n}(\hat{y})+4(n+1)(n-1) H_{n-2}(\hat{y})\right]}{\left[2^{n}(2 n+1) n ! \sqrt{\pi} 4 n(n+1)\right]^{\frac{1}{2}}} \\
0 \\
\frac{n\left[-2(n+1) H_{n}(\hat{y})-4(n+1)(n-1) H_{n-2}(\hat{y})\right]}{\left[2^{n}(2 n+1) n ! \sqrt{\pi} 4 n(n+1)\right]^{\frac{1}{2}}}
\end{array}\right\} e^{-\frac{\hat{y}^{2}}{2}}
$$




\title{
Dynamics of resonantly interacting equatorial waves
}

\author{
By CARLOS F. M. RAUPP* and PEDRO L. SILVA DIAS, Department of Atmospheric Sciences, \\ Institute of Astronomy, Geophysics and Atmospheric Sciences, University of São Paulo, São Paulo/SP, Brazil
}

(Manuscript received 24 August 2004; in final form 30 April 2005)

\begin{abstract}
In this paper we explore some dynamical features on the non-linear interactions among equatorial waves. The shallowwater equation model with the equatorial $\beta$-plane approximation is used for this purpose. The Galerkin method is applied to the governing equations with the basis functions given by the eigensolutions of the linear problem. From the phase space expansion of two particular integrals of motion of the system, quadratic to lowest order, some constraints are obtained which the coupling coefficients must satisfy in order to ensure the invariance of such integrals. From the numerical evaluation of the coupling coefficients, these constraints are used to determine the possible resonant triads among equatorial waves. Numerical integrations of the resonant three-wave problem show that the energy of the waves in a resonant triad evolves periodically in time, with the period and amplitude of the energy oscillations dependent on the magnitude of the initial amplitudes of the waves and the way in which the initial energy is distributed among the triad components. The high-frequency modes are found to be energetically more active than the low-frequency modes. The latter tend to act as 'catalytic' components in a resonant triad. Integrations of the problem of two resonant triads coupled by a single mode point out the importance of gravity waves in the intertriad energy exchanges, suggesting the significance of these modes in the redistribution of energy throughout the atmospheric motion spectrum. The results also show that the intertriad energy exchanges provided by the highest frequency mode of two triads occur in a longer time-scale than the intratriad interactions. Therefore, these results also suggest the importance of the high-frequency modes in the generation of the low-frequency variability (intraseasonal and even longer term) of the atmospheric flow.
\end{abstract}

\section{Introduction}

The equatorial trapping of large-scale wave-like disturbances is a prominent characteristic of the atmospheric circulation. This phenomenon was theoretically discovered by Matsuno (1966), who derived a complete set of linear wave-mode solutions of the shallow-water equations on the equatorial $\beta$-plane. After this theoretical finding, several studies have provided observational evidence of the existence of these waves in the equatorial atmosphere (Yanai and Maruyama, 1966; Maruyama, 1967; Gruber, 1974; Zangvil and Yanai, 1980; Takayabu, 1994; Dunkerton and Baldwin, 1995; Magaña and Yanai, 1995; Pires et al., 1997; Wheeler and Kiladis, 1999). As a consequence, the equatorially trapped waves have been applied in explaining fundamental features of tropical climate. The phenomena that have been explained in terms of equatorial wave theory include Hadley-Walker circulation (Webster, 1972; Gill, 1980; Lau and Lim, 1982; Lim and Chang, 1983), atmospheric tele-

\footnotetext{
* Corresponding author.

e-mail: cfmraupp@model.iag.usp.br

Postal address: Rua do Matão, 1226, Cidade Universitária, São PauloSP, Brazil 05508-090.
}

connection patterns (Lim and Chang, 1983; Raupp and Silva Dias, 2004), the low-frequency oscillation initially observed by Madden and Julian (1972) (Lau and Peng, 1987; Wang and Rui, 1990) and the El Niño Southern Oscillation (Lau, 1981; Hirst, 1986).

However, although a significant advance has been obtained on equatorial waves, especially on their interaction with moist convection, the non-linear behavior is far from completely understood. Particularly, most theoretical studies are based on linearized equations about simplified basic states and the non-linear dynamics of equatorial waves needs to be better understood. Despite the observational evidence of wave-wave interactions in the tropics (Kanamitsu et al., 1972), not many analytical works have been published on this matter. Domaracki and Loesch (1977) first studied resonant triads of equatorial waves using the asymptotic method of multiple scales. They obtained energy solutions which show that the triad member having the maximum absolute frequency always grows (or decays) at the expense of the other two triad members. Loesch and Deininger (1979) extended the results of Domaracki and Loesch for resonantly interacting waves in coupled triad configurations and also pointed out the importance of the maximum absolute frequency modes in individual triads for energy transfers throughout the system. 
On the other limit of energy scale, Salmon (1980) extended the results of geostrophic turbulence (Charney, 1971) (mainly, the concepts of energy and enstrophy conservation) to low latitudes.

Ripa (1981) formulated the non-linear wave-wave interaction problem for barotropic non-divergent Rossby waves and internal gravity waves on a vertical plane. Ripa applied Galerkin formalism with the basis functions given by the eigensolutions of the linear problem and showed that the conservation of two particular integrals of motion, quadratic to lowest order, leads to interesting properties that the coupling coefficients must satisfy in order to ensure the invariance of such integrals. This formalism was extended to the equatorial wave-guide in Ripa (1982, 1983a,b). Recently, Majda et al. (1999) analyzed the role of topography in resonantly coupling equatorial waves. They used an asymptotic expansion in terms of multiple scales and showed that by resonance with topography the equatorial waves can also interact with each other and exchange energy. Especially, the Kelvin wave can excite large-scale Yanai and Rossby waves with specific zonal wavelengths and slow down its phase speed by this resonance mechanism.

A straightforward view of Ripa's formalism will be carried out here in order to organize the theoretical framework. In this paper we used the results of Ripa's formalism to determine the possible resonant triads among equatorial waves and analyzed their dynamics by numerical integrations of the model equations in the phase space. The main concern here is to highlight the implications of the results for tropical atmospheric dynamics. It shall be shown that the resonant energy exchanges among equatorial waves have interesting implications for tropical atmospheric dynamics. Particularly, the results of the numerical integrations of the problem of two resonant triads coupled by a single mode suggest the importance of gravity waves (and, thus, moist convection) in the generation of the low-frequency (intraseasonal and/or even longer term) variability of the atmospheric circulation. Thus, as shall be discussed in Section 6, this paper provides an alternative explanation for the origin of intraseasonal oscillations in the tropics. In Section 2, the governing equations, the spectral method of solution and the concept of resonance in non-linear interactions are presented. In Section 3 we describe a straightforward view of Ripa's formalism on non-linear wave-wave interactions on the equatorial wave-guide. In Section 4 the main result of this formalism is used to determine the possible resonant triads among equatorial waves by numerical computation of the coupling coefficients. In Section 5 we explore the dynamics of the energy exchanges among equatorial waves in resonant triads by numerical integrations of the model equations in spectral space. The results of both the three-wave problem and the problem of two triads coupled by a single mode are shown. A summary of the results obtained in the previous sections and the implications of these results for the tropical atmospheric dynamics are presented in Section 6.

\section{Model equations and solution method}

The shallow-water model on the equatorial $\beta$-plane adopted in the present work can be described by the following system of equations in the vector form:

$\frac{\partial \xi}{\partial t}+\Omega \xi=N$.

System (2.1) is shown after normalization, using the scales $[L]=(c / \beta)^{1 / 2}$ and $[T]=(1 / c \beta)^{1 / 2}$ of length and time, respectively, and $h_{0}$ as the vertical scale, where $\xi=[u(x, y, t), v(x, y$, $t), \phi(x, y, t)]^{\mathrm{T}}$ is the state vector of the model and

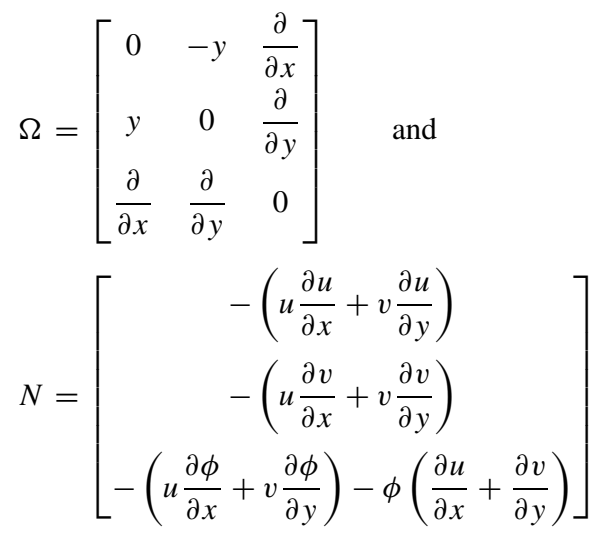

are the linear operator associated with the linearized equations and the non-linear term, respectively. Here, $u$ and $v$ are, respectively, the components of the two-dimensional velocity field on the $x$ (eastward) and $y$ (northward) directions; $\phi$ is the geopotential field of the free surface, $c=\sqrt{g h_{0}}$ is the phase speed of pure gravity waves, $h_{0}$ is the equivalent depth and $g$ is the effective gravity acceleration. The parameter $\beta=\mathrm{d} f / \mathrm{d} y$ is the Rossby parameter (where $f$ is the Coriolis parameter) and is assumed here as a constant and with its equatorial value.

System (2.1) is an appropriate model for describing atmospheric and oceanic motions with somewhat long time-scales and whose horizontal length-scales are relatively larger than their vertical ones, that is, for describing phenomena that do not depend crucially on temporal changes of the vertical density stratification. In the linear context, the shallow-water equations can also be regarded as the governing equations for the time evolution of the horizontal structure associated with a particular internal vertical mode of the upper-lidded primitive equations (Silva Dias et al., 1983). In this context, the equivalent depth $h_{0}$ is the separation constant and is obtained as an eigenvalue of the vertical structure equation. However, as far as the non-linear terms are restored this correspondence is no longer valid, because in this case the Galerkin projection of the three-dimensional primitive equations onto a particular vertical mode does not fall naturally into the non-linear shallow-water equations given by eqs. (2.1) and (2.2). As a consequence, caution is necessary in interpreting the results of this paper for the fully vertically stratified atmosphere, as shall be mentioned in Section 6 . 
Zonal periodicity in the $x$-direction and bounded solution as $|y|$ goes to infinity provide the boundary conditions for system (2.1). These boundary conditions define the eigenfunctions of the linear operator $\Omega$ as Hermite functions in the $y$-direction multiplied by trigonometric functions in the $x$-direction. Such eigenmodes form an orthogonal and complete set and are used as the basis functions in the expansion of $\xi$ in a series as in

$\xi(x, y, t)=\sum_{a} c_{a}(t) \xi_{a}$

where $c_{a}(t)$ are the spectral coefficients defined as the inner product between the state vector and a particular eigenfunction $\xi_{a}$. The subscript $a$ characterizes a particular expansion mode and is given by $a=(k, n, r)$, where $k$ is the zonal wavenumber, $n$ is the meridional mode, which distinguishes the meridional structure of the eigenfunctions; $r=1$ for Rossby waves (RW), $r=2$ for inertio-gravity waves propagating westward (WGW) and $r=3$ for inertio-gravity waves propagating eastward (EGW). The mixed Rossby-gravity waves (MRGW) correspond to the $n=0$ mode and are included in either $r=1$ (for $k>2^{-1 / 2}$ ) or $r$ $=2\left(\right.$ for $\left.k<2^{-1 / 2}\right)$ categories. The Kelvin wave is represented by $n=-1$ and $r=3$. The determination of the eigenfunctions of $\Omega$ and a complete discussion on the properties of such free linear wave solutions of the governing equations are found in Matsuno (1966).

For each expansion mode with parameters $\left(\omega_{a}, k_{a}, n_{a}, r_{a}\right)$, where $\omega_{a}$ are the eigenfrequencies associated with the eigenfunctions $\xi_{a}$, and the structure functions $\xi_{a}$, there is another mode, with parameters $\left(-\omega_{a},-k_{a}, n_{a}, r_{a}\right)$ and structure functions $-\xi_{a}^{*}$, where the superscript ${ }^{*}$ indicates the conjugate complex; the latter is denoted by $a^{*}$ and called as the conjugate mode of $a$. Two conjugate modes are mathematically independent, in the sense that the expansion (2.3) must consider both components of each conjugate pair $\left(a, a^{*}\right)$, but physically equivalent because the reality of the state variables $u, v$ and $\phi$ in eq. (2.3) implies that

$c_{a^{*}}=-c_{a}^{*}\left(\omega_{a^{*}}=-\omega_{a}, k_{a^{*}}=-k_{a}\right)$.

The expansion (2.3) is an exact solution of eq. (2.1) provided that the spectral coefficients satisfy the following system of ordinary differential equations

$\frac{\mathrm{d} c_{a}(t)}{\mathrm{d} t}-\mathrm{i} \omega_{a} c_{a}(t)=\sum_{b} \sum_{c} \sigma_{a}^{b c} c_{b}^{*}(t) c_{c}^{*}(t)$

In eq. (2.5) above, $\sigma_{a}^{b c}$ represent the coupling coefficients among three particular modes $a, b$ and $c$, and are given by

$$
\begin{aligned}
\sigma_{a}^{b c}= & \left\langle\left[ u_{b} \mathrm{i} k_{c} \xi_{c}+v_{b} \frac{\partial \xi_{c}}{\partial y}+(0,0,1)^{\mathrm{T}} \phi_{b}\right.\right. \\
& \left.\left.\times\left(\mathrm{i} k_{c} u_{c}+\frac{\partial v_{c}}{\partial y}\right)+C P\right] \cdot \xi_{a}\right\rangle \delta_{a b c} .
\end{aligned}
$$

In eq. (2.6) above, $\langle\cdot\rangle$ represents the inner product given by

$$
\begin{aligned}
\langle p(x, y) \cdot q(x, y)\rangle= & \int_{-L_{x}}^{L_{x}} \int_{-\infty}^{+\infty}\left(p_{1} q_{1}^{*}+p_{2} q_{2}^{*}+p_{3} q_{3}^{*}\right) \\
& \times \mathrm{d} y \mathrm{~d} x,
\end{aligned}
$$

where $p$ and $q$ are arbitrary three-dimensional vector fields satisfying the boundary conditions of model (2.1) and the subscripts 1, 2 and 3 refer to their scalar components; $L_{x}$ corresponds to the zonal period in dimensionless units and the superscript ' $T$ ' in eq. (2.6) refers to the transposition operation so that $(0,0,1)^{\mathrm{T}}$ corresponds to the unit vector in the vertical direction. The term $C P$ in eq. (2.6) means cyclical permutations between the superscripts $b c$ and, thus, the coupling coefficients $\sigma_{a}^{b c}$ are invariant under permutations of these indices. For a suitable normalization of the eigenfunctions $\xi_{a}$, the coupling coefficients are real. From eqs. (2.5) and (2.6) it can be noted that the non-linear interactions take place in triad configurations in which the coupling coefficients represent a measure of how far the product between two particular modes $\mathrm{b}$ and $\mathrm{c}$ can project onto another particular mode a. Thus, all information on the non-linearity of the model is contained in these coefficients. The coefficients $\delta_{a b c}$ represent the interaction condition for the triad $(a, b, c)$ and are given by

$\delta_{a b c}= \begin{cases}2 L_{x} \delta\left(k_{a}+k_{b}+k_{c}\right) & \text { if } n_{a}+n_{b}+n_{c}=\text { odd } \\ 0 & \text { otherwise }\end{cases}$

where the delta function in eq. (2.8a) results from the orthogonality of the trigonometric functions. It is well known (Phillips, 1960; Bretherton, 1964; Ripa, 1981, 1982, 1983a,b) that if the amplitude is small such that, to a first approximation, the evolution of the system is controlled by the linear part of eq. (2.5), only the interacting triads whose components satisfy the relation

$\omega_{a}+\omega_{b}+\omega_{c}=0$

contribute significantly to the non-linearity of the model. These triads whose components satisfy the interaction condition (2.8a) for $k_{a}+k_{b}+k_{c}=0$ and the resonance condition (2.9) are usually referred to as resonant triads.

This interesting phenomenon in non-linear wave-wave interactions was noticed by Phillips (1960) while discussing the role of small non-linear terms in the theory of ocean waves and has then been applied to a wide range of problems in physics. A rich and complete discussion on resonance in wave-wave interactions can be found in Bretherton (1964) in his analysis of a simple wave equation forced by a quadratic term. In the equatorial wave spectrum is a variety of resonant triads, as will be shown in Section 4. It is also important to mention that coupled resonant triads can also exist in the spectrum of the equatorial waves, i.e. resonant interactions involving a larger number of modes, as discussed later.

Therefore, knowledge of the possible resonant triads involving equatorial waves is of great importance for the tropical atmospheric dynamics, because, a priori, these triads determine the 
non-linear interactions in the spectrum of the equatorial waves. The next sections aim to analyze the time evolution of the mode energies in resonant triads. This analysis is no longer obvious and requires a further investigation on the internal dynamics of the model (2.1). This is exactly what shall be done in the next sections. Some constraints that the coupling coefficients must satisfy will be discussed in the next section. These are conditions that the coupling coefficients must satisfy as a consequence of the invariance of two particular integrals of motion, following the mathematical formalism of Ripa $(1982,1983 a)$. As will be shown, total energy and pseudo-momentum conservations lead to interesting properties that determine the time evolution of the modal amplitudes in a resonant triad and thus interesting implications for the dynamics of equatorial waves.

\section{Integral constraints}

System (2.1) is constrained by the existence of several integrals of motion. Two (and only two) of them have, to lowest order, a quadratic dependence in terms of the dependent variables of the model. The consequences of their conservation to the evolution of (2.1) in the phase space are more powerful than the consequences of the other conservation laws. These quadratic to lowest-order integrals of motion are the total energy and the total pseudo-momentum defined by their respective equations in conservative forms:

$$
\begin{aligned}
& \frac{\partial E}{\partial t}+\nabla \cdot\left[\vec{V}\left(E+\frac{1}{2} \phi^{2}\right)\right]=0 \\
& \frac{\partial P}{\partial t}+\nabla \cdot[\vec{V}(P+u)]+\frac{1}{2} \frac{\partial}{\partial x}\left[\phi^{2}-\left(u^{2}+v^{2}\right)\right]=0 .
\end{aligned}
$$

In the equations above, the superscript ' $\mathrm{T}$ ' corresponds to the transposition operation so that $\vec{V}=(u, v)^{\mathrm{T}}$ refers to the twodimensional velocity field vector. The variables $E$ and $P$ are, respectively, the energy and pseudo-momentum densities defined by

$E=\frac{1}{2}\left[\left(u^{2}+v^{2}+\phi^{2}\right)+\phi\left(u^{2}+v^{2}\right)\right]$

$P=\phi u-\frac{1+\phi}{2}(q-y)^{2}$

where $q$ is the potential vorticity and $y$ refers to the dimensionless Coriolis parameter.

The theoretical framework employed in this work follows the mathematical formalism of Ripa (1983a) and is also presented in Section 3 of Raupp and Silva Dias (2005). Thus, further details of this formalism will not be repeated here. The most important result for the purpose of the next sections is that, as a consequence of the conservation of total energy and pseudo-momentum, if a certain triad (a, b, c) satisfies the resonance condition (2.9) the coupling coefficients associated with this particular triad satisfy the relations

$\sigma_{a}^{b c}+\sigma_{b}^{a c}+\sigma_{c}^{a b}=0$

$s_{a} \sigma_{a}^{b c}+s_{b} \sigma_{b}^{a c}+s_{c} \sigma_{c}^{a b}=0$.

The parameter $s$ in eq. (3.2b) above is the slowness index (Ripa, 1981, 1982, 1983a,b), which is defined as the inverse of the zonal phase speed of the waves. Condition (3.2a) is a consequence of the conservation of total energy, whereas relation (3.2b) results from the pseudo-momentum conservation. These constraints have some interesting implications for the dynamics of the equatorial waves. The immediate consequence of eqs. (3.2a) and (3.2b) is that resonant interactions conserve the quadratic (lowest-order) part of total energy and pseudomomentum; the changes of $O\left(c^{3}\right)$ terms are owing to interactions by off-resonant triads. From eqs. (3.2a) and (3.2b) it can also be noted that, in a resonant triad, the component which has the coupling coefficient with the opposite sign of the other two (and, consequently, with the greatest absolute value) has the intermediate slowness. Thus, in this kind of interaction, the wave with the intermediate slowness always gains energy from (or releases energy to) the other two. Furthermore, using the definition of the slowness $s$ (inverse of the phase speed of the waves) and the interaction condition $\left(k_{a}+k_{b}+k_{c}=0\right)$, it is easy to show that the wave with the intermediate slowness in a certain resonant triad has the largest absolute frequency of the triad, i.e.

$\frac{\sigma_{a}^{b c}}{\omega_{a}}=\frac{\sigma_{b}^{a c}}{\omega_{b}}=\frac{\sigma_{c}^{a b}}{\omega_{c}}=\gamma_{a b c}$.

Therefore, in a resonant triad the wave with the largest absolute frequency is the most energetically active, that is, always gains energy from (or releases energy to) the other two components. Moreover, eq. (3.3) also shows that high-frequency modes in a resonant triad are energetically more active than the lowfrequency modes. As will be shown in the next sections, this constraint has an important implication not only in the determination of the possible resonant interactions but also in the time evolution of the wave energies in such interactions.

\section{Determination of the possible resonant triads among equatorial waves}

In this section we determine the possible resonant triads involving equatorial waves. The possible resonant triads were determined by the numerical calculation of the coupling coefficients in eq. (2.6) and by using the constraint (3.2a). For the numerical computation of the coupling coefficients in eq. (2.6) we used the Gauss-Hermite quadrature formula. It is important to mention that, because eq. (2.1) allows only a discrete number of normal modes, the resonance condition (2.9) cannot be exactly satisfied in practice. Thus, a certain tolerance $\Delta$ in eq. (3.2a) is needed in 
Table 1. Possible resonant triads involving equatorial waves for $h_{0}=250 \mathrm{~m}$. The table shows, from left to right, the triad components and their respective eigenfrequencies and coupling coefficients. The modes are characterized, from left to right, by the zonal wavenumber, the meridional mode and the wave type

\begin{tabular}{cccccccccc}
\hline & $a$ & $b$ & $c$ & $\omega_{a}$ & $\omega_{b}$ & $\omega_{c}$ & $\sigma_{a}^{b c}$ & $\sigma_{b}^{a c}$ & $\sigma_{c}^{a b}$ \\
\hline 1 & $0,-1, \mathrm{~K}$ & $1,1, \mathrm{WG}$ & $1,1, \mathrm{EG}$ & 0 & 1.71 & -1.78 & 0 & -0.1225 & 0.12765 \\
2 & $1,1, \mathrm{R}$ & $3,3, \mathrm{WG}$ & $4,3, \mathrm{EG}$ & 0.0758 & 2.687 & -2.86 & 0.00607 & 0.04377 & -0.0519 \\
3 & $5,1, \mathrm{R}$ & $1,3, \mathrm{EG}$ & $6,3, \mathrm{WG}$ & 0.2711 & -2.67 & 2.906 & 0.012614 & 0.054344 & -0.0631 \\
4 & $0,0, \mathrm{WG}$ & $4,1, \mathrm{WG}$ & $4,2, \mathrm{EG}$ & 1 & 1.83 & -2.49 & -0.028472 & -0.064398 & 0.0909 \\
5 & $0,1, \mathrm{R}$ & $5,2, \mathrm{R}$ & $5,0, \mathrm{M}$ & 0 & 0.183 & 0.577 & 0 & -0.07624 & 0.07588 \\
6 & $7,1, \mathrm{R}$ & $6,-1, \mathrm{~K}$ & $1,3, \mathrm{EG}$ & 0.292 & -1.39 & -2.67 & -0.00625 & -0.049643 & 0.05715 \\
7 & $5,-1, \mathrm{~K}$ & $2,1, \mathrm{EG}$ & $7,1, \mathrm{WG}$ & -1.156 & -1.86 & 2.21 & 0.01684 & 0.076963 & -0.0914 \\
8 & $1,2, \mathrm{EG}$ & $3,1, \mathrm{WG}$ & $4,0, \mathrm{M}$ & -2.27 & 1.756 & 0.64 & 0.09563 & -0.0816 & -0.0251 \\
9 & $5,0, \mathrm{M}$ & $1,2, \mathrm{WG}$ & $4,3, \mathrm{EG}$ & 0.577 & 2.225 & -2.86 & -0.0185 & -0.08019 & 0.10136 \\
10 & $2,-1, \mathrm{~K}$ & $7,0, \mathrm{M}$ & $5,2, \mathrm{EG}$ & -0.462 & 0.477 & -2.6 & 0.03389 & 0.007342 & -0.04 \\
\hline
\end{tabular}

order to find the possible resonant interactions. The number of resonant triads found by condition (3.2a) depends on the tolerance and, through the fundamental wavenumber, on the equivalent depth $h_{0}$. However, although the choice of the tolerance $\Delta$ and the equivalent height $h_{0}$ influence the size of the resonance spectrum, it was found that the resonant triads are highly self-consistent, i.e. exhibit a remarkably similar composition irrespective of $h_{0}$ and the choice of $\Delta$. Therefore, for an illustrative purpose, only unique values of $h_{0}(250 \mathrm{~m})$ and $\Delta\left(10^{-2}\right)$ are discussed here (Table 1). This value of $h_{0}$ is associated with the dominant vertical mode for the atmospheric flow in the tropical region (Silva Dias and Bonatti, 1985), because it corresponds to the mostly excited vertical mode by the typical heating resultant from the organized moist convection in the tropics (DeMaria, 1985). Table 1 shows the mode components of each triad and their correspondent eigenfrequencies and coupling coefficients. It is important to state that Table 1 presents only some examples of the main kinds of resonant triads found. Obviously, there are more triads that are not shown in Table 1 but these triads have the same kind of waves as those shown in Table 1.

Table 1 shows that, in general, the resonant triads obtained involve all the equatorial wave types. They are strongly dominated by triads with two high-frequency modes (Kelvin or inertiogravity) and one low-frequency mode (Rossby or mixed Rossbygravity). Some strictly low-frequency (case 5 of Table 1) and strictly high-frequency triads (cases 4 and 7) also exist, but no triads involving solely the inertio-gravity modes, satisfying condition (3.2a), could be found.

From Table 1 it can be noted that the coupling coefficients in a resonant triad involving equatorial waves are indeed proportional to the individual absolute frequencies of the wave components. As a consequence, in a resonant interaction involving two inertio-gravity modes and one Rossby mode the coupling coefficient associated with the Rossby mode is much smaller in absolute value than the coupling coefficients associated with the inertio-gravity modes. As a result, this kind of interaction is characterized by a catalytic energy exchange between the two high-frequency modes, while the Rossby wave energy remains almost unchanged in time, as will be shown in the next section. Especially, in resonant triads containing the zonally symmetric Rossby or Kelvin modes, as a consequence of the zero-frequency of these modes, their coupling coefficients are zero and thus their energy remains completely unaltered in a resonant interaction.

Loesch and Deininger (1979) determined the resonant triads among equatorial waves using the conditions (2.8a) and (2.9), together with the dispersion relation, following Longuet-Higgins and Gill (1967). In general, the resonant interactions found by Loesch and Deininger are in agreement with those obtained here.

\section{Dynamics of resonant interactions among equatorial waves}

Possible resonant interactions involving equatorial waves were discussed in the previous section. As, in a first approximation, only these triads have a significant role on the non-linear evolution of eq. (2.5), besides the knowledge of the possible resonant triads it is also interesting to analyze the triad dynamics. The dynamics of the resonant three-wave problem is discussed in Section 5.1, i.e. system (2.5) is truncated to consider only three waves satisfying the conditions (2.8a) for $k_{a}+k_{b}+k_{c}=0$ and (3.2a). The problem of two resonant triads coupled by a single mode is discussed in Section 5.2.

\subsection{Resonant three-wave problem}

The resonant three-wave problem has the following formulation in the phase space:

$$
\begin{aligned}
& \frac{\mathrm{d} c_{1}}{\mathrm{~d} t}-\mathrm{i} \omega_{1} c_{1}=\sigma_{1}^{23} c_{2}^{*} c_{3}^{*} \\
& \frac{\mathrm{d} c_{2}}{\mathrm{~d} t}-\mathrm{i} \omega_{2} c_{2}=\sigma_{2}^{13} c_{1}^{*} c_{3}^{*} \\
& \frac{\mathrm{d} c_{3}}{\mathrm{~d} t}-\mathrm{i} \omega_{3} c_{3}=\sigma_{3}^{12} c_{1}^{*} c_{2}^{*} .
\end{aligned}
$$




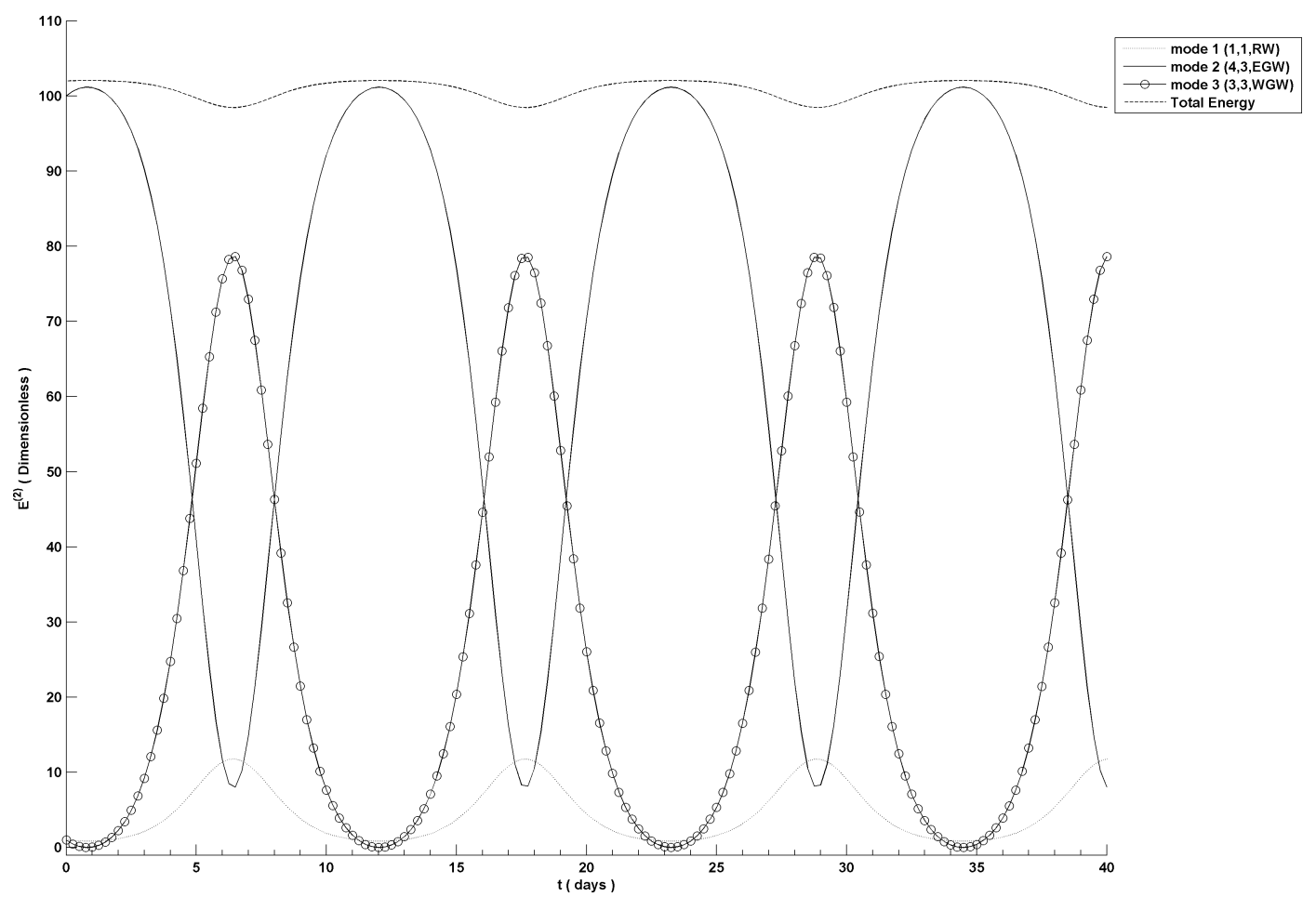

Fig. 1. Time evolution of the modal quadratic energies associated with the solution of the resonant three-wave problem for the triad composed of a Rossby wave (RW) with $k=1$ and $n=1$ (mode 1), an inertio-gravity wave propagating eastward (EGW) with $k=4$ and $n=3$ (mode 2 ) and an inertio-gravity wave propagating westward (WGW) with $k=3$ and $n=3$ (mode 3 ). The total quadratic energy given by the sum of the energy of the individual components is also displayed. The initial amplitudes of the triad components are given by $A_{1}=A_{3}=1$ and $A_{2}=10$.

The above system is in Hamiltonian form, with $\left(c_{j}, c_{j}^{*}\right), j=1,2$ or 3 , being the canonically conjugate variables. The Hamiltonian form of eqs. (5.1) is

$\frac{\mathrm{d} c_{j}}{\mathrm{~d} t}=\mathrm{i} \omega_{j} \frac{\partial H}{\partial c_{j}^{*}}, \quad j=1,2$ or 3

with the Hamiltonian $H$ given by

$H=\sum_{j} \gamma_{123} \operatorname{Im}\left(c_{1} c_{2} c_{3}\right)=$ constant

where the coefficient $\gamma_{123}$ in eq. (5.3) is given by eq. (3.3). Thus, the dynamical system (5.1) has three degrees of freedom and, at least, three integrals of motion: the Hamiltonian $H, E^{(2)}$ and $P^{(2)}$ (which refer, respectively, to the quadratic part of total energy and pseudo-momentum). Therefore, the three-wave problem is integrable. General solution of this problem can be obtained in terms of Jacobian elliptic functions as in Domaracki and Loesch (1977) and Ripa (1981).

Although the dynamics of the resonant three-wave problem (5.1) is well known (Domaracki and Loesch, 1977; Ripa, 1981, and others), we show here an example of numerical integration of this problem in order to fix ideas and to discuss the implications of this triad dynamics for some aspects of the tropical atmospheric circulation. We believe that the original contribution of this work for the dynamics of resonant triads stems from the results of the five-wave dynamics shown in Section 5.2. The numerical integration scheme of eq. (5.1) assumes that the non-linear terms in the right-hand side of eq. (5.1) are constant forcings during a very short time $2 \Delta t$ so that, given the coefficients $c_{j}, j=1,2$, and 3 , at $t-\Delta t$ and $t$, an analytic solution can easily be obtained at $t+\Delta t$. In this scheme, the non-linear terms are evaluated at the central level $t$. The initial condition used in the numerical simulations is given by

$c_{j}(0)=A_{j} \mathrm{e}^{\mathrm{i} \lambda_{j}}$

where $j=1,2,3$. The initial phases $\lambda j$ are chosen such that $\sum_{j} \lambda_{j}=\pi / 2$, in order to obtain the maximum energy exchange (Domaracki and Loesch, 1977; Loesch and Deininger, 1979).

The example of the numerical integration of the problem (5.1) is displayed in Fig. 1 for the triad constituted of a RW with $k=$ 1 and $n=1$ (mode 1), an EGW with $k=4$ and $n=3$ (mode 2) and a WGW with $k=3$ and $n=3$ (mode 3 ). This triad refers to case 2 of Table 1 . In this triad, mode 2 has the largest absolute frequency and, thus, the maximum coupling coefficient in absolute value. Therefore, this inertio-gravity mode is the most energetically active of the triad, i.e. corresponds to the wave which supplies energy to the other components or gains energy from the other two modes. In this integration, the initial condition is almost all projected onto mode 2 and only a small fraction is projected onto the other components $\left(A_{1}=A_{3}=1\right.$ and $A_{2}=$ 


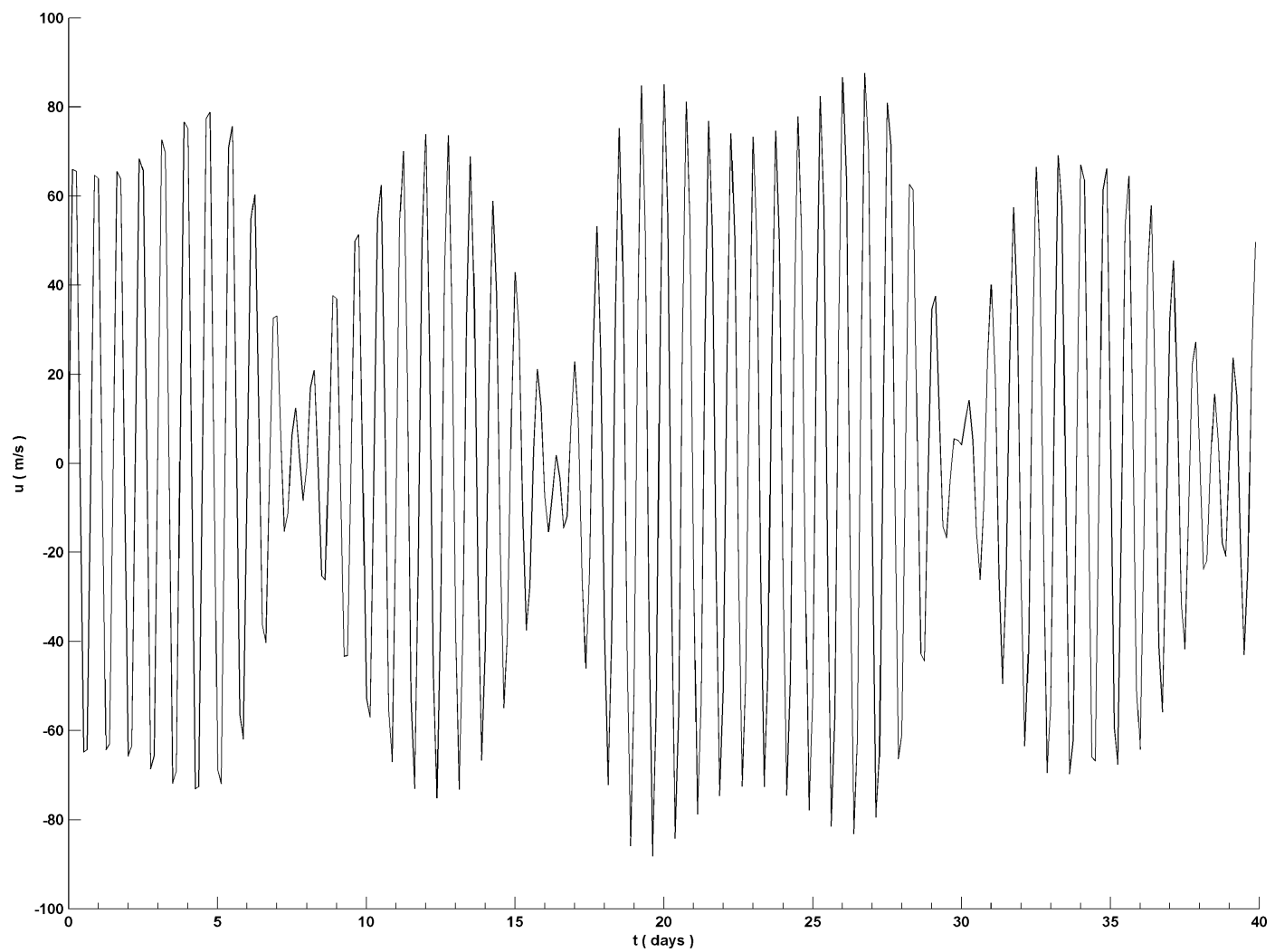

Fig. 2. Time evolution of the zonal component of the velocity field at a certain point of the domain correspondent to the numerical solution of Fig. 1.

10). Thus, this case is a typical problem of wave instability. As resonant interactions conserve the quadratic part of total energy, the energy of a particular resonant-triad component is simply given by the square of its amplitude.

Figure 1 shows that, when the initial condition is almost totally projected onto the highest-frequency mode, there is a parametric instability of this mode (mode 2), which supplies energy to the components 1 and 3 according to conditions (3.2a) and (3.2b). Nevertheless, after a certain time, mode 2 energy becomes so small that the direction of the energy transfer is reversed, and mode 2 starts to receive energy from the components 1 and 3 . As time evolves, the cycle repeats itself and the energy of each component continues to alternate periodically. Thus, the solution of the resonant three-wave problem is always periodic in time, that is, the triad components exchange energy in a periodic manner while the total energy $E^{(2)}$ remains constant. Some oscillations in the total energy $E^{(2)}$ are observed in Fig. 1 and are due to the fact that the resonance condition is not exactly satisfied, as already discussed.

Another important feature on the triad dynamics involving equatorial waves is that the variation of the RW energy is very small compared to the variation of the gravity wave energies, as expected from eq. (2.5) and from the values of the coupling coefficients (see case 2 of Table 1). Thus, the dynamics of a resonant triad like that of case 2 of Table 1 is characterized by a catalytic energy exchange between the two gravity modes, while the RW energy remains almost unchanged during the process. Therefore, in a resonant triad with two high-frequency modes and one low-frequency mode, the low-frequency mode only acts as a catalytic component. This property implies that the magnitude of the energy modulations is dependent on the distribution of the initial energy among the triad components, as also pointed out by Domaracki and Loesch (1977). In fact, if the initial condition is mostly projected onto mode 1 , the energy exchanges are strongly inhibited (figure not shown).

Another interesting feature regarding the solution of the resonant three-wave problem is that the period of the energy modulations is dependent upon the initial amplitudes but is always finite. Furthermore, the higher the values of the initial amplitudes, the shorter the period of the amplitude modulations (Domaracki and Loesch, 1977).

The important point to be analyzed here refers to the implication of the energy exchanges among resonant triad components for the solution in physical space. Fig. 2 shows the time evolution of the zonal wind field at a certain point of the domain associated with the numerical solution of Fig. 1. The zonal wind field in Fig. 2 was obtained by the expansion (2.3) considering only the three modes of the resonant triad.

The time evolution of the zonal wind field illustrated in Fig. 2 displays the existence of local high-frequency oscillations with a 
period shorter than $1 \mathrm{~d}$. These high-frequency local oscillations are associated with the phase propagation of the two inertiogravity modes of the second triad of Table 1 . In fact, for $h_{0}=$ $250 \mathrm{~m}\left(c=50 \mathrm{~m} \mathrm{~s}^{-1}\right)$, the eastward inertio-gravity wave labeled as mode 2 in Fig. 1, which is characterized by zonal wavenumber 4 and meridional mode 3 , has a time frequency corresponding to a period of the order of $16 \mathrm{~h}$. This short period of the local oscillations may be somewhat unrealistic in the sense that it might not be observed in a more realistic large-scale atmospheric model, which includes a parametrization of the feedback between large-scale dynamics and moist convection. When the interaction between the equatorial wave dynamics and moist convection is taken into account, there is a reduction in the effective equivalent depth of the shallow-water equations and, as a result, there is a reduction in the time frequency of the eigenmodes. In fact, if one assumes that the convective forcing is proportional to divergence, in a first approximation, the divergent term in the continuity equation of model (2.1) appears multiplied by an effective equivalent depth that is smaller than the true equivalent depth. The stronger the convection related to the divergence, the smaller the effective equivalent depth. Therefore, the period of high-frequency oscillations observed in Fig. 2 may reflect the limitation of the model adopted in the present work in not taking into account the interaction between equatorial waves and moist convection. Nevertheless, these high-frequency local oscillations may be, a priori, unimportant for the results presented here, because the focus of the present study is the non-linear interactions. Consequently, we have adopted a phenomenological model with the goal of analyzing only the dynamics of the interaction among equatorial waves through the non-linear terms into the governing equations. The role of reducing the equivalent depth of system (2.1), which implies the incorporation of the interaction between convection and dynamics in the model, on the non-linear dynamics explored here would be an interesting subject for a future work.

The lower-frequency modulation in the amplitude of these local oscillations is resultant from the energy modulations associated with the interactions among the wave modes in the resonant triad. This can be concluded by comparing Figs. 2 and 1. The time when the magnitude of the zonal wind is maximal corresponds exactly to the time when the energy of the EGW (mode 2) peaks. On the other hand, when the WGW energy is maximal (and the EGW energy is minimal) corresponds to the time when the amplitude of the zonal wind oscillations is minimal. This is in agreement with the spatial structure of these equatorial waves, because the zonal kinetic energy of the inertio-gravity modes increases as zonal wavenumber increases (shorter waves) (Silva Dias and Schubert, 1979).

Thus, the periodic exchanges of energy among waves constituting a resonant triad imply in periodic changes of regime in the physical space solution. Such changes of regime, in turn, occur on a longer time-scale than the period of the local highfrequency oscillations. This periodic change of regime in the solution in physical space due to the internal dynamics of the model is known as vacillation (Lorentz, 1963). Other mechanisms are also known to induce vacillating solutions, such as wave-zonal flow interactions (Pedlosky, 1977; Boville, 1980) and interference between linear waves with the same wavenumber and different phase speeds (Lindzen et al., 1982). As our results point out, non-linear interactions among waves constituting a resonant triad can also lead to vacillations. The high peak magnitudes of the order of $100 \mathrm{~m} \mathrm{~s}^{-1}$ observed in Fig. 2, which are somewhat unrealistic, are resultant from the unrealistically high value of the mode 2 amplitude set in Fig. 1. We have selected this unrealistic initial wave amplitude in order to more clearly observe the features associated with the dynamics of resonant triads. Exaggerating the high values of the initial amplitudes as done in Fig. 1 makes the dynamical features of the resonant triads, such as the parametric instability of the highest frequency mode, more clearly noticeable. However, it is important to mention that, in a qualitative sense, the results shown in Figs. 1 and 2 are not affected by the unrealistic initial amplitude set in Fig. 1. That is, had we selected lower values for the initial amplitudes in Fig. 1, the behavior of the solution would have been the same as observed in Figs 1 and 2, but the value of the period of the low-frequency amplitude modulation (vacillation) resultant from the energy exchanges would have been different (longer). The only caution that we should have is regarding the conclusions drawn from the periods of the vacillations observed in Fig. 2, as shall be discussed later.

Therefore, the dependence of the period and amplitude of the energy modulation of the modes constituting resonant triads previously discussed have an important implication for the numerical weather prediction. This sensitivity demonstrates that the period of the vacillation cycle of the flow is dependent on the magnitude of the initial field and the way in which this initial field is projected onto the modes that represent the atmospheric flow. The way in which a given state of the atmosphere (which can be used as initial condition in a weather prediction) projects onto the modes, in turn, is very dependent on the manner in which a numerical model represents certain physical process in the atmosphere. For example, gravity waves are directly linked to moist convection. As a consequence, the projection of the initial field onto gravity modes, and consequently the vacillation cycle of the atmospheric flow, can be sensitive to the representation of such moist process in the numerical model, which is highly dependent on the convective parametrization.

\subsection{Problem of two resonant triads coupled by one mode}

In the previous subsection we discussed the dynamics of the energy exchanges among equatorial waves constituting a single resonant triad. Nonetheless, in a more realistic situation, a unique equatorial wave can participate in several resonantly triadic interactions. Thus, in the equatorial wave spectrum are numerous coupled resonant triads, allowing the spectral energy 
redistribution. This shows that resonant interactions can have an important role in the generation of turbulence, making the analysis of the dynamics of resonant triads very useful in understanding the transition to turbulent regimes in dynamical systems. Loesch and Deininger (1979) investigated the effectiveness with which resonant interactions among equatorial waves can redistribute energy generated at selective scales. They analyzed the dynamics of closed systems composed of several resonant triads and pointed out that the energy transference throughout the system is very dependent on the energy projected onto the maximum-frequency modes of individual triads. Here, we explore the dynamics of a more simplified system constituted by only two resonant triads coupled by a unique mode in order to further explore the energy transference between different triads as well as the implications of this process for the tropical atmospheric climate.

Let us consider mode 1 as the mode which couples the two triads. Thus, considering the triads $(1,2,3)$ and $(1,4,5)$, this problem is formulated in terms of the spectral amplitudes as follows:

$$
\begin{aligned}
& \frac{\mathrm{d} c_{1}}{\mathrm{~d} t}-\mathrm{i} \omega_{1} c_{1}=\sigma_{1}^{23} c_{2}^{*} c_{3}^{*}+\sigma_{1}^{45} c_{4}^{*} c_{5}^{*} \\
& \frac{\mathrm{d} c_{2}}{\mathrm{~d} t}-\mathrm{i} \omega_{2} c_{2}=\sigma_{2}^{13} c_{1}^{*} c_{3}^{*} \\
& \frac{\mathrm{d} c_{3}}{\mathrm{~d} t}-\mathrm{i} \omega_{3} c_{3}=\sigma_{3}^{12} c_{1}^{*} c_{2}^{*} \\
& \frac{\mathrm{d} c_{4}}{\mathrm{~d} t}-\mathrm{i} \omega_{4} c_{4}=\sigma_{4}^{15} c_{1}^{*} c_{5}^{*} \\
& \frac{\mathrm{d} c_{5}}{\mathrm{~d} t}-\mathrm{i} \omega_{5} c_{5}=\sigma_{5}^{14} c_{1}^{*} c_{4}^{*} .
\end{aligned}
$$

Problem (5.5) has the same Hamiltonian form given by eq. (5.2) with the Hamiltonian given by

$H=\sum_{a b c} \gamma_{a b c} \operatorname{Im}\left(c_{a} c_{b} c_{c}\right)=$ constant

where the subscripts $a, b$ and $c$ range from 1 to 5 . However, unlike the three-wave problem, nothing can be said a priori on the integrability of the five-wave system. As in the three-wave problem, the initial condition is given by eq. (5.4) but with $j=$ $1,2,3,4,5$ and the initial phases are chosen in order to obtain the maximum energy exchange.

Examples of numerical solutions of eq. (5.5) are shown in Fig. 3 for the case in which the modes 1, 2, 3, 4 and 5 are given by, respectively: an MRGW with $k=5$, an MRGW with $k=1$, an EGW with $k=4$ and $n=1$, a WGW with $k=1$ and $n=2$ and an EGW with $k=4$ and $n=3$. Thus, it can be noted that, in this case, the mode that couples the triads is the lowest-frequency mode in the two triads, i.e. the energetically less active member of the two triads.

Figure $3 \mathrm{a}$ shows the time evolution of the mode energies for the case in which the initial condition is almost projected onto mode 5 , which corresponds to the highest-frequency mode of the second triad. As in the three-wave problem, when the initial condition is almost projected onto the highest-frequency mode, there is an initial parametric instability followed by an inversion of the energy transference and a consequent periodic evolution of the energy with time, as shown in Fig. 3a. It is interesting to observe that modes 2 and 3 of the first triad are not significantly excited, that is, their energy is kept suppressed in time. Thus, the solution converges to the three-wave problem and there is no an intertriad energy distribution. The same occurs when the initial condition is equally projected onto the two stable waves of a particular triad, as can be noted in Fig. 3b. In this case, as in Fig. 3a, there is a catalytic energy exchange between the two gravity waves (highest-frequency modes) of the second triad, whereas the MRGW with $k=5$ (which is in the low-frequency category) remains essentially energetically unaltered.

Figure $3 c$ illustrates the case in which all five waves have the same amplitude, i.e. for a 'white noise' initial perturbation. In this case, as observed in Fig. 3c, there are catalytic energy exchanges involving the two highest-frequency modes of each triad. An energy exchange among components of different triads does not occur. In other words, there is no effective intertriad coupling. An interesting feature regarding the solution of system (5.5) occurs when the energy associated with the initial perturbation is equally distributed between two modes of different triads. In this case, as can be seen in Fig. $3 \mathrm{~d}$ for the case in which the initial perturbation is essentially projected onto components 3 and 5 , there are the same catalytic and periodic energy exchanges among modes of the same triad. However, it is clear in the time evolution of the energy of modes 2 and 3 the alternation of large and small energy peaks, suggesting that the period of the energy exchange between these wave components has doubled. This period-doubling seems to be a result of the larger number of degrees of freedom of system (5.5) in comparison to the three-wave problem. The higher the number of triads included in the three-wave problem (5.1), the larger the degrees of freedom of the resulting system, and thus the more irregular the time evolution of the mode energies, tending to a chaotic behavior as the number of degrees of freedom becomes higher. Therefore, the period-doubling of the energy modulation noted in Fig. 3d seems to be an indication of the non-integrability of system (5.5) and, consequently, a possible path to chaos, because the period-doubling rout to chaos is a very well-known example of bifurcation in dynamical systems (Ott, 1993).

Nevertheless, despite the period-doubling of the energy modulations indicating a possible path to chaos as more triads of waves are included to the problem (5.1), the time evolution of the modal energies shown in Fig. 3d also does not display a significant coupling between components of different triads. This absence of an effective intertriad coupling can be due to the fact that the mode that couples the triads is exactly the lowest-frequency mode of the two triads (i.e. the less energetically active member of the system).

In fact, Fig. 4 shows that when the mode coupling the two triads is exactly the highest-frequency mode of the two triads, 
(a)

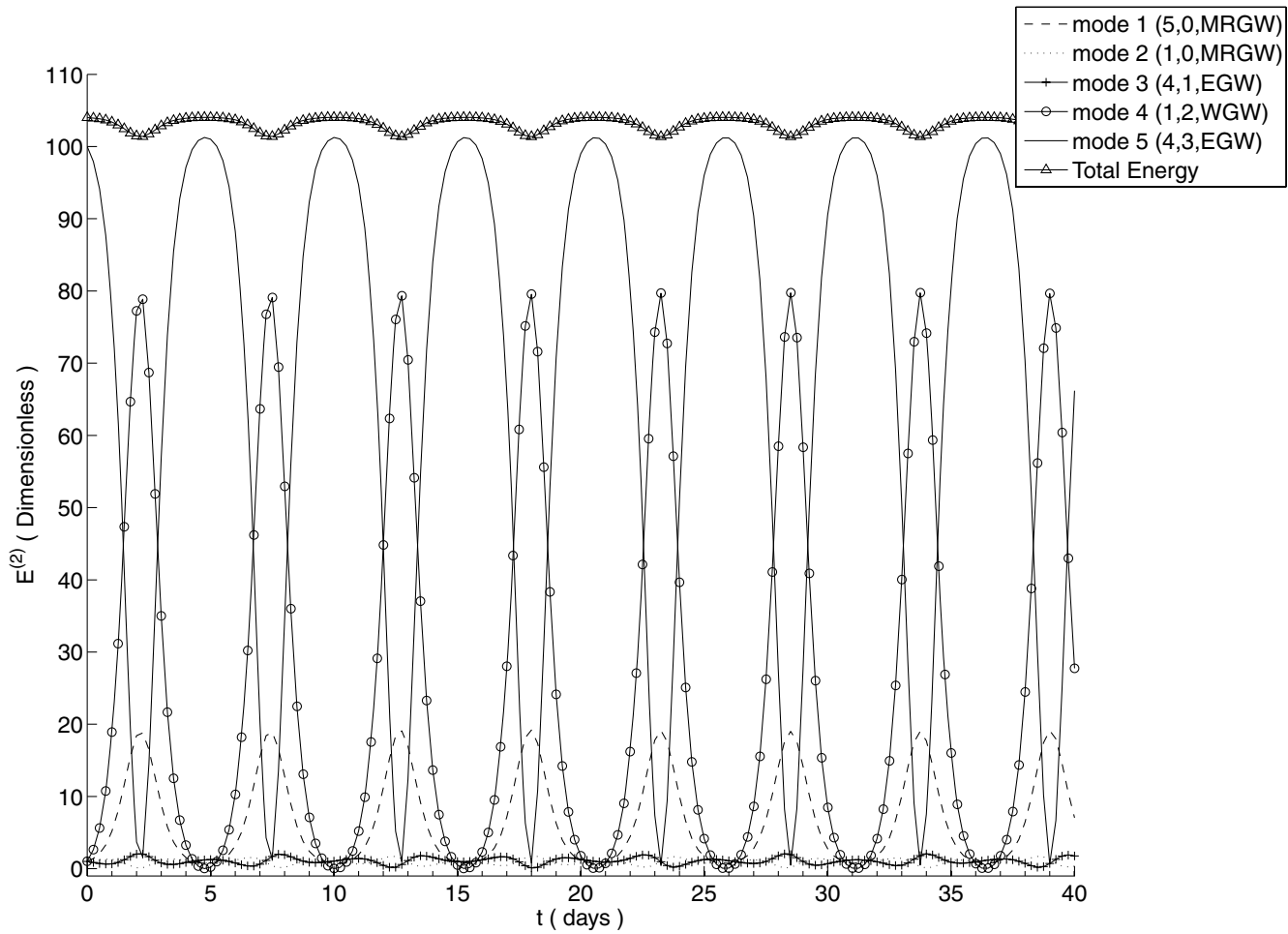

(b)

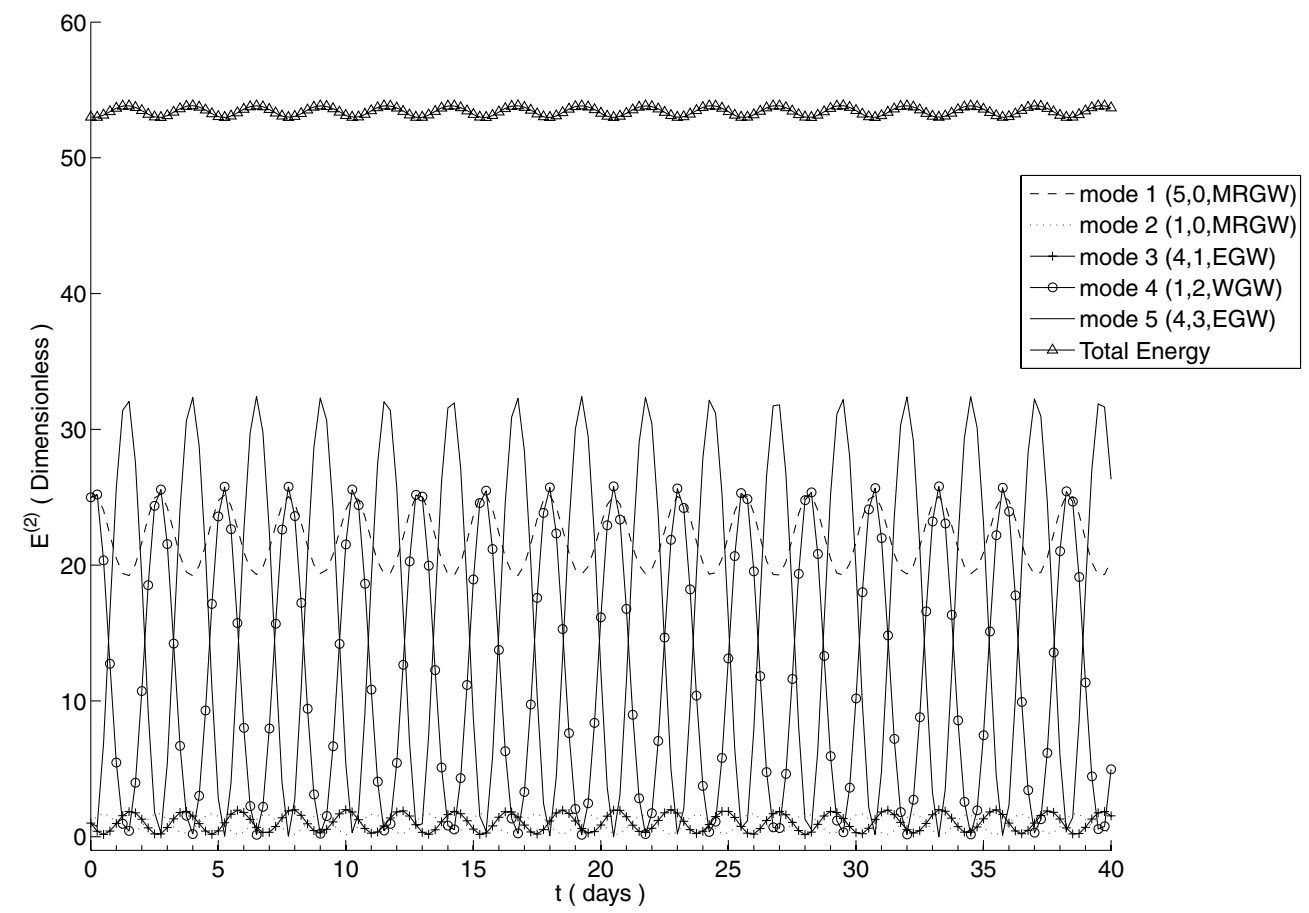

Fig. 3. Time evolution of the modal quadratic energies associated with the solution of problem (5.5) composed of an MRGW with $k=5$ (mode 1), an MRGW with $k=1$ (mode 2), an EGW with $k=4$ and $n=1$ (mode 3), an WGW with $k=1$ and $n=2$ (mode 4) and an EGW with $k=4$ and $n=3$ (mode 5). The total quadratic energy given by the sum of the energy of the individual components is also displayed. The initial amplitudes of the modes are given by: (a) $A_{1}=A_{2}=A_{3}=A_{4}=1$ and $A_{5}=10$; (b) $A_{1}=A_{4}=5$ and $A_{2}=A_{3}=A_{5}=1$; (c) $A_{1}=A_{2}=A_{3}=A_{4}=A_{5}=1$; (d) $A_{1}=A_{2}=A_{4}=1$ and $A_{3}=A_{5}=5$. 
(c)

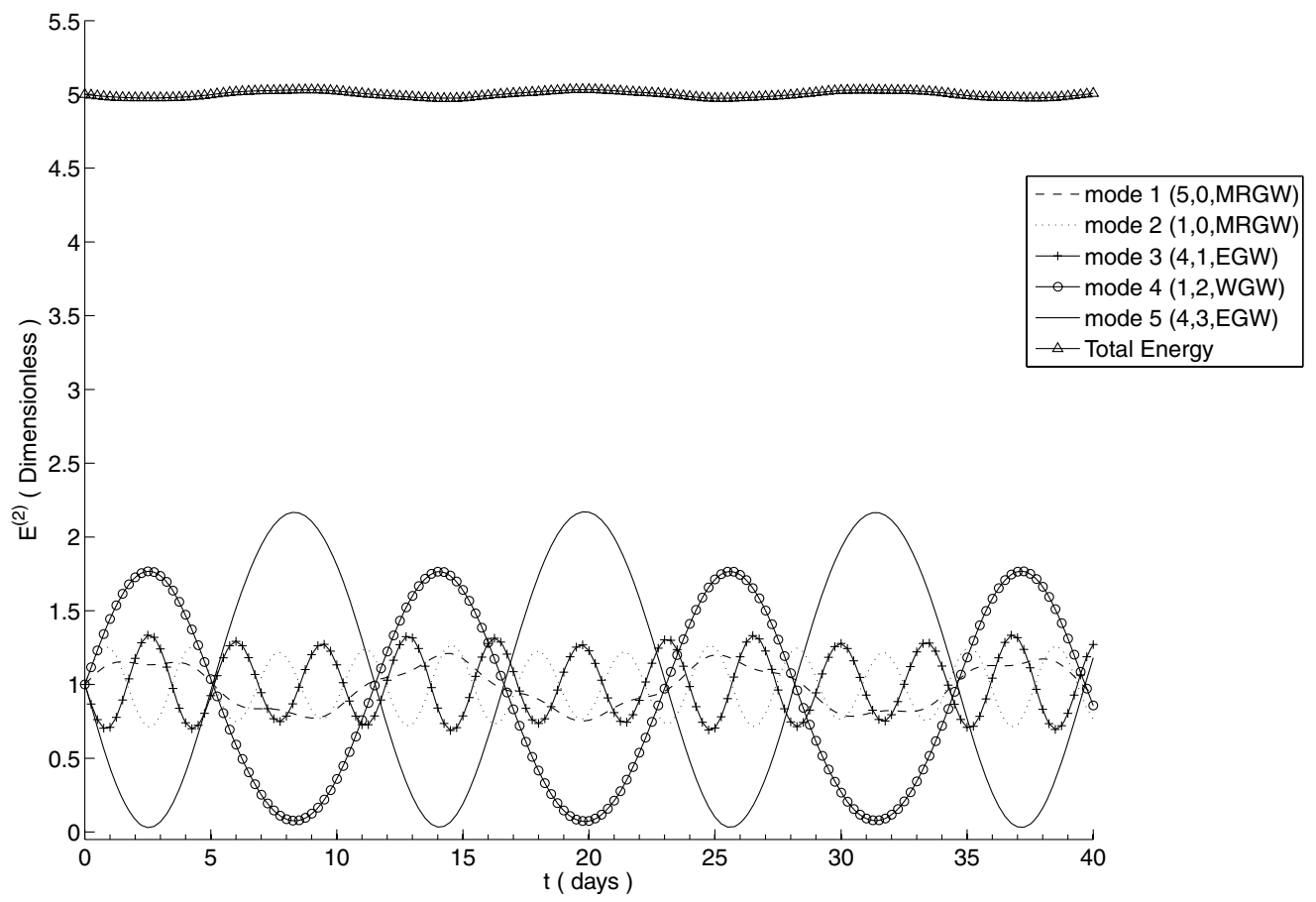

(d)

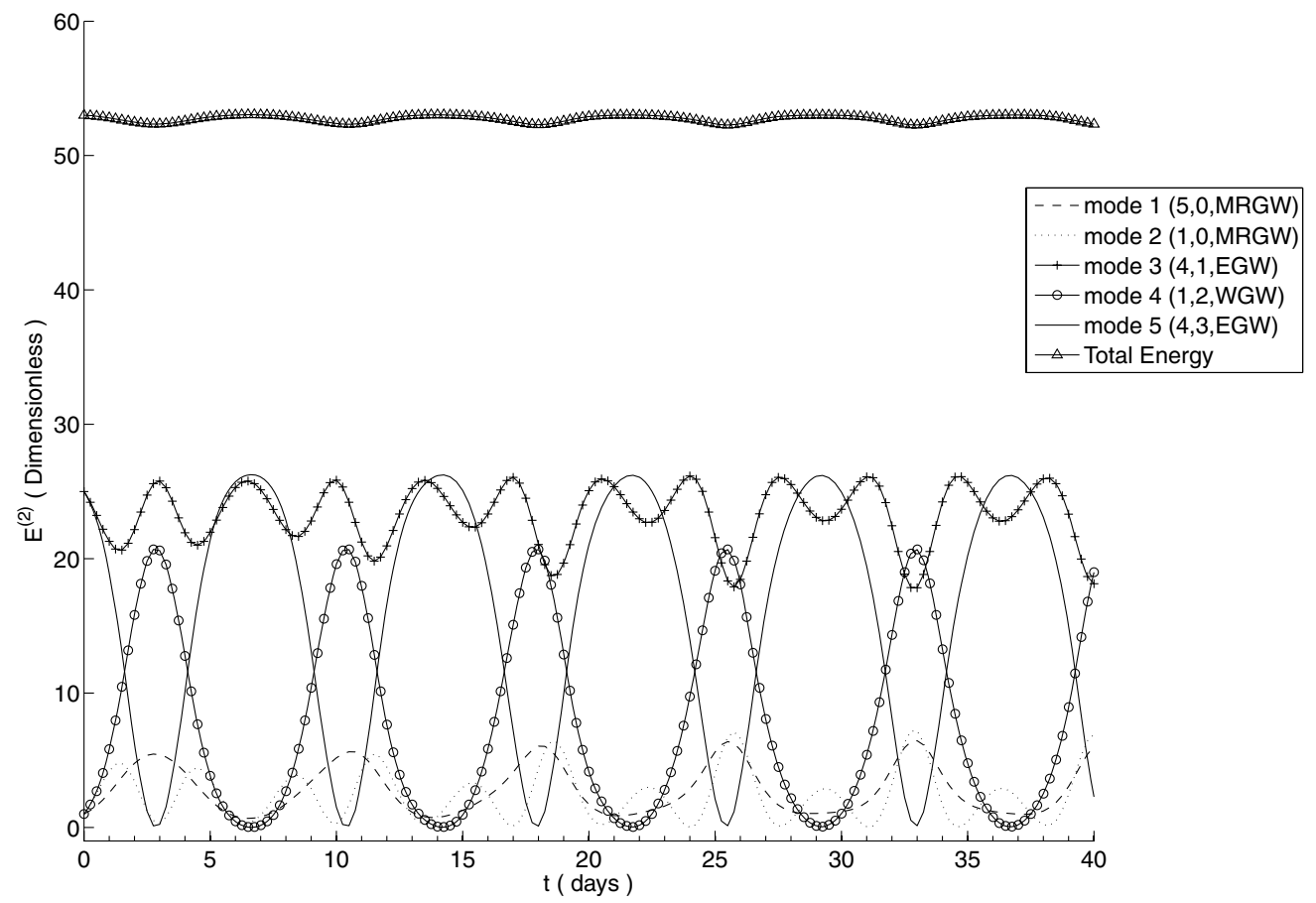

Fig. 3. (cont'd).

a significant intertriad coupling takes place. Figure 4 shows the time evolution of the mode energies associated with the problem (5.5) where the constituting modes are: an EGW with $k=6$ and $n$ $=2($ mode 1$)$; a WGW with $k=6$ and $n=1$ (mode 2$)$; a zonally symmetric MRGW $(k=0)$; a WGW with $k=1$ and $n=3$ (mode 4) and an MRGW with $k=7$ (mode 5). It is important to point out that, in this case, the mode which couples the two triads is exactly the highest-frequency mode of the problem. Figure 4a shows the 
(a)

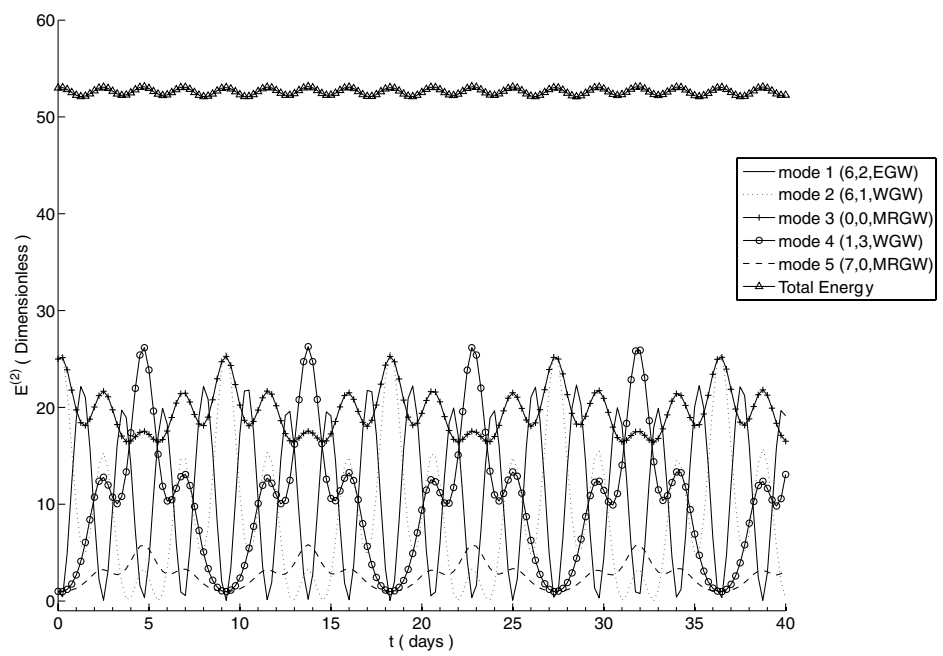

(b)

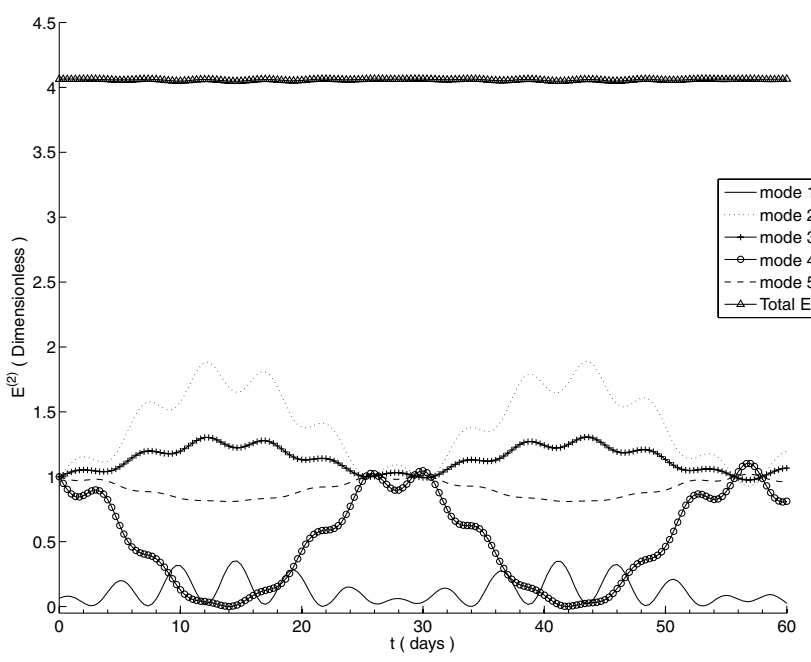

Fig. 4. Similar to Fig. 3, but for the components given by an EGW with $k=6$ and $n=2$ (mode 1$)$, an WGW with $k=6$ and $n=1$ (mode 2), an MRGW with $k=0$ (mode 3 ), an WGW with $k=1$ and $n=3$ (mode 4) and an MRGW with $k=7$ (mode 5). The initial amplitudes in this case are given by: (a) $A_{2}=A_{3}=5$ and $A_{1}=A_{4}=$ $A_{5}=1$ and (b) $A_{1}=0.25$ and $A_{2}=A_{3}=$ $A_{4}=A_{5}=1$.

example in which the initial condition is essentially projected onto the two stable components of the first triad, the modes 2 and 3. Unlike the previous case (Fig. 3b), a significant redistribution of energy occurs, as can be noted by the excitation of mode 4 . It is interesting to note that, besides the energy exchanges among components of the same resonant triad, a noteworthy energy exchange between modes of different triads occurs, as can be seen by the noticeable exchange of energy between modes 4 and 2. Another important feature to be noticed in Fig. 4a is that the period associated with the intertriad energy exchanges is longer than that associated with the intratriad interactions. This longer time-scale of the intertriad energy exchanges is because of the fact that the intertriad coupling is indirect, unlike the intratriad one, as can be seen by eq. (5.5). In order for the mode 2 to supply energy to mode 4 , for example, it has to supply energy to mode 1 at first.

The same intertriad energy exchange observed in Fig. 4a can be more clearly noted in Fig. 4b, which corresponds to the case in which the initial condition is given by a 'white noise' initial perturbation, except that mode 1 has one-quarter of the amplitude of the other modes $\left(A_{1}=0.25 ; A_{2}=A_{3}=A_{4}=\right.$ $\left.A_{5}=1\right)$. It is important to observe that the longest period of the energy modulation associated with the intertriad interactions in this case is of order of $30 \mathrm{~d}$ (i.e. even longer than in Fig. 4a).

Figure 5 shows the time evolution of $u$ (zonal component of the wind field) at a certain point of the domain correspondent to the solution of the problem (5.5). Figure 5a corresponds to the numerical solution of Fig. 4b, while Fig. $5 \mathrm{~b}$ refers to the same numerical solution as Fig. 5a, but with component 1 having four times the amplitude of the other components $\left(A_{1}=4 ; A_{2}=A_{3}=\right.$ $A_{4}=A_{5}=1$ ). Similarly to the three-wave problem, the $u$ field in Fig. 5 is obtained by the spectral expansion (2.3) truncated in order to consider the five components of problem (5.5). One can note in Fig. 5a three distinct oscillations: a high-frequency oscillation associated with the phase propagation of the 
(a)

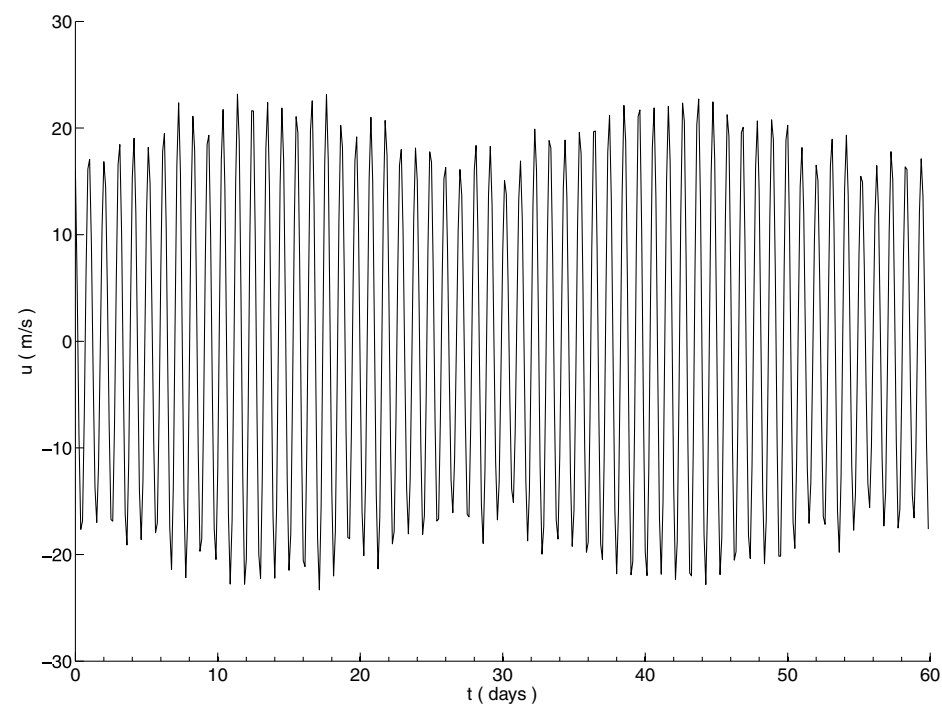

(b)

Fig. 5. Time evolution of the zonal component of the velocity field at a certain point of the domain correspondent to the solution of problem (5.5) with the same mode components as Fig. 4 for (a) $A_{1}=$ 0.25 and $A_{2}=A_{3}=A_{4}=A_{5}=1$ and (b) $A_{1}=4$ and $A_{2}=A_{3}=A_{4}=A_{5}=1$.

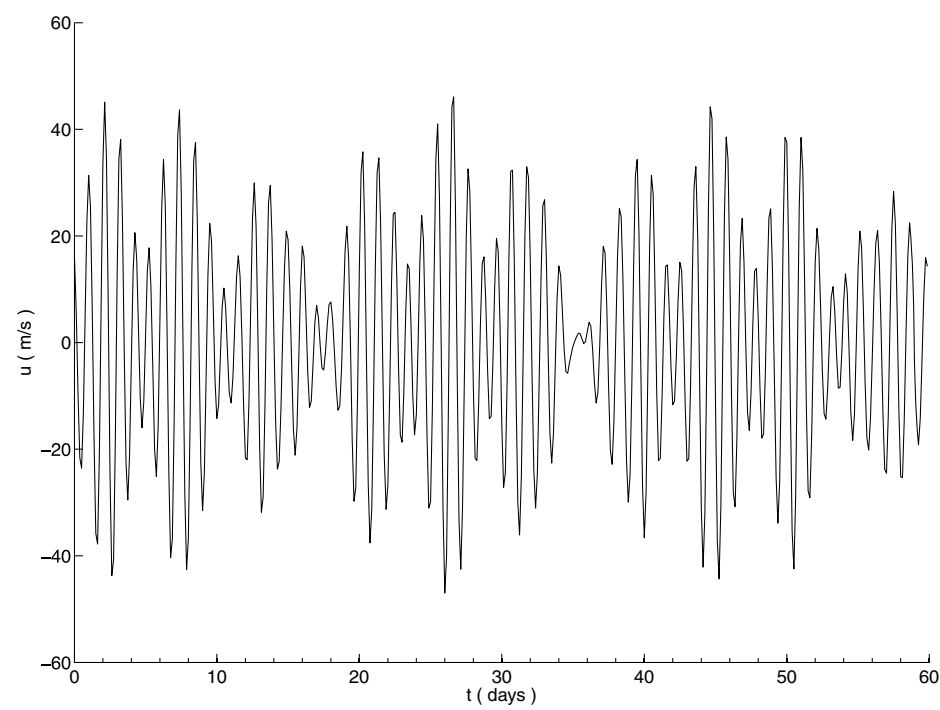

inertio-gravity waves of the two triads; a modulation with longer period (5-8d), which can be noticed by the alternation of smaller and larger peaks in the high-frequency oscillation's amplitude; and another modulation in the amplitude of the local oscillations with period of the order of $30 \mathrm{~d}$. As discussed in Section 5.1, the short period of high-frequency local oscillations observed in Fig. 5 stems from the absence in our model equations of a convection parametrization. Comparing Figs. $4 \mathrm{~b}$ and $5 \mathrm{a}$ shows that the modulation with intermediate period (5-8 d) is a result of the energy exchanges among modes of the same triad. The same comparison indicates that the longest time-scale modulation, with period of the order of $30 \mathrm{~d}$, is associated with the energy exchanges between modes of different resonant triads (i.e. with the intertriad energy exchanges). Thus, Fig. 5 shows that the inclusion of two modes in the resonant three-wave problem implies in the increase of the vacillation cycles in the physical space solution, with the longest vacillation cycle being resultant from intertriad energy exchanges.

Figure $5 \mathrm{~b}$ indicates that with the amplitude of the highestfrequency mode multiplied by a factor 4 , the same local oscillations and changes of regime occur, but in a shorter period. It can be noted in Fig. $5 \mathrm{~b}$ that the longest cycle modulation of the local oscillations has a period of the order of $20 \mathrm{~d}$. As in Fig. 2, the unrealistic peak magnitude of the wind field observed in Fig. $5 b$ (of the order of $40 \mathrm{~m} \mathrm{~s}^{-1}$ ) is due to the somewhat unrealistic high initial wave amplitudes set in this numerical simulation $\left(A_{1}=4\right)$. Nevertheless, it is important to state again that this somewhat unrealistic high amplitude has been chosen in order to more clearly notice the dependence of the period of the longest period vacillation resultant from the intertriad interactions upon the initial amplitudes of the wave modes. 
Therefore, the numerical results of the problem of two resonant triads coupled by a single mode discussed in the present item of this section show that a significant interaction between modes of different triads is only possible if the mode coupling the two triads is a high-frequency mode (inertio-gravity or Kelvin mode). Especially, significant intertriad energy exchanges can occur if the mode that couples the triads is the highest-frequency mode of the two triads, as shown in the numerical results of this subsection. In this case, in addition to the interaction among wave components of the same resonant triad, effective energy exchanges involving modes of different triads take place in a longer time-scale than the intratriad ones. As the main energy source of the tropical atmospheric circulation is very scale selective (Kuo, 1975; Lau and Peng, 1987; Wang and Rui, 1990), the results suggest that the high-frequency modes, such as inertiogravity and Kelvin waves, are important in redistributing energy throughout the spectrum of the atmospheric motions and, in turn, in generating large-scale turbulence in the atmospheric flow. As shown in Figs. 4 and 5, for the initial conditions considered here we have obtained periods for the lowest-frequency modulation of the solution of problem (5.5), associated with the intertriad energy exchanges, between 10 and $30 \mathrm{~d}$, depending on the value of the initial energy and the way in which this initial energy is distributed among the wave components. Certainly, had we considered initial conditions with amplitudes smaller than those considered here, these low-frequency modulations associated with intertriad energy exchanges would have undergone periods longer than those observed here. Moreover, in a more realistic model containing more coupled resonant triads, based on the results presented here, there might be modulations with periods even longer. Thus, the results obtained here also suggest that the low-frequency variability of the atmospheric circulation, such as the intraseasonal and/or even longer period variability, can also be associated with non-linear energy exchanges involving modes of different resonant triads. In this process, as shown in the numerical results, the presence of the high-frequency modes coupling the resonant triads is crucial. Thus, the results also suggest the importance of inertio-gravity waves for the low-frequency variability (intraseasonal and/or even longer term) of the atmospheric flow.

\section{Summary and conclusions}

In the present work we have investigated the kinematical and dynamical properties of resonant interactions among equatorial waves in the context of the shallow-water model on the equatorial $\beta$-plane. The spectral method with the basis functions given by the eigensolutions of the linear problem was applied to the governing equations. In this context, from the phase space expansion of some quadratic to lowest-order integrals of motion of the model, some constraints were obtained which the coupling coefficients must satisfy in order to ensure the invariance of such integrals up to third order. From the numerical computa- tion of the coupling coefficients, these constraints were used to determine the possible resonant interactions among equatorial waves. In general, the resonant triads obtained involve all the equatorial wave types and are strongly dominated by triads with two high-frequency modes (Kelvin or inertio-gravity) and one low-frequency mode (Rossby or mixed Rossby-gravity). Some strictly low-frequency and strictly high-frequency triads also exist, but no triads involving solely the inertio-gravity modes could be found. In order to analyze the dynamics of these resonant interactions, the results of numerical integrations of the resonant three-wave problem and the problem of two resonant triads coupled by a single mode have also been discussed. In general, as a consequence of the conservation of the quadratic part of total energy and pseudo-momentum, the resonant triads involving equatorial waves form closed systems in which the mode energies evolve periodically in time, with the period and amplitude of the energy modulations being dependent on the initial condition, that is, dependent on the initial energy and the way in which this energy is distributed among the triad components. From the spectral reconstitution of the physical space solution, the time evolution of this solution shows that the periodic energy exchanges among the triad components imply in periodic changes of regime in the physical space solution. These changes of regime, in turn, occur in a longer time-scale than the period of the local oscillations associated with the phase propagation of the waves. This mechanism provides a possible source of intraseasonal variability in the model atmosphere.

As a consequence of the conservation of the quadratic part of total pseudo-momentum, the coupling coefficients in a resonant triad are proportional to the individual frequencies of the triad components. As a result, in the numerical integrations of both the resonant three-wave problem and the problem of two triads coupled by one mode the high-frequency modes were found to be energetically more active than the low-frequency modes. The latter tend to act as catalytic components in resonant interactions, with the exception occurring for the triads containing only strictly low-frequency modes. The numerical results of the problem of two triads coupled by one mode highlight the importance of the high-frequency modes for the redistribution of energy between different triads. As the main source of energy to the tropical atmosphere is latent heat release, which is very scale selective (Kuo, 1975; Lau and Peng, 1987; Wang and Rui, 1990), the results obtained here suggest the importance of the Kelvin and/or inertio-gravity waves for the redistribution of energy throughout the atmospheric motion spectrum and, consequently, for generating large-scale turbulence in the atmospheric flow.

As also shown by the numerical results of the problem of two resonant triads coupled by one mode, the intertriad energy exchanges provided by the high-frequency modes coupling the two triads occur in a longer time-scale than the intratriad interactions. Thus, the results also suggest that the low-frequency variability of the atmospheric flow, such as the intraseasonal and 
even longer period variability, can also be associated with vacillations resultant from the energy exchanges among modes of different resonant triads. In this process, as pointed out by the numerical results, the role of high-frequency modes is crucial, suggesting the importance of Kelvin and inertio-gravity waves (and therefore of the latent heat release) for the generation of the low-frequency variability of the tropical atmospheric circulation. As the inertio-gravity waves are directly associated with precipitation and moist convection, the results obtained in this paper clearly link the predictability and realistic representation of intraseasonal oscillations in atmospheric models on well founded moist convective parameterization. Dynamical forecast atmospheric models generally exhibit rather poor simulation and low predictability of the Madden-Julian Oscillation (MJO; Chen and Alpert, 1990; Lau and Chan, 1992; Hendon et al., 2000; Jones et al., 2000) and the results presented here propose that such a low skill may be due, at least in part, to the difficulty of the models in representing the interaction between moist convection and large-scale dynamics. An interesting analysis on the theoretical limit of predictability of the MJO by ensembles of twin predictability experiments has been performed by Waliser et al. (2003).

Therefore, considering the results of the problem of two resonant triads coupled by one mode presented in Section 5.2, this paper has also provided an alternative explanation for the origin of the MJO in the tropics. Since the discovery by Madden and Julian over two decades ago (Madden and Julian, 1972), the MJO has continued to be a topic of significant interest due to its clear modulation on the weather conditions and the wide range of phenomena with which it interacts. The main theoretical efforts to physically explain the origin of the MJO and the associated intraseasonal variability of the atmospheric circulation have been basically associated with either the dynamics of convectively coupled Kelvin-Rossby modes or the extratropical forcing mechanism. The former explains the MJO in terms of the linear equatorial Kelvin-Rossby wave dynamics modified by frictional damping and/or coupling with convection (Chang, 1977; Emanuel, 1987; Neelin et al., 1987; Lau and Peng, 1987; Chang and Lim, 1988; Wang, 1988; Wang and Rui, 1990; Moskowitz and Bretherton, 2000). The latter argues that the MJO can be triggered by extratropical Rossby-wave disturbances propagating intermittently toward the equator (Hsu et al., 1990; Kiladis and Weickmann, 1992; Matthews and Kiladis, 1998). The results presented here suggest a possible excitation mechanism in which the intraseasonal variability is associated with vacillations resultant from energy exchanges involving modes of different resonant triads of equatorial waves. In this nonlinear mechanism, as discussed previously, the role of the inertiogravity modes coupling different triads is essential. However, it is important to say that certain caution is necessary in posing the results of this paper as another possible explanation for the origin of the MJO and the associated intraseasonal variability of the tropical atmospheric circulation, because the period of the vacillations resultant from the energy modulations is rather sensitive to the initial wave amplitudes.

Finally, although the present paper displays the potential importance of non-linear mechanisms coupling large-scale equatorial waves in the context of the equatorial shallow-water equations, caution is necessary in interpreting these results for the fully vertically stratified atmosphere, because the Galerkin projection of the non-linear three-dimension primitive equations onto a particular vertical mode does not fall naturally into the non-linear shallow-water equations given by eqs. (2.1) and (2.2), as discussed in Section 2. Moreover, the model adopted in the present work does not consider important physical mechanisms present in the real atmosphere, such as the interaction between moist convection and large-scale dynamics, forcing and dissipation, along with the vertical density stratification. We plan a future study which analyzes the existence of resonant interactions involving the main modes of variability of the large-scale atmospheric circulation (Rossby, inertio-gravity, mixed Rossbygravity and Kelvin modes) in a baroclinic atmospheric model, i.e. associated with different vertical modes. However, the simplified model used here might capture some of the essential ingredients of the more complex atmospheric dynamics.

\section{Acknowledgments}

The work presented in this paper is part of the ongoing research of the $\mathrm{PhD}$ program of the first author developed at University of São Paulo. This PhD program has been financially supported by FAPESP (Fundação de Amparo à Pesquisa do Estado de São Paulo) under GRANT 02/09683-9. The authors also acknowledge the support by the program 'Instituto do Milênio-Avanço Global e Integrado da Matemática Brasileira’ (IM-AGIMB). The present work is also part of the research proposed by the PROSUR program, financially supported by the IAI. The authors are also grateful to Dra. Maria Assunção Faus da Silva Dias for the fruitful suggestions to the present paper.

\section{References}

Boville, B. 1980. Amplitude vacillation on an f-plane. J. Atmos. Sci. 37, 1413-1423.

Bretherton, F. P. 1964. Resonant interactions between waves: the case of discrete oscillations. J. Fluid. Mech. 20, 457-479.

Chang, C.-P. 1977. Viscous internal gravity waves and low-frequency oscillations in the tropics. J. Atmos. Sci. 34, 901-910.

Chang, C.-P. and Lim, H. 1988. Kelvin wave Cisk: a possible mechanism for the 30-50 day oscilations. J. Atmos. Sci. 45, 1709-1720.

Charney, J. G. 1971. Geostrophic turbulence. J. Atmos. Sci. 28, 10871095.

Chen, T.-C. and Alpert, J. C. 1990. Systematic errors in the annual and intraseasonal variations of the planetary-scale divergent circulation in NMC medium-range forecasts. Mon. Wea. Rev. 118, 2607-2623.

DeMaria, M. 1985. Linear response of a stratified tropical atmosphere to convective forcing. J. Atmos. Sci. 42(18), 1944-1959.

Domaracki, A. and Loesch, A. Z. 1977. Nonlinear interactions among equatorial waves. J. Atmos. Sci. 34, 486-498. 
Dunkerton, T. and Baldwin, M. 1995. Observation of 3-6 day meridional wind oscillations over the tropical Pacific, 1973-1992: horizontal structure and propagation. J. Atmos. Sci. 52(10), 15851601.

Emanuel, K. A. 1987. An air-sea interaction model of intraseasonal oscillations in the tropics. J. Atmos. Sci. 44, 2224-2240.

Gill, A. E. 1980. Some simple solutions for heat-induced tropical circulation. Q. J. R. Meteorol. Soc. 106, 447-462.

Gruber, A. 1974. The wavenumber-frequency spectra of satellite measured brightness in the tropics. J. Atmos. Sci. 31, 1675-1680.

Hendon, H. H., Liebmann, B., Newman, M., Glick, J. D. and Schemm, J. 2000. Medium-range forecast errors associated with active episodes of the Madden-Julian Oscillation. Mon. Wea. Rev. 128, 69-86.

Hirst, A. C. 1986. Unstable and damped equatorial modes in simple coupled ocean-atmosphere models. J. Atmos. Sci. 43, 606-630.

Hsu, H.-H., Hoskins, B. and Jin, F.-F. 1990. The 1985/86 intraseasonal oscillation and the role of the extratropics. J. Atmos. Sci. 47, 823839.

Jones, C., Waliser, D. E., Schemm, J. K. and Lau, W. K. 2000. Prediction skill of the Madden-Julian oscillation in dynamical extended range forecasts. Climate Dyn. 16, 273-289.

Kanamitsu, M., Krishnamurti, T. N. and Depradine, C. 1972. On scale interactions in the tropics during northern summer. J. Atmos. Sci., 29, 698-706.

Kiladis, G. N. and Weickmann, K. M. 1992. Extratropical forcing of tropical Pacific convection during northern winter. Mon. Wea. Rev. 120, 1924-1938.

Kuo, H. L. 1975. Instability theory of large-scale disturbances in the tropics. J. Atmos. Sci. 32, 2229-2245.

Lau, K.-M. 1981. Oscillation in a simple equatorial climate system. J. Atmos. Sci. 38, 248-261.

Lau, K.-M. and Chan, P. H. 1992. Tropical intraseasonal oscillation and its prediction by the NMC operational model. J. Climate. 5, 13651378.

Lau, K.-M. and Lim, H. 1982. Thermally driven motions in an equatorial $\beta$-plane: Hadley and Walker circulations during the winter monsoon. Mon. Wea. Rev. 110, 336-353.

Lau, K.-M. and Peng, L. 1987. Origin of low-frequency (intraseasonal) oscillations in the tropical atmosphere. Part I: basic theory. J. Atmos. Sci. 44, 950-972.

Lim, H. and Chang, C.-P. 1983. Dynamics of teleconnections and walker circulations forced by equatorial heating. J. Atmos. Sci. 40, 18971915.

Lindzen, R. S., Farrell, B. and Jacqmin, D. 1982. Vacillations due to wave interference: applications to the atmosphere and to annulus experiments. J. Atmos. Sci. 39, 14-23.

Loesch, A. Z. and Deininger, R. C. 1979. Dynamics of closed systems of resonantly interacting equatorial waves. J. Atmos. Sci. 36, 1490-1497.

Longuet-Higgins, M. S. and Gill, A. E. 1967. Resonant interactions between planetary waves. Proc. R. Soc. London A 299, 120.

Lorentz, E. N. 1963. The mechanics of vacillation. J. Atmos. Sci. 20, 448-464.

Madden, R. A. and Julian, P. 1972. Description of global-scale circulation cells in the tropics with a 40-50 day period. J. Atmos. Sci. 29, 11091123.

Magaña, V. and Yanai, M. 1995. Mixed Rossby-gravity waves triggered by lateral forcing. J. Atmos. Sci. 52(9), 1473-1486.
Majda, A. J., Rosales, R. R., Tabak, E. and Turner, C. V. 1999. Interaction of long-scale equatorial waves and dispersion of Kelvin waves through topographic resonances. J. Atmos. Sci. 56, 4118-4133.

Maruyama, T. 1967. Large-scale disturbances in the equatorial lower stratosphere. J. Meteorol. Soc. Japan 45, 391-408.

Matsuno, T. 1966. Quasi-geostrophic motions in the equatorial area. J. Meteorol. Soc. Japan 44, 25-43.

Matthews, A. J. and Kiladis, G. N. 1998. The tropical-extratropical interaction between high-frequency transients and the Madden-Julian oscillation. Mon. Wea. Rev. 127, 661-677.

Moskowitz, B. M. and Bretherton, C. S. 2000. An analysis of frictional feedback on a moist equatorial Kelvin mode. J. Atmos. Sci. 57, 2188 2206.

Neelin, J. D., Held, I. M. and Cook, K. H. 1987. Evaporation-wind feedback and low-frequency variability in the tropical atmosphere. J. Atmos. Sci. 44, 2341-2348.

Ott, E. 1993. Chaos in Dynamical Systems. Cambridge University Press, Cambridge, 385 pp.

Pedlosky, J. 1977. A model of wave amplitude vacillation. J. Atmos. Sci. 34, 1898-1912.

Phillips, O. M. 1960. On the dynamics of unsteady gravity waves of finite amplitude. J. Fluid Mech. 9, 193-217.

Pires, P., Redelsperger, J. L. and Lafore, J. P. 1997. Equatorial atmospheric waves and their association to convection. Mon. Wea. Rev. 6(14), 1167-1184.

Raupp, C. F. M. and Silva Dias, P. L. 2004. Effects of nonlinear process on the interhemispheric energy propagation forced by tropical heat sources. Brazilian J. Meteorol. 19(2), 177-188.

Raupp, C. F. M. and Silva Dias, P. L. 2005. Excitation mechanism of mixed Rossby-gravity waves in the equatorial atmosphere: role of the nonlinear interactions among equatorial waves. J. Atmos. Sci. 62, 1446-1462.

Ripa, P. 1981. On the theory of nonlinear interactions among geophysical waves. J. Fluid Mech. 103, 87-115.

Ripa, P. 1982. Nonlinear wave-wave interactions in a one-layer reducedgravity model on the equatorial $\beta$-plane. J. Phys. Oceanogr. 12, 97111.

Ripa, P. 1983a. Weak interactions of equatorial waves in a one-layer model. Part I: general properties. J. Phys. Oceanogr. 13, 12081226.

Ripa, P. 1983b. Weak interactions of equatorial waves in a one-layer model. Part II: applications. J. Phys. Oceanogr. 13, 1227-1240.

Salmon, R. 1980. Geostrophic turbulence. Proceedings of the Enrico Fermi International School of Physics, Varenna, Italy.

Silva Dias, P. L. and Bonatti, J. P. 1985. A preliminary study of the observed modal structure of the Summer circulation over tropical South America. Tellus 37A, 185-195.

Silva Dias, P. L. and Schubert, W. H. 1979. The dynamics of equatorial mass-flow adjustment. Atmospheric Science Paper No. 312, Department of Atmospheric Science Colorado State University, Fort Collins, CO, USA.

Silva Dias, P. L., Schubert, W. H. and DeMaria, M. 1983. Large-scale response of the tropical atmosphere to transient convection. J. Atmos. Sci. 40, 2689-2707.

Takayabu, Y. 1994. Large-scale cloud disturbance associated with equatorial waves. Part I: spectral features of the cloud disturbances. $J$. Meteorol. Soc. Japan 72, 433-448. 
Waliser, D. E., Lau, K. M., Stern, W. and Jones, C. 2003. Potential predictability of the Madden-Julian oscillation. Bull. Am. Meteorol. Soc. 84(1), 33-50.

Wang, B. 1988. Dynamics of tropical low-frequency waves: an temperatureanalysis of the moist Kelvin wave. J. Atmos. Sci. 45, 2051-2065.

Wang, B. and Rui, H. 1990. Dynamics of the coupled moist KelvinRossby wave on an equatorial $\beta$-plane. J. Atmos. Sci. 47, 397-413.

Webster, P. J. 1972. Response of the tropical atmosphere to local steady forcing. Mon. Wea. Rev. 100, 518-540.
Wheeler, M. and Kiladis, G. N. 1999. Convectively coupled equatorial waves: Analysis of clouds and temperature in the wavenumberfrequency domain. J. Atmos. Sci. 56, 374-399.

Yanai, M. and Maruyama, T. 1966. Stratospheric wave disturbances propagating over the equatorial Pacific. J. Meteorol. Soc. Japan 44, 291294.

Zangvil, A. and Yanai, M. 1980. Upper tropospheric waves in the tropics. Part 1: dynamical analysis in the wavenumber-frequency domain. J. Atmos. Sci. 37, 283-298. 